\title{
Data assimilation of CrIS and TROPOMI satellite CO concentrations and its potential for constraining global $\mathrm{OH}$
}

by

Nadine Terumi Maria de Bruyn

\begin{abstract}
A thesis submitted to the Faculty of Graduate and Postdoctoral Affairs in partial fulfillment of the requirements for the degree of
\end{abstract}

\author{
Master of Applied Science \\ in \\ Environmental Engineering \\ Carleton University \\ Ottawa, Ontario
}

(C) 2021, Nadine Terumi Maria de Bruyn 


\section{Abstract}

This work investigates the possibility of using high resolution carbon monoxide (CO) observations to constrain global mean hydroxyl radical concentrations $\left([\mathrm{OH}]_{\mathrm{GM}}\right)$. An improved observational bound on $[\mathrm{OH}]_{\mathrm{GM}}$ would enhance understanding of chemical reaction rates in the atmosphere, specifically the lifetime of greenhouse gases like methane, and improve global atmospheric model calculations.

A multispectral retrieval combining radiances from the Cross-Track Infrared Sounder (CrIS) and TROPOspheric Monitoring Instrument (TROPOMI) satellite instruments has potential to provide high vertical resolution of tropospheric $\mathrm{CO}$. These observations are evaluated against existing measurements from Network for the Detection of Atmospheric Composition Change (NDACC) stations, the Measurement of Pollution in the Troposphere (MOPITT) satellite, and GEOS-Chem chemical transport model calculations. GEOS-Chem adjoint is used to determine sensitivities of each observing platform to estimated GEOS-Chem $\mathrm{OH}$ abundance in the troposphere. This should provide insight into $\mathrm{OH}$ reaction rates with $\mathrm{CO}$, and whether $\mathrm{CO}$ could be used to estimate $[\mathrm{OH}]_{G M}$. 


\section{Acknowledgements}

Over the course of my studies at Carleton University I received significant support from numerous persons in my academic, professional, and personal life. With their help I was able to expand my curiosity while persevering through a pandemic towards the end of my studies. It was a great pleasure to conduct research under my thesis supervisor Dr. Thomas Walker. His extensive knowledge, insight, guidance, patience, support, and positivity was and continues to inspire me to strive for the best in my work. I am extremely grateful to have him as my graduate supervisor, as would any future students.

I would also like to extend my gratitude to those who were instrumental in contributing data and technical support allowing for this research to be possible. I am grateful for Carleton University's Research Computing Specialist (RCS) Ryan Taylor's assistance. He led courses on the use of Carleton's Linux server, fixed model compatibilities, and swiftly aided me to resolve technical issues. Special thanks to Nadia Smith and Christopher D. Barnet from Science and Technology Corp. (STC) for providing essential assistance with NOAA/NASA Joint Polar Satellite System (JPSS) CLIMCAPS data product and processing. Thanks to Helen M. Worden and Dejian Fu for their CrIS and TROPOMI satellite resolution examinations, opening the floor to new investigations including exploring use for determining global $\mathrm{OH}$. I would also like to thank Merrit N. Deeter, James R. Drummond, and Helen M. Worden again for continuous MOPITT research, providing a base comparison for this research.

Finally, I am grateful for my friends and family for their continuous support and motivation throughout my studies. I extend this gratitude to Dr. Mark Gibson, an inspirational 
Professor from my undergraduate studies at Dalhousie University who first sparked my interest in the air quality field, and to Jay Stukel of Niagara Counselling \& Wellness who gave me the courage to pursue my academic and professional dreams. 


\section{Table of Contents}

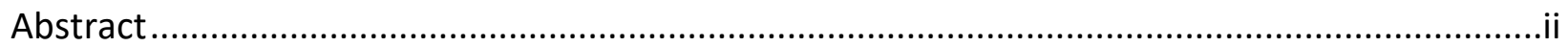

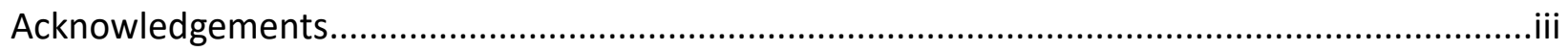

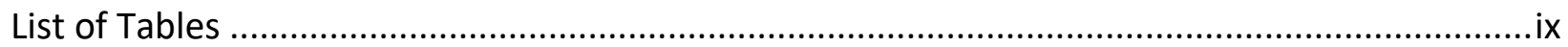

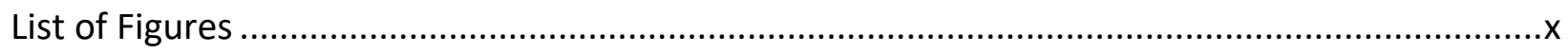

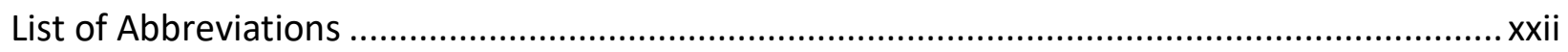

List of Chemical Formulas ......................................................................................................

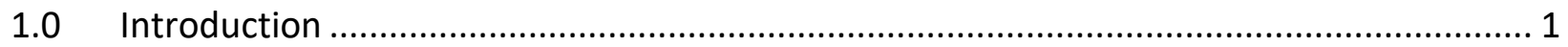

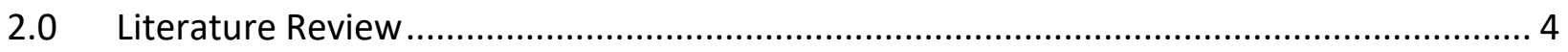

$2.1 \quad$ Earth's Atmosphere................................................................................................ 5

2.1.1 Atmospheric structure and influences ……......................................................... 5

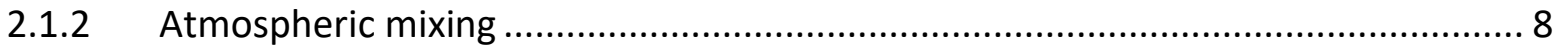

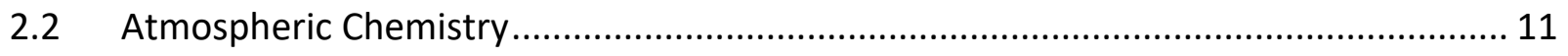

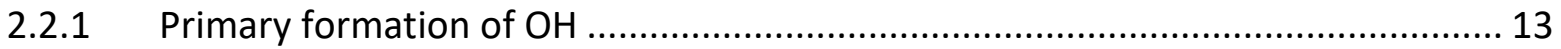

2.2.2 $\mathrm{OH}$ depletion, secondary formation, recycling, and termination......................... 15

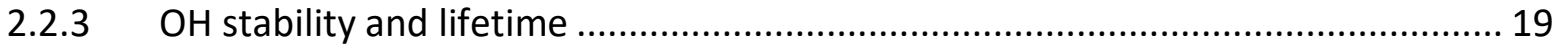

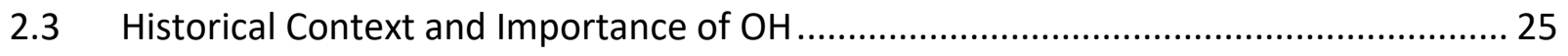

2.3.1 History of measuring and calculating $\mathrm{OH}$.......................................................... 25

2.3.2 Historical Tropospheric Oxidative Capacity ............................................................ 35 
2.4 Remote sensing, radiation, and the atmosphere

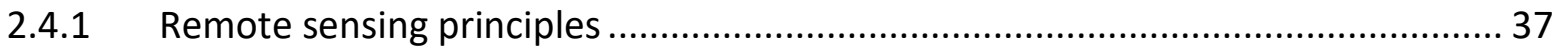

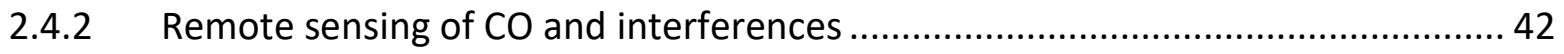

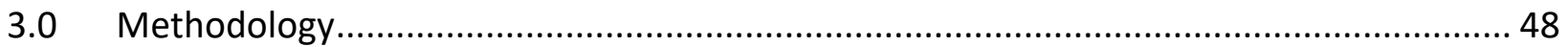

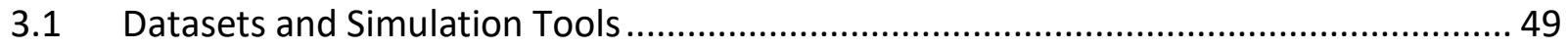

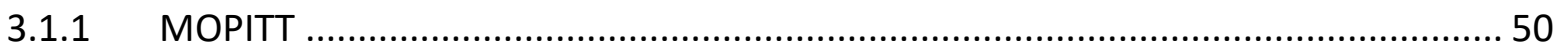

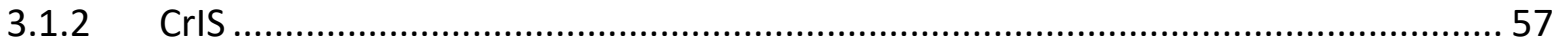

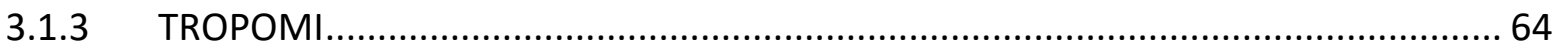

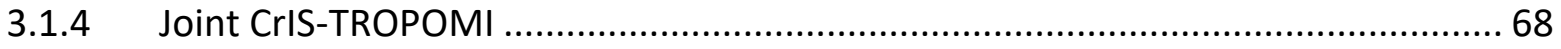

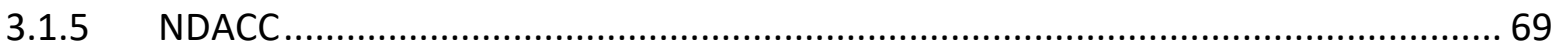

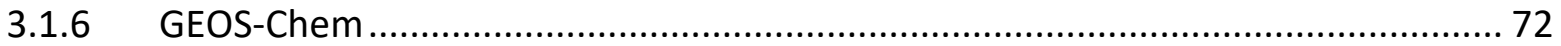

3.1.7 GEOS-Chem Adjoint ............................................................................ 75

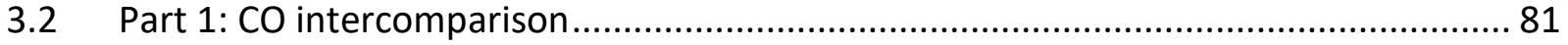

3.2.1 CO retrieval and calculations in preparation for intercomparison....................... 82

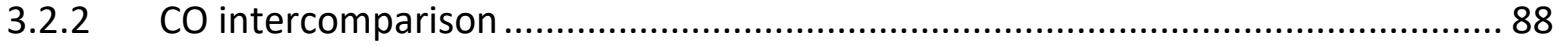

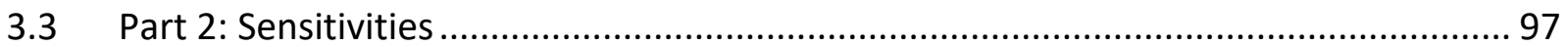

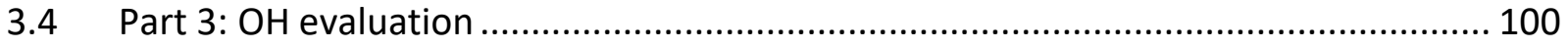

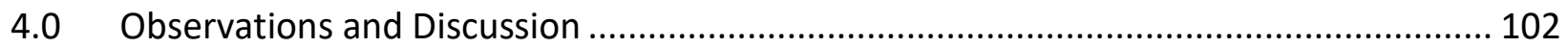

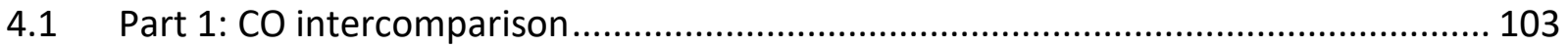


4.1.1 Total column comparison observations

4.1.2 Profile comparison observations ............................................................ 113

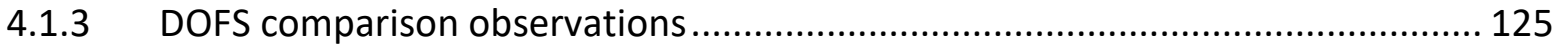

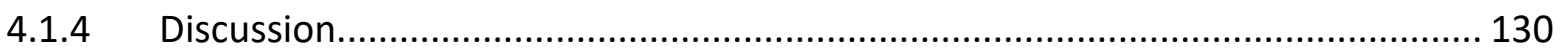

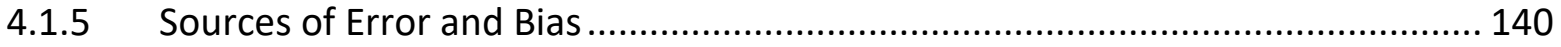

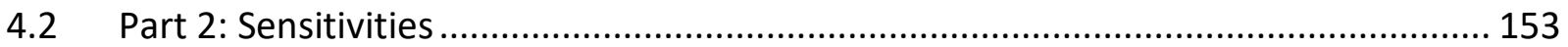

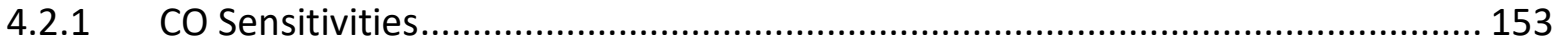

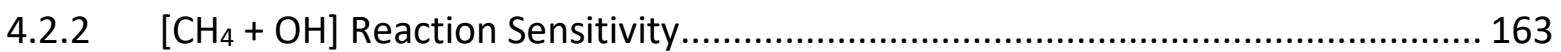

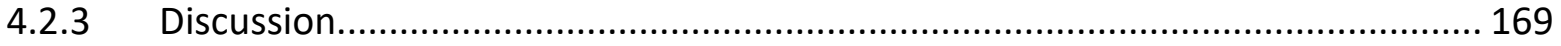

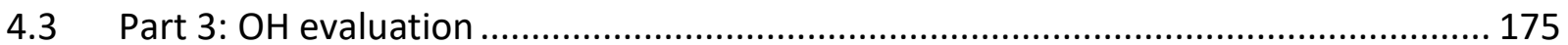

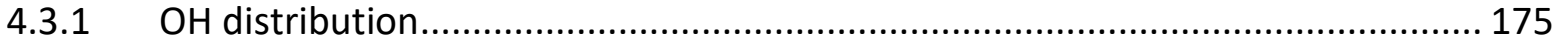

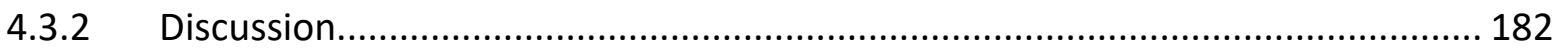

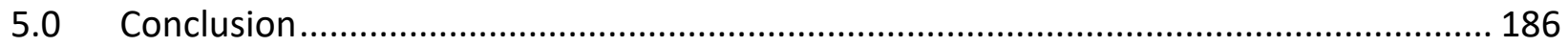

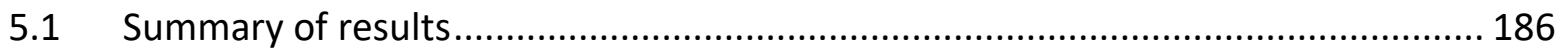

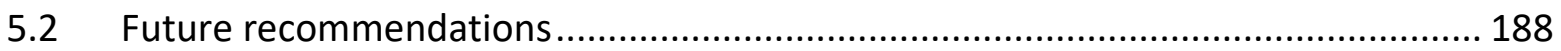

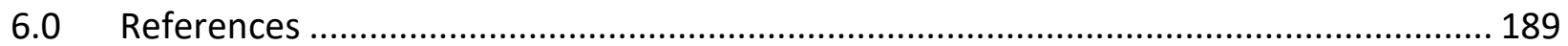

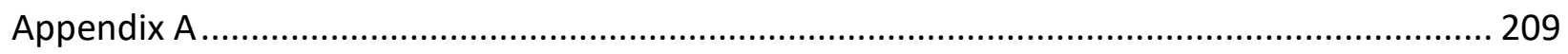

A.1 Table of NDACC station locations and information ................................................ 209

A.2 MOPITT CO retrieval algorithm flow chart of processing ................................... 212 
A.3 CrIS NUCAPS CO retrieval algorithm flow chart ................................................. 213

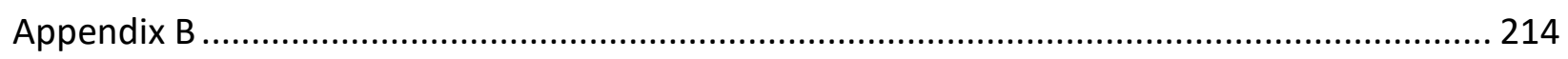

B.1 Cross-kernel RMSE comparisons for high altitude NDACC stations ........................ 214

B.2 Additional cross-kernel monthly mean RMSE profile sections ............................ 217

B.3 Additional profile error section graphs ........................................................ 221

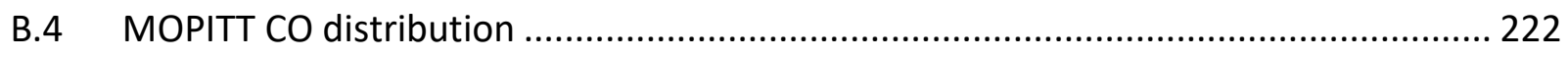

B.5 CO emission sensitivity differences............................................................ 223

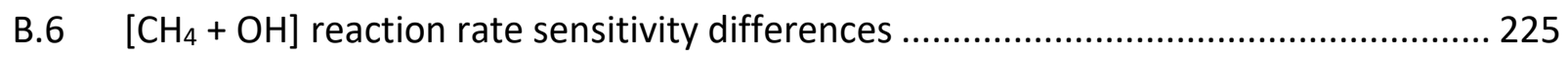

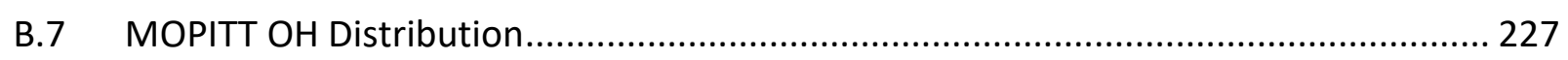




\section{List of Tables}

Table 2.3.1.1: Summary of select global $\mathrm{OH}$ abundance, interannual variations and interhemispheric values determined in previous journal articles and reports 33

Table 3.2.1.1: Summary of prepared cross-kernels for intercomparison. " $P$ " and " $T$ " indicate where profile and total column cross-kernels could be calculated, respectively. 88

Table 3.2.2.1: Summary of observing platforms' units and atmospheric state information

provided in their data products.

Table 4.1.2.1: Total average four-month mean profile RMSE (ppm) of all stations across all cross-kernel calculations between instruments. Note that the NDACC cross-kernel average values include a column where all station values are averaged, and a column where the outliers are removed from the average.

Table 4.3.1.1: Global mean $\mathrm{OH}\left(\right.$ molecules $/ \mathrm{cm}^{3}$ ) and mean $\mathrm{NH} / \mathrm{SH}$ ratio for the GEOS-Chem adjoint base model output for each month, May through August 2016, below 100 hPa. Each global mean $\mathrm{OH}$ and $\mathrm{NH} / \mathrm{SH}$ ratio are provided in airmass, $\mathrm{CO}$, and volume weighted values. 177 Table 5.2.1: Table of NDACC station locations and information used in this study. 209 Table 5.2.1: MOPITT CO retrieval algorithm flow chart for Level 0 to Level 3 data (Francis et al., 2017)

Table A.3.1: CrIS NUCAPS CO retrieval algorithm for Level 1 to Level 2 data (Gambacorta, 2013) 


\section{List of Figures}

Figure 2.1.1.1: Mean pressure and temperature vs. altitude at $30^{\circ} \mathrm{N}$, March (Jacob, 2000)........ 6

Figure 2.1.2.1: Diagram of atmospheric circulation depicting the three cells of the atmosphere, resulting trade winds, and the intertropical convergence zone (ITCZ). (Encyclopedia Britannica,

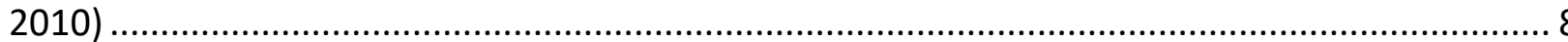

Figure 2.1.2.2: Vertical (left) and horizontal (right) atmospheric mixing times. (Jacob, 2000).... 10

Figure 2.1.2.3: Seasonal ITCZ shift and lateral mixing between high and low pressure zones.

(Lutgens \& Tarbuck, 2001)

Figure 2.2.1.1: Global spatial distribution of mean $\mathrm{OH}$ concentrations depicted by the GEOSChem base model for May 2016. The top plot shows the mean zonal vertical distribution in molecules $/ \mathrm{cm}^{3}$ through the modeled atmosphere. The bottom plot shows horizonal $\mathrm{OH}$ total column distribution in molecules $/ \mathrm{cm}^{2}$, the white contour depicting the approximate $80^{\text {th }}$

percentile.

Figure 2.4.1.1: Liou (2002) solar irradiance curve depicting absorption and scattering by difference atmospheric species.

Figure 2.4.1.2: Solar reflection and terrestrial emission in comparison to the observed MOPITT instrument $\mathrm{CO}$ absorption spectra. Figure retrieved from the MOPITT Algorithm Theoretical Basis Document (ATBD), August 1996

Figure 2.4.2.1: NIST Chemistry WebBook carbon monoxide first-overtone band.

Figure 3.1.2.1: CrIS CLIMCAPS CO retrieval algorithm flow chart of processing Level 1 data to Level 2 data (Smith \& Barnet, 2019) 
Figure 3.1.3.1: TROPOMI CO retrieval algorithm flow chart of processing Level 1 data to Level 2 data (Landgraf et al., 2018) 66

Figure 3.1.7.1: Effect of the regularisation parameter $\gamma \mathrm{r}$ (shown here as global weighting factor $\mu$ ) on model prediction and assimilation error as demonstrated in Hakami et. al. (2005). At $\mu=$ 1 , the model prediction error is equivalent to the initial model model error, and the assimilation error is equivalent to the observation error before assimilation. 81

Figure 3.2.1.1: A unique Toronto NDACC station CO averaging kernel for May $3^{\text {rd }}$ 2016, where each matrix row is plotted as a NDACC pressure layer represented in the legend.

Figure 3.2.1.2: (Left) Smoothed CO profile retrievals of each instrument over Toronto for May $3^{\text {rd }}$ with no cross-kernels applied. (Right) Cross-kernel smoothed profiles for NDACC over Toronto for May $3^{\text {rd }}$. The NDACC smoothed profile and error are shown in orange. The NDACC a priori and averaging kernel are applied to the smoothed retrievals of the other observing platforms. 85

Figure 3.2.2.1: Global map of all NDACC station locations utilised in this study (red dots), and their $200 \mathrm{~km}$ radius (blue). 91 Figure 3.2.2.2: Map of select NDACC stations utilised in the study over Europe (red dots), their $200 \mathrm{~km}$ radius (blue), and the GEOS-Chem rectilinear $2^{\circ} \times 2.5^{\circ}$ grid. Note the difference in number of grid cells within the $200 \mathrm{~km}$ radius of each station. 91

Figure 4.1.1.1: Four-month mean total column percent difference of each NDACC station location. (Left) Total column \% difference between observing platforms and NDACC stations when no cross-kernel calculation is applied. (Middle) Total column \% difference between observing platforms and NDACC total columns when the NDACC cross-kernel calculation is 
applied, except not applied to TROPOMI. (Right) Total column \% difference between observing platforms and MOPITT total columns when the MOPITT cross-kernel calculation is applied, except not applied to TROPOMI. 105

Figure 4.1.1.2: NDACC cross-kernel monthly mean total column percent difference trends against each observing platform. i) CrIS total column percent difference for NDACC cross-kernel. ii) MOPITT total column percent difference for NDACC cross-kernel. iii) GEOS-Chem total column percent difference for NDACC cross-kernel. iv) TROPOMI total column percent difference against NDACC with no cross-kernel calculation applied. 106

Figure 4.1.1.3: MOPITT cross-kernel monthly mean total column percent difference trends against each observing platform. i) CrIS total column percent difference for MOPITT crosskernel. ii) GEOS-Chem total column percent difference for MOPITT cross-kernel. iii) NDACC total column percent difference for MOPITT cross-kernel. iv) TROPOMI total column percent difference against MOPITT with no cross-kernel calculation applied. 107

Figure 4.1.2.1: Four-month mean CO profile RMSE (ppm) for NDACC Cross-Kernel comparisons. Full profile and section profile comparisons are shown. Order from left to right are the RMSE comparisons for the full profile, $\sim 100 \mathrm{hPa}$ to the surface, $\sim 100 \mathrm{hPa}$ to the approximate top of the boundary layer (BL) at $\sim 800 \mathrm{hPa}$, and the values within the $\mathrm{BL}$ to the surface. 114 Figure 4.1.2.2: Four-month mean CO profile RMSE (ppm) for MOPITT Cross-Kernel comparisons. Full profile and section profile comparisons are shown. Order from left to right are the RMSE comparisons for the full profile, $\sim 100 \mathrm{hPa}$ to the approximate top of the boundary layer (BL) at $\sim 800 \mathrm{hPa}$, and the values within the BL to the surface. Note that the MOPITT full profile is the same as $\sim 100 \mathrm{hPa}$ to the surface. 115 
Figure 4.1.2.3: Four-month mean CO profile RMSE (ppm) for CrIS Cross-Kernel comparisons. Full profile and section profile comparisons are shown. Order from left to right are the RMSE comparisons for the full profile, $\sim 100 \mathrm{hPa}$ to the surface, $\sim 100 \mathrm{hPa}$ to the approximate top of the boundary layer $(\mathrm{BL})$ at $~ 800 \mathrm{hPa}$, and the values within the $\mathrm{BL}$ to the surface. 116 Figure 4.1.2.4: NDACC cross-kernel monthly mean profile RMSE (ppm) trends against each observing platform from $100 \mathrm{hPa}$ to the approximate top of the boundary layer (BL). i) CrIS profile RMSE for NDACC cross-kernel from $100 \mathrm{hPa}$ to the BL. ii) MOPITT profile RMSE for NDACC cross-kernel from $100 \mathrm{hPa}$ to the BL. iii) GEOS-Chem profile RMSE for NDACC crosskernel from $100 \mathrm{hPa}$ to the BL. Stations bolded black in the legend have values off the chart. Stations bolded grey in the legend have no values over the full four months.

Figure 4.1.2.5: MOPITT cross-kernel monthly mean profile RMSE (ppm) trends against each observing platform from $100 \mathrm{hPa}$ to the approximate top of the boundary layer (BL). i) CrIS profile RMSE for MOPITT cross-kernel from $100 \mathrm{hPa}$ to the BL. ii) GEOS-Chem profile RMSE for MOPITT cross-kernel from $100 \mathrm{hPa}$ to the BL. iii) NDACC profile RMSE for MOPITT cross-kernel from $100 \mathrm{hPa}$ to the BL. Stations bolded black in the legend have values off the chart. Stations bolded grey in the legend have no values over the four months. 121

Figure 4.1.2.6: CrIS cross-kernel monthly mean profile RMSE (ppm) trends against each observing platform from $100 \mathrm{hPa}$ to the approximate top of the boundary layer (BL). i) MOPITT profile RMSE for CrIS cross-kernel from $100 \mathrm{hPa}$ to the BL. ii) GEOS-Chem profile RMSE for CrIS cross-kernel from $100 \mathrm{hPa}$ to the BL. iii) NDACC profile RMSE for CrIS cross-kernel from $100 \mathrm{hPa}$ to the BL. Stations bolded black in the legend have values off the chart. Stations bolded grey in the legend have no values over the four months. 
Figure 4.1.3.1: Daily profile DOFS of each observing platform, averaged over all stations. Note that the slopes for NDACC and TROPOMI are not statistically significant when compared to their slope error. 128

Figure 4.1.3.2: Distribution of average daily profile DOFS values for each observing platform over each NDACC station.

Figure 4.1.4.1: CO profile CrIS cross-kernel comparisons for Eureka on May $14^{\text {th }}$, Bremen on June $23^{\text {rd }}$, and Toronto on July $20^{\text {th }}$. Note the profile deviation of every observing platform's CO concentration within the boundary layer. 135 Figure 4.1.4.2: CO profile CrIS cross-kernel comparisons for Lauder on May $11^{\text {th }}$, Reunion Island on May $30^{\text {th }}$, and Wollongong on May $30^{\text {th }}$. The CrIS cross-kernel calculation weights the other observing platforms' upper atmosphere concentrations to zero. 136 Figure 4.1.4.3: Profile cross-kernel comparisons between each observing platform over Ny Alesund on 2 August. Note that the CrIS observation is for the year 2019, and all other observations are for 2016. Across all cross-kernel comparisons, a characteristic bulge is seen for the NDACC and MOPITT observations, possibly indicating a 2016 anomaly event.

Figure 4.1.4.4: CO profile comparison with the NDACC cross-kernel calculation for Lauder on June $2^{\text {nd }}$, July $1^{\text {st }}$, and Aug $10^{\text {th }}$ (left to right). 138 Figure 4.1.4.5: CO profile comparison with no cross-kernel calculations applied for Lauder on June $2^{\text {nd }}$, July $1^{\text {st }}$, and Aug $10^{\text {th }}$ (left to right). 138

Figure 4.1.5.1: Daily average CO total column error average for each observing platform for May through August 2016. The total column error is the provided dataset error values plus the variability of the averaged points. 145 
Figure 4.1.5.2: Distribution of daily average CO total column error per station for May through August 2016. The total column error is the provided dataset error values plus the variability of the averaged points. 146

Figure 4.1.5.3: Daily average CO profile error from $100 \mathrm{hPa}$ to $\mathrm{BL}$ for each observing platform for May through August 2016. The profile errors are the provided dataset error values plus the variability of the averaged points.

Figure 4.1.5.4: Daily average CO profile error within the BL for each observing platform for May through August 2016. The profile errors are the provided dataset error values plus the variability of the averaged points. 147

Figure 4.1.5.5: Distribution of daily average CO profile error per station from the surface to 100 hPa for May through August 2016. The profile errors are the provided dataset error values plus the variability of the averaged points. 148

Figure 4.1.5.6: Distribution of daily average CO profile error per station from $100 \mathrm{hPa}$ to $\mathrm{BL}$ for May through August 2016. The profile errors are the provided dataset error values plus the variability of the averaged points.

Figure 4.1.5.7: Distribution of daily average CO profile error per station within the BL for May through August 2016. The profile errors are the provided dataset error values plus the variability of the averaged points. 150 Figure 4.2.1.1: GEOS-Chem base modeled mean global CO distribution for each month, May through August 2016. Figures on the left represent the CO zonal mean ppm across the globe. Figures on the right represent to total column CO concentration in molecules $/ \mathrm{cm}^{2}$. The white 
contour line represents the MOPITT integrated model global CO concentration's $80^{\text {th }}$ percentile.

Figure 4.2.1.2: MOPITT minus the GEOS-Chem base mean global CO distribution and the for each month, May through August 2016. Figures on the left represent the CO zonal mean ppm difference across the globe. Figures on the right represent to total column CO concentration difference in molecules $/ \mathrm{cm}^{2}$. Note that the MOPITT CO distribution used is the model output after one adjoint iteration.

Figure 4.2.1.3: GEOS-Chem mean base surface anthropogenic CO sensitivity [unitless] for each month, May through August 2016. 159

Figure 4.2.1.4: GEOS-Chem mean base surface biomass burning CO sensitivity [unitless] for each month, May through August 2016. 159

Figure 4.2.1.5: GEOS-Chem mean base surface biofuel CO sensitivity [unitless] for each month, May through August 2016. 160

Figure 4.2.1.6: MOPITT model mean surface anthropogenic CO sensitivity [unitless] for each month, May through August 2016. 161

Figure 4.2.1.7: MOPITT model mean surface biomass burning CO sensitivity [unitless] for each month, May through August 2016. 161

Figure 4.2.1.8: MOPITT model mean surface biofuel CO sensitivity [unitless] for each month, May through August 2016. 162

Figure 4.2.2.1: NDACC mean global $\left[\mathrm{CH}_{4}+\mathrm{OH}\right]$ reaction sensitivity [unitless] for each month, May through August 2016. 165 
Figure 4.2.2.2: MOPITT mean global $\left[\mathrm{CH}_{4}+\mathrm{OH}\right]$ reaction sensitivity [unitless] for each month,

May through August 2016. 167

Figure 4.3.1.1: GEOS-Chem base modeled mean global OH for each month, May through August 2016. The left-hand plot shows the GEOS-Chem base model's global, NH and SH mean OH in molecules $/ \mathrm{cm}^{3}$. The right-hand plot shows the difference between the GEOS-Chem base and MOPITT assimilation mean $\mathrm{OH}$ difference in molecules $/ \mathrm{cm}^{3}$. 177

Figure 4.3.1.2: Mean NH/SH OH ratio each month, May through August 2016. Blue representing the GEOS-Chem base model ratio, and orange representing the ratio difference between the GEOS-Chem and MOPITT assimilation model.

Figure 4.3.1.3: GEOS-Chem base modeled mean global OH distribution for each month, May through August 2016. Figures on the left represent the $\mathrm{OH}$ longitudinal molecules $/ \mathrm{cm}^{3}$ summation around the globe. Figures on the right represent to total column CO concentration in molecules $/ \mathrm{cm}^{2}$. The white contour line represents the MOPITT integrated model global OH concentration's $80^{\text {th }}$ percentile. 179 Figure 4.3.1.4: MOPITT minus the GEOS-Chem base global $\mathrm{OH}$ distribution and for each month, May through August 2016. Figures on the left represent the $\mathrm{OH}$ longitudinal molecules $/ \mathrm{cm}^{3}$ summation difference around the globe. Figures on the right represent to total column $\mathrm{OH}$ concentration difference in molecules $/ \mathrm{cm}^{2}$. Note that the MOPITT OH distribution used is the model output after one adjoint iteration. 181

Figure B.1.1: Profile CO concentrations of observing platforms over the NDACC station Altzomoni for July $25^{\text {th }}$ 214 
Figure B.1.2: Profile NDACC cross-kernel CO concentrations of observing platforms over the NDACC station Altzomoni for July $25^{\text {th }}$

Figure B.1.3: Profile MOPITT cross-kernel CO concentrations of observing platforms over the NDACC station Altzomoni for July $25^{\text {th }}$ 216

Figure B.1.4: Profile CrIS cross-kernel CO concentrations of observing platforms over the NDACC station Altzomoni for July 25th. 216

Figure B.2.1: NDACC cross-kernel monthly mean full profile RMSE (ppm) trends against each observing platform. i) CrIS full profile RMSE for NDACC cross-kernel. ii) MOPITT full profile RMSE for NDACC cross-kernel. iii) GEOS-Chem full profile RMSE for NDACC cross-kernel. Stations bolded black in the legend have values off the chart. Stations bolded grey in the legend have no values over the four months..... 217

Figure B.2.2: NDACC cross-kernel monthly mean profile RMSE (ppm) trends against each observing platform from $100 \mathrm{hPa}$ to the surface. i) CrIS profile RMSE for NDACC cross-kernel from $100 \mathrm{hPa}$ to the surface. ii) MOPITT profile RMSE for NDACC cross-kernel from $100 \mathrm{hPa}$ to the surface. iii) GEOS-Chem profile RMSE for NDACC cross-kernel from $100 \mathrm{hPa}$ to the surface. Stations bolded black in the legend have values off the chart. Stations bolded grey in the legend have no values over the four months. 217

Figure B.2.3: NDACC cross-kernel monthly mean profile RMSE (ppm) trends against each observing platform within the approximate boundary layer (BL) to the surface. i) CrIS profile RMSE for NDACC cross-kernel within the BL to the surface. ii) MOPITT profile RMSE for NDACC cross-kernel within the BL to the surface. iii) GEOS-Chem profile RMSE for NDACC cross-kernel 
within the BL to the surface. Stations bolded black in the legend have values off the chart. Stations bolded grey in the legend have no values over the four months.

Figure B.2.4: MOPITT cross-kernel monthly mean full profile RMSE (ppm) trends against each observing platform. i) CrIS full profile RMSE for MOPITT cross-kernel. ii) GEOS-Chem full profile RMSE for MOPITT cross-kernel. iii) NDACC full profile RMSE for MOPITT cross-kernel. Note that for MOPITT profiles, the full profile is $100 \mathrm{hPa}$ to the surface. Stations bolded black in the legend have values off the chart. Stations bolded grey in the legend have no values over the four months.

Figure B.2.5: MOPITT cross-kernel monthly mean profile RMSE (ppm) trends against each observing platform within the approximate boundary layer $(\mathrm{BL})$ to the surface. i) CrIS profile RMSE for MOPITT cross-kernel within the BL to the surface. ii) GEOS-Chem profile RMSE for MOPITT cross-kernel within the BL to the surface. iii) NDACC profile RMSE for MOPITT crosskernel within the BL to the surface. Stations bolded black in the legend have values off the chart. Stations bolded grey in the legend have no values over the four months.

Figure B.2.6: CrIS cross-kernel monthly mean full profile RMSE (ppm) trends against each observing platform. i) MOPITT full profile RMSE for CrIS cross-kernel. ii) GEOS-Chem full profile RMSE for CrIS cross-kernel. iii) NDACC full profile RMSE for CrIS cross-kernel. Stations bolded black in the legend have values off the chart. Stations bolded grey in the legend have no values over the four months. 219

Figure B.2.7: CrIS cross-kernel monthly mean profile RMSE (ppm) trends against each observing platform from $100 \mathrm{hPa}$ to the surface. i) MOPITT profile RMSE for CrIS cross-kernel from 100 hPa to the surface. ii) GEOS-Chem profile RMSE for CrIS cross-kernel from $100 \mathrm{hPa}$ to the 
surface. iii) NDACC profile RMSE for CrIS cross-kernel from $100 \mathrm{hPa}$ to the surface. Stations bolded black in the legend have values off the chart. Stations bolded grey in the legend have no values over the four months. 220

Figure B.2.8: CrIS cross-kernel monthly mean profile RMSE (ppm) trends against each observing platform within the approximate boundary layer $(\mathrm{BL})$ to the surface. i) MOPITT profile RMSE for CrIS cross-kernel within the BL to the surface. ii) GEOS-Chem profile RMSE for CrIS cross-kernel within the BL to the surface. iii) NDACC profile RMSE for CrIS cross-kernel within the BL to the surface. Stations bolded black in the legend have values off the chart. Stations bolded grey in the legend have no values over the four months. 220

Figure B.2.1: Daily average CO full profile error average for each observing platform for May through August 2016. The profile errors are the provided dataset error values plus the variability of the averaged points.

Figure B.2.2: Daily average CO profile error average from the surface to $100 \mathrm{hPa}$ for each observing platform for May through August 2016. The profile errors are the provided dataset error values plus the variability of the averaged points

Figure B.3.1: MOPITT modeled mean global CO distribution for each month, May through August 2016. Figures on the left represent the CO zonal mean ppm across the globe. Figures on the right represent to total column CO concentration in molecules $/ \mathrm{cm}^{2}$. Note that the MOPITT CO distribution used is the model output after one adjoint iteration. The white contour line represents the MOPITT integrated model global CO concentration's $80^{\text {th }}$ percentile. 222 Figure B.4.1: MOPITT model minus GEOS-Chem base surface anthropogenic CO sensitivity [unitless] for each month, May through August 2016. 
Figure B.4.2: MOPITT model minus GEOS-Chem base surface biomass burning CO sensitivity [unitless] for each month, May through August 2016.

Figure B.4.3: MOPITT model minus GEOS-Chem base surface biofuel CO sensitivity [unitless] for each month, May through August 2016.

Figure B.5.1: MOPITT minus NDACC global $\left[\mathrm{CH}_{4}+\mathrm{OH}\right]$ reaction sensitivity [unitless] for each month, May through August 2016. Note that the figure scales on the left is different in May than June through August.

Figure B.6.1: MOPITT base modeled mean global $\mathrm{OH}$ distribution for each month, May through August 2016. Figures on the left represent the $\mathrm{OH}$ zonal mean molecules $/ \mathrm{cm}^{3}$ across the globe. Figures on the right represent to total column CO concentration in molecules $/ \mathrm{cm}^{2}$. The white contour line represents the MOPITT integrated model global $\mathrm{OH}$ concentration's $80^{\text {th }}$ percentile. 


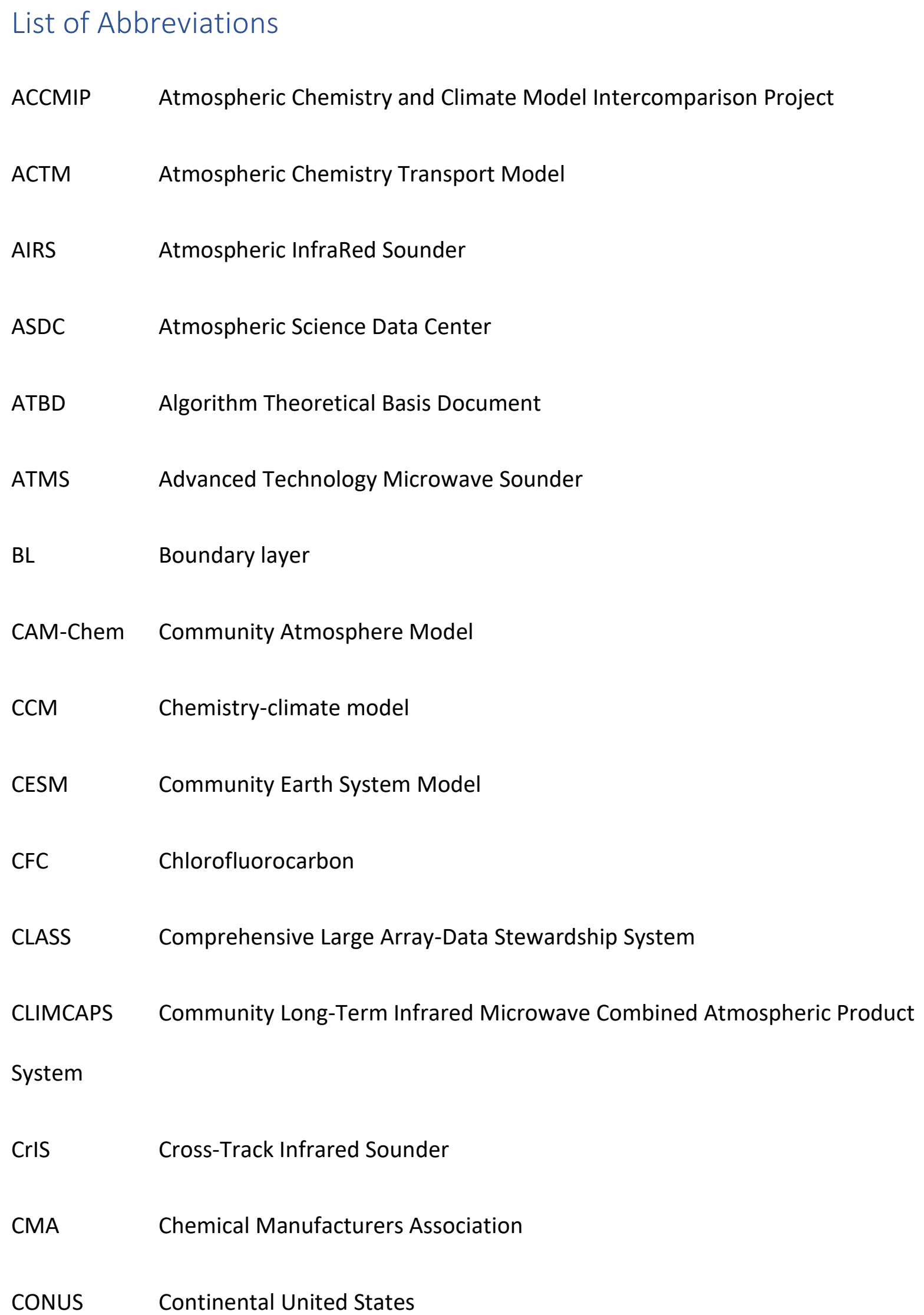




\begin{tabular}{ll} 
CTM & Chemical transport model \\
DAAC & Distributed Active Archive Center \\
DS & Deep Space \\
DOFS & Degrees of freedom for signal (or DFS) \\
ECMWF & European Centre for Medium-Range Weather Forecasts \\
EMAC & ECHAM/MESSy Atmospheric Chemistry model \\
EOSDIS & Earth Observing System Data and Information System \\
FAGE & Fluorescence Array by Gas Expansion \\
FOV & Field of view \\
FSR & Full-spectral-resolution \\
GEOS-FP & GEOS-Forward Processing \\
FTIR & Fourier-Transform Infrared \\
FTS & Fourier Transform Spectrometer \\
GCC & GEOS-Chem Classic \\
& GEOS-Chem High Performance \\
\hline
\end{tabular}




\begin{tabular}{|c|c|}
\hline GES DISC & Goddard Earth Sciences Data and Information Services Center \\
\hline GFDL-CM3 & Geophysical Fluid Dynamics Laboratory CM3 model \\
\hline GMAO & Global Modeling Assimilation Office \\
\hline GOME & Global Ozone Monitoring Experiment \\
\hline IASI & Infrared Atmospheric Sounding Interferometer \\
\hline IAV & Interannual variability \\
\hline ICT & Internal Calibration Target \\
\hline INCA & Interaction with Chemistry and Aerosols model \\
\hline IR & Infrared \\
\hline ITCZ & Intercontinental Convergence Zone \\
\hline JAMSTEC & Japan Agency for Marine-Earth Sciences and Technology \\
\hline JPL & Jet Propulsion Laboratory \\
\hline JPSS & Joint Polar Satellite System \\
\hline LaRC & Langley Research Center \\
\hline LIDAR & Light Detection and Ranging \\
\hline LMC & Length modulating cell \\
\hline LMDZ & Laboratoire de Météorologie Dynamique model \\
\hline
\end{tabular}




\begin{tabular}{|c|c|}
\hline LMR & Length-Modulated Radiometer \\
\hline MCF & Methyl chloroform \\
\hline MERRA & Modern-Era Retrospective Analysis For Research And Applications \\
\hline MIR & Mid-infrared \\
\hline MODIS & Moderate Resolution Imaging Spectroradiometer \\
\hline MOPFAS & MOPITT Operational Fast Forward Model \\
\hline MOPITT & Measurement of Pollution in the Troposphere \\
\hline MSA & Multi-Species Analysis \\
\hline MUSES & Multi-SpEctra, Multi-SpEcies, Multi-Sensors \\
\hline NASA & National Aeronautics and Space Administration \\
\hline NCEP & National Center for Environmental Prediction \\
\hline NDACC & Network for the Detection of Atmospheric Composition Change \\
\hline NDSC & Network for Detection of Stratospheric Change \\
\hline NESDIS & National Environmental Satellite, Data, and Information Service \\
\hline $\mathrm{NH}$ & Northern Hemisphere \\
\hline NIR & Near-infrared \\
\hline NA & National Institute of Water and Atmospheric Research \\
\hline
\end{tabular}




\begin{tabular}{|c|c|}
\hline NOAA & National Oceanic and Atmospheric Administration \\
\hline NPOESS & National Polar-orbiting Operational Environmental Satellite System \\
\hline NPP & NPOESS Preparatory Project \\
\hline NSR & Nominal-spectral-resolution \\
\hline NUCAPS & NOAA Unique Combined Atmospheric Processing System \\
\hline ODS & Ozone-depleting substance \\
\hline$[\mathrm{OH}]_{\mathrm{GM}}$ & Global mean $\mathrm{OH}$ concentration \\
\hline OMI & Ozone Monitoring Instrument \\
\hline OSPO & Office of Satellite Product Operations \\
\hline PAN & Peroxyacyl nitrate \\
\hline PBL & Planetary boundary layer \\
\hline PMC & Pressure modulating cell \\
\hline PMR & Pressure-Modulated Radiometer \\
\hline RADAR & Radio Detection and Ranging \\
\hline RMSE & Root mean square error \\
\hline S5P & Copernicus Sentinel 5 Precursor \\
\hline
\end{tabular}

SCIAMACHY Scanning Imaging Absorption Spectrometer for Atmospheric Cartography 


$\begin{array}{ll}\text { SH } & \text { Southern Hemisphere } \\ \text { SICOR } & \text { Modified SWIR CO retrieval } \\ \text { STAR } & \text { Center for Satellite Application and Research } \\ \text { Suomi-NPP } & \text { Suomi National Polar-orbiting Partnership } \\ \text { SWIR } & \text { Short-wavelength infrared } \\ \text { SZA } & \text { Solar zenith angle } \\ \text { TCCON } & \text { Total Carbon Column Observing Network } \\ \text { TIR } & \text { Thermal infrared } \\ \text { TOA } & \text { Top of atmosphere } \\ \text { TROPOMI } & \text { TROPOspheric Monitoring Instrument } \\ \text { VOC } & \text { Volume mixing ratio } \\ \text { UARS } & \text { Upper Atmosphere Research Satellite } \\ \text { UNFCCC } & \text { United Nations Framework Convention on Climate Change } \\ \text { UV } & \text { Ultraviolet } \\ \text { VMolile organic compound }\end{array}$




\begin{tabular}{|c|c|}
\hline $\mathrm{CH}_{3} \mathrm{CCl}_{3}$ & Methyl chloroform (MCF) \\
\hline $\mathrm{CH}_{4}$ & Methane \\
\hline $\mathrm{CO}$ & Carbon monoxide \\
\hline $\mathrm{H}_{2} \mathrm{O}_{2}$ & Hydrogen peroxide \\
\hline $\mathrm{HCHO}$ & Formaldehyde \\
\hline HCFC & Hydrochlorofluorocarbon \\
\hline $\mathrm{HFC}$ & Hydrofluorocarbon \\
\hline $\mathrm{HNO}_{3}$ & Nitric acid \\
\hline $\mathrm{HO}_{2}$ & Hydroperoxyl radical \\
\hline $\mathrm{HOx}$ & Hydrogen oxides \\
\hline $\mathrm{N}_{2}$ & Nitrogen gas \\
\hline $\mathrm{NO} x$ & Nitrogen oxides \\
\hline $\mathrm{OH}$ & Hydroxyl radical \\
\hline
\end{tabular}




\subsection{Introduction}

With increasing concern on the impacts of greenhouse gases (GHGs), climate change, and regional air quality, scientific studies continue to investigate and understand global atmospheric chemistry. GHGs are naturally removed from the atmosphere through oxidation with the hydroxyl radical $(\mathrm{OH})$, which predominately occurs within the troposphere (Jacob, 2000). However, it is challenging to directly measure $\mathrm{OH}$ concentrations due to the radical's short lifespan and high variability (L. Zhang et al., 2010). Although in-situ measurements of OH are possible, instrument inaccuracy and limitations make directly measuring $\mathrm{OH}$ on a global scale is not currently feasible. Global $\mathrm{OH}$ concentrations are instead inversely calculated through the depletion of trace gases, the most commonly used being methyl chloroform (MCF) (e.g. Bousquet et al., 2005; Brenninkmeijer et al., 1992; Krol \& Lelieveld, 2003; Liang et al., 2017; Montzka et al., 2000, 2011; Patra et al., 2014; Prinn et al., 2001).

In 1987 the Montreal Protocol banned the production and consumption of ozonedepleting substances (ODSs) including chlorofluorocarbons (CFCs) and MCF (Montzka et al., 2011). The now significantly lower global atmospheric MCF hinders its ability to be a reliable trace gas to infer global $\mathrm{OH}$, leading the scientific community to seek alternatives (Wolfe et al., 2019). This study explores carbon monoxide (CO) as an alternative trace gas to inversely calculate $\mathrm{OH}$ through assimilation of observations into the global GEOS-Chem chemical transport model (CTM). There are few existing investigations known to the authors using CO as a sole trace gas to infer OH (Gaubert et al., 2017; Jones, 2019; X. Zhang et al., 2019). 
There are three parts to this study's methods to evaluate $\mathrm{CO}$ as a trace gas to determine $\mathrm{OH}$. In Part 1, global CO total column and profile retrievals from the Measurement of Pollution in the Troposphere (MOPITT), Cross-Track Infrared Sounder (CrIS), and TROPOspheric Monitoring Instrument (TROPOMI) satellite instruments are cross compared against Network for the Detection of Atmospheric Composition Change (NDACC) ground stations and the GEOSChem model. These comparisons are made from May through August with 2016 (MOPITT, NDACC, and GEOS-Chem) and 2019 values (CrIS and TROPOMI) due to data availability limitations. This $\mathrm{CO}$ intercomparison between instruments assesses their precision, accuracy, as well as possible bias and error.

In Part 2, the GEOS-Chem adjoint is used to determine the sensitivities of NDACC locations and a MOPITT $\mathrm{CO}$ assimilation to $\mathrm{CO}$ emissions and the $\left[\mathrm{CH}_{4}+\mathrm{OH}\right]$ chemical reaction rate. This offers insight into the impact these two CO datasets would have on the GEOS-Chem model's output. Finally, in Part 3 the global $\mathrm{OH}$ is determined from the MOPITT CO assimilation and compared against the base GEOS-Chem model global $\mathrm{OH}$. Together, all three parts provide an initial look into the impact of GEOS-Chem $\mathrm{CO}$ assimilation on modeled global $\mathrm{OH}$.

This research revealed that the MOPITT satellite currently provides greatest tropospheric CO total column and profile accuracy when compared against NDACC. Sensitivities from Part 2 demonstrate MOPITT's greater impact on the GEOS-Chem model than the NDACC network. Therefore, MOPITT currently offers the best CO accuracy for assimilation with GEOSChem to infer $\mathrm{OH}$. CrIS provides high resolution $\mathrm{CO}$ profile accuracy only against high elevation NDACC stations, as CrIS's sensitivity is best at elevations above $700 \mathrm{hPa}$ (Smith et al., 2020b). 
TROPOMI has a comparative total column next to MOPITT, however this cannot be confirmed without a proper cross-kernel calculation analysis.

Alone, an assimilation of current CrIS and TROPOMI CO datasets would provide further insight into global $\mathrm{OH}$ restricted to the free troposphere and surface concentrations compared to MOPITT, respectively. However, a future Joint CrIS-TROPOMI product has the potential to offer even greater information into $\mathrm{OH}$ spatial distribution, temporal, and regional trends. The shorter lifetime of CO (months) compared to MCF (years) also has the potential to reveal and better constrain regional $\mathrm{OH}$ distributions and trends, which may improve our understanding of regional air quality. An improved observational bound on $\mathrm{OH}$ abundance would enhance understanding of chemical reaction rates in the atmosphere and improve global atmospheric model calculations for future atmospheric research. 


\subsection{Literature Review}

Over the past half-century, atmospheric scientists have made significant progress in understanding, defining, measuring, and modeling the Earth's atmosphere. Greenhouse gases (GHGs) and other atmospheric constituents impacting Earth's systems and contributing to climate change are in continuous study. Understanding the removal rates of GHGs from the atmosphere is an important factor in predicting the future of Earth's atmospheric state. The main method of removal for certain GHGs, including methane $\left(\mathrm{CH}_{4}\right)$, is through oxidation with the hydroxyl radical $(\mathrm{OH})(\mathrm{Jacob}, 2000)$. This work investigates the potential of using remote sensing observations of carbon monoxide (CO) as a trace gas to constrain global mean hydroxyl radical concentrations $\left([\mathrm{OH}]_{\mathrm{GM}}\right)$. An improved observational bound on $[\mathrm{OH}]_{\mathrm{GM}}$ would enhance understanding of chemical reaction rates in the atmosphere, specifically the lifetime of greenhouse gases like methane, and improve global atmospheric model calculations.

This section contains four subsections containing key concepts and history related to this work, in efforts to provide the reader with the context for understanding the importance and impact of this research. Section 2.1 overviews the Earth's atmospheric structure and the spatial domain of this study. Section 2.2 reviews relevant atmospheric chemistry including the production and depletion of $\mathrm{OH}$ and $\mathrm{CO}$. Section 2.3 reviews the history of determining $\mathrm{OH}$ concentrations, past methods, and historical $\mathrm{OH}$ abundances. Finally, Section 2.4 describes important concepts of remote sensing, radiation, and the atmosphere. 


\subsection{Earth's Atmosphere}

This section contains background knowledge of Earth's atmospheric layers including composition, characteristics, and influences in relation to this study. Components of the atmospheric layers discussed help define the domain of this research, and choice of CO as a potential tracer for $\mathrm{OH}$. Section 2.1.1 reviews basic characteristics of the different atmospheric layers, and Section 2.1.2 discusses atmospheric mixing within and between the layers of interest in this study.

\subsubsection{Atmospheric structure and influences}

The troposphere is the lowermost layer of the atmosphere, spanning to the tropopause 8 to $18 \mathrm{~km}$ above Earth's surface (Jacob, 2000). The air temperature is typically highest at the surface and lowest at the tropopause, with pressures ranging from over $1000 \mathrm{hPa}$ at sea level and decreasing exponentially to about $200 \mathrm{hPa}$ at the tropopause (Jacob, 2000; Liou, 2002). The lowermost few kilometres is referred to as the planetary boundary layer, and has strong interactions with the Earth's surface (Liou, 2002). The top of the boundary layer through to the tropopause is also referred to as the free troposphere (Lelieveld et al., 2016). The troposphere as well as other atmospheric layers are shown in Figure 2.1.1.1 below. Natural and anthropogenic short and long-lived gases and particulates cycle through the troposphere through various sources and sinks. The majority (99.9\%) of the total atmospheric mass resides in the lower layers of the atmosphere, which includes both the troposphere and stratosphere (Jacob, 2000). 
The stratosphere extends from the tropopause to the stratopause at an altitude of about $50 \mathrm{~km}$ and pressure nearing $1 \mathrm{hPa}$ (Jacob, 2000; Liou, 2002). Air temperature increases from the tropopause to the stratopause due to ultraviolet solar radiation absorbed by high concentrations of ozone $\mathrm{O}_{3}$ (the ozone layer) (Jacob, 2000). The next atmospheric layer is the mesosphere, which extends from the stratopause to the mesopause at an altitude of about 80 $\mathrm{km}$. This is followed by the thermosphere then exosphere respectively (Jacob, 2000; Liou, 2002). The layers of the atmosphere can also be addressed as the lower (troposphere), middle (stratosphere and mesosphere), and upper atmosphere (thermosphere and exosphere) (Liou, 2002). This research only discusses the tropospheric and stratospheric domains. The primary focus of this research is within the tropospheric domain as most oxidation by $\mathrm{OH}$ occurs in the troposphere (see Section 2.2).
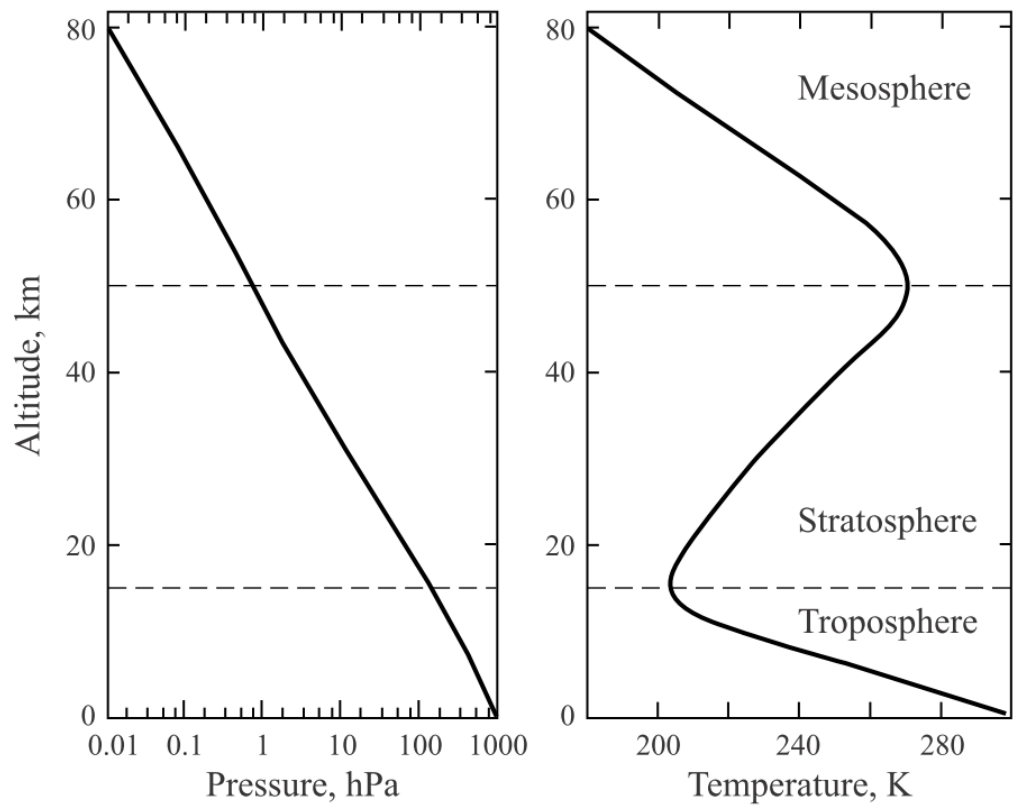

(Jacob, 2000) 
Interactions and exchange between the troposphere and stratosphere change spatially and temporally between seasons, altering the altitude of the tropopause (K.-Y. Wang et al., 2002). The mean tropopause height follows a meridional shape - it is roughly the same across all longitudes. It is highest in the tropics and lowest at the poles, with a sharp gradient at the edge of the extratropics (S. Hu \& Vallis, 2019). The dominant property shaping the high tropical tropopause altitude is the tropics' large optical depth due to abundant water vapour retaining warmer temperatures (decreasing lapse rate) (S. Hu \& Vallis, 2019) and circulation upwelling (Birner, 2010; Haqq-Misra et al., 2011; S. Hu \& Vallis, 2019). Seasonal variations can be attributed to temperature changes affecting optical path, lapse rate, and other parameters such as atmospheric mixing (see Section 2.1.2) (S. Hu \& Vallis, 2019; K.-Y. Wang et al., 2002).

Various $\mathrm{OH}$ studies define the tropospheric domain differently. Lawrence et al. (2001) found that the domains in their surveyed studies are "usually below either the $100 \mathrm{hPa}$ or 200 hPa pressure level, or some sort of latitudinally dependent tropopause." Along with calculative differences, differences in domain can influence the final calculated $\mathrm{OH}$ concentration (Lawrence et al., 2001). This study defines the $\mathrm{OH}$ domain to be between the surface and 100 $\mathrm{hPa}$, as it is a commonly researched domain and would encompass shifts in the tropopause. 


\subsubsection{Atmospheric mixing}

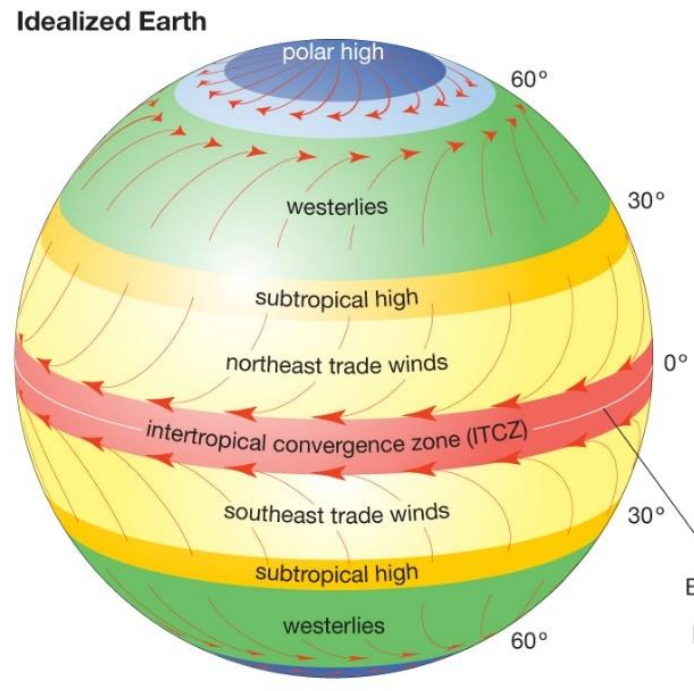

() 2010 Encyclopædia Britannica, Inc.

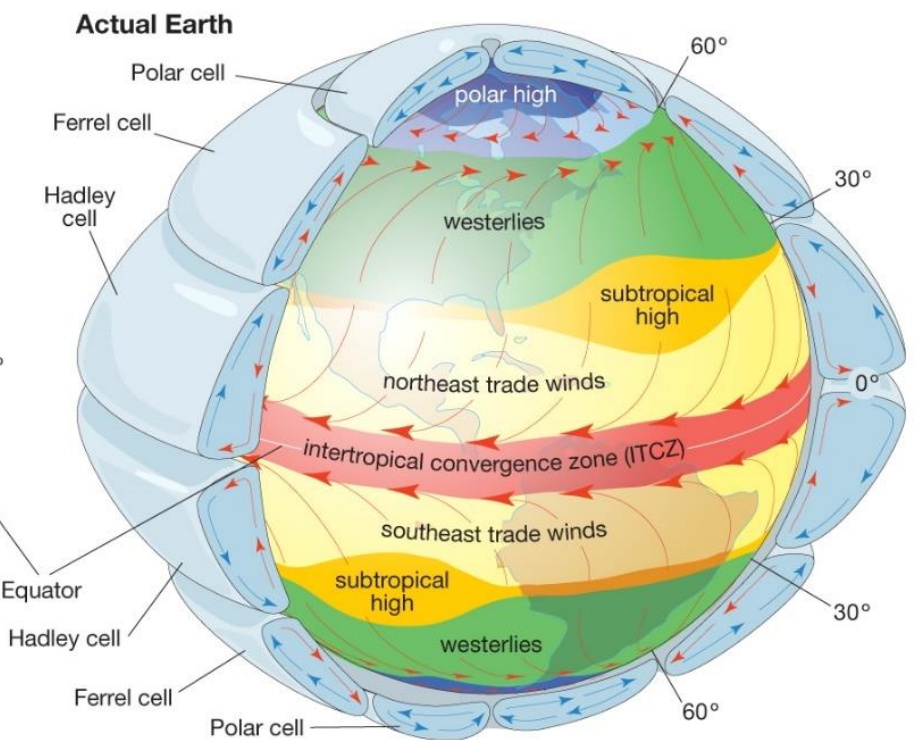

(Encyclopedia Britannica, 2010)

Figure 2.1.2.1: Diagram of atmospheric circulation depicting the three cells of the atmosphere, resulting trade winds, and the intertropical convergence zone (ITCZ). (Encyclopedia Britannica, 2010)

As outlined in the Jacob (2000) atmospheric chemistry textbook, the distribution of trace gases over time in the troposphere and stratosphere depends on lateral and vertical mixing, and their atmospheric lifetime. The textbook explains that lateral mixing depends on winds generated by the pressure-gradient force between high- and low-pressure systems. Upwelling of hot air at the equator, and downwelling of cool air at the poles generate atmospheric circulation (Jacob, 2000). The Coriolis force induced by the Earth's spin divides circulation in the troposphere into three cells per hemisphere as shown in Figure 2.1.2.1: the Hadley cell $\left(0^{\circ}\right.$ to $30^{\circ}$ latitude), a mid-latitude cell $\left(30^{\circ}\right.$ to $60^{\circ}$ latitude) also referred to as the Ferrel cell (Encyclopedia Britannica, 2010), and a polar cell ( $>60^{\circ}$ latitude) (Jacob, 2000). These cells are the primary forces of horizontal and vertical transport (Jacob, 2000). 
Jacob (2000) explains that vertical mixing depends on air turbulence generated from temperature gradients and barometric law. Gravitational separation or molecular diffusion effects of gases are negligible in the troposphere and stratosphere compared to more significant turbulent mixing (Jacob, 2000). As shown in Figure 2.1.2.2, it takes approximately 1 month for gases to mix vertically and zonally within the troposphere. It also shows that within the troposphere, it takes 1 to 2 days for gases to mix well below the planetary boundary layer (PBL) (which ranges 1 to $3 \mathrm{~km}$ above the surface), and 1 week to transport above the PBL.

Mixing across the tropopause occurs at different rates, where tropospheric air may take 5 to 10 years to enter the stratosphere and only 1 to 2 years for stratospheric air to enter the troposphere (Jacob, 2000).

The textbook also introduces the intercontinental convergence zone (ITCZ), a shifting mixing boundary at the equator. Seasonal shifts in January and July are shown in Figure 2.1.2.3. The ITCZ is where the atmosphere of the northern hemisphere $(\mathrm{NH})$ and southern hemisphere (SH) meet, warm, and rise as part of the Hadley cell (Jacob, 2000). Horizontal transport across the ITCZ takes about a year due to lack of thermal forcing (Jacob, 2000).
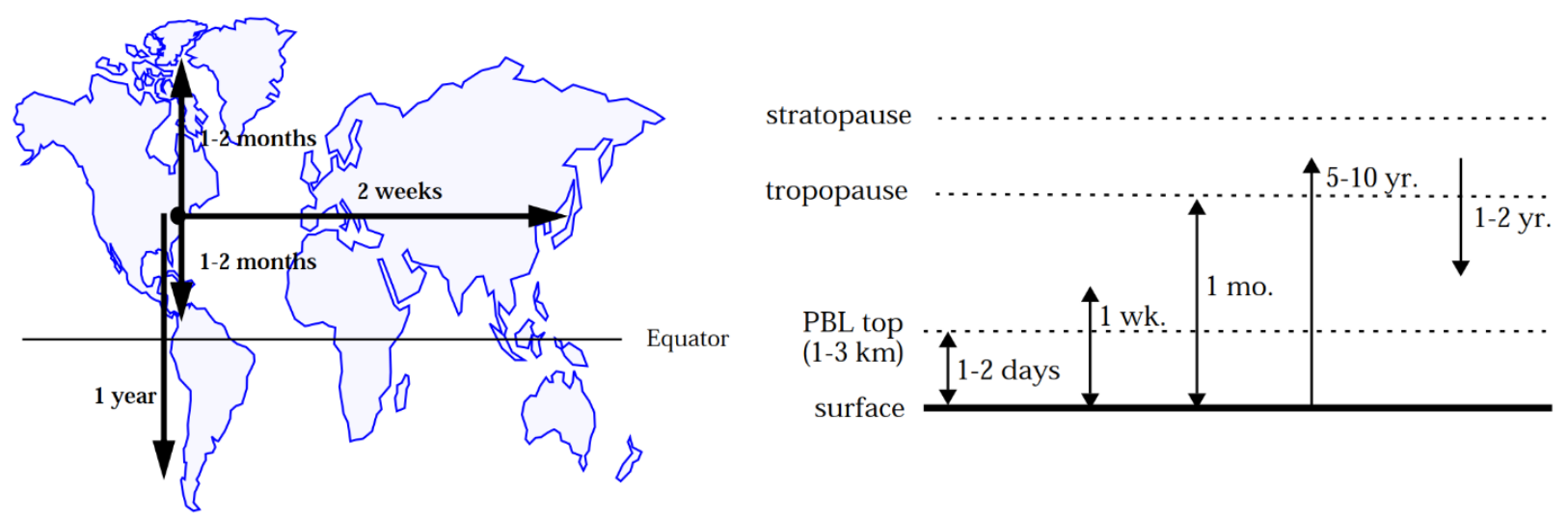

(Jacob, 2000) 
Figure 2.1.2.2: Vertical (left) and horizontal (right) atmospheric mixing times. (Jacob, 2000)
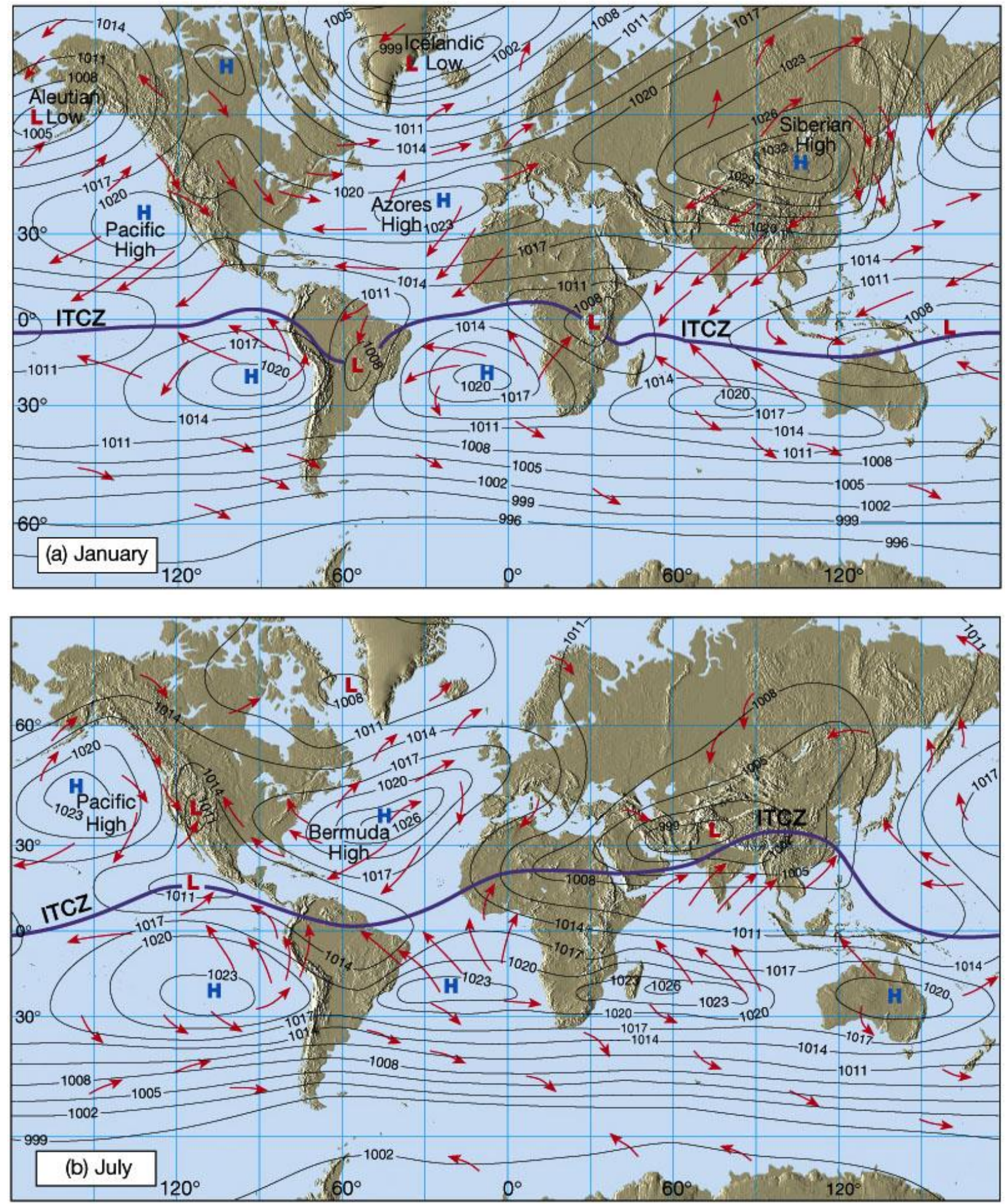

(Lutgens \& Tarbuck, 2001) 


\subsection{Atmospheric Chemistry}

Jacob (2000) defines the atmospheric lifetime (or residence time) of an atmospheric molecule is the average time that it remains in the atmosphere. Particles and gases enter the troposphere through surface emissions, chemical production, and inflow from the stratosphere (Jacob, 2000). The textbook defines the relationship in Equation 1 below, where particles and gases are removed through deposition $(D)$, chemical loss $(L)$, and outflow to the stratosphere $\left(F_{\text {out }}\right)$. Here, the tropospheric lifetime $(\tau)$ is defined as the mass $(m)$ divided by the removal rate $\left(F_{\text {out }}+L+D\right)$. Often $F_{\text {out }}$ is minimal and atmospheric lifetime largely depends on chemical loss and deposition (Jacob, 2000).

$$
\begin{aligned}
& \text { Eqn. (1) } \quad \tau=\frac{m}{F_{\text {out }}+L+D} \\
& \text { where } \quad \tau=\text { lifetime of the tracer } \\
& m=\text { mass of tracer } \\
& F_{\text {out }}=\text { outflow } \\
& L=\text { chemical loss } \\
& D=\text { deposition }
\end{aligned}
$$

Chemical loss of GHGs occurs through oxidation, which can also lead to deposition of certain species. Tropospheric oxidation of $\mathrm{CH}_{4}$ by $\mathrm{OH}$ represents the main $\mathrm{GHG}$ loss pathway 
and it is therefore important to know tropospheric $\mathrm{OH}$ abundance (Lelieveld et al., 2016). $\mathrm{OH}$ abundance is indirectly measured using the inverse relationship between $\mathrm{OH}$ and the reductant species' abundance. The reductant species used to infer $\mathrm{OH}$ is known as the tracer. With $\mathrm{CO}$ being the largest tropospheric $\mathrm{OH}$ sink (Lelieveld et al., 2016), $\mathrm{CO}$ has potential to be used as an OH tracer.

When choosing an $\mathrm{OH}$ tracer, it is important that the species and be well mixed within the atmosphere for inferring global OH (Liang et al., 2017; Wolfe et al., 2019). If the lifetime is too short, then the tracer cannot provide global coverage. If the tracer lifetime is too long, then it may lose sensitivity to variations. For example, the past common $\mathrm{OH}$ tracer methyl chloroform ( $\mathrm{MCF}$ or $\mathrm{CH}_{3} \mathrm{CCl}_{3}$ ) has a long lifetime of approximately 5 years, hindering the ability to observe seasonal variations (Jöckel, 2001). Wolfe et al. (2019) use formaldehyde (HCHO) as an alternative tracer, which has a short lifetime and is not well mixed; however, it is a product of methane which is well mixed. The lifetime of CO ranges from several weeks to several months (Landgraf et al., 2018) with an average lifetime of 2.3 months (Gaubert et al., 2016), making it a potential $\mathrm{OH}$ tracer candidate with sensitivity to seasonal variations.

This section reviews $\mathrm{OH}$ chemical production, loss, and other aspects influencing $\mathrm{OH}$ lifetime and abundance. Section 2.2.1 describes the primary formation of $\mathrm{OH}$ process and associated components. Section 2.2.2 discusses $\mathrm{OH}$ depletion, secondary formation, recycling, and the termination of $\mathrm{OH}$ chain reactions. Section 2.2.3 discusses $\mathrm{OH}$ stability and lifetime within the atmosphere. 


\subsubsection{Primary formation of $\mathrm{OH}$}

The chemical family of hydrogen oxide radicals $\left(\mathrm{HO}_{\mathrm{x}}\right)$ includes $\mathrm{OH}$ and hydroperoxyl radical $\left(\mathrm{HO}_{2}\right)$ (Jacob, 2000). In the troposphere, Jacob explains primary $\mathrm{OH}$ is produced by photolysis through a balance of three reactions below (R1.1 to R1.3). First, solar radiation penetrating through the ozone layer into the troposphere photodissociates ozone into oxygen and electronically excited $O\left({ }^{1} D\right)(R 1.1)$. The radiation flux required to initiate this reaction is in the ultraviolet (UV) with wavelengths between 300 and $320 \mathrm{~nm}$ (Jacob, 2000). Second, a large portion of $\mathrm{O}\left({ }^{1} \mathrm{D}\right)$ interacts with air molecules (designated by $M$ ) to relax to the ground state $\mathrm{O}\left({ }^{3} \mathrm{P}\right)(\mathrm{R} 1.2)$. Third, the remaining portion interacts with water vapour molecules to generate the hydroxyl radical $\mathrm{OH}$ (R1.3).

$$
\begin{gathered}
O_{3}+h v \rightarrow O_{2}+O\left({ }^{1} D\right) \\
O\left({ }^{1} D\right)+M \rightarrow O\left({ }^{3} P\right)+M \\
O\left({ }^{1} D\right)+H_{2} O \rightarrow 2 O H
\end{gathered}
$$

In the Liou (2002) atmospheric radiation textbook, it is further explained that primary $\mathrm{OH}$ production depends on the availability of water vapour, ozone, and radiation. Here, both water vapour and ozone are variable atmospheric gases, unlike the more stable permanent gases such as nitrogen $\left(\mathrm{N}_{2}\right), \mathrm{O}_{2}$, or $\mathrm{CO}_{2}$. Water vapour in the atmosphere varies significantly both spatially and temporally. It depends on local climates, hydrological cycles (evaporation, condensation, precipitation), and atmospheric transport (Liou, 2002). Liou demonstrates that less $\mathrm{OH}$ variability exists in regions with more consistent hydrological cycles and its variability is 
lowest in the equatorial and polar regions. Vertically, water vapour concentrations in the troposphere are significantly higher than in the stratosphere, with about $90 \%$ confined below $500 \mathrm{hPa}$ and highest concentrations within the subtropics below $700 \mathrm{hPa}$ (Liou, 2002).

Most ozone is found in the ozone layer at altitudes around 15 to $30 \mathrm{~km}$, with a maximum concentration around 20 to $25 \mathrm{~km}$ (Liou, 2002). The amount of ozone varies depending on latitude and season, where the amount of available solar radiation influences the production and destruction ozone (Liou, 2002). Additional ozone can also be generated in the troposphere in or near regions of high hydrocarbon and nitrogen oxide ( $\mathrm{NO}_{\mathrm{x}}$ ) pollution due to secondary formation (see Section 2.2.2) (Jacob, 2000). Primary OH production is more abundant in areas of high humidity and only occurs during daylight hours when UV light is available to photodissociate ozone (Jacob, 2000). Therefore, $\mathrm{OH}$ concentrations are highest in the tropics where moisture is high, solar zenith angle is small, and the ozone layer is thin (Jacob, 2000; Lelieveld et al., 2004). Figure 2.2.1.1 below contains plots depicting the higher mean $\mathrm{OH}$ concentrations towards the tropics in May 2016, duplicated by the GEOS-Chem model. This high $\mathrm{OH}$ concentration band shift northwards in the Northern Hemisphere summer. 

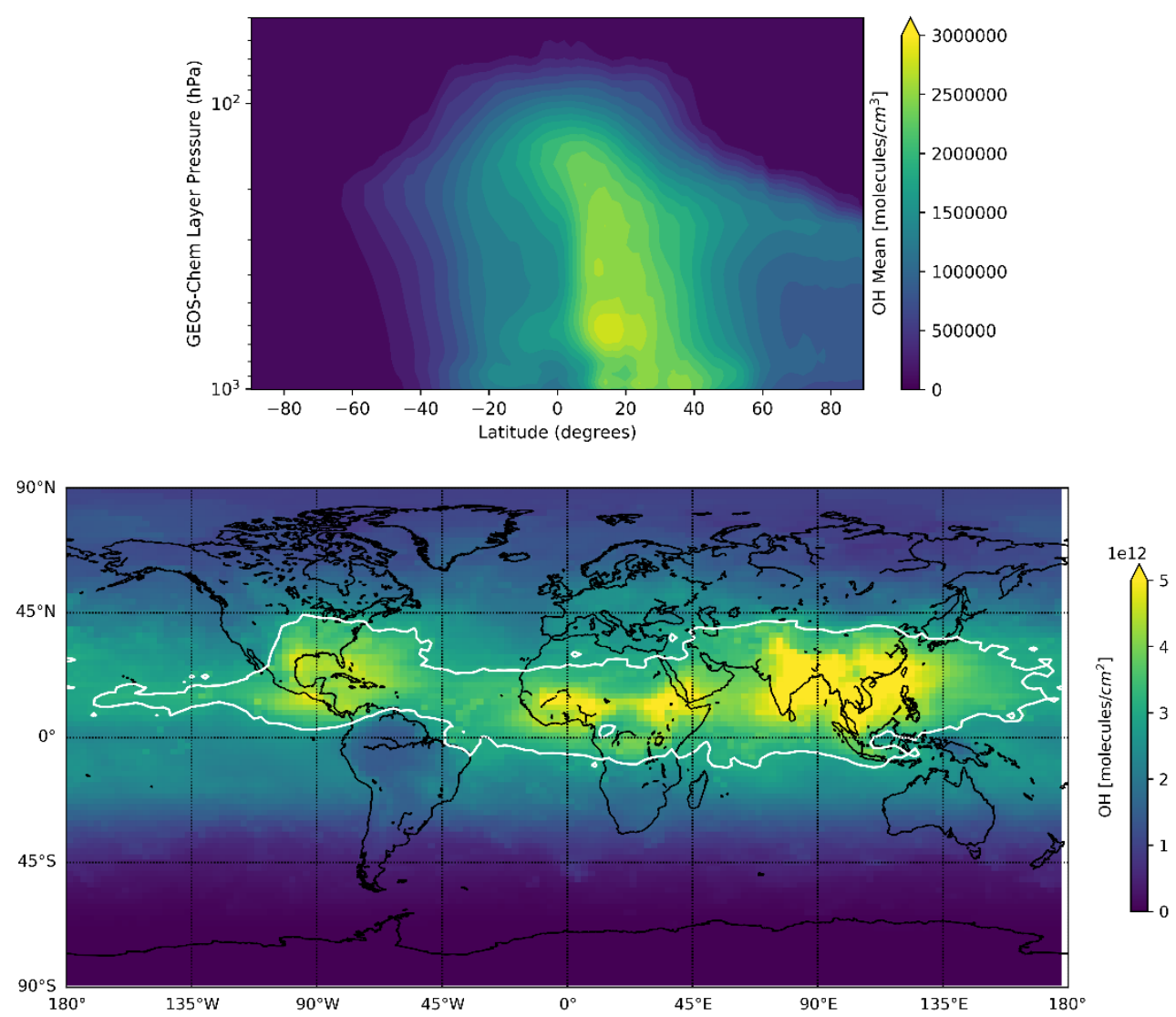

Figure 2.2.1.1: Global spatial distribution of mean $\mathrm{OH}$ concentrations depicted by the GEOS-Chem base model for May 2016. The top plot shows the mean zonal vertical distribution in molecules $/ \mathrm{cm}^{3}$ through the modeled atmosphere. The bottom plot shows horizonal $\mathrm{OH}$ total column distribution in molecules $/ \mathrm{cm}^{2}$, the white contour depicting the approximate $80^{\text {th }}$ percentile.

\subsubsection{OH depletion, secondary formation, recycling, and termination}

Depletion of $\mathrm{OH}$ in the troposphere primarily occurs through oxidation with $\mathrm{CO}$ (Lelieveld et al., 2016) to form carbon dioxide and hydrogen atoms (R2.1) (Jacob, 2000). This reactive hydrogen atom then continues a chain reaction to generate hydroperoxyl radicals by reacting with oxygen and air molecules (R2.2) (Jacob, 2000). Hydroperoxyl radicals can then react with itself to create oxygen and hydrogen peroxide (R2.3) or react with ozone to form oxygen and secondary $\mathrm{OH}$ (R2.4) (Jacob, 2000). Hydrogen peroxide can also generate secondary $\mathrm{OH}$ through photolysis (R2.5) (Jacob, 2000). Alternatively, hydrogen peroxide can react with $\mathrm{OH}$ again (R2.6), continuing the chain reaction (Jacob, 2000). Jacob explains that the chain reaction 
may also be terminated through the deposition of hydrogen peroxide in water droplets due to its high water solubility. It is through this deposition that $\mathrm{HO}$ radicals are removed from the atmosphere (Jacob, 2000; Lelieveld et al., 2004).

$$
\begin{gathered}
\mathrm{CO}+\mathrm{OH} \rightarrow \mathrm{CO}_{2}+\mathrm{H} \\
\mathrm{H}+\mathrm{O}_{2}+\mathrm{M} \rightarrow \mathrm{HO}_{2}+\mathrm{M} \\
\mathrm{HO}_{2}+\mathrm{HO}_{2} \rightarrow \mathrm{H}_{2} \mathrm{O}_{2}+\mathrm{O}_{2} \\
\mathrm{HO}_{2}+\mathrm{O}_{3} \rightarrow \mathrm{OH}+2 \mathrm{O}_{2} \\
\mathrm{H}_{2} \mathrm{O}_{2}+h v \rightarrow 2 \mathrm{OH}^{\mathrm{O}} \rightarrow \mathrm{HO}_{2}+\mathrm{H}_{2} \mathrm{O}
\end{gathered}
$$

The oxidation of hydrocarbons other than $\mathrm{CO}$ follow a similar process but with more steps, where the molecule eventually breaks down to yield CO. Like the carbon monoxide chain reaction, other chain reactions can generate secondary $\mathrm{OH}$ and additional $\mathrm{HO}$ x radicals (Jacob, 2000). The products produced in these chains can take different routes and further decompose either by further oxidation or photolysis (Jacob, 2000). These various reaction paths yield a net consumption of $\mathrm{HO}_{x}$ radicals (Jacob, 2000).

Nitrogen oxides (NOx) are another $\mathrm{OH}$ sink, but they can also induce $\mathrm{OH}$ recycling (Jacob, 2000; Lelieveld et al., 2004). Atmospheric NOx is generated through high temperature oxidation of nitrogen through natural processes such as lightning and microbial processes, and 
anthropogenic combustion - a major source of $\mathrm{NO}_{\mathrm{x}}$ within the troposphere (Jacob, 2000). Jacob outlines their impact through reactions R5.1 to R5.7 explained here. Hydroperoxyl radicals react with nitric oxide to form nitrogen dioxide, another path for secondary $\mathrm{OH}$ formation (R5.1). The nitrogen dioxide can then generate additional ozone (R5.2), allowing for additional primary production (R1.1 to R1.3). $\mathrm{NO}_{x}$ may also react with $\mathrm{OH}$ within the troposphere to produce water soluble nitric acid $\left(\mathrm{HNO}_{3}\right)$, contributing to the removal of $\mathrm{NO}_{x}$ through precipitation (R5.7). However, in the presence of nitrogen oxides an additional three times more $\mathrm{OH}$ can be produced (Jacob, 2000), where NOx is the limiting factor (Lelieveld et al., 2004). Secondary OH formation is also referred to as $\mathrm{OH}$ recycling (Jacob, 2000). A high NOx regime combined with the methane reaction paths above, yield a production of $\mathrm{O}_{3}$ and $\mathrm{HO}$ x radicals (Jacob, 2000). Lelieveld et al. (2004) further explains that in a NOx depleted system, "an increase in reactive carbon enhances $\mathrm{OH}$ loss".

$$
\begin{gathered}
\mathrm{HO}_{2}+\mathrm{NO} \rightarrow \mathrm{OH}+\mathrm{NO}_{2} \\
\mathrm{NO}_{2}+\mathrm{hv} \stackrel{\mathrm{O}_{2}}{\rightarrow} \mathrm{NO}+\mathrm{O}_{3} \\
\mathrm{OH}+\mathrm{O}_{3} \rightarrow \mathrm{HO}_{2}+\mathrm{O}_{2} \\
\mathrm{HO}_{2}+\mathrm{O}_{3} \rightarrow \mathrm{OH}+2 \mathrm{O}_{2} \\
\mathrm{NO}_{+} \mathrm{O}_{3} \rightarrow \mathrm{NO}+\mathrm{O}_{2} \\
\mathrm{NO}_{2}+\mathrm{O} \rightarrow \mathrm{NO}+\mathrm{O}_{2}
\end{gathered}
$$




$$
\mathrm{NO}_{2}+\mathrm{OH}+\mathrm{M} \rightarrow \mathrm{HNO}_{3}+\mathrm{M}
$$

Note that both $\mathrm{HO}_{x}$ and $\mathrm{NO}_{\mathrm{x}}$ can both produce and destroy ozone. In the stratosphere, the balance tips so that ozone is lost through reactions R5.3 to R5.6 due to higher ozone abundance and $\mathrm{O}\left({ }^{3} \mathrm{P}\right)(\mathrm{Jacob}, 2000)$. In the troposphere, reaction R5.3 becomes significantly slower as ozone oxidation competes with carbon monoxide oxidation (R2.1) (Jacob, 2000). Reactions R5.5 and R5.6 become negligible in the troposphere, as reaction R5.1 is significantly faster and there is a limited source of oxygen atoms (Jacob, 2000).

Lelieveld et al. (2016) explains in reactions R6.1 to R6.5 that in the presence of volatile organic compounds (VOCs), $\mathrm{OH}$ is first depleted but then generates secondary $\mathrm{OH}$ through oxidized VOCs (OVOCs). First, a VOC molecule is oxidised by $\mathrm{OH}$ to produce an organic radical and water molecule (R6.1). The radical then progresses to react with oxygen to form organic peroxy radicals (R6.2), which can then progress to react with the hydroperoxyl radical to form one of three sets of products (R6.3 to R6.5). It can generate $\mathrm{OH}$ along with RO and oxygen (R6.4), or $\mathrm{ROOH}$ (R6.3) which can further decompose to produce $\mathrm{OH}$, or generate additional ozone (R6.5) which may produce additional primary OH (R1.1 to R1.3) (Lelieveld et al., 2016). These reactions do not depend on solar radiation and can therefore generate $\mathrm{OH}$ during the night, however modeled nighttime generation was not found to significantly contribute to $\mathrm{OH}$ production on a global scale (Lelieveld et al., 2016).

$$
\begin{gathered}
\mathrm{RH}+\mathrm{OH} \rightarrow \mathrm{R}+\mathrm{H}_{2} \mathrm{O} \\
\mathrm{R}+\mathrm{O}_{2} \rightarrow \mathrm{RO}_{2}
\end{gathered}
$$




$$
\begin{gathered}
\mathrm{RO}_{2}+\mathrm{HO}_{2} \rightarrow \mathrm{ROOH}+\mathrm{O}_{2} \\
\mathrm{RO}_{2}+\mathrm{HO}_{2} \rightarrow \mathrm{RO}+\mathrm{O}_{2}+\mathrm{OH} \\
\mathrm{RO}_{2}+\mathrm{HO}_{2} \rightarrow \mathrm{ROH}+\mathrm{O}_{3}
\end{gathered}
$$

Chemical modeling by Lelieveld et al. (2016) found that the three principal secondary $\mathrm{OH}$ pathways are through reactions with $\mathrm{NO}_{x}$ (R5.1 to R5.6), $\mathrm{O}_{x}(\mathrm{R} 2.4$ and R2.5) and OVOCs (R6.1 to R6.5). In their study, the NOx hydroxyl recycling mechanism was found to dominate in anthropogenically-influenced environments. This agrees with knowledge that the largest atmospheric source of $\mathrm{NO}_{\mathrm{x}}$ in the troposphere is the anthropogenic combustion of fossil fuels (Liou, 2002). However, they also found that $\mathrm{NO} x$ recycling of $\mathrm{OH}$ to be self-limiting within the boundary layer in high pollution areas as $\mathrm{NO}_{2}$ is a large $\mathrm{OH}$ sink, where reaction $\mathrm{R} 5.7$ becomes stronger. More secondary $\mathrm{NO}_{\mathrm{x}}$ formation is found in the free troposphere through the reaction with longer-lived NOx reservoir gases (Lelieveld et al., 2016). Although there are natural sources of $\mathrm{NO}_{x}$ including fires, lightning, soil microbial activity (Murray et al., 2014), the model found OVOC hydroxyl recycling to be dominant over less anthropogenically influenced environments such as forests. Due to the short lifetime of OVOCs, its presence impacts the $\mathrm{OH}$ column rather than surrounding environments (Lelieveld et al., 2016). In marine environments (low NOx and OVOC) their model found the Ox recycling mechanism to be dominant as ozone has a lifetime of several weeks, allowing for long distance transport over oceans.

\subsubsection{OH stability and lifetime}

The global distribution of $\mathrm{OH}$ varies spatially and temporally, influenced by location and seasonal changes. With $\mathrm{OH}$ being highly reactive, it is quickly consumed and recycled within 
seconds making it difficult to measure in space and time (Lelieveld et al., 2016). As discussed in the previous sections, $\mathrm{OH}$ production depends on primary formation and secondary formation. Primary formation is highest in the tropics aligned with higher UV radiation intensity and water vapour availability, and secondary formation is influenced by the presence and balance of NOx, $O_{x}$ and OVOCs (Lelieveld et al., 2004, 2016). The total balance of oxidative power $(G)$ is the sum of primary $(P)$ and secondary $(S) \mathrm{OH}$ formation, or the quotient of $P$ by an $\mathrm{OH}$ efficiency factor ( $r$ ) (Lelieveld et al., 2002). The relationship is represented by the following equation (Lelieveld et al., 2002):

$$
\begin{aligned}
& \text { Eqn. (2) } \quad G=P+S=\frac{P}{1-r} \\
& \text { where } \quad G=\text { oxidizing power } \\
& P=\text { primary } O H \text { formation } \\
& S=\text { secondary } O H \text { formation } \\
& r=O H \text { recycling efficiency }
\end{aligned}
$$

In a chemical model study by Lelieveld et al. (2016), it was found that secondary $\mathrm{OH}$ formation is greater than primary formation as the global mean recycling probability was found to be $67 \%$. They found that out of the three secondary formation categories, the $\mathrm{NO}_{\mathrm{x}}$ recycling mechanism had the greatest impact on global $\mathrm{OH}$ followed by the $\mathrm{O}_{\mathrm{x}}$ and OVOC mechanisms. In marine environments where $\mathrm{NO}_{\mathrm{x}}$ and OVOC concentrations are generally low, they found that $r$ approaches zero (below 40\%) and primary $\mathrm{OH}$ formation becomes more dominant. This would 
imply that $\mathrm{OH}$ concentration would be more sensitive to perturbations in reducing agent concentrations such as $\mathrm{CO}$ (Lelieveld et al., 2016). In regions where $\mathrm{NO}_{\mathrm{x}}$ concentrations are high, they found that $r$ approaches one (well over 50\%) and secondary $\mathrm{OH}$ formation becomes stronger. This would imply that $\mathrm{OH}$ concentration in regions with high fossil fuel use would be less sensitive to perturbations in reducing agent concentrations (Lelieveld et al., 2016). This being said, the boundary layer is more sensitive to local carbon emissions than the free troposphere (Lelieveld et al., 2004, 2016).

Lelieveld et al. (2016) find that the gradient between continental (high $r$ ) and marine (low $r$ ) environments differs between the $\mathrm{NH}$ and $\mathrm{SH}$. In the $\mathrm{NH}$, they determine that continental and marine $\mathrm{OH}$ are similar due to higher ozone and $\mathrm{NO}_{x}$ pollution transportation. In contrast, they determine that the $\mathrm{SH}$ continental $\mathrm{OH}$ is approximately $15 \%$ higher than marine $\mathrm{OH}$ with an overall lower $\mathrm{OH}$ abundance. Zonally, these gradients are larger towards the equator and smaller towards the poles (Lelieveld et al., 2016). Seasonally, the study found higher variations towards the poles in the boundary layer as summer primary production contrasts winter production due to solar radiation differences. During hemispherical winters, they determined that $G$ is buffered by $S$ during when solar radiation (and thus $P$ ) are low. The shifting seasonal position of the ITCZ also affects the NH/SH ratio as it acts as a hemispherical transportation barrier (Lelieveld et al., 2016).

The Lelieveld et al. (2016) study also determined that the free troposphere contains $85 \%$ of tropospheric $\mathrm{OH}$ and has more efficient transport and $\mathrm{OH}$ recycling than the boundary layer. Therefore, global $\mathrm{OH}$ distribution is dominated by the free troposphere (Lelieveld et al., 2016). The $67 \%$ global mean recycling probability, compensating effects of secondary $\mathrm{OH}$ formation, 
and free troposphere abundance together buffer $\mathrm{OH}$ against perturbations from natural and anthropogenic emissions on a global interannual scale (Lelieveld et al., 2016).

Carbon monoxide like carbon dioxide is produced through combustion and other biological and chemical processes. The most significant sources of $\mathrm{CO}$ are fossil fuel combustion, biomass burning, and atmospheric oxidation of methane and other hydrocarbons and non-methane volatile organic compounds (NMVOCs) (Landgraf et al., 2018; Murray et al., 2014). The long lifetime of methane provides a relatively stable background oxidation source of CO (Landgraf et al., 2018), with typical atmospheric CO concentrations at 50 to 200 ppbv (Francis et al., 2017). Oxidation of isoprenes and biomass burning are the main sources of CO in the tropics (Landgraf et al., 2018). Fossil fuel combustion is the main source at mid-latitudes in the Northern Hemisphere (Landgraf et al., 2018). Overall, unpolluted areas typically show smaller CO variance than around polluted areas with greater $\mathrm{CO}$ concentrations (Francis et al., 2017), with the exception of wildfires.

Despite increasing GHGs, recent research suggests that tropospheric interannual variation (IAV) of $[\mathrm{OH}]_{\mathrm{GM}}$ is relatively stable and is well buffered by the various chemical pathways to generate $\mathrm{OH}$ (Lelieveld et al., 2016; Montzka et al., 2011). Due to the production and sink balance, $[\mathrm{OH}]_{\mathrm{GM}}$ has a dependence on the ratio between atmospheric carbon and nitrogen. This relationship is shown in Equation 3 (Murray et al., 2014):

Eqn. (3)

$$
[O H] \propto \frac{J_{O_{3}} q S_{N}}{\left(S_{C}^{3 / 2}\right)}
$$


where

$[\mathrm{OH}]=\mathrm{OH}$ concentration

$J_{O_{3}}=$ mean tropospheric ozone photolysis frequency

$q=$ tropospheric mean specific humidity

$S_{N}=$ tropospheric nitrogen source

$S_{C}=$ tropospheric carbon source

OH concentrations $([\mathrm{OH}])$ are proportional to the tropospheric nitrogen $\left(S_{N}\right)$ and carbon $\left(S_{C}\right)$ ratio, photolysis, and humidity (Murray et al., 2014; Y. Wang \& Jacob, 1998). On a global scale, $\mathrm{OH}$ depleting substances such as $\mathrm{CO}$ and $\mathrm{CH}_{4}$ have increased in parallel with $\mathrm{NO}$ (Lelieveld et al., 2004). The minimal $[\mathrm{OH}]_{G M}$ interannual variation measured in multiple studies (Krol \& Lelieveld, 2003; Montzka et al., 2011; Patra et al., 2014) is likely contributed by tropospheric mixing of these parallel increases (Lelieveld et al., 2004). Simulations by Murray et al. (2014) suggest that mean tropospheric ozone photolysis frequency $\left(U_{O_{3}}\right)$ and tropospheric mean specific humidity $(q)$ variability have a greater impact on $[O H]$ than from emissions on an inter-glacial timescale.

The lifetime of $\mathrm{OH}$ is extremely short relative to other atmospheric constituents, with an average ranging between 1 to 2 seconds (Lelieveld et al., 2016). The lifetime of $\mathrm{OH}$ is calculated by the following equation (Jacob, 2000):

Eqn. (4)

$$
\tau_{O H}=\frac{1}{\sum_{i} k_{i} n_{i}}
$$


where

$$
\begin{aligned}
& \tau_{O H}=\text { lifetime of } O H \\
& k=\text { rate constant } \\
& n=\text { number density } \\
& i=\text { species reacting with } O H
\end{aligned}
$$

Lifetime depends on the number densities $\left(n_{i}\right)$ and rate constants $\left(k_{i}\right)$ of species $i$ reacting with $\mathrm{OH}$ (Jacob, 2000). The spatial distribution of $\tau_{O H}$ can reveal information on balances between $\mathrm{OH}$ and reacting species. The chemical model by Lelieveld et al. (2016) revealed small mean differences between the NH (1.4 s) and SH (1.6 s), and larger differences between mean continental ( $0.3 \mathrm{~s})$ and marine $(0.7 \mathrm{~s})$ boundary layer environments. Their $\mathrm{OH}$ lifetime was found to be shortest over tropical and boreal forests due to biomass burning and abundance of isoprene. Their longest lifetimes were found to be at the tropical tropopause (10 to $20 \mathrm{~s}$ ) due to low temperature (reduced reaction rates) and limited reactants; thus implying that the reactants were already oxidised at lower altitudes, possibly due to higher $\mathrm{OH}$ concentrations and slow rising air parcels along the smaller vertical tropical temperature gradient increasing exposure time. In addition, the study found no significant seasonal cycle.

Due to the short lifetime and variability of $\mathrm{OH}$, it is difficult to directly measure global $\mathrm{OH}$ concentrations (L. Zhang et al., 2010). As a result, global OH concentration and distribution are usually modeled or calculated from an inverse relationship with a reacting species known as a tracer. When selecting a tracer, its primary sink must be oxidation with $\mathrm{OH}$ (Wolfe et al., 2019). MCF is a commonly used tracer to inversely calculate global OH concentrations as approximately $82 \%$ of MCF is oxidised by $\mathrm{OH}$ and its sources are well-known because it is controlled under the Montreal Protocol (Liang et al., 2017). However, due to the decline of MCF 
concentrations, other hydrocarbons are being explored as possible substitutes (see Section 2.3.1). $\mathrm{CO}$ may be a viable alternative tracer as $\mathrm{CO}$ is the largest $\mathrm{OH}$ sink within the troposphere at approximately 39\% (Lelieveld et al., 2016).

\subsection{Historical Context and Importance of $\mathrm{OH}$}

In the 1950s it was discovered that oxidation with $\mathrm{OH}$ occurs in the stratosphere, followed by the discovery in the 1970s of significant $\mathrm{OH}$ oxidation in the troposphere (Jacob, 2000). This section includes the background of $\mathrm{OH}$ detection, past and current research, and historical $\mathrm{OH}$ measurements. Section 2.3.1 summarises the difficulties of measuring $\mathrm{OH}$ concentrations and the need for a new trace gas to indirectly measure $\mathrm{OH}$. Section 2.3.2 summarises research on historical global $\mathrm{OH}$ trends from the last glacial maximum, preindustrial times, and the present.

\subsubsection{History of measuring and calculating $\mathrm{OH}$}

Determining the global spatial and temporal trends of $\mathrm{OH}$ is important as the atmospheric lifetime of GHGs depends on $\mathrm{OH}$ concentrations (Jacob, 2000), and regional trends can help us better understand regional air quality. Global tropospheric $\mathrm{OH}$ is usually compared and expressed as global mean concentration $\left([\mathrm{OH}]_{\mathrm{GM}}\right)$, its interannual variation (IAV), and the interhemispheric gradient or ratio between the northern hemisphere $(\mathrm{NH})$ to southern hemisphere (SH). These values may vary depending on instrument, calculation methodologies, domain, and trace gas used.

It is challenging to directly measure $\mathrm{OH}$ concentrations in the atmosphere due to the radical's short lifespan and high variability (L. Zhang et al., 2010). The most common method for 
measuring in situ $\mathrm{OH}$ is the Fluorescence Array by Gas Expansion (FAGE) technique (Amedro et al., 2012), first developed in the 1980 s by Thomas M. Hard (Hard et al., 1984). FAGE instruments expand ambient air samples in a low pressure cell and measures the $\mathrm{OH}$ concentration from laser induced florescence (Heard \& Pilling, 2003). Accuracy of these instruments is improving, but the disagreement between model concentrations and in situ concentrations can differ up to a factor of 10 (Fittschen et al., 2019). Fittschen et al. (2019) note signal interferences could be attributed to by the presence of other fluorescing species, photolysis of other precursors within the FAGE cell, generation of additional OH during expansion, and knowledge gaps in chemical interactions with biogenic volatile organic compounds (VOCs). Due to the FAGE technique's ongoing development, knowledge gaps, cost, and inability to directly measure on a global scale, global $\mathrm{OH}$ concentrations are determined indirectly (Stone et al., 2012).

The indirect method of quantifying $\mathrm{OH}$ includes the measurement of gases that oxidise with $\mathrm{OH}$. These trace gases must be long lived, their sources well constrained on a global scale, and their primary sink should be their reaction with $\mathrm{OH}$ (Wolfe et al., 2019). The $[\mathrm{OH}]_{\mathrm{GM}}$ is dependant on the oxidation rate of reacting species as shown in Equations 5 and 6 below (Lawrence et al., 2001). The weighting factor is dependant on the reaction rate of $\left(k_{X}\right)$ and mass $\left(M_{X}\right)$ of the reacting gas $X$, where the reaction rate is dependant on temperature $(T)$ and pressure (P) (Lawrence et al., 2001).

Eqn. (5)

$$
[O H]_{G M}=\frac{\sum(W \cdot[O H])}{\sum W}
$$


Eqn. (6)

$$
W=k_{X}(T, P) \cdot M_{X}
$$

where

$$
\begin{aligned}
& {[\mathrm{OH}]_{G M}=\text { global mean } \mathrm{OH} \text { concentration }} \\
& {[\mathrm{OH}]=\text { local } \mathrm{OH} \text { concentration }} \\
& W=\text { weighting factor } \\
& k_{X}=\text { reaction rate with gas } X \\
& T=\text { temperature } \\
& P=\text { pressure } \\
& M_{X}=\text { mass of gas } X
\end{aligned}
$$

Atmospheric MCF was commonly used to determine global mean OH (e.g. Bousquet et al., 2005; Krol \& Lelieveld, 2003; Montzka et al., 2000, 2011; Patra et al., 2014). It has an atmospheric lifetime of 5 years (SPARC, 2013), its sources are exclusively anthropogenic and fairly well-known due to regulations about its production (Jacob, 2000), is primarily removed in the troposphere (SPARC, 2013), and its main atmospheric sink is through $\mathrm{OH}$ oxidation (Jacob, 2000). These characteristics provided fair constraints on $[\mathrm{OH}]_{\mathrm{GM}}, \mathrm{IAV}$ and interhemispheric gradients. However, in 1987 the Montreal Protocol banned the production and consumption of ozone-depleting substances (ODSs) including chlorofluorocarbons (CFCs) and MCF (Montzka et al., 2011). This led to the exponential decay of atmospheric MCF abundance until 1997 (Montzka et al., 2011). By 1998, MCF source emission and interannual change uncertainties were minimised, making MCF a more viable $\mathrm{OH}$ tracer (Montzka et al., 2011). In a study by Montzka et al. (2011), the MCF global burden was estimated using weekly surface MCF 
observations from paired-flask samples collected at nine sites across the globe. From this, the study calculated an estimated IAV in $\mathrm{OH}$ concentrations of $2.3 \pm 1.5 \%$.

As described by Wolfe et al. (2019), the "declining MCF concentrations presage reduced precision for inferred $\mathrm{OH}$ in the coming decade, leading to the community to seek alternatives" (e.g. Krol et al., 2008; Lelieveld et al., 2006; Liang et al., 2017; Y. Zhang et al., 2018). Alternative tracers being studied and investigated to constrain global and local $\mathrm{OH}$ lifetimes and concentrations include methane $\left(\mathrm{CH}_{4}\right)$ (e.g. Dalsøren et al., 2016; Dentener et al., 2003; Holmes et al., 2013; Maasakkers et al., 2019; Montzka et al., 2011; Y. Zhang et al., 2018), formaldehyde (HCHO) (Valin et al., 2016; Wolfe et al., 2019), cosmogenic ${ }^{14} \mathrm{CO}$ (e.g. Brenninkmeijer et al., 1992; Krol et al., 2008), and using multi-species analysis (MSA) combinations (e.g. Jones, 2019; Liang et al., 2017; Spivakovsky et al., 2000; X. Zhang et al., 2019). A summary of some calculated $\mathrm{OH}$ concentrations over the past few decades determined through modeling, MCF measurements, and alternative tracers are summarised in Table 2.3.1.1.

A presented alternative to $M C F$ is the use of $\mathrm{CH}_{4}$ to determine $[\mathrm{OH}]_{G M}$. Atmospheric $\mathrm{CH}_{4}$ meets all three requirements by Wolfe et. al. (2019). It has a lifetime of $9.1 \pm 0.9$ years (Prather et al., 2012) , is well constrained, and its dominant sink is with OH (Holmes et al., 2013; Maasakkers et al., 2019). Montzka et al. (2011) finds that $\mathrm{CH}_{4}$ derived $[\mathrm{OH}]_{\mathrm{GM}} \mathrm{IAV}$ is comparable to that of MCF after 1997. With improving constraints on atmospheric $\mathrm{CH}_{4}$, it is a plausible alternative for future use. Y. Zhang et. al. (2018) proposes that high resolution satellite measured $\mathrm{CH}_{4}$ observations from $\mathrm{CrIS}$ and TROPOMI could provide a $[\mathrm{OH}]_{\mathrm{GM}}$ precision better than $1 \%$, and accuracy of $3 \%$ or $7 \%$ for SWIR and TIR retrievals respectively. However, the long lifetime of $\mathrm{CH}_{4}$ may mask finer $\mathrm{OH}$ distribution characteristics and temporal changes. 
Wolfe et al. (2019) investigates the use of formaldehyde ( $\mathrm{HCHO})$ to improve $\mathrm{OH}$ concentration resolution. $\mathrm{HCHO}$ concentrations are retrievable from earth-observing satellites with daily near-global coverage and is generated in nearly all hydrocarbon lifecycles. Approximately $80 \%$ of $\mathrm{HCHO}$ is sourced from $\mathrm{CH}_{4}$, and it has a lifetime of hours - constraining its atmospheric transport (Wolfe et al., 2019). However, HCHO observations in this study are limited to the remote troposphere, which is $70 \%$ of the total tropospheric mass. Although, their study shoes that $\mathrm{HCHO}^{\prime}$ s shorter lifetime reveals seasonal $\mathrm{NH}$ and $\mathrm{SH}$ differences and regional highs and lows.

Another previously investigated alternative is cosmogenic ${ }^{14} \mathrm{CO}$ (e.g. Brenninkmeijer et al., 1992; Jöckel, 2001; Krol et al., 2008). It is mostly produced in the upper troposphere and stratosphere from cosmic ration from cosmic radiation and the Earth's magnetic field (Krol et al., 2008; MacKay et al., 1963; Pandow et al., 1960). ${ }^{14} \mathrm{CO}$ is also released from nuclear power plants (negligible), and is recycled and re-emitted from biogenic sources (Brenninkmeijer et al., 1992). A regional analysis by $\mathrm{Krol}$ et al. (2008) reveals that ${ }^{14} \mathrm{CO}$ is less sensitive to tropical $\mathrm{OH}$ than MCF. They explain that this is due to ${ }^{14} \mathrm{CO}^{\prime} \mathrm{s}$ shorter lifetime and pressure dependant rate constant, compared to MCF's temperature dependant rate constant. Higher ${ }^{14} \mathrm{CO}$ concentrations in the upper troposphere compared to the surface also contrast MCF and other trace gas sensitivities to $\mathrm{OH}$ (Krol et al., 2008). Using ${ }^{14} \mathrm{CO}$ to determine global $\mathrm{OH}$ is limited by the number of ground station measurements and model transport ability. To the authors' knowledge, there is no satellite ${ }^{14} \mathrm{CO}$ product for global coverage.

Using MSA could reduce $\mathrm{OH}$ abundance uncertainties (Liang et al., 2017). Liang et al. (2017) proposes that a combination of HFC-32, HFC-134a, HFC-152a, and HCFC-22 is 
comparable to MCF and has potential to constrain $\mathrm{OH}$ abundance and its hemispheric gradient. Hydrochlorofluorocarbons (HCFCs) and hydrofluorocarbons (HFCs) are primarily removed from the atmosphere by $\mathrm{OH}$, are long lived gases, have no natural emission sources, and are well constrained through monitoring and reported values from Montreal Protocol parties and the United Nations Framework Convention on Climate Change (UNFCCC) (Liang et al., 2017). X. Zhang et al. (2019) found assimilation of $\mathrm{CO}$, nitrogen oxides ( $\mathrm{NOx}$ ) and ozone $\left(\mathrm{O}_{3}\right)$ satellite observations into the GEOS-Chem model yielded reduced model $[\mathrm{OH}]_{\mathrm{GM}}$. As explained in Section 2.2, these three chemical components play key roles in primary and secondary $\mathrm{OH}$ production and loss. Jones et. al. (2019) expands on this investigation by adding $\mathrm{HNO}_{3}$ and $\mathrm{HCHO}$ to the assimilation, again yielding reduced model $[\mathrm{OH}]_{\mathrm{GM}}$.

$\mathrm{CO}$ is the primary $\mathrm{OH}$ reactant and therefore the largest $\mathrm{OH}$ sink in the troposphere (Jacob, 2000), and has an average lifetime of 2.3 months (Gaubert et al., 2016). With improving constraints on atmospheric $\mathrm{CO}$, it is a plausible alternative to $\mathrm{MCF}$ to determine $[\mathrm{OH}]_{\mathrm{GM}}$. A study by Gaubert et al. (2017) with MOPITT CO concentrations assimilated into a chemistry climate model (CCM) shows higher $\mathrm{CH}_{4}$ oxidation with $\mathrm{OH}$. Over their study period, the MOPITTreanalysis yields lower $[\mathrm{OH}]_{\mathrm{GM}}$ compared to control runs between approximately $6 \times 10^{5}$ and $6.5 \times 10^{5}$ molecules $/ \mathrm{cm}^{3}$ (Gaubert et al., 2017). X. Zhang et. al. (2019) and Jones et al. (2019) assimilations of MOPITT CO concentrations into GEOS-Chem also yield lower $[\mathrm{OH}]_{\mathrm{GM}}$ compared to control runs. X. Zhang et. al. (2019) reports a $0.9 \%$ and $1.8 \%$ lower $[\mathrm{OH}]_{\mathrm{GM}}$ decrease for the November 2009 and July 2010, respectively. Jones et. al. (2019) reports "a modest decrease in OH globally" from January 2016 to February 2017. 
Table 2.3.1.1 summarises select studies investigating $[\mathrm{OH}]_{\mathrm{GM}}$, its IAV, and interhemispheric ratio over different time periods using various models and trace gases. Note that not all studies define $[\mathrm{OH}]_{\mathrm{GM}}$ within the same atmospheric domain. Studies define $[\mathrm{OH}]_{\mathrm{GM}}$ below a specific atmospheric pressure, below a combination of pressures, or below the tropopause. Some do not specifically indicate their domain other than just the tropopause, which may have varying spatial domains depending on time of year. The $[\mathrm{OH}]_{G M}$ of each study are comparable between models and tracers used, demonstrating a solid general understanding of $\mathrm{OH}$ and global atmospheric chemistry.

Most studies also state if their $[\mathrm{OH}]_{\mathrm{GM}}$ is an airmass, mass, or volume weighted mean. An airmass weighted mean more heavily weights $\mathrm{OH}$ concentrations towards the surface, increasing importance within the lower troposphere and normalising the vertical distribution. A mass weighted mean more heavily weights towards the tracer gas mass distribution. This normalises the $[\mathrm{OH}]_{G M}$ between the different tracer gases for comparison, but skews $\mathrm{OH}$ distribution between tracers depending on each tracer's spatial distribution (differences in lifetime, and vertical and horizontal transport) (Wolfe et al., 2019). A volume weighted mean more heavily weights towards the upper troposphere, a representative global mean distribution if the vertical and horizontal grids do not contain the same volume.

IAV between studies vary over different time scales but are within the same magnitude. This speaks for $\mathrm{OH}$ being well buffered against atmospheric changes as mentioned in Lelieveld et al. (2016) and Montzka et al. (2011). The $\mathrm{OH}$ interhemispheric ratio varies between studies, showing a disagreement over the years whether more $\mathrm{OH}$ exists within the Northern or 
Southern Hemisphere. This may also depend on the defined domain of the $\mathrm{NH}$ and $\mathrm{SH}$, whether it is defined by the equator or the ITCZ.

This study is an initial look into the potential of using new CO satellite retrievals to determine $\mathrm{OH}$. To our knowledge, Gaubert et al. (2017), X. Zhang et. al. (2019) and Jones et. al. (2019) are the only assimilation studies where CO is used as a sole tracer gas. These previous studies used satellite products with limited ability to distinguish $\mathrm{CO}$ concentrations in the vertical dimension, which can contain important information separating the CO sources from its sinks. Using $\mathrm{CO}$ to determine $[\mathrm{OH}]_{\mathrm{GM}}$ may provide greater spatial and temporal $\mathrm{OH}$ characteristics due to its significantly shorter lifetime than $\mathrm{CH}_{4}$, but longer lifetime than $\mathrm{HCHO}$. The ability to retrieve global CO measurements from satellites also offers a greater potential to constrain $\mathrm{OH}$ distribution than ${ }^{14} \mathrm{CO}$. Using MSA to determine global $\mathrm{OH}$ is a promising venture and can incorporate superior chemical relationships when assimilated into atmospheric models. However, MSA can be computationally expensive for investigating $\mathrm{OH}$ over larger timescales. Assimilating a higher resolution CrIS or TROPOMI CO data product with daily global coverage into an atmospheric model may provide greater insight to vertical and horizontal global $\mathrm{OH}$ distribution, while also providing more information about sources and sinks. 
Table 2.3.1.1: Summary of select global $\mathrm{OH}$ abundance, interannual variations and interhemispheric values determined in previous journal articles and reports

\begin{tabular}{|c|c|c|c|c|c|c|c|}
\hline Study & Model Used & $\begin{array}{c}\text { Species } \\
\text { Used/Compared }\end{array}$ & Study Period & Domain & $\begin{array}{c}\text { Mean Global OH } \\
\text { Molecules } / \mathrm{cm}^{3}\end{array}$ & OH IAV & $\begin{array}{c}\text { Interhemispheric } \\
\text { OH Ratio } \\
\text { (NH/SH ratio) }\end{array}$ \\
\hline $\begin{array}{c}\text { (Brenninkmeijer } \\
\text { et al., 1992) }\end{array}$ & Box & $\mathrm{MCF},{ }^{14} \mathrm{CO}^{\mathrm{a}}$ & $\begin{array}{c}\text { Jun. } 1989- \\
\text { Feb. } 1991 \\
\end{array}$ & troposphere & $7.7 \times 10^{5}$ & - & 1.03 \\
\hline $\begin{array}{c}\text { (Spivakovsky et } \\
\text { al., 2000) }\end{array}$ & CTM & $\begin{array}{c}\mathrm{O}_{3}, \mathrm{H}_{2} \mathrm{O}, \mathrm{NO}_{\mathrm{t}}, \mathrm{CO}, \\
{ }^{14} \mathrm{CO}^{\mathrm{a}} \text { and } \\
\text { hydrocarbons }\end{array}$ & 1978-1996 & $\begin{array}{l}\text { Below } 100 \mathrm{hPa} \\
\text { (within } \pm 32^{\circ} \\
\text { latitude) } \\
\text { Below } 200 \mathrm{hPa} \\
\text { (elsewhere) }\end{array}$ & b $11.6 \times 10^{5}$ & - & 1.00 \\
\hline $\begin{array}{c}\text { (Montzka et al., } \\
\text { 2000) }\end{array}$ & Box & $\mathrm{MCF},{ }^{14} \mathrm{CO}^{\mathrm{a}}$ & 1998-1999 & troposphere & {$[11 \pm 2] \times 10^{5}$} & - & \\
\hline $\begin{array}{c}\text { (Prinn et al., } \\
\text { 2001) }\end{array}$ & Box & MCF & $1978-2000$ & troposphere & $\mathrm{b}[9.4 \pm 1.3] \times 10^{5}$ & - & 0.88 \\
\hline $\begin{array}{c}\text { (Krol \& } \\
\text { Lelieveld, 2003) }\end{array}$ & $\begin{array}{c}\text { TM3 + DISORT } \\
\text { (CTM) }\end{array}$ & MCF & $1978-2000$ & troposphere & $\begin{array}{c}\text { e } 9.6 \times 10^{5}(\sim 1980) \\
\text { e } 10.5 \times 10^{5}(\sim 1990) \\
\text { e } 9.2 \times 10^{5}(\sim 2000)\end{array}$ & - & 0.98 \\
\hline $\begin{array}{c}\text { (Bousquet et al., } \\
\text { 2005) }\end{array}$ & $\begin{array}{c}\text { LMDZ-INCA } \\
\text { (GCM) }\end{array}$ & MCF & $1980-2000$ & troposphere & ${ }^{c}[9.8 \pm 0.9] \times 10^{5}$ & $8.5 \pm 1.0 \%$ & 0.85 \\
\hline $\begin{array}{c}\text { (Krol et al., } \\
2008)\end{array}$ & $\begin{array}{l}\text { TM5 } \\
\text { (CTM) }\end{array}$ & ${ }^{14} \mathrm{CO}$ & $\begin{array}{l}\text { Jan. } 2005- \\
\text { Jul. } 2005\end{array}$ & $\begin{array}{c}\text { Surface } \\
\text { to } 500 \mathrm{hPa}\end{array}$ & $\begin{array}{c}{ }^{\mathrm{c}} 2 \times 10^{5} \text { to } \\
{ }^{\mathrm{c}} 3 \times 10^{5} \\
\text { (regional mean } \mathrm{OH} \text { ) }\end{array}$ & - & - \\
\hline \multirow{3}{*}{$\begin{array}{c}\text { (Montzka et al., } \\
\text { 2011) }\end{array}$} & Box & MCF & $2000-2005$ & \multirow{3}{*}{ troposphere } & \multirow{3}{*}{-} & $2.3 \pm 1.2 \%$ & \multirow{3}{*}{-} \\
\hline & \multirow[b]{2}{*}{ Box } & $\begin{array}{c}\mathrm{CH}_{4} \\
\text { (constant emissions) }\end{array}$ & $1985-2008$ & & & $2.1 \pm 1.8 \%$ & \\
\hline & & $\begin{array}{c}\mathrm{CH}_{4} \\
\text { (variable emissions from } \\
\text { biomass burning and } \\
\text { wetlands included) }\end{array}$ & $1998-2007$ & & & $1.8 \pm 1.2 \%$ & \\
\hline $\begin{array}{c}\text { (Voulgarakis et } \\
\text { al., 2013) }\end{array}$ & $\begin{array}{c}\text { ACCMIP } \\
\text { Multi-Model }\end{array}$ & 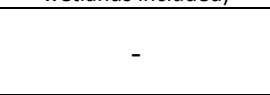 & 2000 & Below $200 \mathrm{hPa}$ & ${ }^{b}[11.1 \pm 1.8] \times 10^{5}$ & - & - \\
\hline \multirow{3}{*}{$\begin{array}{l}\text { (Naik et al., } \\
\text { 2013) }\end{array}$} & \multirow{3}{*}{$\begin{array}{c}\text { ACCMIP } \\
\text { Multi-Model }\end{array}$} & \multirow{3}{*}{-} & 1850 & Below $200 \mathrm{hPa}$ & $\mathrm{b}^{\mathrm{b}}[11.3 \pm 1.7] \times 10^{5}$ & \multirow{3}{*}{-} & $1.13 \pm 0.09$ \\
\hline & & & 1980 & Below $200 \mathrm{hPa}$ & ${ }^{\mathrm{b}}[10.8 \pm 1.6] \times 10^{5}$ & & $1.25 \pm 0.10$ \\
\hline & & & 2000 & Below $200 \mathrm{hPa}$ & ${ }^{\mathrm{b}}[11.1 \pm 1.6] \times 10^{5}$ & & $1.28 \pm 0.10$ \\
\hline $\begin{array}{c}\text { (Patra et al., } \\
\text { 2014) }\end{array}$ & $\begin{array}{c}\text { JAMESTC } \\
\text { ACTM } \\
\end{array}$ & MCF & 2004-2011 & unspecified & - & - & $0.97 \pm 0.12$ \\
\hline
\end{tabular}




\begin{tabular}{|c|c|c|c|c|c|c|c|}
\hline $\begin{array}{c}\text { (Lelieveld et al., } \\
\text { 2016) }\end{array}$ & $\begin{array}{l}\text { EMAC } \\
\text { (GCM) }\end{array}$ & - & 2013 & $\begin{array}{l}\text { Surface to } \\
\text { tropopause }\end{array}$ & $\begin{array}{l}\text { d } 11.3 \times 10^{5} \\
\text { b } 11.1 \times 10^{5}\end{array}$ & - & $\begin{array}{c}\neq 1.20 \text { (equator) } \\
* 1.25 \text { (equator) } \\
\ddagger 1.13 \text { (ITCZ) }\end{array}$ \\
\hline $\begin{array}{c}\text { (Dals } \emptyset \text { ren et al., } \\
\text { 2016) }\end{array}$ & Oslo CTM3 & $\mathrm{CH}_{4}$ & $1970-2012$ & troposphere & $\begin{array}{c}8.7 \times 10^{5} \text { to } \\
13.4 \times 10^{5}\end{array}$ & - & 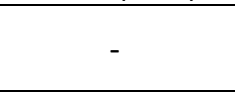 \\
\hline \multirow{2}{*}{$\begin{array}{c}\text { (Liang et al., } \\
\text { 2017) }\end{array}$} & Box & MCF & - & troposphere & ${ }^{\mathrm{c}} 11.2 \times 10^{5}$ & \multirow[b]{2}{*}{-} & 0.9 to 1.1 \\
\hline & Multiple CCMs & $\begin{array}{c}\text { MCF, } \text { HFCs }^{a} \text { and } \\
\text { HCFCs }^{a}\end{array}$ & 2000 & Below $200 \mathrm{hPa}$ & $\begin{array}{l}{ }^{c} 10.1 \times 10^{5} \text { to } \\
{ }^{c} 13.0 \times 10^{5}\end{array}$ & & - \\
\hline $\begin{array}{c}\text { (Gaubert et al., } \\
\text { 2017) }\end{array}$ & $\begin{array}{l}\text { CESM/CAM- } \\
\text { Chem } \\
(\mathrm{CCM})\end{array}$ & $\mathrm{CO}$ & 2002-2013 & unspecified & $\begin{array}{l}{ }^{\mathrm{b}} 6 \times 10^{5} \text { to } \\
{ }^{\mathrm{b}} 6.5 \times 10^{5}\end{array}$ & - & - \\
\hline \multirow[b]{2}{*}{$\begin{array}{c}\text { (Turner et al., } \\
\text { 2018) }\end{array}$} & \multirow[b]{2}{*}{$\begin{array}{l}\text { GDFL-CM3 } \\
\text { (CCM) }\end{array}$} & \multirow[b]{2}{*}{-} & \multirow{2}{*}{$\begin{array}{l}\text { Preindustrial } \\
\text { control years } \\
3000-6000\end{array}$} & \multirow[b]{2}{*}{$300-800 \mathrm{hPa}$} & \multirow[b]{2}{*}{-} & $\begin{array}{c}1.0 \% \text { (over } \\
3000 \text { yrs) }\end{array}$ & \multirow[b]{2}{*}{-} \\
\hline & & & & & & $\begin{array}{c}3.8 \pm 0.8 \% \\
\text { (decadal } \\
\text { average) }\end{array}$ & \\
\hline $\begin{array}{c}\text { (Maasakkers et } \\
\text { al., 2019) }\end{array}$ & $\begin{array}{l}\text { GEOS-Chem } \\
\text { (CTM) }\end{array}$ & $\mathrm{CH}_{4}$ & 2010-2015 & troposphere & $\tau_{C H_{4}}^{O H}=10.8 \pm 0.4$ years & $\begin{array}{c}-0.2 \% \pm \\
0.8 \% \\
\end{array}$ & - \\
\hline $\begin{array}{c}\text { (Wolfe et al., } \\
\text { 2019) }\end{array}$ & 0-D Box & $\mathrm{HCHO}$ & 2016-2017 & $\begin{array}{c}70 \% \text { of } \\
\text { tropospheric } \\
\text { mass } \\
\end{array}$ & ${ }^{f}[10.3 \pm 2.5] \times 10^{5}$ & - & $0.89 \pm 0.06$ \\
\hline \multirow{4}{*}{$\begin{array}{c}\text { (X. Zhang et al., } \\
\text { 2019) }\end{array}$} & \multirow{4}{*}{$\begin{array}{l}\text { GEOS-Chem } \\
\text { (CTM) }\end{array}$} & \multirow{2}{*}{$\mathrm{O}_{3}, \mathrm{CO}$, and $\mathrm{NO}$} & Nov 2009 & \multirow{4}{*}{ troposphere } & ${ }^{\mathrm{c}} 10.5 \times 10^{5}$ & \multirow{4}{*}{-} & \multirow{4}{*}{-} \\
\hline & & & July 2010 & & c $12.8 \times 10^{5}$ & & \\
\hline & & \multirow{2}{*}{$\mathrm{CO}$} & Nov 2009 & & ${ }^{c} 11.8 \times 10^{5}$ & & \\
\hline & & & July 2010 & & ${ }^{c} 13.6 \times 10^{5}$ & & \\
\hline (Jones, 2019) & $\begin{array}{l}\text { GEOS-Chem } \\
\text { (CTM) }\end{array}$ & $\begin{array}{c}\mathrm{CO}, \mathrm{O}_{3}, \mathrm{NO}_{2} \\
\mathrm{HNO}_{3} \text {, and } \mathrm{HCHO}\end{array}$ & $\begin{array}{l}\text { Jan. } 2016- \\
\text { Feb. } 2017\end{array}$ & troposphere & c $12 \times 10^{5}$ & - & - \\
\hline
\end{tabular}

a Species is not used to calculate $\mathrm{OH}$, but rather is compared to other species.

${ }^{\mathrm{b}}$ Air mass weighted

${ }^{\mathrm{c}}$ Mass weighted

dVolume weighted

e Approximate 3-year average

${ }^{\mathrm{f}}$ Tropospheric column mean $\mathrm{OH}\left(\mathrm{X}[\mathrm{OH}], \mathrm{cm}^{-3}\right)$ 


\subsubsection{Historical Tropospheric Oxidative Capacity}

Research comparing present atmospheric oxidation capacity to preindustrial times and the last glacial maximum investigate possible historical trends. Murray et al. (2014) infers that multi-millennial global environmental change affects tropospheric oxidative capacity due to its dependences on meteorological, emissions, and stratospheric conditions. These historical changes can help scientists understand chemical, climatic, and ecological consequences (Murray et al., 2014). However, past long-term knowledge is limited and is determined through modeling (Murray et al., 2014).

Murray et al. (2014) further explains that tropospheric oxidation capacity from $\mathrm{OH}$, ozone, and $\mathrm{H}_{2} \mathrm{O}_{2}$ have been modeled back to preindustrial times and the last glacial maximum in various studies. Over modeled glacial-interglacial periods, tropospheric oxidative capacity is sensitive to tropospheric water vapour, lightning NOx emissions, and stratospheric ozone (Murray et al., 2014). They explain that these studies vary by method in using different 1-D to 3D modeling, meteorology, chemistry, and assimilation sources to generate their model outputs. Murray et al. summarises that studies from 1989 to 2013 estimated OH concentrations at the last glacial maximum to be $25 \%(+10 \%$ to $+56 \%$ range) higher than preindustrial times. The higher oxidation capacity further into the past appears logical due to lower hydrocarbon and CO concentrations (Murray et al., 2014).

When comparing preindustrial to modern times, there is a large uncertainty in $\mathrm{OH}$ oxidation capacity ranging from $-31 \%$ to $+60 \%$ (Murray et al., 2014). Naik et. al. (2013) found there is little change in the $[\mathrm{OH}]_{\mathrm{GM}}$ over the past 150 years despite large regional changes due to buffering factors enhancing $\mathrm{OH}$ despite rising pollution. Unlike earlier studies, Murray et al. 
(2014) generated a 3-D tropospheric OH oxidation capacity model output including the influence of stratospheric ozone. Their updated modeled tropospheric mean $\mathrm{OH}$ was found to be similar between the last glacial maximum and preindustrial times at $+0.5 \pm 12 \%$. In addition, their modeled present values show $+7.0 \pm 4.3 \%$ compared to preindustrial times. This relatively small change in $\mathrm{OH}$ from preindustrial to present day can be attributed to the linear relationship between $\mathrm{OH}$ and emissions discussed in Section 2.2.3. Since $\mathrm{OH}$ concentration depends on the $S_{N} /\left(S_{C}^{3 / 2}\right)$ ratio, and industrialisation has increased both $S_{N}$ and $S_{C}$, it is logical that the change in $[\mathrm{OH}]_{\mathrm{GM}}$ is relatively small (Murray et al., 2014; Naik et al., 2013; Thompson et al., 1993).

In recent decades Dalsøren et al. (2016) calculates a growth in $[\mathrm{OH}]_{\mathrm{GM}}$, from an observed $8 \%$ decrease in $\mathrm{CH}_{4}$ lifetime from 1970 to 2012 . Their study agrees that the $S_{N} /\left(S_{C}^{3 / 2}\right)$ emission ratio, specific humidity, and total ozone column play key factors in atmospheric oxidation capacity. These changes are found to agree with most bottom-up model studies, but not with top-down MCF or ${ }^{14}$ CO studies (Dalsøren et al., 2016). Naik et. al. (2013) also observes a $3.5 \pm 2.2 \%$ increase in mean $\mathrm{OH}$ from 1980 to 2000 , leading to a decrease in $\mathrm{CH}_{4}$ lifetime. These recent studies suggest $[\mathrm{OH}]_{\mathrm{GM}}$ has been growing since preindustrial times, although there is no consensus on the amount of growth. Through three different CTM models and observed MCF inferred OH IAV, Holmes et. al. (2013) projects $[\mathrm{OH}]_{\mathrm{GM}}$ to continue to grow into the year 2100 with a calculated $2.2 \pm 1.8 \%$ (10\% uncertainty) decrease in $\mathrm{CH}_{4}$ lifetime. Determining these large-scale trends is important in making inferences for forecasting possible future global $\mathrm{OH}$ trends and impacts. 


\subsection{Remote sensing, radiation, and the atmosphere}

This section overviews how interactions between radiation and the atmosphere are used for the remote sensing of $\mathrm{CO}$ and other atmospheric characteristics. Atmospheric composition can be observed by remote sensing through the use of satellite or ground-based instruments. Remote sensing can be active like the use of lasers in Light Detection and Ranging (LIDAR) and microwaves in Radio Detection and Ranging (RADAR); or it can be passive by measuring direct or reflected solar radiation or thermal emissions. The instruments in this study use passive remote sensing techniques, namely spectroscopy and radiometry. Satellite instruments use detectors to record upwelling radiation from Earth and reflected solar radiation that has passed through the atmosphere. Ground-based instruments record solar radiation that has passed through the atmosphere from space or downwelling thermal emissions from the atmosphere. These instruments generally report concentrations in total column and profile values. A total column is a scalar value of the total concentration from the surface to the top of the atmosphere in molecules per unit area. A profile is a vector representing concentrations between defined vertical levels from the surface to the top of the atmosphere. In Section 2.4.1 the basic remote sensing principles are explained. Section 2.4.2 further elucidates the remote sensing of $\mathrm{CO}$ and discusses different types of interferences.

\subsubsection{Remote sensing principles}

Within the atmosphere particles and gases absorb, scatter, and emit varying amounts of different wavelengths at varying efficiencies (Liou, 2002). By knowing the portion of radiation absorbed, scattered, and emitted by the atmosphere, the abundance of specific gases can ultimately be determined from the observing instrument signal (Liou, 2002). Equation 7 
(Rodgers, 2000) illustrates this relationship, where the signal $\left(\boldsymbol{y}, \widehat{\boldsymbol{b}}, \boldsymbol{x}_{a}, \boldsymbol{c}\right)$ can be transformed by an inverse function $(\boldsymbol{R})$ to determine the specified target profile $(\widehat{\boldsymbol{x}})$. To obtain global CO total column and profile concentrations $(\widehat{x})$, instruments infer CO concentrations from measured infrared (IR) radiation (Liou, 2002). However, the inverse function is complex and non-linear and requires the use of radiative transfer theories and light scattering principles (Jacob, 2000; Liou, 2002; Rodgers, 2000).

Eqn. (7)

$$
\widehat{x}=\boldsymbol{R}\left(\boldsymbol{y}, \widehat{b}, x_{a}, c\right)
$$

where

$$
\begin{aligned}
& \boldsymbol{y}=\boldsymbol{f}(\boldsymbol{x}, \boldsymbol{b})+\boldsymbol{\epsilon} \\
& \widehat{\boldsymbol{x}}=\text { target profile (vector) } \\
& \boldsymbol{R}=\text { inverse or retrieval method } \\
& \boldsymbol{y}=\text { vector valued forward function of } \boldsymbol{x} \text { and } \boldsymbol{b} \\
& \boldsymbol{x}=\text { unknown state vector, species of interest } \\
& \boldsymbol{b}=\text { parameters not included in } \boldsymbol{x} \\
& \boldsymbol{\epsilon}=\text { experimental error term } \\
& \widehat{\boldsymbol{b}}=\text { best estimate of forward function parameters } \\
& \boldsymbol{x}_{a}=\text { a priori estimate of } \boldsymbol{x} \\
& \boldsymbol{c}=\text { remaining parameters affecting the retrieval } \\
& \text { but not the forward function }
\end{aligned}
$$


As the Sun's and Earth's radiation passes through the atmosphere, it interacts with gas molecules and other particles (Jacob, 2000). Specific wavelengths can be absorbed by a gas molecule if it can increase its internal energy level (electronic state) without disassociation (photoionization) or decomposition (Jacob, 2000; Liou, 2002). The spectra allowing these electronic and vibrational transitions are largely in the ultraviolet (UV), visible, and IR wavelengths (Liou, 2002). The textbook by Jacob (2000) explains that greenhouse gases absorb in the infrared part of the spectrum, at wavelengths between $5-50 \mu \mathrm{m}$, generating vibrational and vibrational-rotational transitions. This energy is then re-emitted at a fraction, which can be absorbed again by other molecules (Jacob, 2000). The textbook notes that the absorption efficiency changes with temperature and pressure. The opacity of the atmosphere to a specific wavelength corresponds to the concentration of the absorbing molecules (see Figures 2.4.1.1 and 2.4.1.2) (Jacob, 2000).

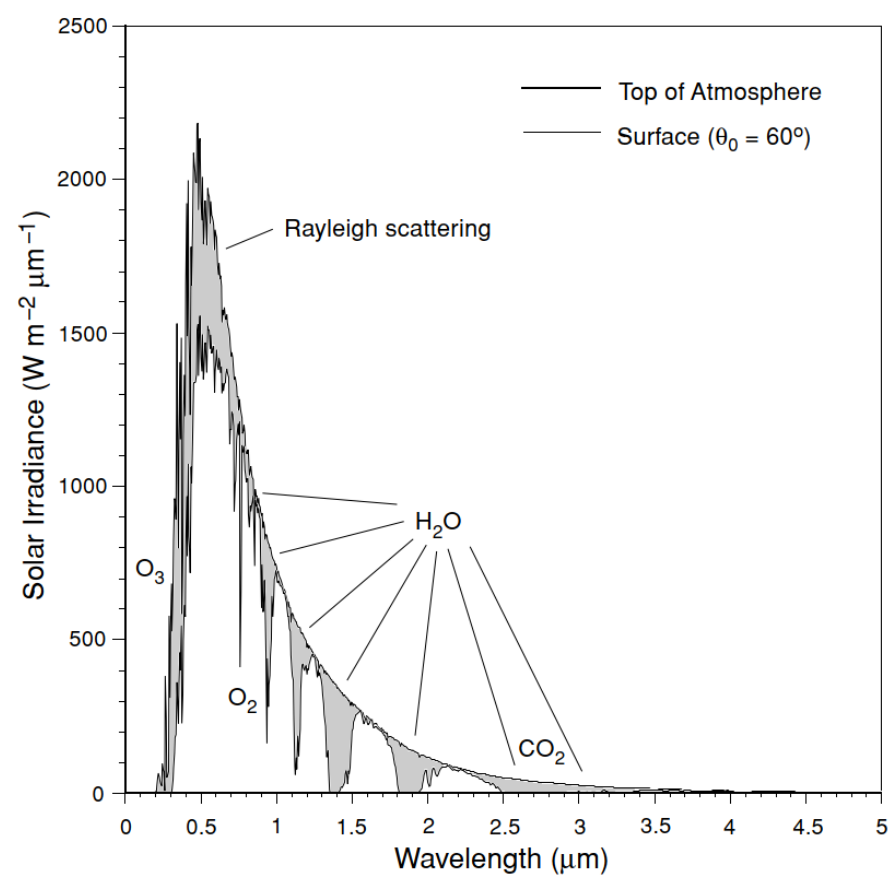

(Liou, 2002) 
Figure 2.4.1.1: Liou (2002) solar irradiance curve depicting absorption and scattering by difference atmospheric species.

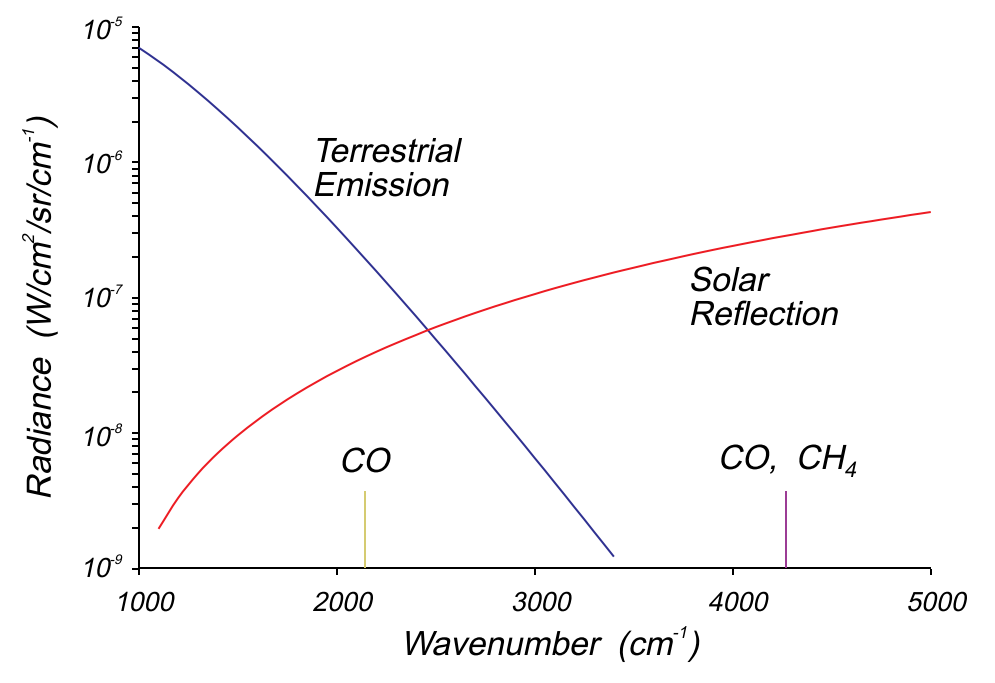

(Gille \& NCAR MOPITT Team, 1996)

Figure 2.4.1.2: Solar reflection and terrestrial emission in comparison to the observed MOPITT instrument CO absorption spectra. Figure retrieved from the MOPITT Algorithm Theoretical Basis Document (ATBD), August 1996.

Spectrometer satellite instruments measure the radiation flux emitted by a unit surface area of the Earth from the top of the atmosphere (Liou, 2002). Each type of gas within the atmosphere absorbs specific wavelengths, creating absorption lines across the radiation spectrum like a fingerprint (Liou, 2002). The width of these absorption lines increases linearly with air density, and consequently provides information on the vertical profile distribution of the corresponding gas (Liou, 2002). The higher the wavelength resolution of the measuring instrument, the greater potential for a higher resolution profile (Jacob, 2000). However, the absorption lines of the various atmospheric gases and particles overlap (Jacob, 2000). To differentiate them from each other, algorithms are required to obtain estimates of concentration (the algorithm is referred to as the "retrieval algorithm") (Jacob, 2000). To 
further complicate the retrieval, IR radiation measurements are also impacted by scattering effects (Jacob, 2000).

Due to the complexity of $\boldsymbol{R}$, passive observation retrievals of atmospheric components generally employ the use of radiative transfer models. These models iteratively calculate radiances to match the observed instrument radiances to infer the target gas, by varying atmospheric characteristics (Rodgers, 2000). Replacing the forward function with a linearized forward model results in Equation 8 (Rodgers, 2000). The first term of the equation $\left(\boldsymbol{R}\left[\boldsymbol{F}\left(\boldsymbol{x}_{a}, \widehat{\boldsymbol{b}}\right), \widehat{\boldsymbol{b}}, \boldsymbol{x}_{a}, \boldsymbol{c}\right]-\boldsymbol{x}_{a}\right)$ is the bias, described as "the error that would result from a simulated retrieval using a simulated error-free measurement of the a priori state computed with the forward model" (Rodgers, 2000). The second term $\left(\boldsymbol{A}\left(\boldsymbol{x}-\boldsymbol{x}_{a}\right)\right)$ represents the smoothing term, where the profile is weighted by the sensitivity of the retrieval to the true state. The third term $\left(\boldsymbol{G}_{y} \boldsymbol{\epsilon}_{y}\right)$ is the retrieval error, due to the total measurement error (Rodgers, 2000).

Eqn. (8) $\widehat{x}-\boldsymbol{x}_{a}=\boldsymbol{R}\left[\boldsymbol{F}\left(\boldsymbol{x}_{a}, \widehat{\boldsymbol{b}}\right), \widehat{\boldsymbol{b}}, \boldsymbol{x}_{a}, \boldsymbol{c}\right]-\boldsymbol{x}_{a}+\boldsymbol{A}\left(\boldsymbol{x}-\boldsymbol{x}_{a}\right)+\boldsymbol{G}_{y} \boldsymbol{\epsilon}_{y}$

where $\quad \boldsymbol{F}=$ forward model

$$
\begin{aligned}
& \boldsymbol{A}=\text { averaging kernel matrix (sensitivity of the retrieval to true state) } \\
& \boldsymbol{G}_{y}=\text { sensitivity of retrieval to the measurement } \\
& \boldsymbol{\epsilon}_{y}=\text { measurement error }
\end{aligned}
$$


Modelling this process on a global scale can be computationally expensive and time consuming. Omission or approximation of less significant parameters, or complete removal complicating parameters can speed up the process at the expense of data quality (Landgraf et al., 2018). For example, some atmospheric instruments such as CrIS and TROPOMI (see Sections 3.1.2 and 3.1.3) include simplified algorithms within their models to compensate for cloud effects (Landgraf et al., 2018; Smith \& Barnet, 2019). The MOPITT instrument (see Section 3.1.1) instead filters out all observations containing clouds, removing these data points completely (Deeter, MOPITT Algorithm Development Team, et al., 2017). Using only clear sky radiances simplifies calculations as most radiation emitted is from the Earth's surface rather than from scattering effects (Landgraf et al., 2018). Some important impactful atmospheric parameters include the effects of scattering and absorption of radiation by other atmospheric particles than the target.

\subsubsection{Remote sensing of CO and interferences}

Remote sensing of CO, like other gases, has a unique spectral signature. Liou (2002) explains that CO has a symmetric vibrational mode and linear diatomic rotational freedom. When CO transitions from vibrational ground state $(v=0)$ to electronic state $(v=1)$, it has a fundamental vibrational band at $2.34 \mu \mathrm{m}$ (wavenumber 4150 to $4350 \mathrm{~cm}^{-1}$ ) (Liou, 2002). When CO transitions from vibrational $v=0$ to $v=2$, it has a first-overtone band at $4.67 \mu \mathrm{m}$ (wavenumber 2000 to $2300 \mathrm{~cm}^{-1}$ ) (Liou, 2002). These create CO's unique spectral signature. Figure 2.4.2.1 shows the first CO overtone band at $4.67 \mu \mathrm{m}$, where the two separate peaks are a product of its two degrees of rotational freedom. 
The $2.34 \mu \mathrm{m}$ spectral band is within the short-wave IR (SWIR) spectral range, which is part of the solar spectrum (Landgraf et al., 2018). It also falls under the near IR (NIR) spectral range (Deeter et al., 2013). However, CO is a weak absorber compared to other absorbers within the SWIR spectrum such as $\mathrm{H}_{2} \mathrm{O}$ and $\mathrm{CH}_{4}$ (Landgraf et al., 2018). It primarily provides information about the total column (Deeter et al., 2013), but its uniform sensitivity is impeded due to lengthened light paths impacting optical depth (Deeter et al., 2013; Landgraf et al., 2018). This makes CO detection more difficult. SWIR measurements are sensitive to total column of $\mathrm{CO}$, and does not provide information on vertical profile distribution (Landgraf et al., 2018).

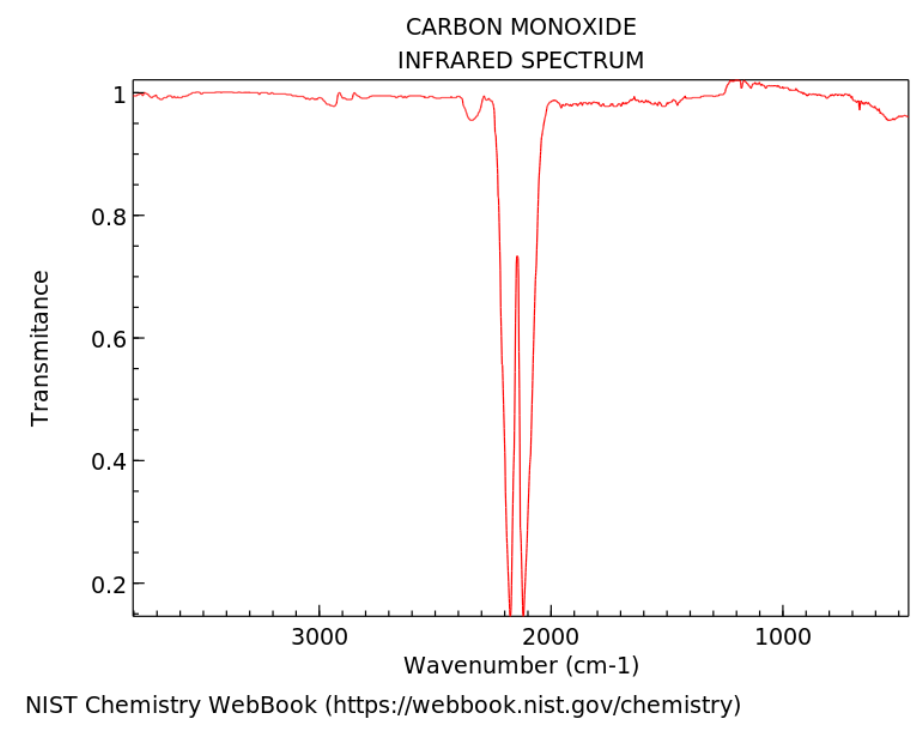

(Linstrom, 1997)

Figure 2.4.2.1: NIST Chemistry WebBook carbon monoxide first-overtone band.

To acquire a vertical profile, more vertical information is required within the spectral band. Carbon monoxide's $4.67 \mu \mathrm{m}$ spectral feature is within the thermal IR (TIR) spectrum (Deeter et al., 2013; Francis et al., 2017). Upwelling thermal radiation originates from the Earth 
and different levels in the atmosphere, where varying concentrations of $\mathrm{CO}$ are present with significant enough opacity (Francis et al., 2017). Therefore, TIR measurements can provide information on $\mathrm{CO}$ profiles using this thermal profile and $\mathrm{CO}$ distribution, along with water vapour profiles, surface temperature and surface emissivity (Francis et al., 2017). For TIR measurements, peak sensitivity is within the middle troposphere, and variable sensitivity within the lower troposphere depending on thermal contrast and resolution (Landgraf et al., 2018). NIR (SWIR) measurements can also be combined with TIR measurements to provide a "multispectral" CO retrieval, enhancing lower troposphere profile retrievals (eg. Deeter et al., 2013; D. Fu et al., 2016).

Radiation scattering and absorbance by clouds and aerosols interfere with remote sensing sensitivity (Liou, 2002). Liou (2002) explains the amount of radiation scattered or absorbed by the atmosphere is described as the optical depth. When radiation reaches a particle, scattering of radiation can occur through reflection, refraction, or diffraction (Liou, 2002). The larger the particle the higher the scattering efficiency (Liou, 2002). Hence, aerosols and cloud particles have high scattering efficiencies due to their large size compared to IR wavelengths (Liou, 2002). Scattering by gas molecules (Rayleigh scattering) in IR is not as efficient as they are significantly smaller than IR wavelengths (Liou, 2002).

Clouds have scattering effects, are efficient IR radiation absorbers (Jacob, 2000), and regularly cover more than $50 \%$ of the Earth's surface (Liou, 2002). As a result, they decrease the measurement sensitivity of CO below the cloud (Landgraf et al., 2018) and are "the largest source of scene-dependant uncertainty in satellite sounding observations" (Smith \& Barnet, 2019). The Liou (2002) textbook explains that cloud composition is important to determine 
their scattering and absorption properties. The text illustrates that their interactions with IR radiation depend on particle concentration, particle size distribution, particle shape, cloud thickness and cloud geometry. Cloud particles can be liquid water droplets (average $8 \mu \mathrm{m}$ radius) or ice crystals (average $42 \mu \mathrm{m}$ radius) (Liou, 2002). Cirrus clouds are composed of ice crystals, are present in the upper troposphere and lower stratosphere, and are normally transparent in the thermal IR spectrum (Liou, 2002).

Aerosols have scattering effects and can also absorb radiation in the visible and near-IR (Liou, 2002). Aerosol concentrations decrease with height due to their mass, and smaller particles remain suspended for longer periods of time (Jacob, 2000). Their effective absorption depends on composition, size and vertical profile (Liou, 2002). The Liou (2002) textbook states that natural sources of aerosols include volcanic dust, forest fire smoke, sea spray, windblown dust (for example, desert sands), and small chemically produced particles. Anthropogenic sources of aerosols include particles emitted through combustion (Liou, 2002). Largest aerosol concentrations are found by urban and desert areas (Liou, 2002). The text also notes that aerosols can also act as a condensing surface and increase cloud formation and affect cloud particle size, also known as the indirect aerosol effect.

Another source of interference is from various other gas species with absorption lines overlapping CO. Comparatively, CO is a minor absorber of near IR radiation (Liou, 2002). Water vapour is the prime absorber of near-IR radiation, and has absorption lines over the entire IR spectrum (Liou, 2002). Other overlapping species include $\mathrm{CO}_{2}\left(2000-2400 \mathrm{~cm}^{-1}\right), \mathrm{O}_{3}(2000-$ $\left.2300 \mathrm{~cm}^{-1}\right), \mathrm{N}_{2} \mathrm{O}\left(2100-2300 \mathrm{~cm}^{-1}\right)$, and $\mathrm{CH}_{4}\left(4000-4600 \mathrm{~cm}^{-1}\right)$ (Liou, 2002). To single out CO, MOPITT enhances its instrument sensitivity to $\mathrm{CO}$ specifically using gas correlation radiometry 
(see Section 3.1.1). Other satellite instruments such as the CrIS retrieve interfering species simultaneously, which is then incorporated into their retrieval algorithm to determine CO (see Section 3.1.2) (Smith \& Barnet, 2019).

$\mathrm{CO}$ and other species retrievals are also subject to multiple types of error including smoothing error, model parameter error, forward model error, and retrieval noise (Rodgers, 2000). Rearrangement of Equation 8 into error terms is shown in Equation 9 (Rodgers, 2000). Rodgers (2000) clarifies that observing instruments are unable to retrieve spatial fine structure and thus require smoothing. The error resulting from statistical smoothing is known as the smoothing error, shown as the first term in Equation $9\left(\left(\boldsymbol{A}-\boldsymbol{I}_{n}\right)\left(\boldsymbol{x}-\boldsymbol{x}_{a}\right)\right)$. The second term $\left(\boldsymbol{G}_{y} \boldsymbol{K}_{b}(\boldsymbol{b}-\widehat{\boldsymbol{b}})\right)$ is the forward model parameter error. The third term $\left(\boldsymbol{G}_{y} \Delta \boldsymbol{f}\left(\boldsymbol{x}, \boldsymbol{b}, \boldsymbol{b}^{\prime}\right)\right)$ is the forward model error, which is determined from the true state and is dependant on the accuracy of the modeled physics (Rodgers, 2000). The final term $\left(\boldsymbol{G}_{y} \boldsymbol{\epsilon}\right)$ is the retrieval noise, where the measurement noise is generally random and unbiased.

$$
\begin{array}{ll}
\text { Eqn. (9) } & \widehat{\boldsymbol{x}}-\boldsymbol{x}=\left(\boldsymbol{A}-\boldsymbol{I}_{n}\right)\left(\boldsymbol{x}-\boldsymbol{x}_{a}\right) \\
& +\boldsymbol{G}_{y} \boldsymbol{K}_{b}(\boldsymbol{b}-\widehat{\boldsymbol{b}}) \\
& +\boldsymbol{G}_{y} \Delta \boldsymbol{f}\left(\boldsymbol{x}, \boldsymbol{b}, \boldsymbol{b}^{\prime}\right) \\
& +\boldsymbol{G}_{\boldsymbol{y}} \boldsymbol{\epsilon}
\end{array}
$$

where $\quad \boldsymbol{I}_{n}=$ identity matrix of rank $n$ (from the profile retrieval)

$\boldsymbol{K}_{b}=$ sensitivity of the forward model to the forward parameters

$\boldsymbol{b}^{\prime}=$ forward function parameters ignored in forward model 


$$
\boldsymbol{\epsilon}=\text { measurement noise }
$$

These errors are categorised as random or systematic errors, depending on how they impact precision and accuracy (Rodgers, 2000). Smoothing error and model parameter errors are considered to be systematic errors as they vary with state (Rodgers, 2000). Retrieval noise is a random error as it is not correlated to states or time (Rodgers, 2000). The overall errors of different observing instrument retrievals depend on their individual instrument uncertainty, a priori assumptions, and model calculation employed. For example, the MOPITT instrument error is most subject to "variability of instrument noise, geophysical noise, thermal contrast, and CO loading" (Deeter et al., 2015). 


\subsection{Methodology}

This methodology section has been divided into two major components: an overview of the datasets and simulation tools used for this study (Section 3.1), and a three part methodology (Sections 3.2 to 3.4). Section 3.1 describes the functionality and retrieval/calculation methods of each instrument. Sections 3.2 to 3.4 outline the methodology of the three parts of this study. Together, these three parts are employed to determine whether the various $\mathrm{CO}$ remote sensing observations could be used to improve global and regional estimates of $\mathrm{OH}$ abundance in the troposphere.

In Part 1, CO satellite observations from MOPITT, CrIS, and TROPOMI are cross evaluated against ground-based NDACC observations and the global GEOS-Chem modeled atmospheric state. This is to assess the differences in precision, accuracy, and vertical sensitivity of the CO total column and profiles. In Part 2, the GEOS-Chem adjoint model is used to determine the sensitivities of the NDACC network and MOPITT CO assimilation to CO emissions and the $\left[\mathrm{CH}_{4}+\mathrm{OH}\right]$ reaction rate. These sensitivities provide insight to the initial model response, how assimilated $\mathrm{CO}$ observations impact the modeled $\mathrm{CO}$ emissions and $\mathrm{OH}$ variability. In Part 3, satellite CO observations are assimilated into the GEOS-Chem Adjoint to generate modeled $\mathrm{OH}$ concentration outputs. These $\mathrm{OH}$ outputs are then compared against the GEOS-Chem base $\mathrm{OH}$ concentration to assess the initial impact of MOPITT CO assimilation. 

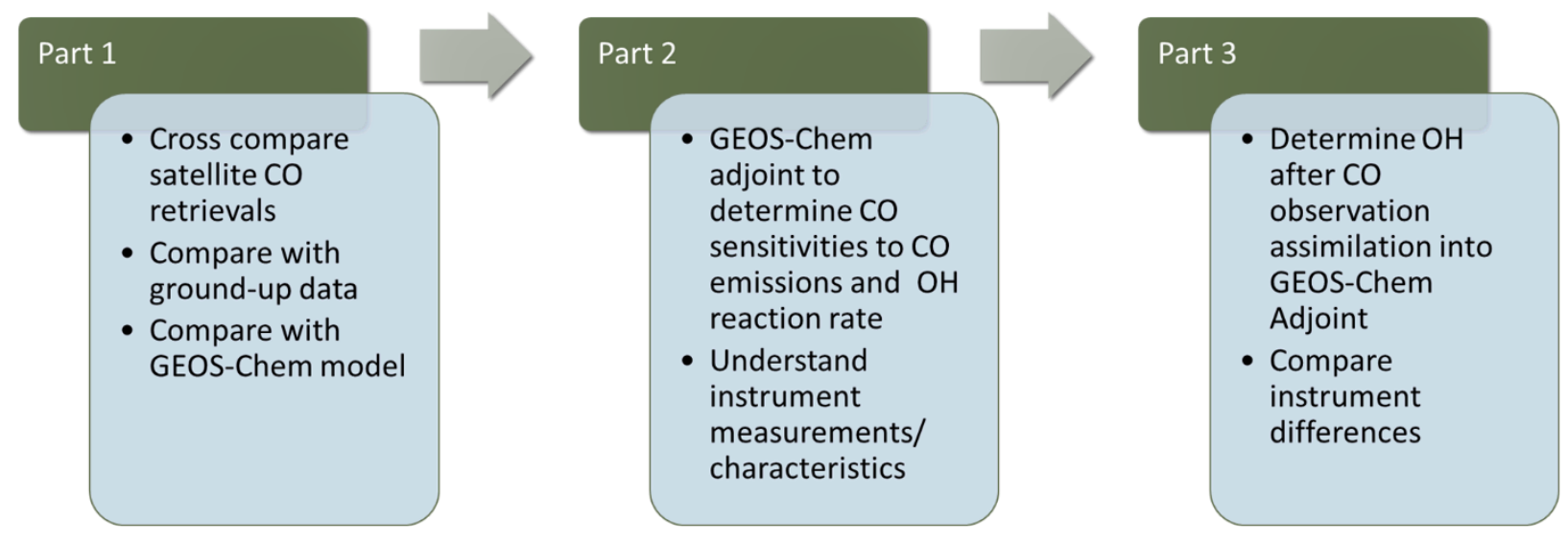

\subsection{Datasets and Simulation Tools}

In this study, CO total columns and profile data is collected from satellite instruments, ground stations, and a global chemical transport model is used to simulate CO concentrations. Data from satellite instruments including the Measurement of Pollution in the Troposphere (MOPITT), Cross-Track Infrared Sounder (CrIS), and TROPOspheric Monitoring Instrument (TROPOMI) are used. Ground station data is retrieved from Fourier-Transform Infrared (FTIR) spectrometers at Network for the Detection of Atmospheric Composition Change (NDACC) stations. The GEOS-chem model is used to simulate global $\mathrm{CO}$ and $\mathrm{OH}$ concentrations. The GEOS-Chem Adjoint is used to determine each observing platforms' sensitivities to the $\mathrm{OH}$ reaction rate and emissions, and also as a platform to prototype data assimilation of the above satellite products.

Data products from satellite instruments are commonly categorized into data product levels. Level 0 data often refers to calibration values and raw radiance data measured by the instrument. Processing of Level 0 data produces Level 1 data products, which are the calibrated 
radiances measured by the instrument. Level 1 data are then processed into Level 2 data, which generally contains the targeted information along the geospatial position of the satellite field of view. In the cases studied here, Level 2 data includes CO total column or profile concentrations. Level 3 data products collect and constitute Level 2 data into a global gridded map. All satellite data used for analysis in this study are Level 2 data products. Satellite and surface instruments measuring atmospheric characteristics such as CO concentration also typically include additional application information for analysis and filtering, such as data quality indicators, error data, a priori profile data, averaging kernel matrices, and degrees of freedom of signal (DOFS or DFS) for each retrieval (Barnet, 2019; Copernicus Sentinel-5P (processed by ESA), 2018; Francis et al., 2017; Landgraf et al., 2018; NDACC, 2021; Smith \& Barnet, 2019; Ziskin, 2019). Each of these parameters are further explained in Section 3.2.

The following subsections describe the different instruments used for data collection. Sections 3.1.1, 3.1.2, and 3.1.3 contain descriptions of the MOPITT, CrIS, and TROPOMI satellite instruments, respectively. These subsections include details of instrument operation and of the retrieval algorithms for CO. Section 3.1.4 overviews the Joint CrIS-TROPOMI data product.

Section 3.1.5 reviews the FTIR spectrometer retrievals and the NDACC network. Section 3.1.6 and 3.1.7 review the GEOS-Chem and GEOS-Chem Adjoint model characteristics, operation and application to the present study.

\subsubsection{MOPITT}

Measurement of Pollution in the Troposphere (MOPITT) is an atmospheric CO monitoring instrument and is one of 5 instruments aboard the Terra spacecraft (Drummond et al., 2010). The Terra satellite is part of National Aeronautics and Space Administration's (NASA) 
Earth Observing System (EOS) program for long-term environmental monitoring and was launched in December 1999 (Drummond et al., 2010). Terra has a Sun-synchronous polar low Earth orbit (LEO) at an altitude of $705 \mathrm{~km}$ (Drummond et al., 2010). Coverage is limited between $82^{\circ} \mathrm{S}$ and $82^{\circ} \mathrm{N}$, with $640 \mathrm{~km}$ wide swaths (Drummond et al., 2010). It has an equatorial local crossing time around 10:30 am (Thome, 2020) and circles Earth every 98 minutes, allowing MOPITT to provide near-global coverage of CO measurements in 3-4 days (Drummond et al., 2010).

The MOPITT instrument measures infrared signals at the top of the atmosphere, where CO measurements are isolated by using gas correlation radiometers (Francis et al., 2017). The MOPITT Algorithm Theoretical Basis Documents (ATBD) by Francis et al. (2017) describes the MOPITT instrument utilizing several moving parts, where $\mathrm{CO}$ is detected by measuring in nearinfrared (NIR) and thermal infrared (TIR) radiation channels at $2.3 \mu \mathrm{m}$ and $4.7 \mu \mathrm{m}$. The CO sensitivity of these signals is enhanced by passing the incoming radiation through pressure modulating cells (PMCs) and length modulating cells (LMCs) containing sample CO gas (Francis et al., 2017). By altering the gas pressure in the PMCs, MOPITT is able to increase sensitivity to low pressures in the upper troposphere (Francis et al., 2017). By altering the signal length in the LMCs, the instrument improves its sensitivity to the Earth's surface at higher pressures (Francis et al., 2017).

Francis et al. (2017) describes that the received infrared signals are duplicated on two optical tables, each with two scan mirrors and dichroic beam splitters which direct the signals to two length modulating cells (LMC) and one pressure modulating cell (PMC) each. In total there are 8 channels and 16 signals, which are passed to the detectors (Francis et al., 2017). For 
calibration, the scan mirrors turn from the Earth to face space to provide a cold calibration point, to an internal blackbody to provide a warm calibration point, and to a heated $460 \mathrm{~K}$ internal target to provide a hot calibration point (Francis et al., 2017). These calibration signals (Level 0 data) provide the radiometric offset and radiometer gain for thermal channels, which are then used to produce the Level 1 radiance data product (Francis et al., 2017). Further physical operation details and references can be found in the June 2017 ATBD (Francis et al., 2017).

To produce the Level 2 data product, the MOPITT ATBD explains that the Level 1 data is first filtered to remove data contaminated by clouds. Next, it passes through a retrieval algorithm to obtain the CO concentration values (Francis et al., 2017). A copy of the step-bystep processing flow chart from the MOPITT ATBD is available in the Appendix A.2. In the first step, MOPITT uses the Moderate Resolution Imaging Spectroradiometer (MODIS) instrument aboard Terra for cloud detection (Francis et al., 2017). MODIS measures visible and infrared bands which are then utilised in the MODIS cloud mask algorithm to determine if the surface is obstructed by clouds (Francis et al., 2017). The MODIS data is then co-located with MOPITT data and filtered through MOPITT cloud detection (Francis et al., 2017). MOPITT detects the presence of clouds by distinguishing the cold cloud thermal emissions compared to the warmer a priori clear-sky radiance calculated by the MOPITT Operational Fast Forward Model (MOPFAS) (Francis et al., 2017). Only acceptable clear-sky MOPITT Level 1 radiances are retained (Francis et al., 2017). These Level 1 clear sky data radiances are used to produce the Level 2 data product (Francis et al., 2017). This is achieved by using a retrieval algorithm, where the 
radiances are calculated using optimal estimation and a forward radiative transfer model to obtain CO vertical profiles (Francis et al., 2017).

Eqn. (10)

$$
y=F(x, b)+N_{\epsilon}
$$

where

$$
\begin{aligned}
& \boldsymbol{y}=\text { observed MOPITT radiances vector }(\text { Level } 1) \\
& \boldsymbol{F}=\text { forward radiative transfer model } \\
& \boldsymbol{x}=\text { state vector of desired CO retrieved variables } \\
& \boldsymbol{b}=\text { forward model parameters } \\
& \boldsymbol{N}_{\in}=\text { radiance error vector }
\end{aligned}
$$

As shown by Equation 10 (Francis et al., 2017), the forward radiative transfer model $F$ calculates radiances to match the observed MOPITT Level 1 radiances $(y)$. In this equation, $F$ is a function of $x$ and $b$, where $x$ are the CO state vectors and $b$ are other forward model parameters. The forward model parameters include a priori surface "skin temperature" and emissivity, temperature profiles, and water vapor profiles (Francis et al., 2017). For the more recent MOPITT Version 7, these meteorological profiles are calculated by Modern-Era Retrospective Analysis For Research And Applications Version 2 (MERRA-2) reanalysis (Francis et al., 2017).

As described in the MOPITT ATBD (Francis et al., 2017), a non-linear iterative maximum a posteriori (MAP) optimal estimation is used to iteratively find the model state vector $x$ that best matches the MOPITT observed radiances $y$. When the CO fractional change between iterations decreases to $5 \%$ or less, the desired $\mathrm{CO}$ state vector is found and is used as the CO 
profile (Francis et al., 2017). This MAP solution requires a priori state vectors, a priori covariance matrices, weighting function matrices, and radiance errors and error correlations (Francis et al., 2017). Francis et al. (2017) explains a priori state vectors "represent the geographical and seasonal variability of 'background' concentrations of $\mathrm{CO}$," and a priori covariance matrices "describe the expected variability and inter-level correlations of the CO profile." MOPITT Version 7 a priori state vectors are interpolated from the CAM-Chem model with $1^{\circ}$ latitude and longitude spatial resolution, monthly temporal resolution, and 2000-2009 climatology (Francis et al., 2017). This algorithm includes radiance bias correction for both the modeled radiances and Level 1 radiances (Francis et al., 2017). This incorporates instrumental, model, spectroscopy, and geophysical errors (Francis et al., 2017). The major error contributors being instrument noise, geophysical noise, and forward model errors (Francis et al., 2017). Radiative effects from aerosols are not included in the algorithm (Francis et al., 2017).

The MOPITT Version 7 Level 2 data product has a $22 \mathrm{~km} \times 22 \mathrm{~km}$ field of view (FOV) ground pixel resolution and CO concentrations are retrieved on a ten-level vertical pressure grid (Francis et al., 2017). The vertical grid has levels ranging from the surface to $100 \mathrm{hPa}$, where the retrieved values are the mean volume mixing ratio of each layer above the corresponding level (Deeter, MOPITT Algorithm Development Team, et al., 2017). The uppermost layer lies between $100 \mathrm{hPa}$ and $50 \mathrm{hPa}$, and fixed values above $50 \mathrm{hPa}$ are based on the CAM-chem model climatology(Deeter, MOPITT Algorithm Development Team, et al., 2017).

The NIR retrieval product performs best for daytime CO total column concentrations over land (Deeter, Edwards, et al., 2017) and has uniform sensitivity throughout the vertical extent of the troposphere (Deeter et al., 2013). The TIR retrieval product has similar 
performance during day and night over land and ocean (Deeter, Edwards, et al., 2017), and has higher CO sensitivity in the mid and upper-troposphere (Deeter et al., 2013). Since the NIR-TIR retrieval incorporates information from both channels, this product provides the best vertical resolution and enhanced lower-troposphere sensitivity; however, it also exhibits large random retrieval errors and bias drift (Deeter, Edwards, et al., 2017).

In this study, MOPITT Version 7 Level 2 combined NIR-TIR data products are used. These retrievals were obtained from the NASA Langley Research Center's (LaRC) Atmospheric Science Data Center (ASDC) Distributed Active Archive Center (DAAC) (Ziskin, 2019), using the NASA MOPITT search and subsetting web application. Validation of the NIR-TIR surface-level retrieval for MOPITT Level 2 version 7 against in-situ HIPPO field campaign data reveals a surface-level retrieval bias of $2.8 \%$, bias variability of $11 \%$, and correlation coefficient of 0.5 (Deeter, Edwards, et al., 2017). Although a correlation of 0.5 may seem low, it is a reasonable fit considering MOPITT retrievals are less sensitive at the surface and more heavily relies on the a priori (Deeter, Edwards, et al., 2017). MOPITT also provides a coarse vertical and horizonal resolution compared the in-situ aircraft measurements. The average profile correlation coefficient validated against five levels is also 0.5 , and the total column correlation coefficient is 0.98 (Deeter, Edwards, et al., 2017). Validation of the NIR-TIR retrieval against ground-based NDACC station data has a total column mean bias of 5.1\% (Buchholz et al., 2017).

An assessment of MOPITT Version 6 data explains how DOFS (or DFS as in the study) can be highly variable "geographically and temporally due to variability of instrument noise, geophysical noise, thermal contrast and CO loading" (Deeter et al., 2015). A low DOFS indicates less information about the shape of the vertical CO profile, DOFS can be considered a "measure 
of resolution" (Rodgers, 2000). For MOPITT, a DOFS of 1.0 or lower has poor profile information but is still useful for total column analysis (Deeter et al., 2015). Instrument noise reduces DOFS values, therefore indicating a reduction in profile shape information (Deeter et al., 2015). Deeter et al. (2015) determines that the DOFS reduction due to geophysical noise is noted to be an effect unique to MOPITT, which can be seen over mountainous regions. Thermal contrast increases DOFS values seasonally due to insolation, where it is expected to be highest in the summer and lowest in the winter (Deeter et al., 2015). Sensitivity of the NIR channel increases as CO concentrations increase, which in turn increases the DOFS (Deeter et al., 2015). They explain that it is expected that CO loading should be its highest in April and lowest in September within the Northern Hemisphere. For the NIR-TIR product their study found that the average DOFS values range from 1.0 to 2.0 over the Continental United States (CONUS), with a maximum in April except over Texas and Northern Mexico where the maximum is in July. Similarly, the average DOFS values range from 1.1 to 2.0 over the Amazon Basin, with a maximum in October near the end of the dry season (Deeter et al., 2015). A MOPITT Version 6 validation study against NDACC compares median DOFS values over a vertical range from the surface to 26 hPa for 14 NDACC stations between August 2001 and February 2012 (Buchholz et al., 2017). The MOPITT NIR-TIR values over NDACC stations range from 0.76 to 1.72 , with a mean of 1.32 over land and water (Buchholz et al., 2017).

The lifetime requirement for MOPITT was 5 years (Drummond et al., 2010) and was originally intended to measure both $\mathrm{CO}$ and $\mathrm{CH}_{4}$. Once MOPITT was in operation it was observed that $\mathrm{CH}_{4}$ retrievals were not precise enough to detect source and sink variations, and therefore $\mathrm{CH}_{4}$ products have not been produced (Francis et al., 2017). The ATBD by Francis et 
al. (2017) describes that in 2001, 4 channels ceased producing useful data due to a failed cooler and signal chopper. In 2009, a slow decrease in mean gas pressure in the PMC and pressure in the LMC were discovered, indicating a probable slow gas leak (Francis et al., 2017). Despite these operational setbacks, remote instrument adjustments and algorithm alterations have allowed MOPITT to still operate 21 years later (Francis et al., 2017).

Although MOPITT is still operating and is often used as a remote sensing CO standard product for comparisons, it will eventually need to be replaced before it ceases operation. The CrIS and TROPOMI satellite instruments are promising candidates to replace MOPITT. They provide, respectively, SWIR and TIR retrievals at greater spatial and temporal resolutions.

\subsubsection{CrIS}

The Cross-Track Infrared Sounder (CrIS) is an atmospheric monitoring instrument aboard the Suomi National Polar-orbiting Partnership (Suomi-NPP) satellite and NOAA-20 satellite (JPSS, n.d.). The CrIS instruments are part of the Joint Polar Satellite System (JPSS) program by the National Oceanic and Atmospheric Administration (NOAA) and NASA (JPSS, n.d.), and are used to generate Environmental Data Record (EDS) and Essential Climate Variables (ECV) products from the NOAA Unique Combined Atmospheric Processing System (NUCAPS) (NCEI, 2018) and Community Long-term Infrared Microwave Combined Atmospheric Product System (CLIMCAPS) (Smith \& Barnet, 2019) respectively. CrIS was built to continue and improve upon the heritage of satellite instrument measurements including Atmospheric InfraRed Sounder (AIRS) and Infrared Atmospheric Sounding Interferometer (IASI) (Gambacorta, 2013). 
The Suomi NPP (formerly named National Polar-orbiting Operational Environmental Satellite System NPOESS Preparatory Project, or NPP) was launched in October 2011 (JPSS, n.d.). The NOAA-20 (formerly named JPSS-1) was launched in November 2017 and follows the same orbit as Suomi-NPP, 50 minutes behind (JPSS, n.d.). At a higher orbit than TERRA, the Suomi-NPP and NOAA-20 can provide daily global coverage compared to the 3-4 day global coverage by MOPITT (Drummond et al., 2010). The Suomi NPP and NOAA-20 platforms have a Sun-synchronous near-polar orbit at an altitude of 824 km (Cao et al., 2018; D. Fu et al., 2016). CrIS's square fields of regards (FORs) make up the $2200 \mathrm{~km}$ swath, where each FOR contains a $3 \times 3$ set of field of views (FOVs) (Cao et al., 2018; D. Fu et al., 2016). The swath covers both day and night observations at 1:30 am/pm equatorial crossing times, providing near-global daily coverage (Cao et al., 2018; Gambacorta, 2013).

The NUCAPS algorithm was developed by the NOAA and National Environmental Satellite, Data, and Information Service (NESDIS) Center for Satellite Application and Research (STAR), and is produced by their Office of Satellite Product Operations (OSPO) (NCEI, 2018). The CLIMCAPS algorithm shares its heritage with the NUCAPS algorithm, and like NUCAPS it retrieves profiles of temperature, water vapour, greenhouse and pollutant gases (including CO), cloud information, and surface temperature and emissivity along with diagnostics (Smith \& Barnet, 2019).

CrIS uses a Fourier Transform Spectrometer (FTS) to measure 1305 spectral channels over three infrared (IR) bands simultaneously aboard the Suomi-NPP with varying nominalspectral-resolution (NSR): long-wave IR (LWIR) at $0.625 \mathrm{~cm}^{-1}$, mid-wave IR (MWIR) at $1.25 \mathrm{~cm}^{-1}$, and short-wave infrared (SWIR) at $2.5 \mathrm{~cm}^{-1}$ (Smith \& Barnet, 2020). Aboard the NOAA-20, "CrIS 
is in full-spectral-resolution (FSR) mode with all spectral bands sampled at $0.625 \mathrm{~cm}^{-1 \prime \prime}$ (Smith \& Barnet, 2020). The CrIS User's Guide by Cao et al. (2018) describes the physical components of the CrIS instrument, which includes a beamsplitter, a porchswing moving mirror, a stationary dynamic alignment mirror, a laser metrology system, and detectors. Here, incoming IR is divided by the beamsplitter and directed towards the moving mirror and the stationary mirror (Cao et al., 2018). These divided beams are then reflected back and recombined at the beamsplitter (Cao et al., 2018). The optical path difference changes as the moving mirror's position shifts, allowing the identification of the time-varying interference pattern, called an interferogram (Cao et al., 2018). This recombined light then passes through the telescope and dichroic splitters, where the radiance beam is split into three separate beams and read by the three detector focal planes for LWIR, MWIR and SWIR (Cao et al., 2018). For calibration, radiance is measured from the Internal Calibration Target (ICT), a high-precision calibration blackbody, and Deep Space (DS) (Cao et al., 2018). Instead of heating or cooling the internal calibration points to a constant temperature, their temperatures are monitored (Cao et al., 2018).

These interferogram measurements and calibration data are referred to as the Raw Data Records (RDRs) in the NUCAPS ATBD, which are then converted into the radiance data referred to as the Sensor Data Records (SDRs) (Cao et al., 2018). The NUCAPS algorithm uses SDR Cloud-Cleared Radiances to produce the Standard Product (Level 2 dataset) containing profile information (Gambacorta, 2013). This NUCAPS algorithm is described as six modules (Gambacorta, 2013). A copy of the processing flow chart from the NUCAPS ATBD is shown in Appendix A.3. The CLIMCAPS algorithm is described in 8 steps, using cloud cleared radiances 
and a Bayesian Optimal Estimation inversion framework to produce its Level 2 product (Smith \& Barnet, 2019). The Bayesian Optimal Estimation inversion allows for higher quality retrievals than NUCAPS as it "accounts for spectral interference from other state variables so that the correlation among ECVs (and their uncertainty) may be minimized" (Smith \& Barnet, 2019). This careful tracking of error and correlation information within the CLIMCAPS algorithm permits the application of these auxiliary data directly into data assimilation techniques such as Kalman filtering and variation assimilation. The CLIMCAPS ATBD is not currently obtainable, but an article by Smith \& Barnet (2019) is available describing important algorithm differences between CLIMCAPS and NUCAPS. A copy of the CLIMCAPS processing flow chart is shown in Figure 3.1.2.1 (Smith \& Barnet, 2019). 


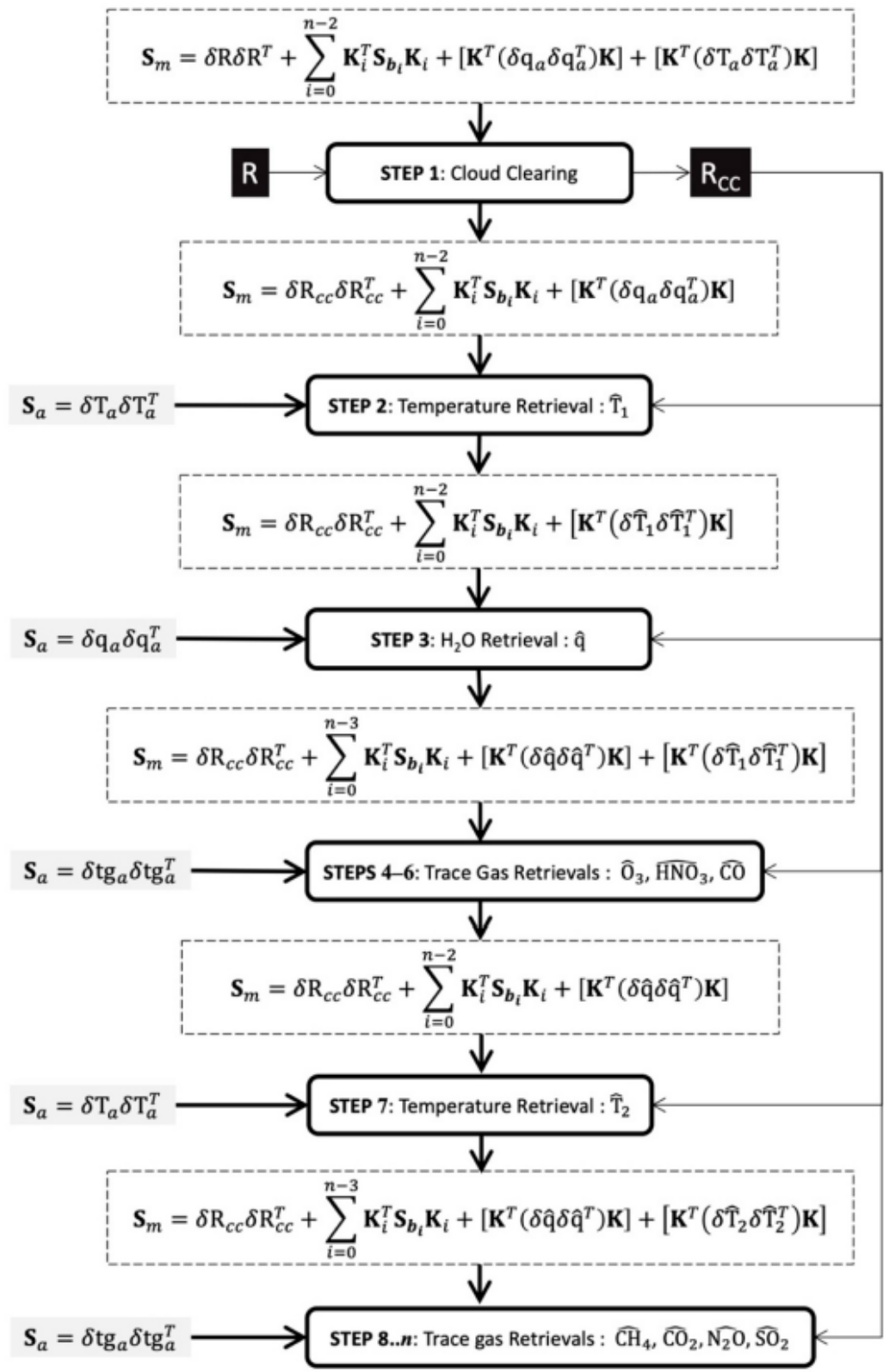

(Smith \& Barnet, 2019)

Figure 3.1.2.1: CrIS CLIMCAPS CO retrieval algorithm flow chart of processing Level 1 data to Level 2 data (Smith \& Barnet, 2019)

The first step in the CLIMCAPS algorithm generates "cloud cleared" radiances from the Level 1 radiance product (Smith \& Barnet, 2019). A “cloud cleared" radiance product is the calculated expected radiance if there were no clouds. This is obtained through using the same 
basic methodology as the NUCAPS algorithm. Between each FOV within a datum (Level 2 retrieval footprint), surface properties, cloud type, and atmospheric state is assumed to be uniform (Gambacorta, 2013; Smith \& Barnet, 2019). Wherein the only differences between FOVs are assumed to be from varying cloudiness known as the cloud fraction (Gambacorta, 2013; Smith \& Barnet, 2019). In the CLIMCAPS algorithm, a linear least squares approach is used to retrieve a cloud cleared radiance from the sets 3x3 FOVs (Smith \& Barnet, 2019).

As described in Smith \& Barnet (2019), steps 2 and 3 are where the first temperature retrieval and the water vapour retrieval are each calculated using a forward radiance model and the MERRA-2 reanalysis product as a priori first guess estimates. Here, the a priori error covariance matrix is determined from a MERRA-2 reanalysis and (European Centre for MediumRange Weather Forecasts) ECMWF interpolation to CrIS space-time resolution (Smith \& Barnet, 2019). These temperature and water vapour profiles are then used to calculate $\mathrm{O}_{3}, \mathrm{CO}$, and $\mathrm{HNO}_{3}$ profiles (Steps 3 to 6) (Smith \& Barnet, 2019). This is followed by a second temperature retrieval (Step 7) (Smith \& Barnet, 2019). The second temperature retrieval uses the MERRA-2 reanalysis product as the a priori again, but updates the uncertainty estimates for clouds and background variables (Smith \& Barnet, 2019). Finally, step 8 is where the remainder of the CLIMCAPS greenhouse and precursor gases are retrieved including $\mathrm{CH}_{4}, \mathrm{CO}_{2}, \mathrm{~N}_{2} \mathrm{O}$, and $\mathrm{SO}_{2}$ (Smith \& Barnet, 2019). It should be noted that Smith \& Barnet (2019) specify "CLIMCAPS evaluates the information content of each datum individually and does not make a priori assumptions about the type or amount of information a measurement should contain."

For this study, CrIS Level 2 CO concentrations were obtained from the CLIMCAPS Version 2 product series (SNDRJ1IML2CCPRET) (Barnet, 2019). CLIMCAPS data is available for both 
Suomi-NPP and NOAA-20 CrIS instruments and is publicly accessible from the Goddard Earth Sciences Data and Information Services Center (GES DISC) Earth Observing System Data and Information System (EOSDIS) EARTHDATA website (Berrick, 2020). The CLIMCAPS CO data product provides a $14 \mathrm{~km}$ diameter FOV, and a vertical profile consisting of 100 layers ranging from $1100 \mathrm{hPa}$ to $0.016 \mathrm{hPa}$ (Barnet, 2019). CrIS's CO TIR retrieval product is sensitive to CO in the mid- and upper-troposphere (like MOPITT), with a typical signal-to-noise ratio of $800: 1$ (D. Fu et al., 2016). The CLIMCAPS' DOFS varies daily and seasonally like its retrievals and is typically below 1.5 , indicating a "sensitivity to the true state of $\mathrm{CO}$ at a target scene in one vertical layer" (Smith et al., 2020b). A DOFS below 0.5 would indicate a "very low observing capability of CO" and "contains mostly a-priori information and measurement uncertainty", which is seen at the South Pole (Smith et al., 2020b). In another study, CrIS DOFS is compared from 27 to 28 August 2013 both in the lowermost troposphere (LMT) and free troposphere (D. Fu et al., 2016). Here, the DOFS in the lower-most troposphere (surface to $3 \mathrm{~km}$ or $700 \mathrm{hPa}$ ) is low at 0.62 compared to 0.94 for $3 \mathrm{~km}$ to TOA (D. Fu et al., 2016). Combined, the DOFS is 1.57 (D. Fu et al., 2016).

Likely due to CrIS's large dataset sizes and its more recent availability, there are not many published works comparing accuracy, precision, and bias with ground station networks. However, preliminary analysis comparing NUCAPS and CLIMCAPS, and CrIS CO with other instruments show promising results (Smith \& Barnet, 2019; Warner, 2020). The high vertical and temporal resolution, large swath width providing near-global daily coverage, and cloud clearing algorithm makes CrIS an excellent candidate for improving on and continuing MOPITT CO profile retrieval. 


\subsubsection{TROPOMI}

The TROPOspheric Monitoring Instrument (TROPOMI) is an atmospheric monitoring instrument aboard the Copernicus Sentinel 5 Precursor (S5P) satellite was launched in April 2018 (KNMI R\&D Satellite Observations, n.d.). TROPOMI has an expected lifetime of seven years and was built to continue and improve upon the heritage of satellite instrument measurements starting with Global Ozone Monitoring Experiment (GOME), Ozone Monitoring Instrument (OMI), and Scanning Imaging Absorption Spectrometer for Atmospheric Cartography (SCIAMACHY) (Babić et al., 2017). At an altitude of 817 km (Babić et al., 2017), the S5P follows 5 minutes behind Suomi NPP in a Sun-synchronous near-polar orbit, has a swath width of 2600 $\mathrm{km}$ and provides daily global coverage (D. Fu et al., 2016). It provides both day and night observations over a 17 day repeat cycle, where daytime observations are in the early afternoon (equatorial crossing time in the ascending note of 13:30) when the boundary layer is well developed and representative of pollution buildup (Babić et al., 2017). This high spatial resolution also allows for more cloud-free observations (Babić et al., 2017).

The ATBD for the TROPOMI L01b data processor by Babić et al (2017) describes the instrument's components and operation as follows. The TROPOMI instrument consists of four separate spectrometers that together measure wavelengths between ultraviolet and shortwavelength infrared (SWIR), enabling it to detect trace gases including nitrogen dioxide $\left(\mathrm{NO}_{2}\right)$, $\mathrm{O}_{3}, \mathrm{CH}_{4}, \mathrm{CO}$, sulphur dioxide $\left(\mathrm{SO}_{2}\right)$ and formaldehyde $\left(\mathrm{CH}_{2} \mathrm{O}\right)$, as well as information relating to clouds, aerosols and surface reflectance (Babić et al., 2017; Langerock et al., 2018). TROPOMI detects CO using its SWIR spectrometer between 2305 and 2385 nm (Babić et al., 2017), with a spectral resolution of about $0.25 \mathrm{~nm}$ (van Hees et al., 2018). The radiation entering the 
telescope is manipulated by multiple mirrors, prisms, and lenses to project a focused perpendicular beam perpendicular onto the two SWIR detectors (Babić et al., 2017). The calibration unit consists of a diffuser carousel and folding mirror (Babić et al., 2017). These allow for calibration with the Sun, an on-board white light source, a common LED, a spectral line source, and a dark measurement when light sources are blocked and turned off (Babić et al., 2017). This calibration data is used to calculate the Level 1 radiance data products, which contains Earth radiance, solar irradiance, calibration and background measurements, and engineering data (Babić et al., 2017).

The ATBD for TROPOMI's carbon monoxide total column retrieval by Landgraf et al. (2018) describes the retrieval process for the Level 2 products. They are calculated using a modified SWIR CO retrieval (SICOR) algorithm, based on the CO algorithm for the SCIAMACHY satellite instrument (Landgraf et al., 2018). A copy of the processing flow chart from the Landgraf et al. (2018) ATBD is shown in Figure 3.1.3.1. SICOR is a two-step retrieval algorithm where radiative transfer models are used like in the MOPITT algorithm. However, the method of optimal estimation and other calculating parameters are different (Landgraf et al., 2018). In SICOR, the model state vector is found iteratively (instead of MAP) to find the best match to the TROPOMI CO SWIR observed radiances (Landgraf et al., 2018). 


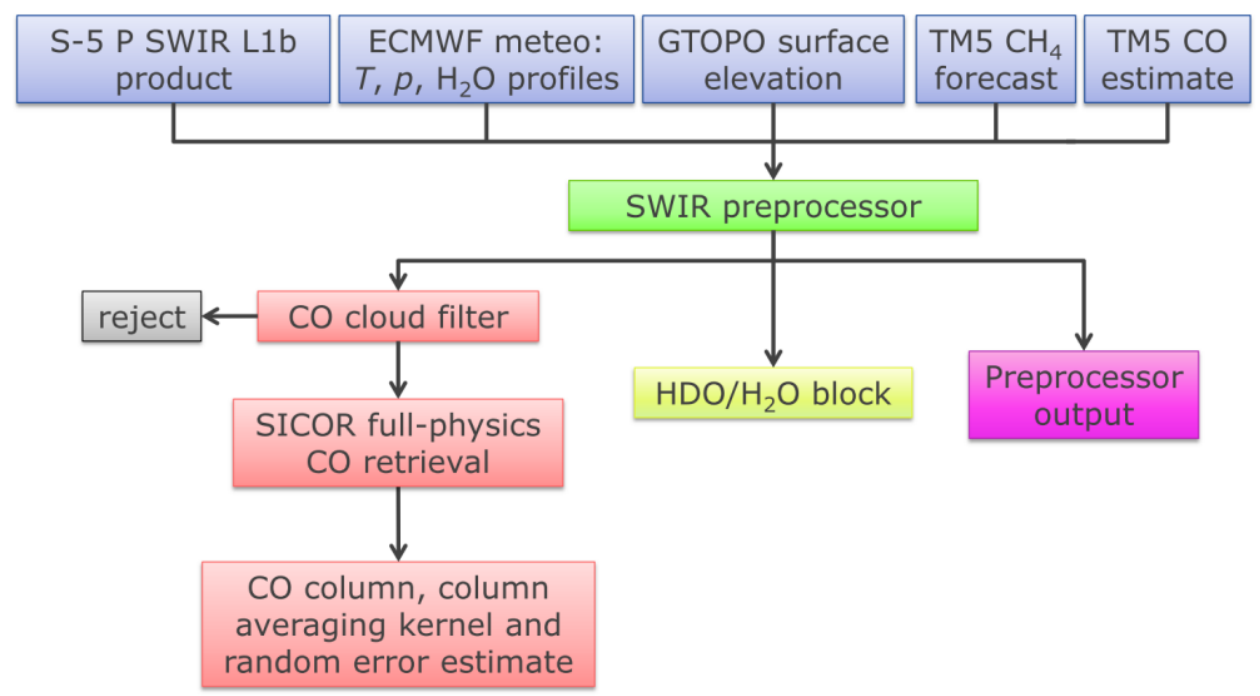

(Landgraf et al., 2018)

Figure 3.1.3.1: TROPOMI CO retrieval algorithm flow chart of processing Level 1 data to Level 2 data (Landgraf et al., 2018)

Landgraf et al. (2018) describes the first step, which uses a non-scattering radiative transfer model to determine the $\mathrm{CH}_{4}$ column, which in turn is used to detect and filter out data affected by optically thick water clouds. If the expected $\mathrm{CH}_{4}$ column is too low, it indicates cloud presence through obstruction (Landgraf et al., 2018). The total columns of interfering species in the $\mathrm{CO}$ band $\left(\mathrm{CH}_{4}, \mathrm{H}_{2} \mathrm{O}\right.$, and semi-heavy water ( $\left.\left.\mathrm{HDO}\right)\right)$, are then fit together with surface albedo and spectral offset to determine CO (Landgraf et al., 2018). The inversion is then calculated using the total column and $\mathrm{CH}_{4}$ a priori from a dedicated TM5 atmospheric transport model. Any data with a non-scattering $\mathrm{CH}_{4}$ error $\Delta \mathrm{CH}_{4}$ greater than $25 \%$ are assumed to have optically thick clouds, and are therefore removed (Landgraf et al., 2018).

The second step described in Landgraf et al. (2018) uses a scattering radiative transfer model along with the $\mathrm{CH}_{4}$ a priori knowledge, $\mathrm{CO}$ abundance, and $\mathrm{H}_{2} \mathrm{O}$ abundance to define effective cloud parameters. Here, the model inversion then determines the CO column using a 
least square fitting of the first guess CO reference profile generated by TM5 (Landgraf et al., 2018). Other dynamic input parameters such as temperature profile, specific humidity profile, surface pressure and elevation are sourced from the European Centre for Medium-Range Weather Forecasts (ECMWF) (Landgraf et al., 2018). Finally, the SICOR algorithm outputs a Level 2 data product containing the geospatially-tagged $\mathrm{CO}$ total column, column averaging kernels, and statistical noise estimates (Landgraf et al., 2018).

The first TROPOMI data products were publicly released in July 2018 (KNMI R\&D Satellite Observations, n.d.). In this study, TROPOMI data (Copernicus Sentinel-5P (processed by ESA), 2018) was retrieved from the Copernicus Sentinel-5P Open Access Hub, which is publicly available at https://scihub.copernicus.eu/. TROPOMI provides a $7 \mathrm{~km} \times 7 \mathrm{~km}$ FOV and retrieves $\mathrm{CO}, \mathrm{H}_{2} \mathrm{O}, \mathrm{HDO}$, and $\mathrm{CH}_{4}$ total columns with supporting information (Langerock et al., 2018). TROPOMI's SWIR retrieval product is sensitive to CO total column, with a minimum spectral signal-to-noise ratio of $120: 1$ (D. Fu et al., 2016). Preliminary validation against NDACC and Total Carbon Column Observing Network (TCCON) ground-based stations reveal a bias of the order of $10 \%$ and a random uncertainty median of approximately $7 \%$ for data between November 2017 and May 2018 (Langerock et al., 2018). Since 6 August 2019, TROPOMI data products have an updated to provide a $7.2 \mathrm{~km} \times 5.6 \mathrm{~km}$ FOV (KNMI R\&D Satellite Observations, n.d.). The effective DOFS of the TROPOMI product is 1.0 as it has uniform sensitivity over the column (Borsdorff et al., 2014, 2018). In Fu et al. (2016), TROPOMI DOFS is also compared from 27 to 28 August 2013 in the LMT and free troposphere. Here, the DOFS difference between them is smaller than CrIS's at 0.61 for the LMT, 0.71 for $3 \mathrm{~km}$ to TOA, and 1.32 combined (Fu et al., 2016). 
Only total column values were available for this study, as profile information is not provided in the data product. TROPOMI's spatial and temporal resolution, large swath width providing near-global daily coverage, and cloud clearing algorithm make TROPOMI an excellent candidate for improving on and continuing the MOPITT CO total column retrieval.

\subsubsection{Joint CrIS-TROPOMI}

As discussed in sections above CrIS and TROPOMI observe and retrieve different atmospheric $\mathrm{CO}$ information. CrIS CO retrievals are most sensitive to altitudes above $700 \mathrm{hPa}$ (Smith et al., 2020b), and TROPOMI CO retrievals are sensitive to the total column (Landgraf et al., 2018). Individually these observation windows are similar to the separate MOPITT NIR and TIR products and have a potential to provide greater $\mathrm{CO}$ profile information if combined into a NIR-TIR product. Fu et. al. (2016) investigates the potential of a Joint CrIS-TROPOMI product, wherein such a product could provide high spatial resolution profiles with higher temporal sampling in place of MOPITT when it retires.

A multispectral retrieval combining radiances from the CrIS and TROPOMI satellite instruments is being developed by NASA's Jet Propulsion Laboratory (JPL) using the MultiSpEctra, Multi-SpEcies, Multi-Sensors (MUSES) retrieval algorithm (D. Fu et al., 2016). Although this product is not yet available, D. Fu et al. (2016) notes it should provide an improved temporal, spatial, and vertical resolution measurements of tropospheric CO compared to MOPITT. They describe that the daily global coverage will have, $14 \mathrm{~km} \times 14 \mathrm{~km}$ ground pixels, an approximate $15 \%$ error reduction in the lowermost troposphere, and spectral signal-to-noise ratio greater than $240: 1$. Their study compares the DOFS of CrIS, TROPOMI, and MOPITT from 27 to 28 August 2013 in the LMT and from $3 \mathrm{~km}$ to TOA. In both the LMT and $3 \mathrm{~km}$ to TOA 
section, both CrIS and TROPOMI have higher individual DOFS values than the respective individual NIR and TIR products from MOPITT (D. Fu et al., 2016). Consequently, the synergistic Joint CrIS-TROPOMI retrieval has a higher DOFS than the MOPITT NIR-TIR product where the DOFS from the surface to TOA is on average 2.22 compared to 1.88 respectively (D. Fu et al., 2016).

While this joint product is not yet available, its use may enhance this study's investigation and should be considered for future study. The integration of TROPOMI's high surface sensitivity can augment CrIS's boundary layer profile $\mathrm{CO}$. These instruments provide and enhanced ground resolution compared to MOPITT, and view the same location on the planet at a higher frequency. For example, MOPITT's $22 \mathrm{~km} \times 22 \mathrm{~km}$ FOV and 2-3 day global coverage (Francis et al., 2017) versus CrIS and TROPOMI's 14 km FOV and daily global coverage (D. Fu et al., 2016; Smith \& Barnet, 2020). A higher resolution Joint CrIS-TROPOMI product may also in turn provide higher resolution $\mathrm{OH}$ distributions when assimilated into the GEOS-Chem model.

\subsubsection{NDACC}

The Network for the Detection of Atmospheric Composition Change (NDACC) is an international network consisting of over 90 ground-based atmospheric research stations around the globe (De Mazière et al., 2018). De Mazière et al. (2018) states that NDACC's formation stemmed from growing concern over stratospheric ozone in the 1970s, and the mandated long-term monitoring of the ozone layer in 1985 by the International Vienna Convention for the Protection of the Ozone Layer. With input from NASA, NOAA, and the Chemical Manufacturers Association (CMA), the network began operation in January 1991 as 
the Network for Detection of Stratospheric Change (NDSC) (De Mazière et al., 2018). Since that time, the network has expanded to also observe gases of relevance for tropospheric pollution like $\mathrm{CO}$ and $\mathrm{CH}_{4}$ (De Mazière et al., 2018). The NDACC network monitors numerous atmospheric constituents up to the lower mesosphere with a variety of ground-based measuring instruments (De Mazière et al., 2018). Its current objectives include establishing long-term databases for detecting trends, filling knowledge gaps, and evaluation of atmospheric models and satellite observations (De Mazière et al., 2018).

Today there are 20 stations equipped with active Fourier-Transform Infrared (FTIR) spectrometers that are able to monitor CO concentration profiles (NDACC, 2021). The NDACC FTIR instruments commonly used are the Bruker IFS series (125 HDR, 120 HR, 125 HR, and 120 M), and less commonly used is the Bomem DA8 (NDACC, 2021). These spectrometers use scanning Michelson interferometers to measure solar radiation, which requires clear sky (or thin cloud) conditions (Batchelor et al., 2009), and are paired with a solar tracker to obtain the solar zenith angle (SZA) (Zhou et al., 2018). The Bruker 125 HR contains two beam splitters (one for NIR and one for mid-IR) and two liquid nitrogen cooled detectors (one photovoltaic and one photoconductive) for different wavelengths (Batchelor et al., 2009). These detectors typically utilize standard NDACC narrow-band filters to "limit the wavenumber range of the spectra, thus increasing the signal-to-noise ratio" (Batchelor et al., 2009).

The CO retrieval windows used are 2057.70 to $2058.00,2069.56$ to 2069.76 , and 2157.50 to $2159.15 \mathrm{~cm}^{-1}$ with a spectral resolution between 0.0035 to $0.0070 \mathrm{~cm}^{-1}$ in the mid-IR (MIR) (Zhou et al., 2019). These wavelengths contain interference from $\mathrm{O}_{3}, \mathrm{CO}_{2}$, carbonyl sulfide (OCS), $\mathrm{N}_{2} \mathrm{O}$ and $\mathrm{H}_{2} \mathrm{O}$ (Zhou et al., 2019). To retrieve the $\mathrm{CO}$ profiles from the solar signals, 
NDACC employs one of two retrieval codes: SFIT2 or PROFFIT9 (Hase et al., 2004; Kerzenmacher et al., 2012; Zhou et al., 2019). SFIT2 was developed by C. Rinsland of NASA Langley and B. Connor of the National Institute of Water and Atmospheric Research (NIWA) in Lauder, New Zealand, and the PROFFIT9 algorithm was developed by Hase (Hase et al., 2004). Both retrieval codes use Rodgers optimal estimation (Rodgers, 2000), are in "excellent agreement", and have consistent resolution kernel matrices (Hase et al., 2004). Differences between them include the precise fitting method and signal-to-noise weighting. SFIT2 fits multiple spectral intervals simultaneously and an assumed percentage variability of the a priori profile for each layer, whereas PROFFIT9 conducts its fitting by scaling volume mixing ratio (VMR) profiles (Hase et al., 2004). SFIT2 weights its solutions according to an assumed signal-to-noise ratio, whereas PROFFIT9 does not assume a signal-to-noise ratio and instead uses residuals of the fit (Hase et al., 2004).

The final CO NDACC dataset contains total column and vertical profile information consisting of 47 points ranging up to an altitude boundary of $120 \mathrm{~km}$ (NDACC, 2021). These retrievals are most sensitive to concentrations from the surface up to approximately 20 kilometres (Langerock et al., 2018). A fixed NDACC CO a priori profile is derived from the Whole Atmosphere Community Climate Model (WACCM) version 4 (Zhou et al., 2018). Bruker instrument systematic uncertainty is mainly due to variations in temperature and uncertainty in spectroscopic parameters, and its random uncertainty mainly originates from atmospheric temperatures and the SZA (Zhou et al., 2019).

The data used in this research were obtained from several NDACC locations maintained by various representatives and organizations (see Appendix A.1 for full list) and are publicly 
available (see http://www.ndacc.org). Systematic smoothing error from spectroscopic parameters and temperature profiles is between 0.1 and $0.8 \%$ (Zhou et al., 2019). The random smoothing error from the solar zenith angle and temperature is approximately $0.3 \%$ (Zhou et al., 2019). Zhou et al. also infer that NDACC stations have a Northern Hemispheric bias when compared with the FTIR spectrometers of the Total Carbon Column Observing Network (TCCON). A MOPITT Version 6 validation study against NDACC compares median DOFS values over a vertical range from the surface to $26 \mathrm{hPa}$ for 14 NDACC stations between August 2001 and February 2012 (Buchholz et al., 2017). The NDACC values for the FTS instruments range from 1.65 to 2.92, with a mean of 2.22 (Buchholz et al., 2017).

NDACC datasets are readily available and can be accessed by the public. This highquality tropospheric data and provides a suitable basis for $\mathrm{CO}$ satellite evaluation in the troposphere (eg. Buchholz et al., 2017; Kerzenmacher et al., 2012). In this study, 2016 NDACC CO retrievals are compared against retrievals from MOPITT, CrIS, and TROPOMI with GEOSChem model outputs used as a common intercomparison platform. Note that 2019 datasets were not available until recently, and therefore comparisons with CrIS and TROPOMI 2019 retrievals are not direct.

\subsubsection{GEOS-Chem}

GEOS-Chem is an open-access 3-D atmospheric chemical transport model (CTM) with meteorological input from the Goddard Earth Observing System (GEOS) of the NASA Global Modeling and Assimilation Office (GMAO) (GEOS-Chem Support Team, 2021). The GEOS-Chem website prepared by the GEOS-Chem Support Team (2021) describes three different configurations of the model: GEOS-Chem Classic (GCC), GEOS-Chem High Performance (GCHP), 
and GEOS-Chem in on-line applications. GEOS-Chem also has two data archives: the operational data stream GEOS-Forward Processing (GEOS-FP) and the MERRA-2 reanalysis. The model is developed and used by research groups worldwide, is directed by the GEOS-Chem Steering Committee and User Working Groups, and "is managed by the GEOS-Chem Support Team, based out of Harvard University and Dalhousie University who manage the model with support from the US NASA Earth Science Division, the Canadian National and Engineering Research Council, and the Nanjing University of Information Sciences and Technology" (GEOS-Chem Support Team, 2021).

Bey et al. (2001) provided the first detailed GEOS-Chem model description (GEOS-Chem Support Team, 2021) and assessed the model performance with 1994 atmospheric observations. Bey et al. describes the goal of the model's development was to better understand tropospheric ozone chemistry, focusing on ozone-NOx-hydrocarbon relationships. To enable this study, the model utilises: known atmospheric chemistry processes; assimilated meteorological data; emission data from models and inventories of anthropogenic, biomass burning, and biogenic sources; photolysis and mass balance algorithms; and a simplified stratosphere to serve as a production/loss gas exchange mechanism across the tropopause.

Bey et al. (2001) analysed the GEOS-Chem v3-02 model output with 1994 tropospheric observations and concluded GEOS-Chem can reasonably represent global distributions of tropospheric $\mathrm{O}_{3}, \mathrm{NO}$, peroxyacetylnitrate (PAN) and other related species. Notable discrepancies included a possible overestimation of $\mathrm{OH}$ (up to 20\%), systematic underestimation of $\mathrm{CO}$, underestimation of ethane, underestimation of acetone, and overestimation of $\mathrm{HNO}_{3}$ in the remote troposphere (Bey et al., 2001). Underestimations are 
likely associated with underestimations of sources, and in the case of $\mathrm{CO}$ the possible overestimation of $\mathrm{OH}$ (Bey et al., 2001). Since v3-02, many chemical and transportation parameters have been updated and updated including emission inventories, chemical mechanisms, cloud modeling etc. (GEOS-Chem Support Team et al., 2021). The GEOS-Chem model continues to evolve as atmospheric science continues to be researched.

The most recent GEOS-Chem version is v12.9.3 released on 6 August 2020 (GEOS-Chem Support Team et al., 2021). This research utilises GCC version v8-02-01 with the GEOS5 data archive to simulate global trace gas concentrations, first released in May 2009 (GEOS-Chem Support Team et al., 2021). GCC v8-02-01 was selected for this research as this is the latest model version that can incorporate the GEOS-Chem Adjoint v35m (Adjoint Model and Data Assimilation Working Group, 2020). The adjoint development lags behind the main GEOS-Chem development by years, but does incorporate forward model updates from GEOS-Chem v9 and v10 (Adjoint Model and Data Assimilation Working Group, 2020). GCC calculates horizontal and vertical transport on a rectilinear latitude-longitude grid, with a spatial resolution of $2^{\circ} \times 2.5^{\circ}$ in the horizontal and with 47 vertical layers (GEOS-Chem Support Team et al., 2021).

Notable updates affecting $\mathrm{OH}$ and $\mathrm{CO}$ model since version v8-02-01 include emission updates (e.g. biogenic VOCs, lighting NOx, and anthropogenic emissions), chemistry updates (e.g. $\mathrm{O}_{3}$, isoprene, and PAN chemistry), and deposition updates (ozone deposition to the ocean, and wet deposition parameterization) (GEOS-Chem Support Team et al., 2021). These model changes can have a significant impact on modeled $\mathrm{OH}$ fields, and are tracked on the GEOSChem Wiki website (GEOS-Chem Support Team et al., 2021). 
Comparing benchmark v8-02-01 and v12-8-0 model runs, the airmass weighted $[\mathrm{OH}]_{\mathrm{GM}}$ changed from $11.812 \times 10^{5}$ to $10.613 \times 10^{5}$ molecules $\mathrm{cm}^{-3}$ (GEOS-Chem Support Team et al., 2021). However, it is noted that these model runs are for year 2005 and 2016 respectively and are not direct comparisons (GEOS-Chem Support Team et al., 2021). They are also assumed to be annual $[\mathrm{OH}]_{\mathrm{GM}}$ averages. In this study, before assimilation of CO retrievals, the base GEOSChem v8-02-01 airmass weighted $[\mathrm{OH}]_{\mathrm{GM}}$ ranges from $12.373 \times 10^{5}$ to $13.631 \times 10^{5}$ between May and August 2016. This is larger than both v8-02-01 and v12-8-0 [OH $]_{\mathrm{GM}}$ benchmark simulations. However, assuming a decadal IAV from Turner et. al. (2018) (Table 2.3.1.1.), the 2005 v8-02-01 mean of $11.812 \times 10^{5}$ is within close range of this study's base $12.373 \times 10^{5}$ for May 2016. It is also reasonable that this study's $[\mathrm{OH}]_{\mathrm{GM}}$ is larger than the $2005 \mathrm{v} 8-02-01$ mean since this study only considers months May through August, when $[\mathrm{OH}]_{\mathrm{GM}}$ is expected to be larger.

\subsubsection{GEOS-Chem Adjoint}

In this research, the GEOS-Chem Adjoint version v35m is used. This product is maintained by the GEOS-Chem community under Adjoint Model Scientist Daven Henze (GEOSChem Support Team, 2021). As described by Henze et al. (2007), "adjoint modeling is often employed as a method for determining the sensitivity of model predictions to input parameters and for optimizing these parameters to enforce agreement between the model predictions and an observational data set." In other words, an adjoint model is a tool that can be used to determine the sensitivity of one model variable to perturbations in other variables (Errico, 1997). It can be used in data assimilation, parameter estimation, stability analysis, and synoptic studies (Errico, 1997). This research uses the adjoint model to determine the sensitivity of CO as 
seen by each observing platform to $\mathrm{CO}$ emissions and to the chemical reaction rate of $\mathrm{OH}$ with $\mathrm{CH}_{4}$. It is also used to assimilate $\mathrm{CO}$ observations into the $\mathrm{GCC}$ model through several iterations to obtain a more accurate global $\mathrm{OH}$ field.

Generally, model forecast $U_{n}$, where $n$ are the variables of interest) sensitivity is evaluated against variability in their model outputs $(\boldsymbol{b})$ (Errico, 1997). This relationship is shown in Equation 11 (Errico, 1997), where $J^{\prime}$ denotes perturbations in the forecast of a select $J_{n}$. However, these forecasts can also be evaluated with their sensitivity to model inputs $(\boldsymbol{a})$. This relationship is shown in Equation 12 (Errico, 1997), where $J^{\prime}{ }_{n}$ denotes the perturbations of $J_{n}$. Errico (1997) describes the gradients $\partial J / \partial \boldsymbol{b}$ and $\partial J / \partial \boldsymbol{a}$ as the sensitivities to the model outputs and inputs respectively. The adjoint model is a tool that can efficiently determine the elements of the matrices $\partial J / \partial \boldsymbol{b}$ and $\partial J / \partial \boldsymbol{a}($ Errico, 1997). There are limitations to adjoint modeling as it is only useful when the above relationships are close to linear such as in the case of short time frames (Errico, 1997). The further back in time the model needs to calculate, the larger and more non-linear perturbations become, which therefore negatively impacts the accuracy of the adjoint model (Errico, 1997).

Eqn. (11)

$$
J^{\prime}=\sum_{k} \frac{\partial J_{n}}{\partial b_{k}} \Delta b_{k}
$$

Eqn. (12)

$$
J_{n}^{\prime}=\sum_{k} \frac{\partial J_{n}}{\partial a_{k}} \Delta a_{k}
$$


where $\quad J^{\prime}{ }_{n}=$ perturbations in model forecast for $n$ variable

$$
\begin{aligned}
& J^{\prime}=\text { perturbations in model forecast for one variable } \\
& a_{k}=\text { spatial and temoral input for } k \text { parameters } \\
& b_{k}=\text { spatial and temporal output for } k \text { parameters }
\end{aligned}
$$

The GEOS-Chem adjoint operates by first modeling a projected forecast forward in time with respect to perturbations in the model output as seen in Equation 11 (Errico, 1997). The adjoint model operator then relates the model output perturbations to perturbations of the model input through a first-order Taylor series approximation, seen in Equation 13 (Errico, 1997). Errico (1997) notes that this approximation only works for where the input perturbations are "sufficiently small" and where the calculation of model derivatives in Equation 13 is possible. To increase computational efficiency, the model is considered as a sequence of operations to determine the net result of Equation 13 (Errico, 1997). Equation 14 represents this sequence, where subscripts are "individual time steps, iterations, and physical or algorithmic components of the model" (Errico, 1997). The chain rule derivative of Equation 14 is then used to produce Equations 15 to 17, the sequential version of Equation 13 (Errico, 1997).

Eqn. (13)

$$
\Delta b_{j} \approx b_{j}^{\prime}=\sum_{k} \frac{\partial b_{j}}{\partial a_{k}} a_{k}^{\prime}
$$

Eqn. (14)

$$
B(\boldsymbol{a})=B_{N}\left(\left(\ldots\left\langle B_{2}\left\{B_{1}\left[B_{0}(\boldsymbol{a})\right]\right\}\right\rangle \ldots\right)\right)
$$


Eqn. (15)

$$
b_{j}^{\prime}=b_{j}^{\prime(N)}
$$

Eqn. (16)

$$
b_{j}^{\prime}=\sum_{k} \frac{\partial{b_{j}^{\prime}}_{j}^{(n)}}{\partial{b_{j}^{\prime}}_{j}^{(n-1)}} b_{j}^{\prime(n-1)}, \text { for } N \geq n \geq 1
$$

Eqn. (17)

$$
{b^{\prime}}_{j}^{(0)}=\sum_{k} \frac{\partial b_{j}^{(0)}}{\partial a_{k}} a_{k}^{\prime}
$$

where $\quad B=$ model operator

$$
b_{j}^{(n)}=j^{\text {th }} \text { component of the output after step } n
$$

The derivatives of Equation 13 (or Equation 15 to 17) are described in a matrix, known as the Jacobian matrix, and describes output perturbations with regards to model input parameters (Errico, 1997). To determine model sensitivity, the relationship must also include the model forecast (Errico, 1997). Equation 18 (Errico, 1997) shows this relationship, where the input sensitivity is determined by the output parameters. Here, note that the $j$ and $k$ are switched for the inputs and outputs compared to Equation 13. This indicates that a transpose of the Jacobian matrix is used, known as the adjoint (Errico, 1997).

Eqn. (18)

$$
\frac{\partial J}{\partial a_{j}}=\sum_{k} \frac{\partial b_{k}}{\partial a_{j}} \frac{\partial J}{\partial b_{k}}
$$


For the assimilation of observations, the adjoint model runs through forward and backward iterations over a specified time window. Retrievals from an observing instrument (e.g. CO retrievals from MOPITT) are integrated into the model as the model compensates to match the observed retrievals. The sensitivity of the model to these inputs becomes the cost function as in Equation 19 (Hakami et al., 2005). In a data assimilation context, the cost function $(J)$ may be large at first, but each iteration further optimises the model inputs using the calculated gradients to eventually find the minimum cost function (Henze et al., 2007). Once the minimum cost function is determined (plateaus) then assimilation of the observations into the models is optimised and complete.

$$
\begin{aligned}
& \text { Eqn. (19) } J=\frac{1}{2} \sum_{\boldsymbol{c} \in \Omega}\left(\boldsymbol{c}-\boldsymbol{c}_{o b s}\right)^{T} \boldsymbol{S}_{o b s}^{-1}\left(\boldsymbol{c}-\boldsymbol{c}_{o b s}\right) \\
& +\frac{1}{2} \gamma_{r}\left(\boldsymbol{p}-\boldsymbol{p}_{a}\right)^{T} \boldsymbol{S}_{p}^{-1}\left(\boldsymbol{p}-\boldsymbol{p}_{a}\right) \\
& \text { where } \quad J=\text { cost function } \\
& \Omega=\text { temporal and spatial domain of model and } \\
& \text { observation variables } \\
& \boldsymbol{c}=\text { vector of model species concentrations mapped to } \\
& \text { observation space } \\
& \boldsymbol{c}_{\text {obs }}=\text { vector of species observations } \\
& \boldsymbol{S}_{\text {obs }}=\text { observation error covariance matrix } \\
& \gamma_{r}=\text { regularisation parameter } \\
& \boldsymbol{p}=\text { vector of active model parameters throughout the model domain }
\end{aligned}
$$




$$
\begin{aligned}
& \boldsymbol{p}_{a}=\text { initial estimate of } \boldsymbol{p} \\
& \boldsymbol{S}_{p}=\text { error covariance estimate of } \boldsymbol{p} \text { and } \boldsymbol{p}_{a}
\end{aligned}
$$

As shown in Equation 19 (Henze et al., 2007), the cost function relies on the differences between the model and observational species vectors ( $\boldsymbol{c}$ and $\boldsymbol{c}_{\text {obs }}$ ), initial and active parameters $\left(\boldsymbol{p}\right.$ and $\left.\boldsymbol{p}_{a}\right)$, and corresponding covariance estimates $\left(\boldsymbol{S}_{o b s}\right.$ and $\left.\boldsymbol{S}_{p}\right)$, balanced by a regularisation parameter $\left(\gamma_{r}\right)$ (Henze et al., 2007). The regularisation parameter is a weighting factor, balancing the weight of the input observations (first term in Equation 19) versus the background parameters (second term) (Hakami et al., 2005). The covariance matrices $\boldsymbol{S}_{\text {obs }}$ and $\boldsymbol{S}_{p}$ describe the vector uncertainty, where $\boldsymbol{S}_{o b s}$ comes from the satellite product and $\boldsymbol{S}_{p}$ is estimated from knowledge of the model parameters and their correlation (Rodgers, 2000). Note that in this study the $\boldsymbol{S}_{p}$ matrix is diagonal and therefore defined as independent uncertainties rather than dependant uncertainties like $\boldsymbol{S}_{\text {obs }}$ (Rodgers, 2000). Incorporating these covariance matrices reduces the weight of observations and parameters with high uncertainty (Hakami et al., 2005).

The value of the regularisation parameter depends on the acceptable level of model prediction and assimilation errors. Figure 3.1.7.1 from Hakami et.al. (2005) shows this relationship, where an increase in the regularisation parameter decreases the model prediction error while simultaneously increasing the assimilation error. In their study, the regularisation parameter is chosen with respect to the minimum total error. The regularisation parameter chosen for assimilation varies between models and instrument retrievals. If assimilating multiple sources of instrument retrievals, each instrument may have a different regularisation 
parameter to weight their observations within the model. An example of this is seen in the MSA by X. Zhang et al. (2019), where GEOS-Chem experimental tests are run with differing regularisation parameters between retrieving satellites to find the optimal assimilation.

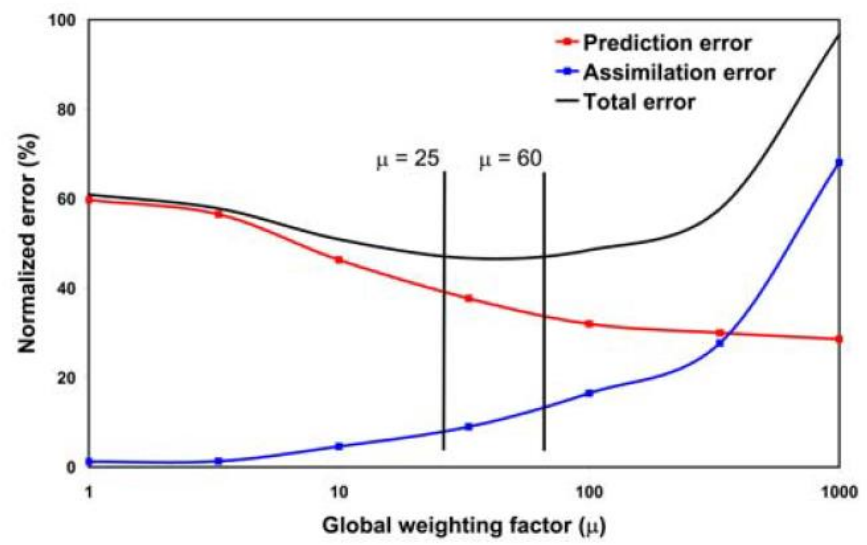

(Hakami et al., 2005)

Figure 3.1.7.1: Effect of the regularisation parameter $\gamma_{r}$ (shown here as global weighting factor $\mu$ ) on model prediction and assimilation error as demonstrated in Hakami et. al. (2005). At $\mu=1$, the model prediction error is equivalent to the initial model model error, and the assimilation error is equivalent to the observation error before assimilation.

\subsection{Part 1: CO intercomparison}

In Part 1 of this study, CO profile and total column observations from MOPITT, CrIS, and TROPOMI are evaluated against ground-based NDACC observations using the GEOS-Chem model as a common intercomparison platform. The vertical profiles of each observing platform are interpolated to common pressure levels so that cross-kernel calculation can be applied for proper intercomparison. This method removes bias from each respective instrument and is further explained in Section 3.2.1 below. The total columns are cross compared utilising a similar process. Differences between observed profiles are represented as root mean square error (RMSE), and differences between total columns are represented as percent differences. 


\subsubsection{CO retrieval and calculations in preparation for intercomparison}

Profile and total column CO observations are gathered from NDACC stations, MOPITT, TROPOMI, CrIS, Joint CrIS-TROPOMI, and GEOS-Chem model datasets. These profile datasets contain retrieval and modeled atmospheric information and CO concentrations from the Earth's surface to the defined top of atmosphere (TOA). It also includes information used to calculate a smoothed true concentration profile over the varying altitudes or pressures.

Eqn. (20) $\widehat{\boldsymbol{x}}=\boldsymbol{x}_{a}+\boldsymbol{A}\left(\boldsymbol{x}_{r}-\boldsymbol{x}_{a}\right)+\boldsymbol{\epsilon}_{x}$

where $\quad \hat{\boldsymbol{x}}=$ resulting smoothed profile

$$
\begin{aligned}
& \boldsymbol{x}_{a}=\text { a priori profile of observing platform } \\
& \boldsymbol{x}_{r}=\text { retrieved profile of observing platform } \\
& \boldsymbol{A}=\text { averaging kernel matrix of observing platform } \\
& \boldsymbol{\epsilon}_{x}=\text { error of } \hat{\boldsymbol{x}}
\end{aligned}
$$

Equation 20 (Rodgers \& Connor, 2003) is used for observing instruments to find a balance between the retrieved values and a prior state estimate, to create a profile closest to the true state. Here, the retrieved profile state vector $\boldsymbol{x}_{r}$ (size $n$ ) is the measured CO from the respective observing platform. The a priori $\boldsymbol{x}_{a}$ is a vector (size $n$ ) of the estimated state, usually based on historical or modeled values (Rodgers, 2000; Rodgers \& Connor, 2003). The error $\boldsymbol{\epsilon}_{x}$ of the resulting smoothed profile $\widehat{\boldsymbol{x}}$ is due to random retrieval error, instrument error, and forward calculation error (Rodgers, 2000; Rodgers \& Connor, 2003). The averaging kernel matrix $\boldsymbol{A}$ (size $n \times n$ ) typically contain unitless values between 0.0 and 1.0 , but can be negative 
where noise exceeds signal (Smith et al., 2020a). The averaging kernel is defined in Equation 21 (Deeter et al., 2015; Rodgers, 2000) and is a smoothing function that balances the measured and a priori values throughout the profile points (Rodgers, 2000). The gain matrix $(\boldsymbol{G})$ is a prescribed by the Jacobian matrix $(\boldsymbol{K})$, radiance error covariance matrix $\left(\boldsymbol{S}_{\epsilon}\right)$, and a priori covariance matrix $\left(\boldsymbol{S}_{a}\right)$ (Deeter et al., 2015; Rodgers, 2000). The Jacobian matrix "describes the sensitivity of the measurements to the actual vertical profile", $\boldsymbol{S}_{\epsilon}$, and $\boldsymbol{S}_{a}$ (Deeter et al., 2015). Wherein $\boldsymbol{S}_{\epsilon}$ and $\boldsymbol{S}_{a}$ represent measurement uncertainties and background variability respectively, along with their correlations (Deeter et al., 2015; Rodgers, 2000).

$$
\text { Eqn. (21) } \quad \boldsymbol{A}=\boldsymbol{G} \boldsymbol{K}=\left(\boldsymbol{K}^{T} \boldsymbol{S}_{\epsilon}^{-1} \boldsymbol{K}+\boldsymbol{S}_{a}^{-1}\right)^{-1} \boldsymbol{K}^{T} \boldsymbol{S}_{\epsilon}^{-1} \boldsymbol{K}
$$

$$
\begin{aligned}
& \text { where } \quad \boldsymbol{G}=\text { gain matrix } \\
& \boldsymbol{K}=\text { weighting function matrix (Jacobian matrix) } \\
& \boldsymbol{S}_{\epsilon}=\text { radience error covariance matrix } \\
& \boldsymbol{S}_{a}=\text { a priori covarience matrix }
\end{aligned}
$$

The closer the averaging kernel values are to zero, the less confidence there is in the instrument's observation and the more heavily weighted the a priori becomes (Smith \& Barnet, 2020). The closer the value is to one, the more confidence there is in the instrument observation (Smith \& Barnet, 2020). In other terms, the averaging kernel describes the dependence of the retrieved state vector on the true smoothed vector (Francis et al., 2017). Figure 3.2.1.1 is an example of an averaging kernel for the Toronto NDACC instrument. The trace of the averaging kernel provides the "degrees of freedom for signal" (DOFS or DFS), which 
is a "metric for information content" interpreted as "the number of independent pieces of information contained in the retrieval resulting from the actual observation" (Deeter et al., 2015). This is not be confused with the "degrees of freedom for noise", or the "degrees of freedom" which is the DOFS and "degrees of freedom for noise" together (Rodgers, 2000). The DOFS is "a measure of resolution", where the higher the number the greater the information content (Deeter et al., 2015; Rodgers, 2000).

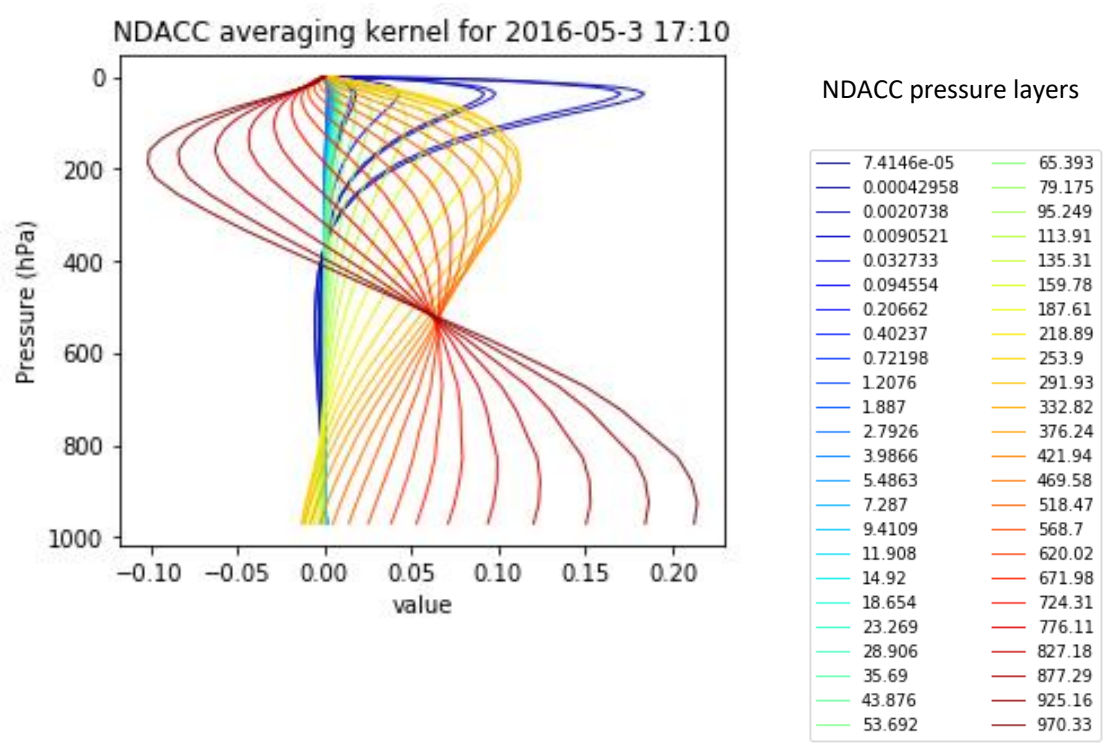

Figure 3.2.1.1: A unique Toronto NDACC station CO averaging kernel for May $3^{\text {rd }} 2016$, where each matrix row is plotted as a NDACC pressure layer represented in the legend.

As described in Section 3.1, MOPITT, TROPOMI, CrIS, and NDACC all use different sources for their a priori values and consequently also have unique averaging kernels to weight them. Therefore, each observing platform's smoothed profile values are dependant on their individual unique assumed state, cannot be compared directly, and must be reconstructed first (Rodgers \& Connor, 2003). To cross-compare the differing smoothed profiles, each instrument must be evaluated using the a priori and averaging kernel of the other instrument as if looking 
though the lens of the other instrument. Equation 22 (Rodgers \& Connor, 2003) shows how the a priori and averaging kernel of one instrument is applied to the smoothed profile of another.

This reduces the smoothing error when comparing profiles (Rodgers \& Connor, 2003). Figure

3.2.1.2 contains example plots of CO profiles before and after applying Equation 22, where instrument 1 is the NDACC instrument.

Eqn. (22) $\widehat{\boldsymbol{x}}_{12}=\boldsymbol{x}_{a 1}+\boldsymbol{A}_{1}\left(\widehat{\boldsymbol{x}}_{2}-\boldsymbol{x}_{a 1}\right)$

where $\quad \widehat{\boldsymbol{x}}_{12}=$ resulting cross - kernel profile, instrument 2 as instument 1

$\boldsymbol{x}_{a 1}=$ a priori profile of instrument 1

$\widehat{\boldsymbol{x}}_{2}=$ retrieved smoothed profile of instrument 2

$\boldsymbol{A}_{1}=$ averaging kernel matrix of instument 1
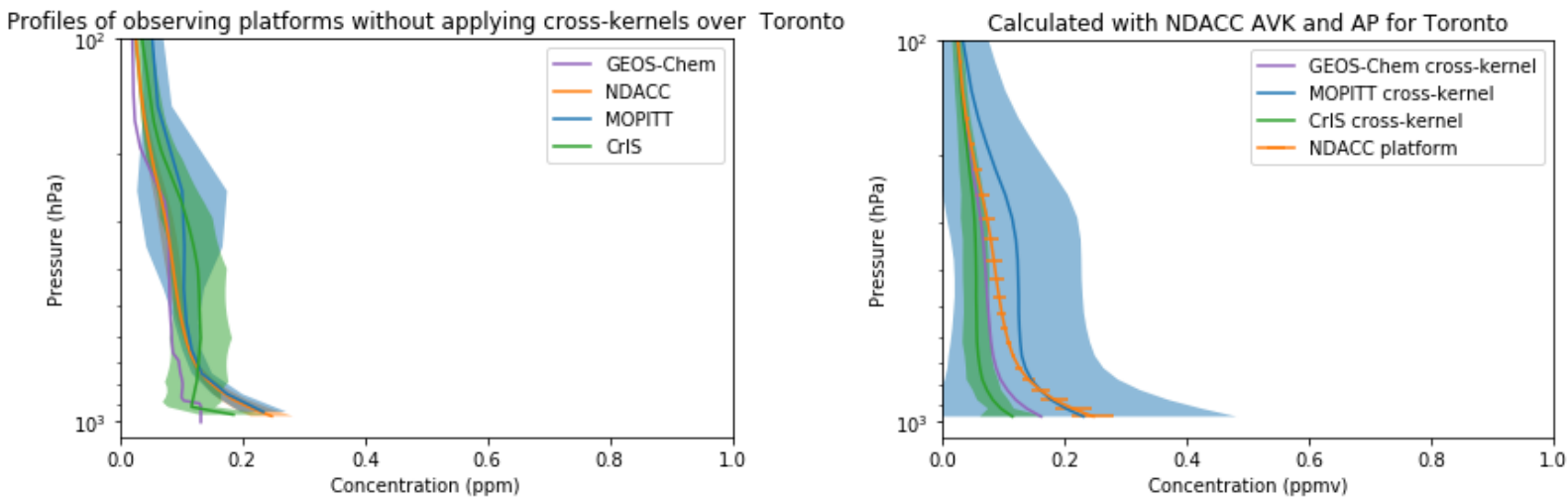

Figure 3.2.1.2: (Left) Smoothed CO profile retrievals of each instrument over Toronto for May $3^{\text {rd }}$ with no cross-kernels applied. (Right) Cross-kernel smoothed profiles for NDACC over Toronto for May $3^{\text {rd }}$. The NDACC smoothed profile and error are shown in orange. The NDACC a priori and averaging kernel are applied to the smoothed retrievals of the other observing platforms. 
For Part 1 of this study, CO profiles are compared between instruments by applying MOPITT, CrIS and NDACC a priori and averaging kernels to profiles of the other observing platforms. The TROPOMI profile comparisons are not included as only total column information is accessible. The NDACC and MOPITT a priori and averaging kernels are readily available for use within the Level 2 datasets. The CrIS CLIMCAPS Level 2 product does not contain a priori or full 100-level averaging kernels within its datasets, but the user can calculate them separately as shown below.

The CLIMCAPS a priori product is calculated from two static 12-month climatology profiles (one NH and one SH) based on MOPITT (Smith et al., 2020b). The a priori of each data point is calculated by weighting latitude and date between the two files (Smith et al., 2020b). This method and the climatology files are further described in the CLIMCAPS CO application users guide provided by Nadia Smith (Smith et al., 2020b).

Smith et al. (2002b) clarifies that the averaging kernel provided in the CLIMCAPS dataset is on a reduced set of overlapping pressure layers called trapezoid state functions for reduced product size and faster calling for optimal estimation forward models. CLIMCAPS' CO retrieval has 9 trapezoid state functions for 9 pressure hinge-points, defining the pressure levels at which the averaging kernels are calculated (Smith et al., 2020a). Equations 23 and 24 (Smith et al., 2020a) define how this information is used to calculate the unique averaging kernels for each retrieval scene, up to 100 levels. This method is further described in the CLIMCAPS Uncertainty Application Guide (Smith et al., 2020a).

Eqn. (23) $\quad \boldsymbol{A}_{n \times n}=\boldsymbol{F}_{L \times j}^{T} \boldsymbol{A}_{L \times L} \boldsymbol{F}_{L \times j}^{+}$ 
Eqn. (24) $\quad \boldsymbol{F}_{L \times j}^{+}=\left[\boldsymbol{F}_{L \times j} \boldsymbol{F}_{L \times j}^{T}\right]^{-1} \boldsymbol{F}_{L \times j}$

where $\quad n=$ the number of levels in the CLIMCAPS retrieval scene

$L=$ the number of trapezoid layers

$\boldsymbol{A}_{n \times n}=$ resulting averaging kernel, shape $n \times n$

$\boldsymbol{A}_{L \times L}=$ averaging kernel matix on trapezoid layers, shape $L \times L$

$\boldsymbol{F}_{L \times j}=$ transformation matrix reconstructed from

hinge points, shape $L \times j$

$\boldsymbol{F}_{L \times j}^{+}=$pseudo inverse of $\boldsymbol{F}_{L \times j}$

Like the CO profiles, the CO total columns should not be directly compared either (Rodgers \& Connor, 2003). Total columns also have their own unique a priori and averaging kernels, where the a priori are scalars and the averaging kernels are vectors (Rodgers \& Connor, 2003). Equation 25 shows how the a priori and averaging kernel of one instrument is applied to the total column of another (Rodgers \& Connor, 2003).

Eqn. (25) $\hat{c}_{12}=c_{a p 1}+\boldsymbol{k}_{1}^{T}\left(\hat{\boldsymbol{x}}_{2}-\boldsymbol{x}_{a p 1}\right)$

where $\quad \hat{c}_{12}=$ resulting cross - kernel total column, insrument 2 as instrument 1

$c_{a p 1}=$ a priori total column of instrument 1

$\boldsymbol{x}_{a p 1}=$ a priori profile of instument 1 


$$
\begin{aligned}
& \widehat{\boldsymbol{x}}_{2}=\text { retrieved profile of instument } 2 \\
& \boldsymbol{k}_{1}=\text { total column averaging kernel vector of instrument } 1
\end{aligned}
$$

NDACC and MOPITT datasets contain ready to use total column a priori and averaging kernels. However, the TROPOMI dataset does not contain profile a priori information required by Equation 25 for the cross-kernel calculation. The CrIS dataset contains information for the total column a priori, but not the averaging kernel. Therefore, only NDACC and MOPITT crosskernels were possible with each observing platform apart from TROPOMI. Table 3.2.1.1 below summarises the possible cross-kernel evaluations between each observing platform, depending on the availability of CO profile and total column a priori and averaging kernels for each retrieval.

Table 3.2.1.1: Summary of prepared cross-kernels for intercomparison. " $P$ " and " $T$ " indicate where profile and total column

\begin{tabular}{|c|c|c|c|c|c|}
\hline & \multicolumn{4}{|c|}{ Instrument 1} \\
\hline & & NDACC & MOPITT & CrIS & TROPOMI \\
\hline \multirow{5}{*}{ 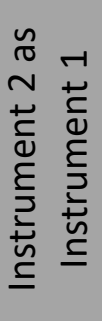 } & NDACC & -- & $\mathrm{P}$ and $\mathrm{T}$ & P only & Neither \\
\hline & MOPITT & $\mathrm{P}$ and $\mathrm{T}$ & -- & P only & Neither \\
\hline & CrIS & $\mathrm{P}$ and $\mathrm{T}$ & $\mathrm{P}$ and $\mathrm{T}$ & -- & Neither \\
\hline & TROPOMI & Neither & Neither & Neither & -- \\
\hline & GEOS-Chem & $\mathrm{P}$ and $\mathrm{T}$ & $\mathrm{P}$ and $\mathrm{T}$ & P only & Neither \\
\hline
\end{tabular}
cross-kernels could be calculated, respectively.

\subsubsection{CO intercomparison}

The NDACC station data at different locations are used to verify and compare the various satellite and GEOS-Chem model CO accuracy within the troposphere. However, each observing platform has a different FOV or area of measurement. NDACC station data are taken 
at different times from a single point location. MOPITT, TROPOMI and CrIS data used are collections of FOV point data taken at different times and locations around the globe. GEOSChem data is a collection of data over large gridded areas covering the globe.

For cross-comparison between all platform profiles and total columns, coinciding times and locations were used for May through August in 2016 primarily. At the start of this research, only full datasets up to 2016 were available for all NDACC stations. Therefore, 2016 NDACC, MOPITT, and GEOS-Chem runs were selected for this study's analysis. However, since the earliest usable TROPOMI data file is from July 2018 (Copernicus Sentinel-5P (processed by ESA), 2018), 2019 TROPOMI data was used instead of 2016 or 2018 data. CrIS 2019 data was also selected as it is within the same timeframe as TROPOMI, and it was discovered that the instrument failed to transmit mid-IR information during the 2016 timeframe (N. Smith, personal communication, September 15,2020$)$. In summary, the profile and total column comparisons from NDACC, MOPITT and GEOS-Chem 2016 are compared with CrIS and TROPOMI 2019 data. May through August was selected as $\mathrm{OH}$ concentrations are higher during these months in the Northern Hemisphere, where the majority of the NDACC stations located.

For data comparison within the same time frame, overlapping times within \pm 4 hours of a daily NDACC station measurement were used. The daily selected NDACC time for comparison was the closest station time to the average daily TROPOMI overpass time. This was selected due to JPSS-1 (CrIS) and Sentinel-5P's (TROPOMI) global daily coverage, close proximity in orbit, and consistent overpass time (JPSS, n.d.). The \pm 4 hour window is selected to allow more data inclusion from MOPITT as it has an inconsistent satellite overpass time over each NDACC station, due to its $\sim 3$ day global coverage (Drummond et al., 2010). Therefore, the CrIS, 
TROPOMI, and GEOS-Chem time difference with the selected NDACC station data is minimal compared to MOPITT.

For data comparison within the same geographical area, MOPITT, TROPOMI and CrIS data were averaged over a $200 \mathrm{~km}$ radius from each respective NDACC station. The resulting variability from averaging is added to the overall total profile and total column errors. For crosskernel profiles and total columns, averaging was applied after using Equation 22 or 25 . A smaller radius was not chosen as it severely minimized or eliminated the available CrIS and MOPITT datapoints due to low quality or missing data. For GEOS-Chem, data from the single grid cell containing the NDACC station location was selected for intercomparison. Note that the GEOS-Chem grid is a rectilinear latitude-longitude grid with a spatial resolution of $2^{\circ} \times 2.5^{\circ}$ and is not determined by true distances. Therefore, the single grid cell selected may not necessarily well represent the $200 \mathrm{~km}$ radius of satellite values surrounding an NDACC station. For visual aid, Figure 3.2.2.1 below is a global map of all NDACC stations locations in this study with their $200 \mathrm{~km}$ radius. Figure 3.2.2.2 is a closer view over Europe with the GEOS-Chem rectilinear grid, demonstrating the difference in number of grid cells within a station's $200 \mathrm{~km}$ radius depending on global position. 


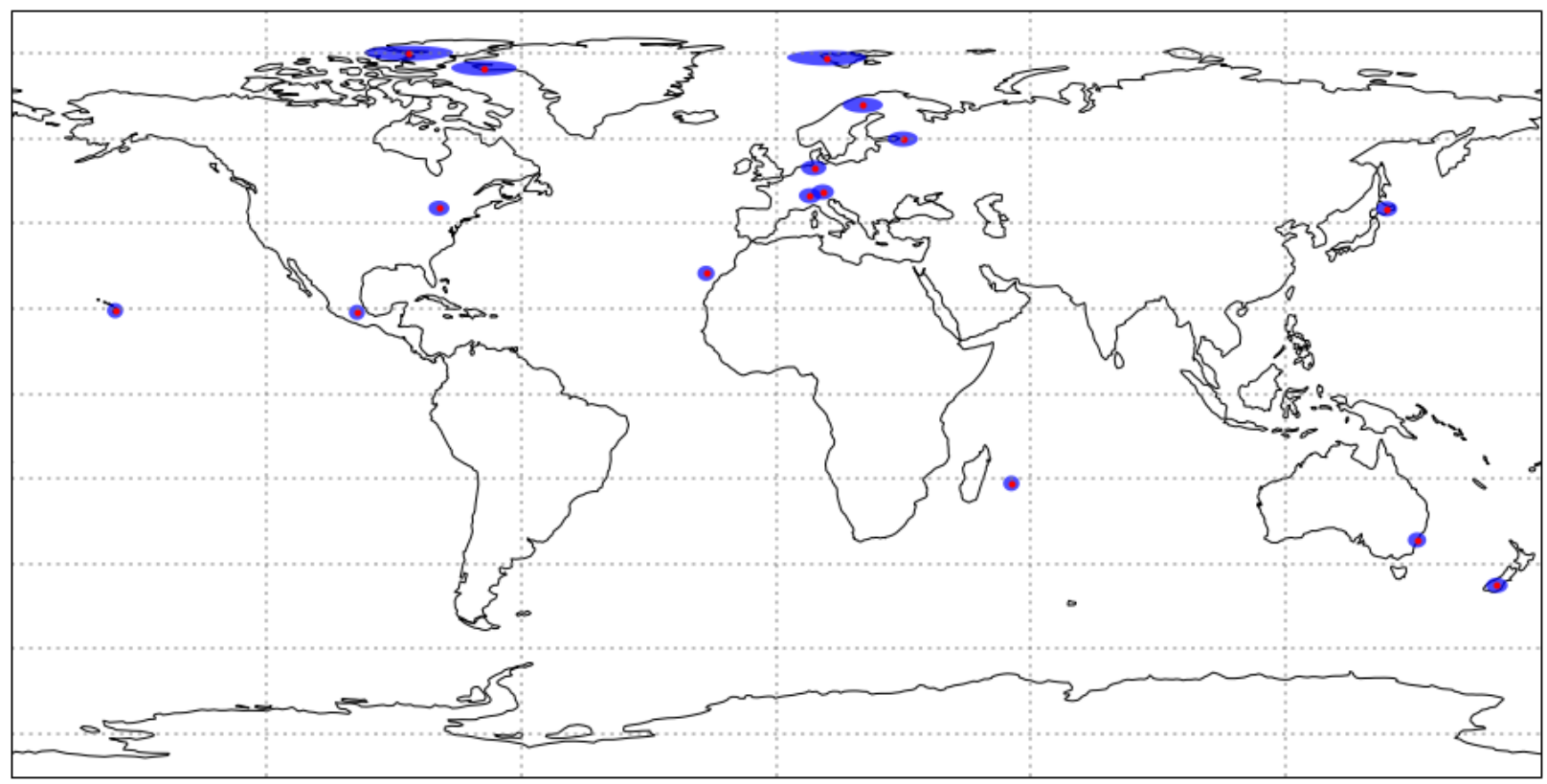

Figure 3.2.2.1: Global map of all NDACC station locations utilised in this study (red dots), and their $200 \mathrm{~km}$ radius (blue).

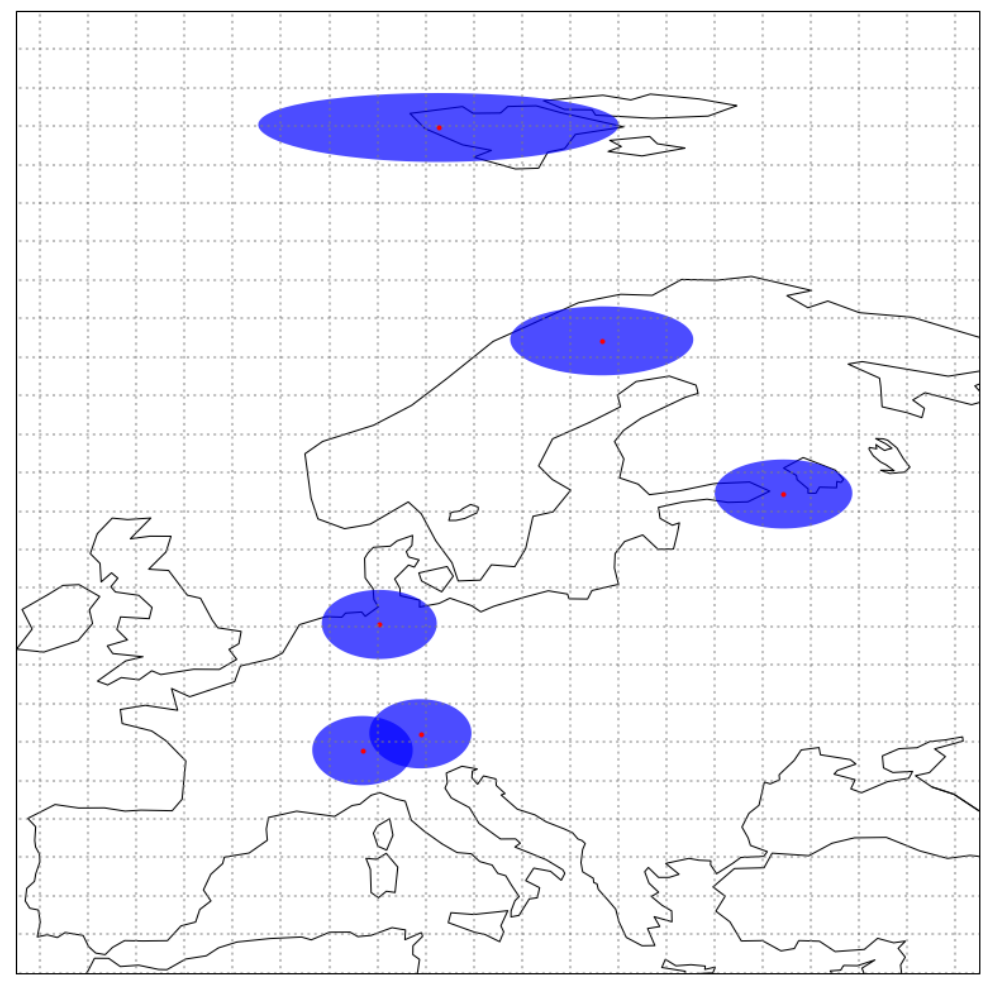

Figure 3.2.2.2: Map of select NDACC stations utilised in the study over Europe (red dots), their $200 \mathrm{~km}$ radius (blue), and the GEOS-Chem rectilinear $2^{\circ} \times 2.5^{\circ}$ grid. Note the difference in number of grid cells within the $200 \mathrm{~km}$ radius of each station. 
The overlapping CO profiles from each observing platform are recorded at different pressure levels and layers in the atmosphere. For cross-kernel comparison, each instrument's smoothed profile is interpolated to the levels or layers of the other's so that Equations 22 and 25 may be applied. These interpolated profiles are created using the Python package scipy.interpolate. UniverariateSpline fitted through each point (smoothing factor $s=0.0$ ), with a few exceptions. These smoothing factor exceptions include the CrIS interpolation to MOPITT levels for Zugspitze and Jungfraujoch, and the CrIS interpolation to NDACC layers for Thule where a smoothing factor of $s=0.5$ and $s=0.7$ are used respectively. It was discovered that although scipy.interpolate.UniverariateSpline was able to accurately interpolate between profile level/layer points, it had a tendency to overfit extrapolated values at the ends of the splines. To minimise these atypical overfitting occurrences, the noted smoothing factor adjustments were implemented for select stations. Without these adjustments, the surface concentration extrapolation tended to be larger than was physically reasonable.

It is also noted that not all data products provide CO profile and total column information in the same units, or provide the same information about the atmospheric state. Units are required to be converted for cross-comparison. Table 3.2.2.1 summarises the units and atmospheric state information provided by each observing platform. 
Table 3.2.2.1: Summary of observing platforms' units and atmospheric state information provided in their data products.

\begin{tabular}{|c|c|c|c|c|c|}
\hline Parameter & NDACC & MOPITT & $\begin{array}{c}\text { CrIS } \\
\text { (CLIMCAPS) }\end{array}$ & TROPOMI & GEOS-Chem \\
\hline CO profile & ppm & $\mathrm{ppb}$ & $\mathrm{molec} / \mathrm{m}^{2}$ & -- & $\mathrm{ppb}$ \\
\hline $\begin{array}{l}\text { CO total } \\
\text { column }\end{array}$ & $\mathrm{molec} / \mathrm{cm}^{2}$ & $\mathrm{molec} / \mathrm{cm}^{2}$ & $\mathrm{molec} / \mathrm{m}^{2}$ & $\mathrm{molec} / \mathrm{m}^{2}$ & -- \\
\hline $\begin{array}{l}\text { Pressure } \\
\text { profile }\end{array}$ & $\mathrm{hPa}$ & $\mathrm{hPa}$ & $\mathrm{Pa}$ & -- & $\mathrm{hPa}$ \\
\hline $\begin{array}{l}\text { Altitudes } \\
\text { profile }\end{array}$ & $\mathrm{km}$ & $\begin{array}{l}\text { Not provided. } \\
\text { Derived from } \\
\text { CAM-Chem } \\
\text { interpolation }\end{array}$ & $\mathrm{m}$ & -- & $\mathrm{km}$ \\
\hline $\begin{array}{l}\text { Temperature } \\
\text { profile }\end{array}$ & K & $\begin{array}{l}\text { Not provided. } \\
\text { Derived from } \\
\text { CAM-Chem } \\
\text { interpolation }\end{array}$ & K & -- & N/A \\
\hline $\begin{array}{l}\text { Air density } \\
\text { profile }\end{array}$ & $\begin{array}{l}\text { Calculated } \\
\text { from NDACC } \\
\text { pressure, } \\
\text { altitude, and } \\
\text { temperatures }\end{array}$ & $\begin{array}{l}\text { Not provided. } \\
\text { Calculated } \\
\text { from } \\
\text { pressure, } \\
\text { CAM-Chem } \\
\text { altitude and } \\
\text { temperature } \\
\text { interpolations }\end{array}$ & $\begin{array}{l}\text { Calculated } \\
\text { from } \\
\text { CLIMCAPS } \\
\text { pressure, } \\
\text { altitude, and } \\
\text { temperatures }\end{array}$ & -- & $\mathrm{molec} / \mathrm{cm}^{3}$ \\
\hline
\end{tabular}

Where air density was not provided, it was calculated using pressure, altitude, and temperature profiles. First the surface air density is calculated using provided surface values using Equation 26 (Bohren \& Albrecht, 1998). Next, the air density of each subsequent layer above is calculated using the calculated air density and other values from the layer below using Equation 27 (Bohren \& Albrecht, 1998). The air density and altitudes were then used to convert between VMRs and concentrations in molecules $/ \mathrm{m}^{2}$, so the cross-kernel Equation 22 could be applied. 
Eqn. (26)

$$
\rho_{s}=\left[\frac{P_{s}}{\left(\frac{R}{M_{d a}}\right) T_{s}}\right]
$$

where

$$
\begin{aligned}
& \rho_{s}=\text { the dry air density at the surface }\left(\mathrm{kg} / \mathrm{m}^{2}\right) \\
& P_{S}=\text { the air pressure at the surface }(\mathrm{Pa}) \\
& R=\text { the universal gas constant }=8.314462618(\mathrm{~N} \cdot \mathrm{m}) /(\mathrm{mol} \cdot \mathrm{K}) \\
& M_{d a}=\text { the molar mass of dry air }=0.0289644 \mathrm{~kg} / \mathrm{mol} \\
& T_{S}=\text { the temperature at the surface }(\mathrm{K})
\end{aligned}
$$

Eqn. (27)

$$
\rho_{2}=\rho_{1}\left[\frac{T_{1}}{T_{1}+L_{1}\left(h_{2}-h_{1}\right)}\right]^{\left(1+\frac{g M_{d a} a}{R L_{1}}\right)}
$$

where

$$
L_{1}=-\frac{T_{2}-T_{1}}{h_{2}-h_{1}}
$$

1 denotes the layer/level below, and 2 denotes the layer

$$
\text { /level being calculated }
$$

$$
\rho=\text { the layer/level dry air density }\left(\mathrm{kg} / \mathrm{m}^{2}\right)
$$

$L=$ the layer/level temperature lapse rate

$h=$ the layer /level altitude $(m)$

$T=$ the layer/level temperature $(K)$

$g=$ gravitational acceleration $=9.80665 \mathrm{~m} / \mathrm{s}^{2}$ 
Notably, the calculation of air density to convert between units can introduce error. As shown in Table 3.2.2.1, the MOPITT data product does not provide information required to calculate the air density profile. Here, altitude and temperature profiles are interpolated from the CAM-Chem data product, adding additional possible errors. These errors are encompassed in the calculated variability of the cross-kernel products for each day. This variability is added to the interpolated error for each profile and total column for a total error. Profile total error is displayed as error bars and the shaded area about each graphed CO profile (Figure 3.2.1.2). Another source of potential error when cross comparing is variability in surface elevation around each NDACC station. For example, the Mauna Loa station is located at an elevation of $3397 \mathrm{~km}$ (NDACC, 2021), and a $200 \mathrm{~km}$ radius would include data ranging down to sea level (Center for International Earth Science Information \& Network(CIESIN)/Columbia University, 2021). Therefore, the cross-kernel with NDACC would be limited to above the NDACC station while the MOPITT, CrIS and TROPOMI profiles and total columns may include averaged CO concentrations down to sea level. It should also be noted that the MOPITT data product does not contain a surface altitude variable. For the purpose of these calculations, the surface altitude value was drawn from CAM-Chem. Where the CAM-Chem surface elevation was greater than that of the NDACC station, the NDACC station elevation is assumed.

The final cross-kernel total columns are compared in terms of percent difference and absolute difference. The percent difference calculated as each observing platform's total column subtracted from base instrument (NDACC cross-kernel or MOPITT cross-kernel) total column, divided by the mean between the two values. Differences between the final crosskernel profiles are compared with root mean square error (RMSE) in ppm to compare how 
similar the profile concentrations and shape are to the base profile. RMSE was chosen over a correlation coefficient as the correlation coefficient is relative to the instruments being compared, whereas RMSE provides an absolute measurement that can be compared across all instruments. RMSE was also used over percent difference so that the profile differences can be represented as a single value rather than multiple values for over the different levels, particularly for the instruments with greater numbers of vertical levels. To assess differences at different vertical levels within the atmosphere, RMSE is calculated across four defined pressure ranges: TOA to surface, $100 \mathrm{hPa}$ to surface, $100 \mathrm{hPa}$ to the approximate boundary layer pressure, and the approximate boundary layer pressure to the surface. The RMSE was calculated over these four ranges as the various observing platform profiles have varying observing capabilities. MOPITT retrieval profiles only range from $100 \mathrm{hPa}$ to the surface, hence the $100 \mathrm{hPa}$ division. It is noted in the CLIMCAPS application guide that "CLIMCAPS has sensitivity to CO within the vertical layer roughly from $200 \mathrm{hPa}$ to $700 \mathrm{hPa}$ ", therefore a "weak sensitivity to CO in the boundary layer" (Smith et al., 2020b). Thus, another pressure layer division around the top of the boundary layer at approximately $800 \mathrm{hPa}$. The $800 \mathrm{hPa}$ is chosen over $900 \mathrm{hPa}$ (PBL top at $3 \mathrm{~km}$ (Jacob, 2000)), as this would allow for 2 profile RMSE points for MOPITT cross-comparison rather than just a single point.

The profile RMSE and total column differences are then averaged monthly for comparison as NDACC data completeness varied greatly by station. An overall mean is also calculated using these four monthly average values. Missing data spans can range from days to months. The trace of the averaging kernels, known as the degrees of freedom for signal (DOFS 
or DFS), are also compared on a monthly average between instruments. The DOFS indicates the number of layers that the instrument is sensitive to in the vertical profile (Smith et al., 2020b).

\subsection{Part 2: Sensitivities}

The global atmospheric GEOS-Chem Adjoint (version: v35m) model is used to establish an assumed global baseline state in terms of the concentrations of atmospheric trace gases. Part 2 investigates the modeled differences between the GEOS-Chem baseline and the integrated MOPITT GEOS-Chem model outputs for CO abundance and distribution. It also compares the $\mathrm{CO}$ emission sensitivities, and the $[\mathrm{CH} 4+\mathrm{OH}]$ reaction sensitivity of the NDACC network compared to the global MOPITT observations. These sensitivities provide insight into instrument measurements and characteristics, and ultimately their capabilities to constrain $\mathrm{OH}$ concentrations based on observed CO concentrations.

First, the GEOS-Chem adjoint model was run for each month from May through August 2016. The GEOS-Chem adjoint model was run next, integrating MOPITT 2016 observations. The adjoint model adjusts the CO parameters to more closely represent MOPITT observations. The more adjoint iterations, the more the GEOS-Chem model will match MOPITT observations. MOPITT v7 product assimilation is already included within the GEOS-Chem model settings. The MOPITT profiles are averaged and represented in the GEOS-Chem grid using Equation 19 in Section 3.1.7, with a default regularisation parameter of 1.0. Multiple iterations were attempted, although the outputs revealed a compounding error with each iteration. For this study, only the first iteration MOPITT adjoint outputs are analysed. This limits the sensitivity analysis to the initial model response rather than a completely developed sensitivity after 
MOPITT CO observations are fully assimilated with a minimised cost function. The difference between the GEOS-Chem baseline and MOPITT integrated CO are then compared in vertical and horizontal geographical plots.

Second, the sensitivities of the MOPITT observations and the NDACC network observations to $\mathrm{CO}$ anthropogenic, biomass burning, and biofuel emissions are compared. This examines the sensitivity of each model grid cell where this is an observation to surrounding CO emissions, impacting $\mathrm{CO}$ and ultimately $\mathrm{OH}$ outputs. Positive sensitivity represents a model response where the cost function is increasing due to increasing changes in CO emissions. Negative sensitivity represents a model response where cost function is decreasing due to increasing changes in $\mathrm{CO}$ emissions. The cost function for the MOPITT observations and that for the NDACC network differ, complicating the interpretation of the sensitivities.

For the base GEOS-Chem model with NDACC station grid cells selected, the cost function is equivalent to the column-averaged CO concentration above each NDACC station. Therefore, positive sensitivity represents an increase in model $\mathrm{CO}$ concentration due to increasing changes in emissions. No negative sensitivities are present as there is no model assimilation response affecting the cost function. However, the MOPITT CO emission sensitivities represent the model assimilation response, with regards to the cost function explained in Section 3.1.7. Looking at the relationships in Equation 19 for NDACC, a positive sensitivity indicates that increasing CO emissions will increase the cost function. This means that the difference between observed and simulated concentrations are increasing due to increasing $\mathrm{CO}$ emissions. Therefore, the model response will be to reduce model $\mathrm{CO}$ emissions. A negative sensitivity indicates the opposite, where the model response will be to increase the model CO emissions. 
Third, the $\left[\mathrm{CH}_{4}+\mathrm{OH}\right]$ reaction sensitivity outputs of both adjoint models are compared. This reveals the chemical sensitivity of each grid cell, impacting $\mathrm{CO}$ and $\mathrm{OH}$ outputs. Note the GEOS-Chem simulation does not simulate $\mathrm{OH}$ in the stratosphere, therefore these reactions are limited to the troposphere in the model. Positive sensitivity represents a model response where the cost function is increasing due to increasing changes in $\left[\mathrm{CH}_{4}+\mathrm{OH}\right]$ reaction rate, which we can take as a proxy for the $\mathrm{OH}$ concentration assuming that methane concentrations are relatively well-known. Negative sensitivity represents a model response where the cost function is decreasing due to increasing changes in $\left[\mathrm{CH}_{4}+\mathrm{OH}\right]$ reaction rate.

For the base GEOS-Chem model with NDACC station grid cells selected, positive sensitivities indicate an increase in $\mathrm{CO}$ concentrations due to increasing changes in $[\mathrm{CH} 4+\mathrm{OH}]$ reaction rate. For the MOPITT assimilation, a positive sensitivity again indicates an increase in cost function due to increasing changes in the reaction rate. This means that the difference between observed and simulated concentrations are increasing due to increasing reaction rates. Therefore, the model response will be to reduce the modeled reaction rate. Again, a negative sensitivity indicates the opposite, where the model response will be to increase the model reaction rate. Differences between the MOPITT and GEOS-Chem base modeled sensitivities provide spatial information on model adjustments made to generate CO outputs.

Modeled sensitivities and CO outputs in this study are limited to the GEOS-Chem baseline, NDACC and MOPITT integrated platforms due to time constraints and the need for additional model development. Additional observation operator code development is required for CrIS and TROPOMI assimilation. It is recommended in further studies that multiple adjoint iterations be conducted for each observing platform including the CrIS CLIMCAPS, TROPOMI, 
and eventually the Joint CrIS-TROPOMI CO data products to provide further insight into instrument measurements and characteristics in determining global $\mathrm{OH}$.

\subsection{Part 3: $\mathrm{OH}$ evaluation}

Like in Part 2, the global atmospheric GEOS-Chem Adjoint (version: v35m) model is used to establish an assumed global baseline state. Part 3 investigates the modeled differences between the GEOS-Chem baseline and the integrated MOPITT GEOS-Chem model outputs for $\mathrm{OH}$ abundance and distribution. Due to compounding error throughout multiple adjoint iterations, only the first iteration outputs are compared. Again, this first iteration can only provide initial insight to the model response and is not representative of a completely developed output after MOPITT CO assimilation with a minimised cost function. The difference between the GEOS-Chem baseline and MOPITT integrated $\mathrm{OH}$ are compared in vertical and horizontal geographical plots.

The global, Northern Hemisphere, and Southern Hemisphere mean $\mathrm{OH}$ are calculated for comparison with past literature. These values are also used to calculate the $\mathrm{NH} / \mathrm{SH} \mathrm{OH}$ ratio for comparison. As noted, the GEOS-Chem simulation does not simulate $\mathrm{OH}$ in the stratosphere (above the $38^{\text {th }}$ vertical model layer) and is therefore limited to the troposphere within the model. Past studies discussed in the literature review generally calculate the $[\mathrm{OH}]_{\mathrm{Gm}}$ below 200 $\mathrm{hPa}, 100 \mathrm{hPa}$, the tropopause, or up to the TOA (see Table 2.3.1.1). Note, an $\mathrm{OH}$ comparison below $100 \mathrm{hPa}$ would include some GEOS-Chem stratospheric layers, weighting the average lower since $\mathrm{OH}$ is not calculated by the model in the stratosphere. These studies also generally provide $[\mathrm{OH}]_{\mathrm{GM}}$ in airmass, mass, or volume weighted values. For comparison with literature, 
this study reports monthly average airmass, mass (CO), and volume weighting $[\mathrm{OH}]_{\mathrm{GM}}$ from May through August 2016 below $100 \mathrm{hPa}$.

Modeled $\mathrm{OH}$ outputs in this study are limited to the GEOS-Chem baseline and MOPITT integrated platforms due to time constraints. It is recommended for further studies that multiple adjoint iterations be conducted for each observing platform including the CrIS CLIMCAPS, TROPOMI, and eventually the Joint CrIS-TROPOMI CO data products to provide further insight into instrument differences in determining global $\mathrm{OH}$. Multiple iterations may also allow insight into correlations and relationships between $\mathrm{CO}, \mathrm{OH}$, and sensitivities within the calculated model space and how GEOS-Chem assimilation system responds to the different instrument inputs. 


\subsection{Observations and Discussion}

This section overviews observations and investigations into the three parts of this study. In Part 1 (Section 4.1), satellite CO total column and profiles are compared against the NDACC station network and the GEOS-Chem base model using total column percent difference, and profile RMSE. This CO intercomparison between instruments examines their precision and accuracy, while addressing the extent of bias and error. In Part 2 (Section 4.2), the GEOS-Chem adjoint is used to determine the sensitivities of NDACC locations and a single iteration assimilation of MOPITT to $\mathrm{CO}$ emissions and the $\left[\mathrm{CH}_{4}+\mathrm{OH}\right]$ reaction rate. This provides an initial look into the impact and extent these two CO datasets have on the GEOS-Chem model's output. In Part 3 (Section 4.3), the GEOS-Chem base model global OH is compared against the global $\mathrm{OH}$ from the single iteration MOPITT assimilation. This single iteration provides an initial look into model adjustments made on global $\mathrm{OH}$, as impacted by MOPITT $\mathrm{CO}$ assimilation.

Observations from Part 1 revealed that the MOPITT satellite still provides greatest CO total column and full profile accuracy when compared to NDACC. CrIS provides high resolution accuracy only against high elevation NDACC stations, as CrIS's sensitivity is best at elevations above $700 \mathrm{hPa}$ (Smith et al., 2020b). TROPOMI has a comparative total column against MOPITT, however without a proper cross-kernel calculation analysis the value of this observation is limited. Sensitivities from Part 2 reveal MOPITT's greater impact on the GEOS-Chem model than NDACC. Therefore, MOPITT currently offers the best CO accuracy for assimilation with GEOSChem to infer $\mathrm{OH}$ in Part 3. However, a future Joint CrIS-TROPOMI product has the potential to offer even greater spatial distribution and regional insight. 


\subsection{Part 1: CO intercomparison}

Observations of Part 1 include evaluating total column and profile cross-kernel comparisons between each observing platform and their respective DOFS values throughout the months May through August. Total columns are compared using percent difference (Section 4.1.1), profiles using RMSE (Section 4.1.2), and DOFS using their distribution (Section 4.1.3). Section 4.1.4 is a discussion on the observations including overall performance of the various instruments with respect to each other. Section 4.1.5 considers potential sources of error and bias, and the effect they may have on these observations. In the end, the intercomparison analysis found that the MOPITT CO profiles and total columns have the best overall fit to the NDACC network observations within the troposphere. The CrIS CO profile has high agreement with NDACC, and comparable values to MOPITT above the boundary layer. TROPOMI CO total columns were found to have high agreement with the MOPITT total columns, although this analysis is limited as proper cross-kernel evaluations were not possible.

\subsubsection{Total column comparison observations}

This section discusses the total column cross-kernel comparisons, where each observing platform "looks through the lens" of NDACC and MOPITT. As mentioned in Table 3.2.1.1, total column cross-kernel calculations were only possible for NDACC and MOPITT. Figure 4.1.1.1 below displays the four-month mean percent difference between the $\mathrm{CO}$ total columns of the different observing platforms. Note that the four-month mean is calculated from the four monthly average values May through August, where each month is equally weighted. This Figure includes two different percent difference comparisons against NDACC station values, one with no cross-kernel applied (left) and one with the NDACC cross-kernel calculation applied 
(middle). It also includes the percent difference comparisons against MOPITT cross-kernel total columns (right).

Figures 4.1.1.2 and 4.1.1.3 present the monthly mean percent difference values of each NDACC station for the NDACC and MOPITT total column percent differences, respectively. Note that for all total column figures, the TROPOMI percent difference presented in all graphs are not derived from cross-kernel calculations since required values were not available for the cross-kernel calculation (see Section 3.2.1). TROPOMI is included for comparison but has limited significance without the cross-kernel calculations applied.

In Figure 4.1.1.1, six of the NDACC station locations (Altzomoni, Izana, Jungfraujoch, Kiruna, Reunion Island, and Zugspitze) have a strong negative percent difference (nearing $100 \%)$ between observing platforms and NDACC's total columns without applying the crosskernel calculations. Mauna Loa has a strong positive percent difference (over 50\%) against every platform as well, apart from GEOS-Chem's small positive percent difference. A common factor between these stations is their large variation in elevation within the $200 \mathrm{~km}$ radius. Each of these NDACC stations are on a high elevation zone $(1500-3000 \mathrm{~km})$ except for Kiruna, which is within $200 \mathrm{~km}$ of a high elevation zone (Center for International Earth Science Information \& Network(CIESIN)/Columbia University, 2021). Since these percent differences are not based on cross-kernel calculation values, the large differences may stem from a priori assumptions and instrument biases discussed in Section 3.2.1. This is likely the case as these strong positive and negative percent differences are significantly reduced when the NDACC cross-kernel calculations are applied. Looking at the same six high elevation stations for the NDACC crosskernel comparison, the percent difference is significantly reduced as expected. 


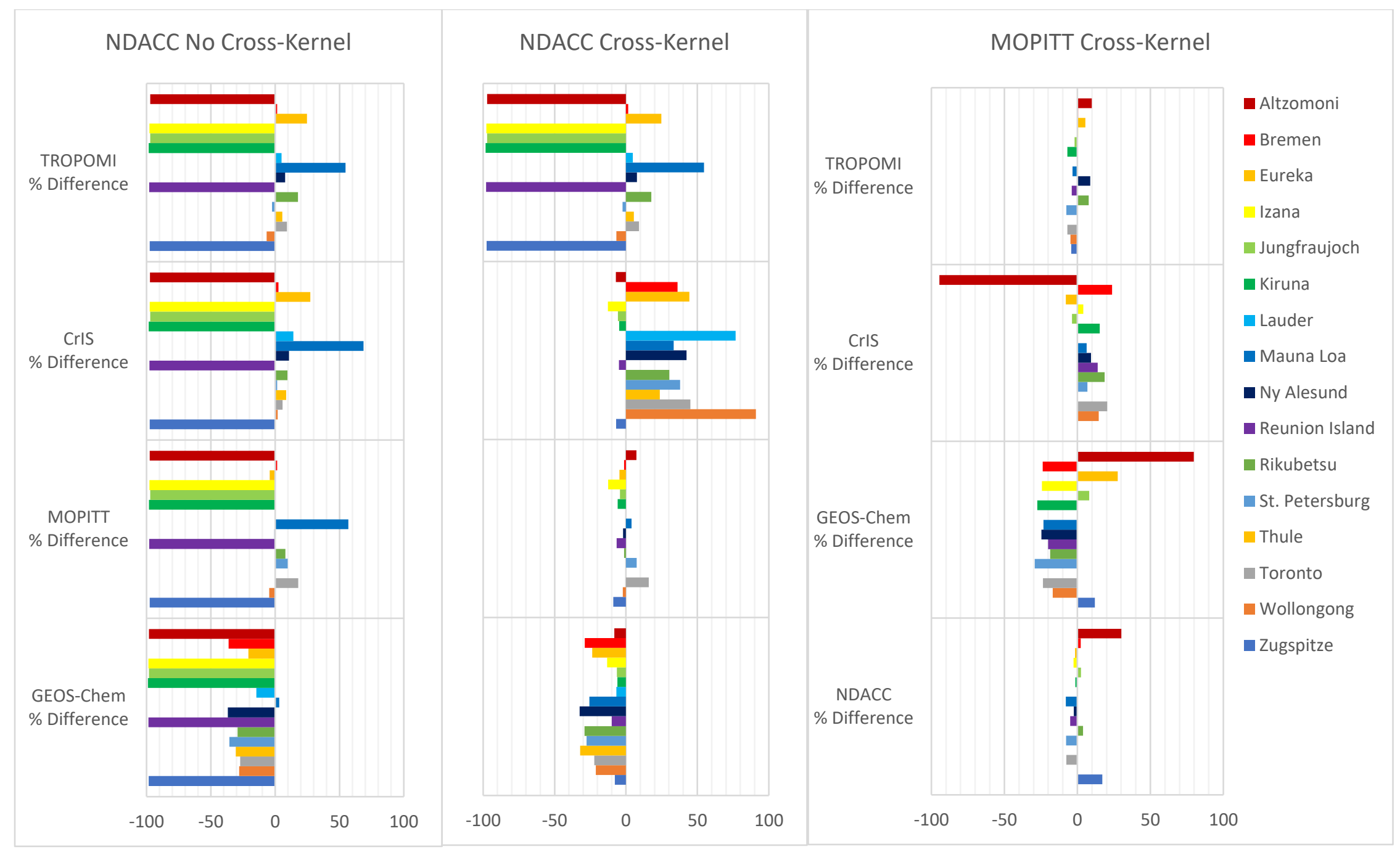

Figure 4.1.1.1: Four-month mean total column percent difference of each NDACC station location. (Left) Total column \% difference between observing platforms and NDACC stations when no cross-kernel calculation is applied. (Middle) Total column \% difference between observing platforms and NDACC total columns when the NDACC cross-kernel calculation is applied, except not applied to TROPOMI. (Right) Total column \% difference between observing platforms and MOPITT total columns when the MOPITT cross-kernel calculation is applied, except not applied to TROPOMI. 


\section{i) CrIS - NDACC Cross-Kernel}

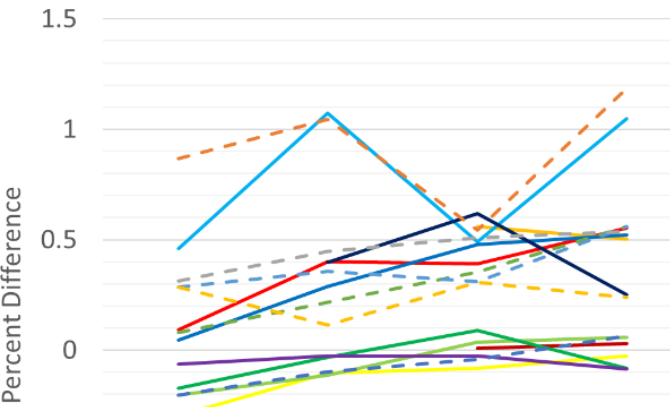

$-0.5$

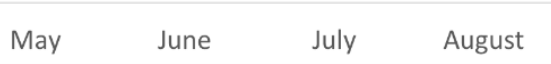

-Altzomoni

-Bremen

—Eureka

- Izana

—Jungfraujoch

- Kiruna

—Lauder

-Mauna Loa

- Ny Alesund

—Reunion Island

- - Rikubetsu

- -St. Petersburg

- -Thule

- - Toronto

- -Wollongong

- -Zugspitze ii) MOPITT - NDACC Cross-Kernel

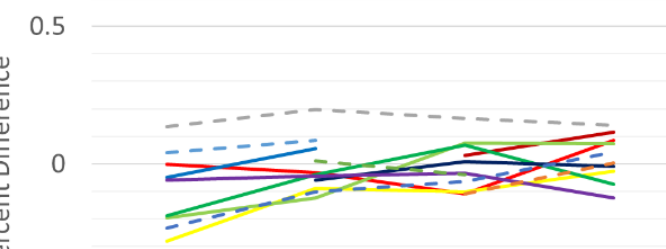

$-0.5$ -Altzomoni -Bremen -Eureka - Izana —Jungfraujoch -Kiruna - Lauder -Mauna Loa -Ny Alesund

- Reunion Island - -Rikubetsu

- St. Petersburg

-- Thule

- -Toronto

- -Wollongong

$-1$

May June July August - -Zugspitze iii) GEOS-Chem - NDACC Cross-Kernel

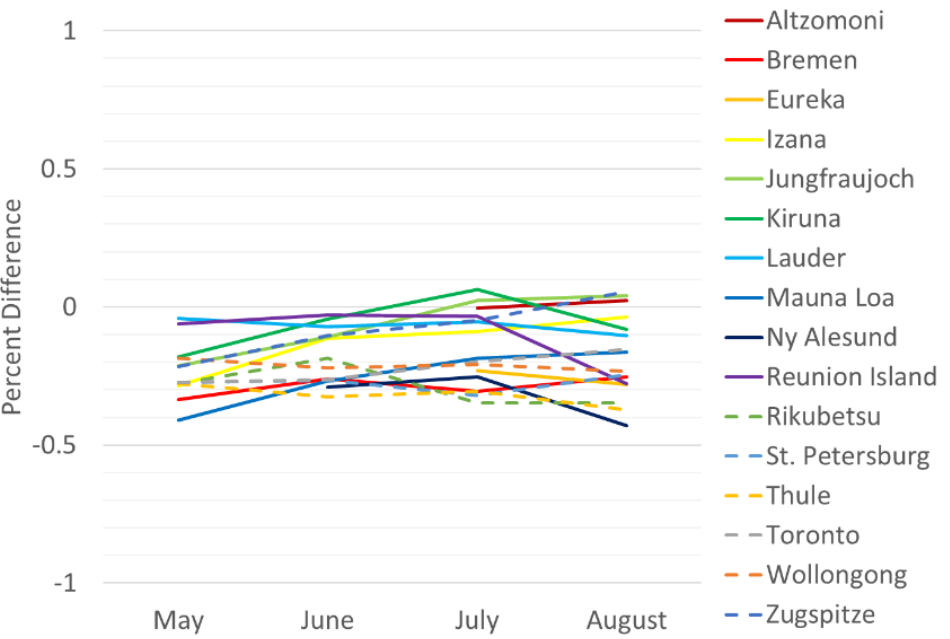

iv) TROPOMI - NDACC without Cross-Kernel

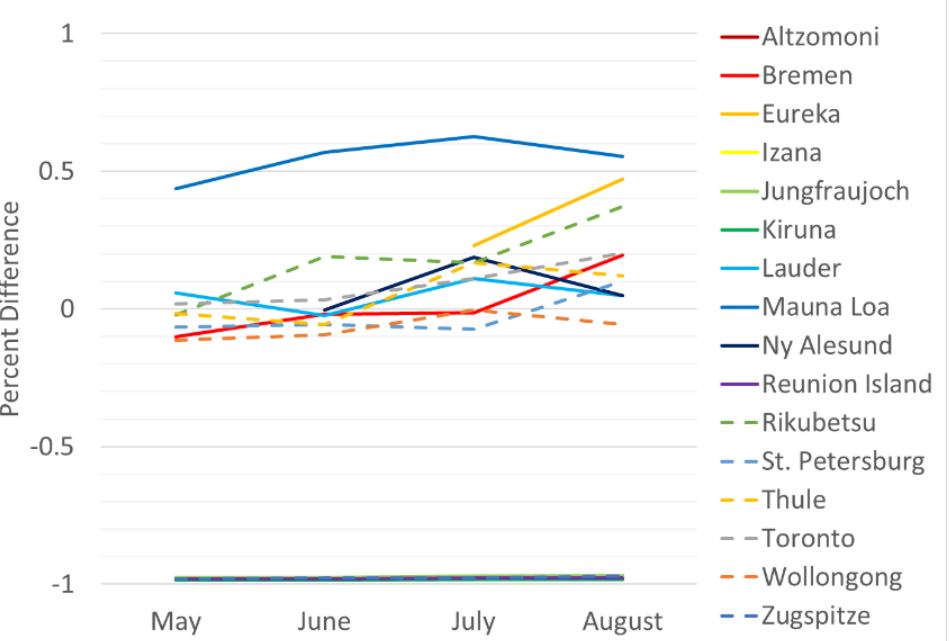

Figure 4.1.1.2: NDACC cross-kernel monthly mean total column percent difference trends against each observing platform. i) CrIS total column percent difference for NDACC crosskernel. ii) MOPITT total column percent difference for NDACC cross-kernel. iii) GEOS-Chem total column percent difference for NDACC cross-kernel. iv) TROPOMI total column percent difference against NDACC with no cross-kernel calculation applied. 


\section{i) CrIS - MOPITT Cross-Kernel}

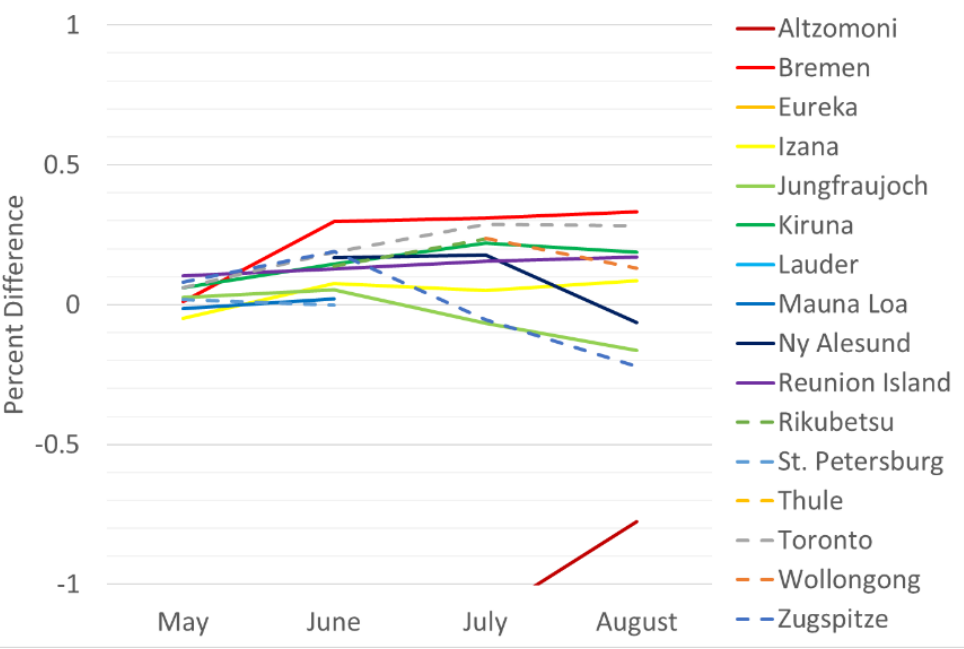

iii) NDACC - MOPITT Cross-Kernel

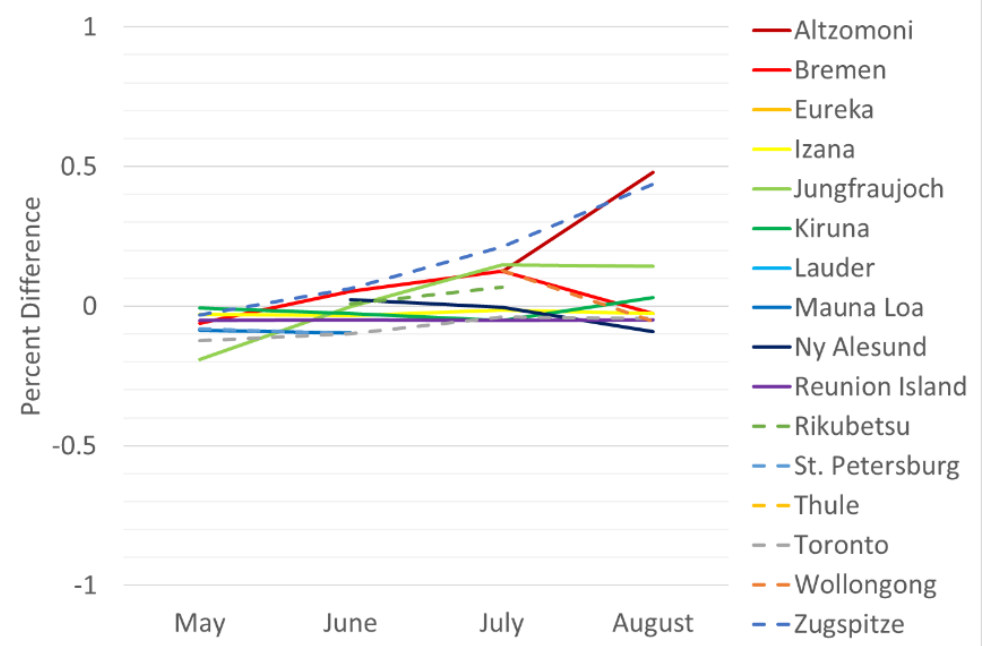

ii) GEOS-Chem - MOPITT Cross-Kernel

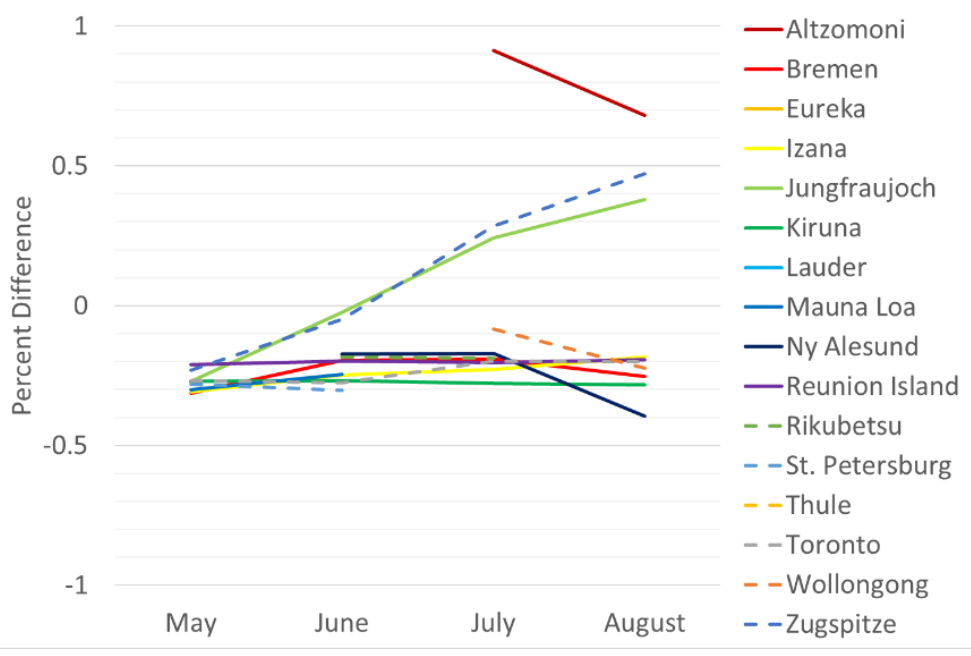

iv) TROPOMI - MOPITT without Cross-Kernel

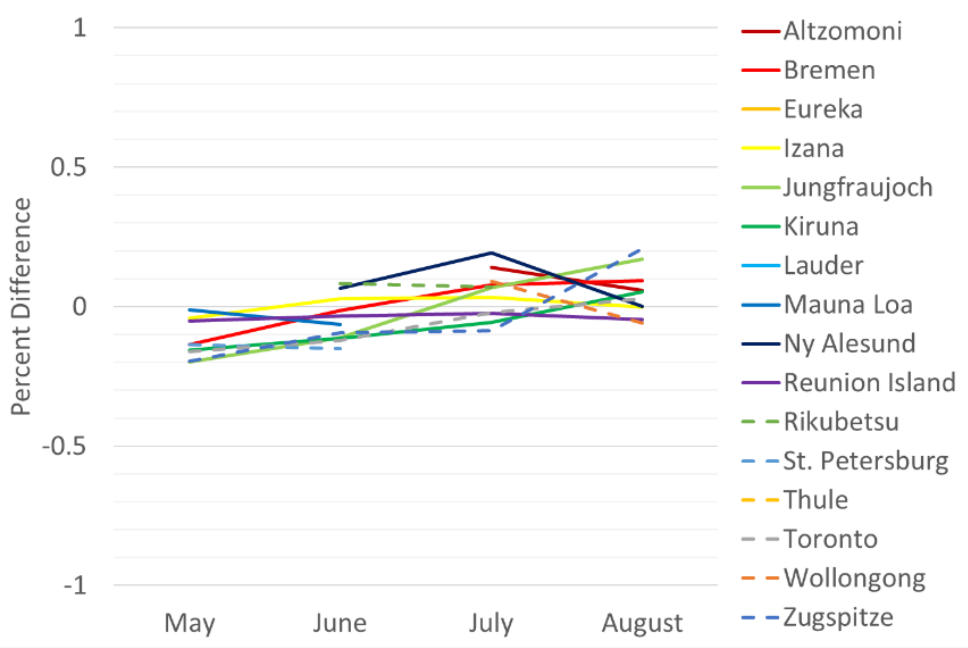

Figure 4.1.1.3: MOPITT cross-kernel monthly mean total column percent difference trends against each observing platform. i) CrIS total column percent difference for MOPITT cross-kernel. ii) GEOS-Chem total column percent difference for MOPITT cross-kernel. iii) NDACC total column percent difference for MOPITT cross-kernel. iv) TROPOMI total column percent difference against MOPITT with no cross-kernel calculation applied. 
The CrIS total column compared to NDACC cross-kernel (Figure 4.1.1.1, middle) has a strong overall average positive percent difference at $+26.16 \%$, where only the noted six stations have small negative percent differences. The physical location of these six NDACC stations at high elevation may contribute to their smaller percent difference. CrIS is more sensitive to CO in the free troposphere than in the boundary layer, where its sensitivity drops at pressures exceeding $700 \mathrm{hPa}$ (Smith et al., 2020b). The high elevations of these six NDACC stations minimise the number of points below $700 \mathrm{hPa}$, and therefore contribute to a lower percent difference. Again, Kiruna station itself is not located at a high elevation but has high elevations within $200 \mathrm{~km}$. Other NDACC stations also have high elevation zones within $200 \mathrm{~km}$, but do not share Kiruna's relationship with high elevation zones. Further in-depth investigation may be required, but it is also possible that the CrIS 2019 total columns coincidently align well with 2016 total columns.

Mauna Loa station is also located in a high elevation zone, but its percent difference is not significantly reduced like the other six stations. This may be attributed to the large elevation difference from the station location to the nearby surrounding sea, where the $200 \mathrm{~km}$ radius incorporates a large portion of sea level elevations compared to most other coastal/island stations. It may also be attributed to a larger difference in background CO loading, as Hawaii is not within close proximity to any continents like other island stations. The remaining stations have a relatively large average positive percent difference with Lauder and Wollongong above $+50 \%$.

The CrIS total column compared to the MOPITT cross-kernel (Figure 4.1.1.1, right) also has a strong but reduced average positive percent difference at $+1.95 \%$, weighted down by a 
strong negative percent difference for Altzomoni nearing $-100 \%$. The small percent difference with the MOPITT cross-kernel compared to the NDACC cross-kernel indicates that CrIS's total column observations closer match to MOPITT. This is reasonable as they both calculate CO using IR measurements from high orbit. CrIS's consistent positive percent difference across all station locations may be indicative of the CLIMCAPS algorithm overestimating boundary layer CO concentrations (see Section 4.1.2).

Both GEOS-Chem total column cross-kernel comparisons have strong overall average negative percent differences: $-18.81 \%$ for the NDACC cross-kernel, and $-7.44 \%$ for the MOPITT cross-kernel. The reduction in percent difference against the MOPITT cross-kernel is weighted by a strong positive percent difference for Altzomoni at $+79.71 \%$, followed by positive percent differences for Eureka, Jungfraujoch and Zugspitze. The overall large negative percent difference for the NDACC cross-kernel may be due to the large difference in area observed. The NDACC station measures a spatially specific column directly above the station, whereas the GEOS-Chem model returns a column average concentration over a gridded area of $2^{\circ} \times 2.5^{\circ}$ latitude-longitude. For example, where the gridded area is mostly rural, or marine compared to a single NDACC point location in an urban center then it is reasonable that the averaged GEOSChem total column is less than NDACC's.

The total column percent differences between MOPITT and NDACC stations are significantly smaller than the CrIS and GEOS-Chem comparisons. The MOPITT total column percent difference against the NDACC cross-kernel is $-1.06 \%$. The NDACC total column percent difference against the MOPITT cross-kernel is $+1.40 \%$, weighted with a comparatively strong positive percent difference for Altzomoni (30.16\%) and Zugspitze (+17.04\%). MOPITT and 
NDACC's smaller percent differences my be a result of their similar sensitivity and data range. NDACC is most sensitive from the surface to $20 \mathrm{~km}(\sim 50 \mathrm{hPa})$ (Langerock et al., 2018), and MOPITT only provides retrieval values from the surface to $100 \mathrm{hPa}$ (Deeter, MOPITT Algorithm Development Team, et al., 2017; Francis et al., 2017). Whereas CrIS and TROPOMI retrieval products span from the surface to TOA; CrIS has stronger sensitivity from 200 to $700 \mathrm{hPa}$ (Smith et al., 2020b), and TROPOMI's sensitivity is over the full column (D. Fu et al., 2016). The MOPITT and NDACC total columns are also from 2016 observations, whereas CrIS is from 2019. Since the observations are not all from the same year, it is not expected that all instrument measurements will be observing the same CO columns.

Interestingly, the TROPOMI average percent difference when compared to MOPITT (Figure 4.1.1.1, right) is minimal at $0.5 \%$ despite not using a cross-kernel comparison calculation. This may be indicative of TROPOMI's high agreement with MOPITT observations. However, due to TROPOMI's higher resolution, there are a greater number of sampling points incorporated in this comparison than MOPITT. This may introduce sampling bias, where the greater number of points averaged may be hiding TROPOMI's variability. This is also true for TROPOMI's comparison with NDACC, where the percent difference is significantly larger. NDACC has a much more selective view of the atmospheric state with a single point observation. It therefore is understandable that the differences are greater.

Looking at the monthly average trends for the NDACC cross-kernel and MOPITT crosskernel comparisons, some minor similarities are observed. In nearly all cross-kernel comparisons in Figures 4.1.1.2 and 4.1.1.3, the overall trendlines appear to have a slight positive slope from May to August, regardless of where the trendline is located with respect to 
$0 \%$. A positive slope indicates that the $\mathrm{CO}$ total column value of the observing platform is decreasing in comparison to the base instrument total columns, possibly indicating that each observing platform sees less of the total column by August. Greater CO loading positively impacts the DOFS of NIR instruments observing total columns (Deeter et al., 2015). In the Northern Hemisphere, CO loading (and thus DOFS) is generally greatest in April and lowest in September (Deeter et al., 2015) over large scale averages. Therefore, it is sensible that the NIR observing satellite instruments see less of the total column by August compared to NDACC (seen as the positive slope for all plots in Figure 4.1.1.2).

For the NDACC cross-kernel summer trend comparisons (Figure 4.1.1.2) with CrIS, there appears to be two groupings of trendlines where the lower group is composed of the six mentioned high elevation stations: Altzomoni, Izana, Jungfraujoch, Kiruna, Reunion Island, and Zugspitze. This group follows the overall positive slope with the percent difference generally closest to zero in July and August. The remaining stations also follow the overall positive slope, but with the percent difference generally closest to zero in May. The Lauder and Wollongong have highly variable percent difference in the Southern Hemisphere - although Reunion Island is also in the Southern Hemisphere and has low and stable percent difference. For the NDACC cross-kernel comparison with MOPITT, the station trendlines again appear to follow the general positive slope with the percent difference average closest to zero in July and August. For the NDACC cross-kernel comparison with GEOS-Chem, the positive slope is not as apparent as most stations' percent difference appear relatively stable throughout the four months.

For the MOPITT cross-kernel summer trend comparison (Figure 4.1.1.3) with CrIS, the general positive slope is observed again with the percent difference closest to zero in May. 
However, there is a noticeable divergence in percent difference between stations by August, with a uniquely large negative percent difference for Altzomoni. For the MOPITT cross-kernel comparison with GEOS-Chem, again the positive slope is not as apparent with most station values remaining relatively stable at a negative percent difference. The exceptions being Jungfraujoch and Zugspitze following a similar strong positive slope over the four months with values closest to zero in June, and Altzomoni standing alone with a high positive percent difference. For the MOPITT cross-kernel comparison with NDACC, the positive slope is still observed although weak. There is also a noticeable divergence in percent difference between stations by August, although weaker than the divergence seen in the CrIS comparison.

The TROPOMI percent difference comparison with both NDACC and MOPITT (without cross-kernel calculations) shows a positive slope trend over the four months - excluding the noted six outliers with NDACC. Again, the positive slope is likely indicative of greater TROPOMI DOFS in May compared to August due to CO loading (Deeter et al., 2015). As explained, this makes sense in comparison to NDACC. However, MOPITT is a NIR observing instrument as well. Here the positive slope may also be reflective of TROPOMI having even greater CO total column observing capability than MOPITT.

These CO total column observations show the relationship between the various observing platforms. Overall, the cross-kernel comparisons between 2016 NDACC and MOPITT have the lowest percent differences, and therefore the best total column agreement. 2019 TROPOMI observations also had a fair agreement with MOPITT observations despite not using cross-kernel calculations for comparison. 2019 CrIS and GEOS Chem observations have greater 
percent differences. However, this analysis is limited on its own. A profile analysis can reveal more information on where the column discrepancies lie, and at what altitudes.

\subsubsection{Profile comparison observations}

To further investigate $\mathrm{CO}$ total column differences between instruments, the shapes of the CO profiles are compared using RMSE. The RMSE is calculated over the full column's profile, a section between $100 \mathrm{hPa}$ and the surface, between $100 \mathrm{hPa}$ and the boundary layer (approximate free troposphere), and within in the boundary layer (BL). As noted in Section 3.2 the $100 \mathrm{hPa}$ limit is approximate, where the closest layer to $100 \mathrm{hPa}$ is selected rather than interpolating a value. Same for the boundary layer upper edge where the closest layer to 800 $\mathrm{hPa}$ is selected. Figures 4.1.2.1 through 4.1.2.3 show the four-month mean profile RMSE values of the above profile segments from left to right. The smaller the RMSE value, the closer the CO profile sections' shapes are to each other.

Note that there are absent values in certain graphs indicating where data did not meet observation requirements outlined in Section 3.2. However, some NDACC station locations are missing values for lower altitude sections only. This occurs when an NDACC station's elevation is significantly higher than the surface elevation measured by the satellite instruments. For example, Altzomoni's NDACC cross-kernel comparisons over the full profile is compared across all instruments over an altitude of 3985 metres above sea level (NDACC, 2021). The MOPITT and CrIS cross-kernel comparisons over the full profile RMSE only incorporates NDACC's profile values above 3985 metres. Since the NDACC station is not within the boundary layer, there are no Altzomoni values within the BL for cross-kernel comparisons with NDACC. See Appendix B.1 for a more illustrative example. 


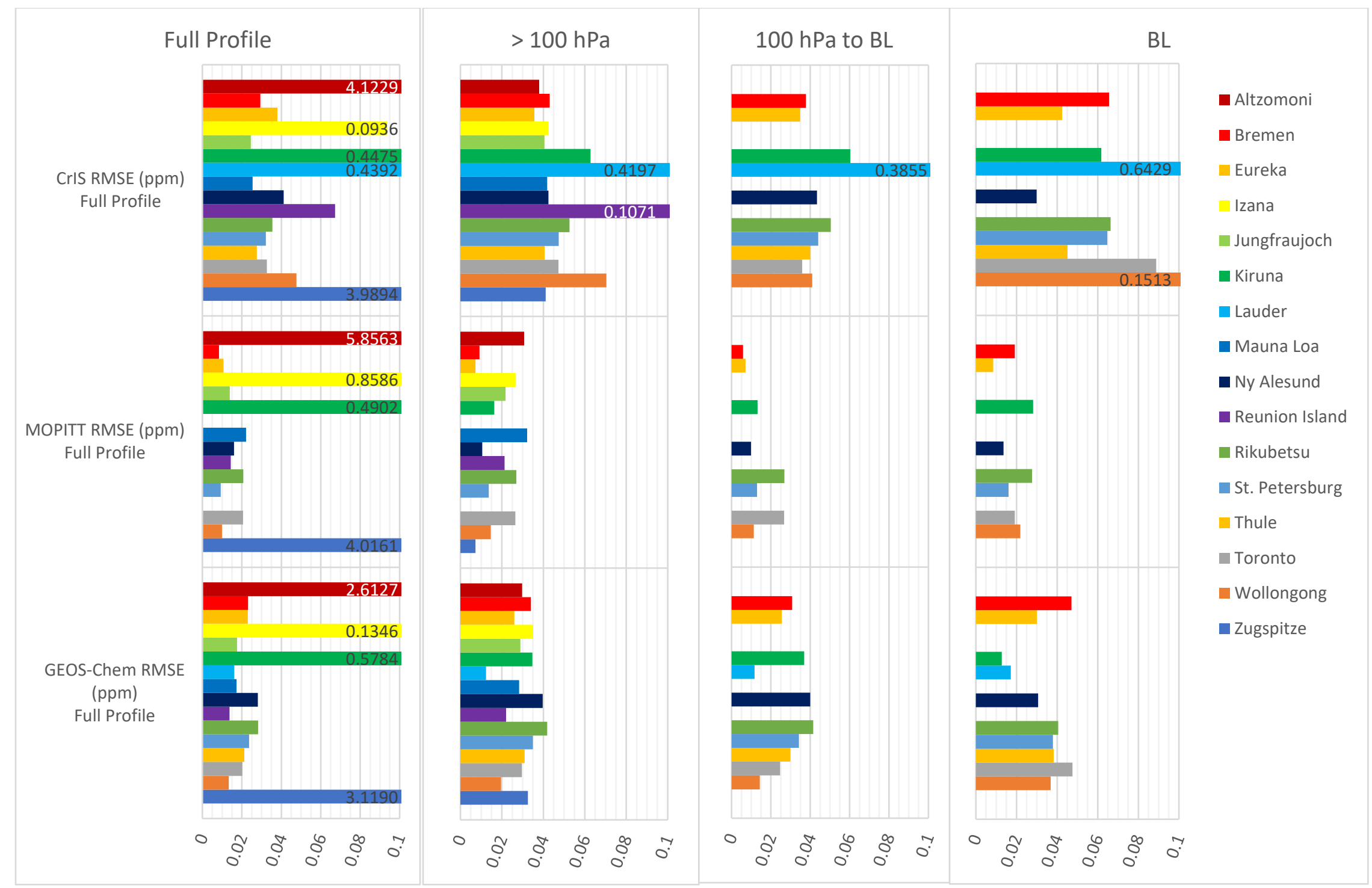

Figure 4.1.2.1: Four-month mean CO profile RMSE (ppm) for NDACC Cross-Kernel comparisons. Full profile and section profile comparisons are shown. Order from left to right are the RMSE comparisons for the full profile, $100 \mathrm{hPa}$ to the surface, $100 \mathrm{hPa}$ to the approximate top of the boundary layer (BL) at $\sim 800 \mathrm{hPa}$, and the values within the BL to the surface. 


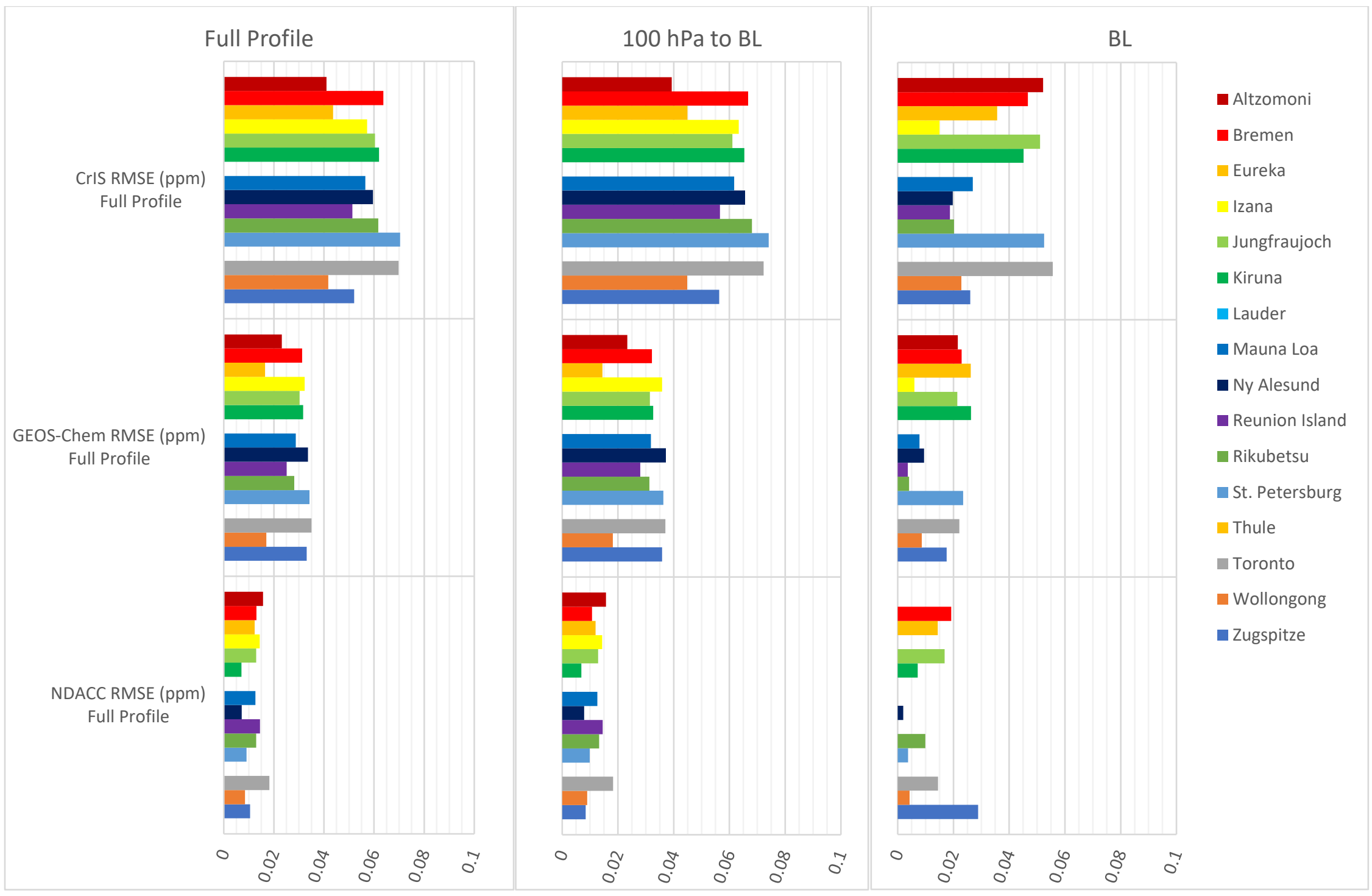

Figure 4.1.2.2: Four-month mean CO profile RMSE (ppm) for MOPITT Cross-Kernel comparisons. Full profile and section profile comparisons are shown. Order from left to right are the RMSE comparisons for the full profile, $100 \mathrm{hPa}$ to the approximate top of the boundary layer (BL) at $800 \mathrm{hPa}$, and the values within the BL to the surface. Note that the MOPITT full profile is the same as $\sim 100 \mathrm{hPa}$ to the surface. 


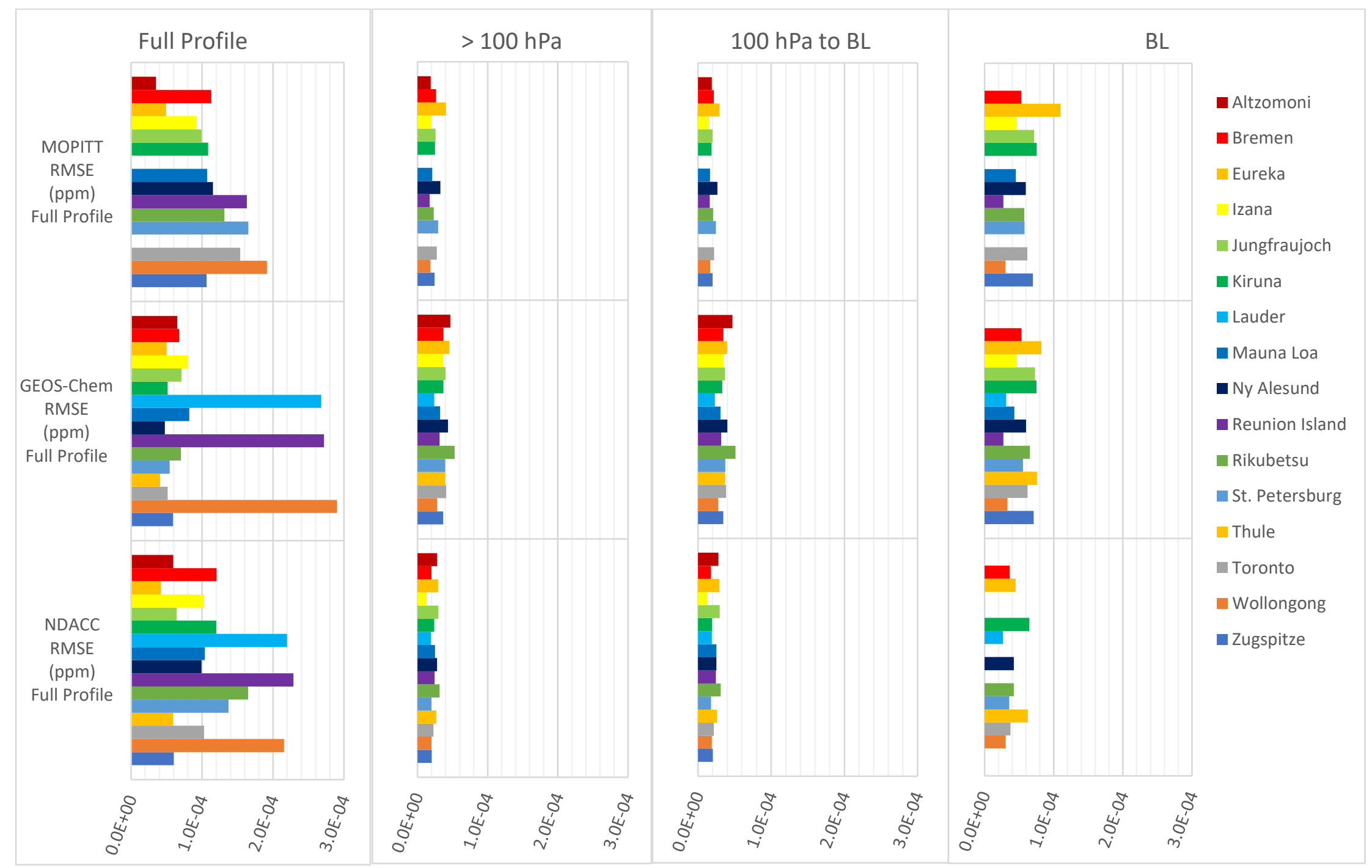

Figure 4.1.2.3: Four-month mean CO profile RMSE (ppm) for CrIS Cross-Kernel comparisons. Full profile and section profile comparisons are shown. Order from left to right are the RMSE comparisons for the full profile, $100 \mathrm{hPa}$ to the surface, $100 \mathrm{hPa}$ to the approximate top of the boundary layer (BL) at $800 \mathrm{hPa}$, and the values within the BL to the surface. 
Table 4.1.2.1: Total average four-month mean profile RMSE (ppm) of all stations across all cross-kernel calculations between instruments. Note that the NDACC cross-kernel average values include a column where all station values are averaged, and a column where the outliers are removed from the average.

\begin{tabular}{|c|c|c|c|c|c|}
\hline & & & \multicolumn{3}{|c|}{ Cross-Kernel Calculation } \\
\hline & & & NDACC & MOPITT & CrIS \\
\hline \multirow{16}{*}{ 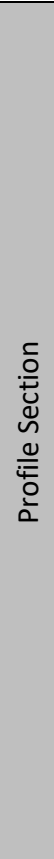 } & \multirow{4}{*}{ Full Profile } & NDACC & & $1.21 \mathrm{E}-02$ & 1.19E-04 \\
\hline & & GEOS-Chem & $4.18 \mathrm{E}-01$ & $2.86 \mathrm{E}-02$ & $1.01 \mathrm{E}-04$ \\
\hline & & MOPITT & 8.12E-01 & & 1.16E-04 \\
\hline & & CrIS & 5.93E-01 & $5.65 \mathrm{E}-02$ & \\
\hline & \multirow{4}{*}{$>100 \mathrm{hPa}$} & NDACC & & $1.21 \mathrm{E}-02$ & $2.38 \mathrm{E}-05$ \\
\hline & & GEOS-Chem & $3.00 \mathrm{E}-02$ & $2.86 \mathrm{E}-02$ & $3.82 \mathrm{E}-05$ \\
\hline & & MOPITT & $1.89 \mathrm{E}-02$ & & $2.50 \mathrm{E}-05$ \\
\hline & & CrIS & 7.33E-02 & $5.65 \mathrm{E}-02$ & \\
\hline & \multirow{4}{*}{$100 \mathrm{hPa}$ to $\mathrm{BL}$} & NDACC & & $1.19 \mathrm{E}-02$ & $2.28 \mathrm{E}-05$ \\
\hline & & GEOS-Chem & $2.90 \mathrm{E}-02$ & $3.04 \mathrm{E}-02$ & $3.61 \mathrm{E}-05$ \\
\hline & & MOPITT & $1.42 \mathrm{E}-02$ & & 2.04E-05 \\
\hline & & CrIS & 7.73E-02 & $6.00 \mathrm{E}-02$ & \\
\hline & \multirow{4}{*}{$\mathrm{BL}$} & NDACC & & $1.21 \mathrm{E}-02$ & 4.23E-05 \\
\hline & & GEOS-Chem & $3.39 \mathrm{E}-02$ & $1.58 \mathrm{E}-02$ & 5.69E-05 \\
\hline & & MOPITT & $1.93 \mathrm{E}-02$ & & $5.88 \mathrm{E}-05$ \\
\hline & & CrIS & $1.26 \mathrm{E}-01$ & $3.49 E-02$ & \\
\hline
\end{tabular}

Figure 4.1.2.1 shows the four-month average NDACC cross-kernel RMSE comparisons for each NDACC station over each profile section. All values for the full profile are below 0.08 with the exception of five stations: Altzomoni, Izana, Kiruna, Lauder and Zugspitze. Four of these stations (Altzomoni, Izana, Kiruna, and Zugspitze) have a dramatic decrease in RMSE for all instruments when looking at the profile section between $100 \mathrm{hPa}$ and the surface, indicating that their profile shapes deviate from NDACC's at higher altitudes above $100 \mathrm{hPa}$. Remember, $\mathrm{CO}$ at high altitudes above $100 \mathrm{hPa}$ are significantly lower than below. Therefore, small differences in $\mathrm{CO}$ at high altitudes would have a greater impact on RMSE. Lauder only has a high RMSE for the full profile CrIS comparison and is consistently high RMSE across all CrIS profile 
sections. This indicates that the CrIS comparison with the NDACC cross-kernel for Lauder does not well represent the NDACC profile over the course of these four months.

For the remaining stations, all full profile RMSE values are generally smaller than their respective $100 \mathrm{hPa}$ to surface RMSE values. This indicates that their upper atmosphere profile shape fits NDACC's well above $100 \mathrm{hPa}$. When comparing RMSE values between the free troposphere and the BL, the average CrIS, MOPITT, and GEOS-Chem RMSE values are mostly larger within the BL profile section (Table 4.1.2.1). Of the observing platforms, CrIS has the largest average RMSE values for all profile sections, and MOPITT has the smallest. It is reasonable that the CrIS profile sections' RMSE values are largest in the NDACC cross-kernel comparisons as NDACC's CO sensitivity is greatest at the surface and CrIS's sensitivity is poor below $700 \mathrm{hPa}$ (Smith et al., 2020b).

Figure 4.1.2.2 shows the four-month average MOPITT cross-kernel RMSE comparisons for each NDACC station over each profile section. Note that the full profile section for MOPITT is the same as $100 \mathrm{hPa}$ to the surface. In this Figure, the largest RMSE values are in the free troposphere section between $100 \mathrm{hPa}$ and the $\mathrm{BL}$ for the CrIS and GEOS-Chem observing platforms (Table 4.1.2.1), indicating greatest disagreement in the free troposphere when compared with MOPITT. This differs from the other profile cross-kernel comparisons where agreement is strongest within the free troposphere. The largest RMSE for NDACC compared to the MOPITT cross-kernel is within the boundary layer (Table 4.1.2.1), which is sensible as this is where NDACC's sensitivity is greatest and MOPITT's sensitivity is lowest. Of the observing platforms, CrIS has the largest average RMSE values for all profile sections, and NDACC has the smallest. This may be a result of NDACC having a higher profile DOFS and therefore better 
sensitivity to the profile shape than CrIS, which has a lower profile DOFS than MOPITT (see Section 4.1.3).

Figure 4.1.2.3 shows the four-month average CrIS cross-kernel RMSE comparisons for each NDACC station over each profile section. Here, the full profile RMSE values are largest, indicating a poor fit at altitudes greater than $100 \mathrm{hPa}$ across all observing platforms. All observing platforms' average RMSE values are also larger in the BL section compared to the free troposphere section (Table 4.1.2.1), likely a result of CrIS's lower sensitivity below $700 \mathrm{hPa}$ (Smith et al., 2020b). The NDACC and MOPITT comparisons with the CrIS cross-kernel have similar average RMSE values within each profile section except the BL. It should also be noted that all RMSE values in the CrIS cross-kernel comparisons are smaller than the cross-kernel comparisons of NDACC and MOPITT, where all CrIS cross-kernel values are below 0.0003 compared to most being below 0.08 respectively.

Figures 4.1.2.4 through 4.1.2.6 (and B.2.1 though B.2.8) show the monthly average cross-kernel comparisons between each NDACC station for each observing platform. Like in Section 4.1.1, these figures are intended to reveal trends and relationships between stations over the course of the summer. Figure 4.1.2.4 (and B.2.1 to B.2.3) shows the monthly average RMSE values for the NDACC cross-kernels, Figure 4.1.2.5 (and B.2.4 to B.2.5) for the MOPITT cross-kernels, and Figure 4.1.2.6 (and B.2.6 to B.2.8) for the CrIS cross-kernels. In each Figure legend, black bolded station names indicate that the station has RMSE values off the chart. The grey bolded station names indicate that there are no RMSE values for that station. 
Of the monthly NDACC cross-kernel comparisons (Figures 4.1.2.4 and B.2.1 to B.2.3), average CrIS RMSE values appear to have the greatest variability between stations compared to MOPITT and GEOS-Chem. Excluding the outlying station values, all CrIS RMSE values also appear to have an overall negative slope from May to August across all profile sections except within the BL. A negative slope indicates that the overall fit with NDACC improved over the four months. An improved profile RMSE by August may be a result of increasing thermal contrast for stations within the Northern Hemisphere. TIR observing instrument DOFS improve with thermal contrast (Deeter et al., 2015), and therefore a lower RMSE is expected in summer months. Conversely, Wollongong and Ny Alesund RMSE values do not follow the negative slope trend and instead increase by August, indicating a degradation in fit.

For the BL (Figure B.2.3), the CrIS RMSE values are generally larger and have greater variability between stations. Again, this is reasonable as NDACC's CO sensitivity is highest at the surface and CrIS's sensitivity is poor below $700 \mathrm{hPa}$ (Smith et al., 2020b). Comparatively, the MOPITT and GEOS-Chem monthly average values have less variability between stations, and do not exhibit a noticeable overall slope over the four months. Like the CrIS comparison, Ny Alesund appears again to depart from station trends with increased RMSE values by August in the GEOS-Chem comparisons, but not within the BL. Within the GEOS-Chem BL, the RMSE values appear to converge slightly towards August. 
i) CrIS $100 \mathrm{hPa}$ to BL, NDACC Cross-Kernel

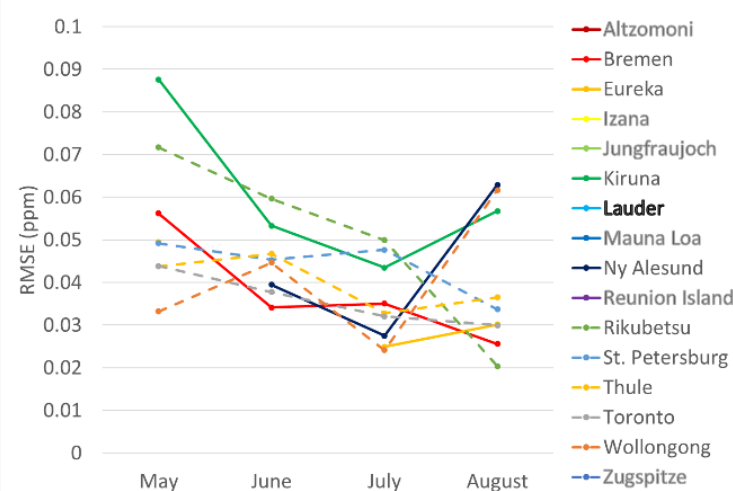

ii) MOPITT $100 \mathrm{hPa}$ to BL, NDACC Cross-Kernel

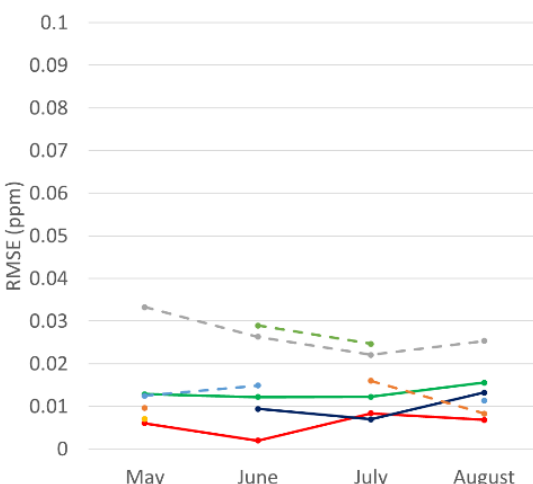

iii) GEOS-Chem 100 hPa to BL, NDACC Cross-Kernel

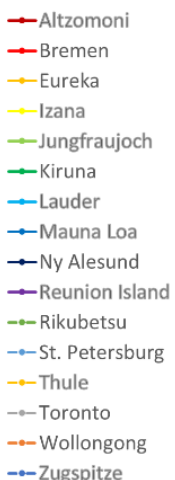

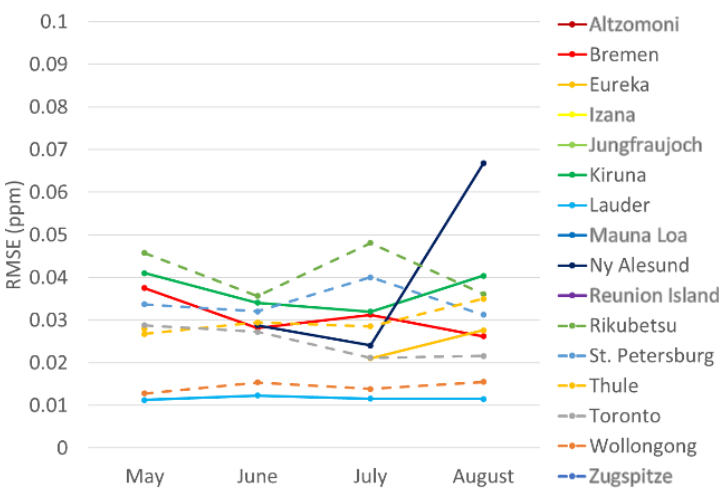

Figure 4.1.2.4: NDACC cross-kernel monthly mean profile RMSE (ppm) trends against each observing platform from $100 \mathrm{hPa}$ to the approximate top of the boundary layer (BL). i) CrIS profile RMSE for NDACC cross-kernel from $100 \mathrm{hPa}$ to the BL. ii) MOPITT profile RMSE for NDACC cross-kernel from $100 \mathrm{hPa}$ to the BL. iii) GEOS-Chem profile RMSE for NDACC cross-kernel from $100 \mathrm{hPa}$ to the BL. Stations bolded black in the legend have values off the chart. Stations bolded grey in the legend have no values over the full four months.
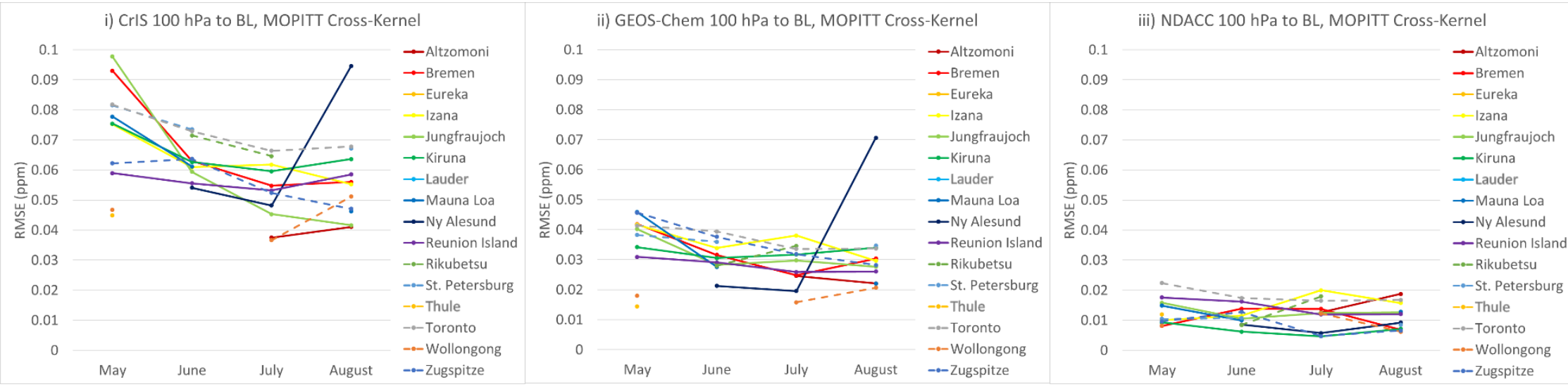

Figure 4.1.2.5: MOPITT cross-kernel monthly mean profile RMSE (ppm) trends against each observing platform from $100 \mathrm{hPa}$ to the approximate top of the boundary layer (BL). $i$ ) CrIS profile RMSE for MOPITT cross-kernel from $100 \mathrm{hPa}$ to the BL. ii) GEOS-Chem profile RMSE for MOPITT cross-kernel from $100 \mathrm{hPa}$ to the BL. iii) NDACC profile RMSE for MOPITT cross-kernel from $100 \mathrm{hPa}$ to the BL. Stations bolded black in the legend have values off the chart. Stations bolded grey in the legend have no values over the four months. 


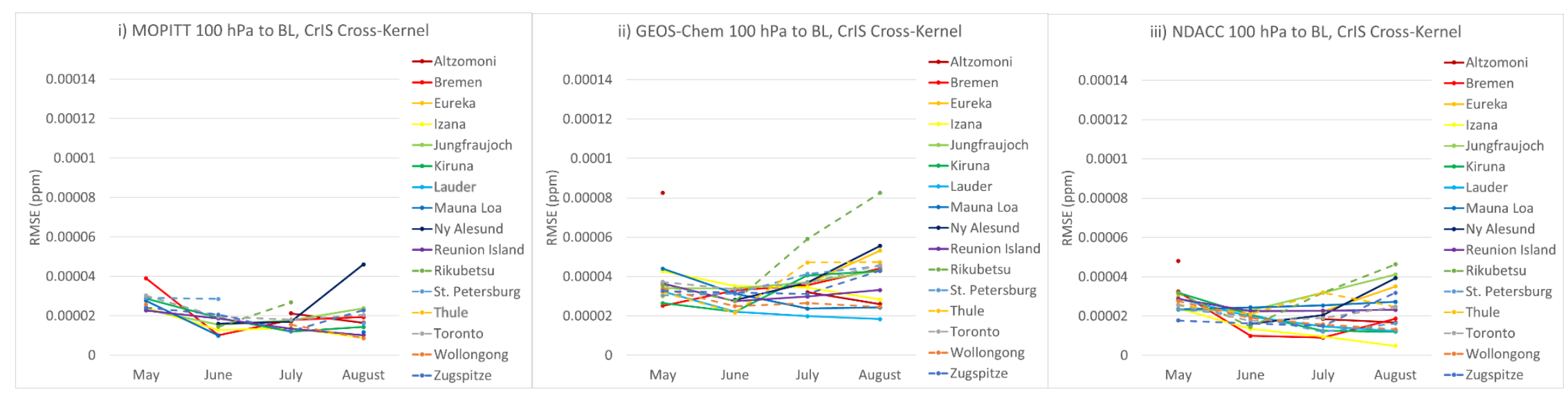

Figure 4.1.2.6: CrIS cross-kernel monthly mean profile RMSE (ppm) trends against each observing platform from 100 hPa to the approximate top of the boundary layer (BL). i) MOPITT profile RMSE for CrIS cross-kernel from $100 \mathrm{hPa}$ to the BL. ii) GEOS-Chem profile RMSE for CrIS cross-kernel from $100 \mathrm{hPa}$ to the BL. iii) NDACC profile RMSE for CrIS crosskernel from $100 \mathrm{hPa}$ to the BL. Stations bolded black in the legend have values off the chart. Stations bolded grey in the legend have no values over the four months. 
There are similar trends observed for the monthly MOPITT cross-kernel comparisons (Figures 4.1.2.5, B.2.4, and B.2.5). Again, the CrIS RMSE values appear to have the greatest variability between stations, highest variability within the $\mathrm{BL}$, and an overall negative slope from May to August. Ny Alesund also appears to deviate from the trends in both the CrIS and GEOS-Chem comparisons in August, except within the BL. Different from the NDACC crosskernel comparisons, the MOPITT cross-kernel comparison with GEOS-Chem also appears to have an overall negative slope from May to August in the full profile and free troposphere sections. The GEOS-Chem RMSE also appears to have greater variability between stations within the BL compared to the free troposphere. The MOPITT cross-kernel comparisons with NDACC appears to have the least variability between stations and does not exhibit a noticeable overall slope over the four months. Although it appears that within the NDACC BL, there is a larger variability between stations by August compared to May. Larger variabilities in August may be reflective of NDACC's selective view compared to averaged values over the $200 \mathrm{~km}$ radius.

For the monthly CrIS cross-kernel comparisons (Figures 4.1.2.6, and B.2.6 to B.2.8), all MOPITT RMSE profile section trendlines follow a negative slope with the steepest slopes over the full profile and within the BL. A steeper slope may be indicative of greater sensitivity to thermal contrast. Again, Ny Alesund appears to deviate from this trend for all CrIS profile sections except within the boundary layer (Figure B.2.8). For the GEOS-Chem full profile comparison, there appears to be two groupings of trendlines. Lauder, Reunion Island and Wollongong all have higher RMSE values and follow a negative slope over the four months. These three stations are also the only NDACC station analyses in the Southern Hemisphere. The 
remaining stations follow a positive slope over the four months in the Northern Hemisphere. This demonstrates a clear hemispherical difference within the GEOS-Chem model.

The GEOS-Chem to CrIS cross-kernel comparison for the $100 \mathrm{hPa}$ to surface and free troposphere sections do not exhibit a strong slope through the months, but they do show a strong increase in variability between stations by August compared to May. The GEOS-Chem RMSE values within the $B L$ show an overall negative slope over the months, with greater variability between stations in May compared to above the BL. The larger variabilities may be reflective of CrIS 2019 profiles differing from modeled 2016 norms. For the NDACC comparison, both the full profile and $B L$ sections have overall negative slopes through the months, and greater variability between stations than the $100 \mathrm{hPa}$ to surface and free troposphere sections. The $100 \mathrm{hPa}$ to surface and free troposphere sections also appear to have slightly greater variability between stations by August. Like the GEOS-Chem comparison, the NDACC comparison in the BL exhibits a negative slope with greater variability in May compared to the free troposphere. Again, greater variabilities with NDACC station observations may be reflective of NDACC's selective atmospheric view.

Overall, profile RMSE between platforms reveal best agreement between platforms within the free troposphere and lowest agreement in the upper atmosphere for NDACC crosskernel and CrIS cross-kernel comparisons. Differing from the other cross-kernels, the MOPITT cross-kernel has lowest agreement with CrIS and GEOS-chem within the free troposphere, and lowest agreement with NDACC within the boundary layer. Over the four months, monthly comparisons reveal greater agreement between platforms by August, possibly indicative of 
greater profile sensitivity due to increasing thermal contrast (Deeter et al., 2015). To investigate further, the next section compares the DOFS between instruments over each NDACC station.

\subsubsection{DOFS comparison observations}

The DOFS indicates the number of layers that the instrument is sensitive to in the vertical profile (Smith et al., 2020b). The lower the DOFS, the less information the vertical CO profile contains about its shape (Deeter et al., 2015). This section evaluates the average daily DOFS values between each instrument temporally and geographically. Figures 4.1.3.1 is time series graphs of the profile DOFS of each observing platform, averaged across all NDACC stations. Figure 4.1.3.2 is a boxplot representing the profile distributions of daily average DOFS for each observing platform for each NDACC station.

The averaged daily profile DOFS of each observing platform (Figure 4.1.3.1) appear relatively stable from May to August, with minimal trendline slopes. The NDACC DOFS trendline shows a slight negative slope of -0.0005 , indicating an averaged greater sensitivity at the start of May. However, this slope is not statistically significant when compared to its fitting error (0.00057). Differing from NDACC, the daily average TROPOMI, MOPITT, and CrIS DOFS all share the same slight positive slope of about 0.0003 , indicating an averaged increasing CO profile sensitivity by the end of August. An averaged increasing sensitivity is logical for TIR observing instruments as thermal contrast increases DOFS values seasonally due to insolation (Deeter et al., 2015). This however does not align with TROPOMI, a NIR observing instrument were DOFS is expected to be larger towards April, when the Northern Hemisphere's CO loading is largest (Deeter et al., 2015). Although, it should be noted that the TROPOMI slope is also not statistically significant when compared to its fitting error (0.00038). This may explain why the 
TROPOMI DOFS exhibits this trend. There is limited information available on the TROPOMI profile averaging kernels. It is only known that they are used to determine the total column concentrations (Landgraf et al., 2018).

The TROPOMI instrument has the highest average four-month profile DOFS at 7.65. The NDACC profile DOFS values have the greatest variability, likely attributed by its selective singular CO profile used in this study rather than an averaged DOFS over the $200 \mathrm{~km}$ radius. The NDACC profiles also have second highest average DOFS at 2.50. This is higher than the 2.22 mean observed by Buchholz et al. (2017) but is within their NDACC range of 1.65 to 2.92. A higher mean NDACC DOFS is likely a result of this study being over the summer months in the Northern Hemisphere, and most stations being located within the Northern Hemisphere. More available daylight may allow for more clear sky measurements at these stations, increasing the mean DOFS compared to winter months.

The average MOPITT profile DOFS is 1.42 , which is within the 0.76 to 1.72 range observed by Buchholz et al. (2017) but higher than the 1.32 mean. As thermal contrast increases DOFS values seasonally due to insolation (Deeter et al., 2015), it is reasonable that the mean is higher as most MOPITT observation comparisons in this study are in the Northern Hemisphere's summer months. CrIS DOFS values have the lowest variability and lowest average DOFS at 0.92, and therefore has the least information on the profile shape of the four instruments between May and August.

Figure 4.1.3.2 shows the distribution of daily average total column DOFS values between stations for each instrument. The NDACC profile DOFS values have the second greatest 
variability between stations with Altzomoni having its highest average DOFS, Bremen having its lowest average DOFS, and Mauna Loa having the largest DOFS inter-quartile range. MOPITT's profile DOFS values between stations are less variable with values over Eureka having the lowest average DOFS, followed by Ny Alesund. CrIS's profile DOFS values have the smallest interquartile ranges on average and are the least variable between stations with values over Altzomoni having the lowest average DOFS. TROPOMI's profile DOFS values are the largest and have relatively large inter-quartile ranges, although with little variability between the stations compared to NDACC.

The average DOFS values between observing platforms are possibly affected by their distance from the troposphere, our CO observational range of interest. It is reasonable that NDACC has the highest average DOFS as the observing instruments are located at the surface. It is also reasonable that CrIS has the lowest average DOFS it uses spectrometry compared to MOPITT's more precise gas correlation. However, TROPOMI also uses spectrometry and has a significantly greater profile DOFS. Little information is currently available on the TROPOMI profile DOFS, as it is a relatively new data product. TROPOMI profile retrievals and averaging kernels are not provided, with only a mention in its ATBD that the profile DOFS is used to determine the TROPOMI total column retrieval (Landgraf et al., 2018, p. 5).

The variability between daily average DOFS observations is likely affected by the number of observation points used in each station comparison. It is reasonable that NDACC DOFS values are the most variable as only a single daily measurement is used, and varying elevations of station locations would impact their sensitivity to the atmosphere above. MOPITT, CrIS, and TROPOMI have lower DOFS variability between stations as the troposphere is 


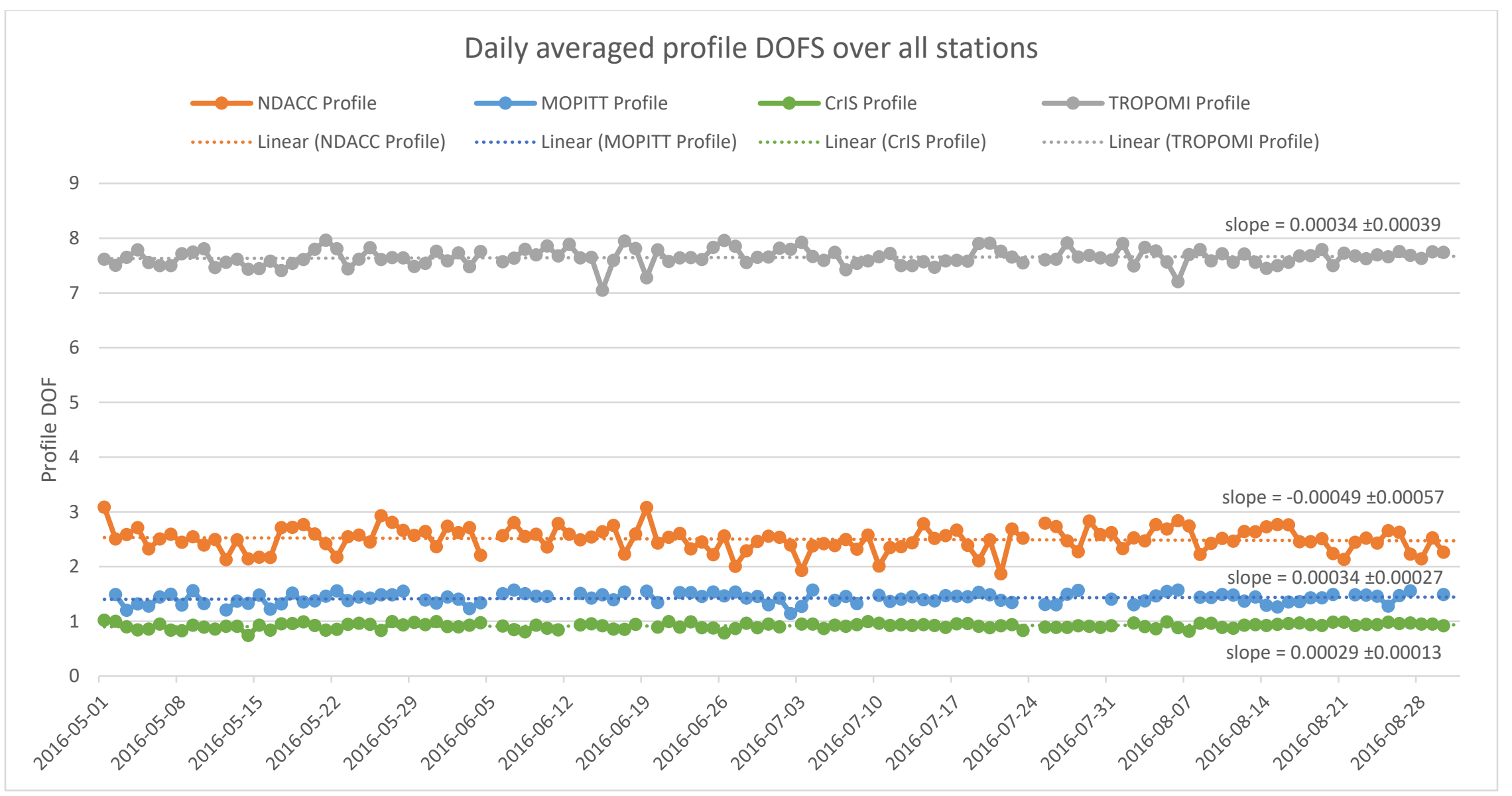

Figure 4.1.3.1: Daily profile DOFS of each observing platform, averaged over all stations. Note that the slopes for NDACC and TROPOMI are not statistically significant when compared to their slope error. 


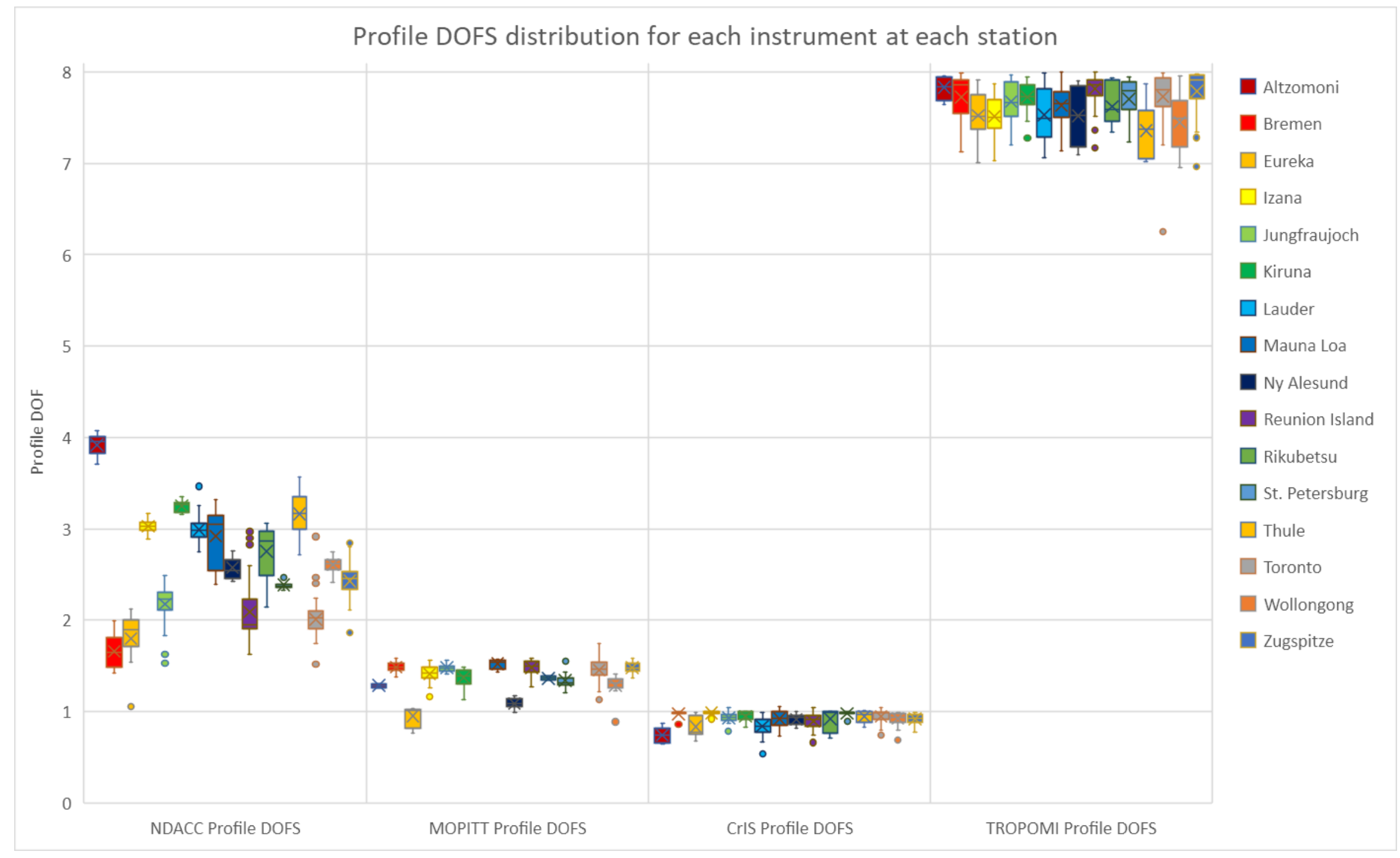

Figure 4.1.3.2: Distribution of average daily profile DOFS values for each observing platform over each NDACC station. 
observed from the same altitude across all stations. CrIS and TROPOMI likely have the smallest differences between stations as they have the most points averaged daily within the $200 \mathrm{~km}$ radius due to their high resolution.

Overall, TROPOMI appears to have the highest DOFS values followed by NDACC, MOPITT then CrIS. However, limited information TROPOMI's profile retrievals, averaging kernels, and DOFS makes it difficult to understand why TROPOMI's DOFS is significantly greater (Figure 4.1.3.2) and why its trendline is positive despite being a NIR observing instrument (Figure 4.1.3.1). Aside from TROPOMI, the remaining DOFS distributions and trends appear to follow expectations. The further the observing instrument is from the target the lower the DOFS, and for TIR observing instrument the DOFS increases slightly by the end of August due to insolation.

\subsubsection{Discussion}

This CO intercomparison study reveals that the MOPITT instrument best represents CO total columns and profiles compared to NDACC. The CrIS CLIMCAPS CO profile is comparable to MOPITT above the boundary layer and would be a suitable replacement product with a higher resolution should MOPITT be decommissioned. However, within the boundary layer CLIMCAPS tends to overestimate $\mathrm{CO}$. Although cross-kernel comparisons were not possible with the TROPOMI data products, the TROPOMI total column small percent difference with MOPITT without the cross-kernel calculations applied. Therefore, it is expected that TROPOMI would be a suitable replacement product with higher resolution for the CO total column. As proposed by Fu et al. (2016), a combination of the CrIS and TROPOMI CO retrievals has a potential for enhanced retrievals compared to the MOPITT NIR-TIR product. In this investigation, GEOSChem acted as a base intercomparison platform as the model does not rely on coinciding 
temporal and spatially limited observation retrievals. It also provides an expected typical CO profile and total column for 2016. Using GEOS-Chem as a base, the cross-kernel total column and profile evaluations are summarised in this section.

The GEOS-Chem average total column percent difference with the NDACC cross-kernel is $-18.81 \%$, with its absolute value greater than MOPITT's but smaller than CrIS's. As discussed in Section 4.1.1, the strong negative percent difference may be due to a large difference in area represented. Where NDACC represents a selective column above the station, and GEOS-Chem represents an average over a large gridded area. This also likely influences the profile analysis. The largest GEOS-Chem difference against the NDACC cross-kernel is within the full profile section (Table 4.1.2.1). This is reflective of NDACC's lower sensitivity to the upper atmosphere, increasing the profile retrieval differences at these altitudes. The second largest difference is within the BL, again likely due to the large difference in area represented where NDACC has a selective view. GEOS-Chem fits best with the NDACC cross-kernel within the free troposphere. Possibly influenced by greater weighting to the a priori due to lower NDACC sensitivity than the surface, which would be more reflective of typical modeled conditions.

Of the remaining NDACC cross-kernel comparisons, MOPITT has the best average total column percent difference (Figure 4.1.1.1) with an average percent difference closest to zero in August (Figure 4.1.1.2). The smaller percent difference by August may be attributed by the greater summer thermal contrast increasing the DOFS within the TIR channel (Deeter et al., 2015), as reflected in the Figure 4.1.3.1 increase in DOFS. MOPITT also has the lowest average profile RMSE with the NDACC cross-kernel for all profile sections below $100 \mathrm{hPa}$ (Table 4.1.2.1). In addition, MOPITT has the lowest RMSE across all CO profile sections in comparison with the 
NDACC cross-kernel. These observations are reflective of MOPITT's greater average profile DOFS than CrIS (Figure 4.1.3.1), MOPITT's greater representation of true profiles than GEOSChem, and CrIS having a declining sensitivity towards the surface (Smith et al., 2020b). When comparing monthly trendlines of the NDACC cross-kernel total column percent difference (Figure 4.1.1.2) against the profile RMSE trendlines (Figures 4.1.2.4, and B.2.1 to B.2.3), only Figure 4.1.1.2 exhibits a slight positive slope in percent difference over the four months. With best fit in August, this may be indicative of MOPITT NIR channel (total column sensitive channel) overestimating CO concentrations in May over the NDACC stations.

The CrIS to NDACC cross-kernel comparisons are comparable to MOPITT's, although only above the boundary layer (Figure 4.1.2.1). This is most evident in the NDACC cross-kernel total column comparison (Figure 4.1.1.1), where the six NDACC stations located at high altitudes above the boundary layer (Altzomoni, Izana, Jungfraujoch, Kiruna, Reunion Island, and Zugspitze) have comparable small percent differences, with percent difference closest to zero in August. Like MOPITT, the CrIS profile RMSE values for sections above the BL are also lowest in August. Again, due to an increase in thermal contrast strengthening the DOFS. Within the BL, CrIS has the largest RMSE compared to NDACC due to CrIS's lower sensitivity to elevations below 700 hPa (Smith et al., 2020b).

The GEOS-Chem average total column percent difference with the MOPITT cross-kernel is $-7.44 \%$, with its absolute value greater than both NDACC's and CrIS's. This larger percent difference may be reflective of GEOS-Chem representing expected CO profiles rather than true measured profiles by NDACC and CrIS. Comparing the average GEOS-Chem profile RMSE, its largest average RMSE value against the MOPTT cross-kernel is within the free troposphere and 
the lowest is within the boundary layer (Table 4.1.2.1). MOPITT's close boundary layer fit with GEOS-Chem may be indicative that MOPITT's retrieval reflects more typical expected CO emissions, relying more heavily on background a priori information than NDACC. Hence, the larger RMSE in the free troposphere may be reflective of greater MOPITT retrieval accuracy, straying from the GEOS-Chem predictive norm.

Of the MOPITT cross-kernel comparisons, NDACC has the lowest average total column percent difference (Figure 4.1.1.1) and lowest boundary layer RMSE value, complimenting the NDACC cross-kernel with MOPITT. The MOPITT cross-kernel profile comparison also has the largest average CrIS RMSE value within the free troposphere like GEOS-Chem (Table 4.1.2.1). Here, the meaning is difficult to determine. The greatest CrIS RMSE in the free troposphere compared to the MOPITT cross-kernel may be reflective of CrIS observing 2019 profiles rather than 2016, contributing to the large difference in their profile section of greatest sensitivity. The smaller CrIS average RMSE value in the boundary layer may be reflective of CLIMCAPS weighting the a priori heavier when approaching the surface, a closer match to MOPITT's surface a priori as discussed in the paragraph above when compared to GEOS-Chem. However, the average CrIS RMSE within the boundary layer is over twice as large as the GEOS-Chem RMSE for the MOPITT cross-kernel. Notably, the CrIS observations do improve outside the BL by August with increasing DOFS in comparison to the MOPITT cross-kernel (Figures 4.1.2.5, B.2.4, and B.2.5), demonstrating its increasing TIR channel sensitivity due to thermal contrast.

When comparing the MOPITT and NDACC profile RMSE values, the NDACC with MOPITT cross-kernel calculations yields smaller RMSE values across all profile sections than MOPITT with the NDACC cross-kernel (Table 4.1.2.1). This follows the statement made by Rodgers \& 
Connor (2003) where for cross-kernel comparison between instruments, a "higher resolution instrument would be expected to reproduce the lower resolution measurement better than the other way around." Here, NDACC is the higher resolution instrument and can better reproduce the lower MOPITT resolution profile when interpolated rather than the other way around. Notably the NDACC-MOPITT profile comparisons are similar for sections between the surface and $100 \mathrm{hPa}$. There is a greater difference between NDACC-MOPITT RMSE values for the full profile, which is reasonable as NDACC observations are not as sensitive to the upper atmosphere.

There is no total column CrIS cross-kernel comparison as a total column averaging kernel was not provided in the CLIMCAPS data product. Comparing the GEOS-Chem profile RMSE with the NDACC cross-kernel shows strongest agreement within the free troposphere, which may be a result of lower sensitivity below 700 hpa (Smith et al., 2020b) and differing GEOS-Chem model predictions in the upper atmosphere. Comparing the average CrIS RMSE between the MOPITT cross-kernel and NDACC cross-kernel, CrIS with MOPITT cross-kernel has lower average RMSE values for all profile sections except within the boundary layer (Table 4.1.2.1). This is likely a result of CrIS's low sensitivity to the boundary layer compared to NDACC, and therefore CrIS more closely represents the MOPITT retrieval. This is also reflected in the CrIS cross-kernel comparisons where the MOPITT BL RMSE value is greater than NDACC's.

Looking at all average CrIS cross-kernel RMSE values (all below 0.0003), they are significantly lower than the MOPITT cross-kernel and NDACC cross-kernel comparisons (below 0.08) (Table 4.1.2.1). A typical graphed profile view of CrIS cross-kernel comparisons show a very close fit along the profile from $100 \mathrm{hPa}$ to the boundary layer where the profiles deviate 
(Figure 4.1.4.1). This does not follow the Rodgers \& Connor (2003) noted earlier where a "higher resolution instrument would be expected to reproduce the lower resolution measurement better than the other way around." Instead, the observing platforms of lower vertical resolution than CrIS (less than 100 levels) are producing significantly smaller RMSE values with the CrIS cross-kernel calculation applied. The cause may be attributed to CrIS's comparatively lower DOFS. A lower DOFS weights the a priori CO profile heavier. Therefore, in the cross-kernel calculation the other observing instruments take on more of the CrIS a priori than the NDACC cross-kernel or MOPITT cross-kernel calculations. Hence, the significantly lower RMSE may be a result of the cross-kernel comparisons reflecting more of the CrIS a priori rather than their respective profile retrievals. CLIMCAPS's heavier weighting to the a priori may also be a result of systematic bias. It is a new product with limited validations studies and therefore less mature compared to an older instrument like MOPITT.
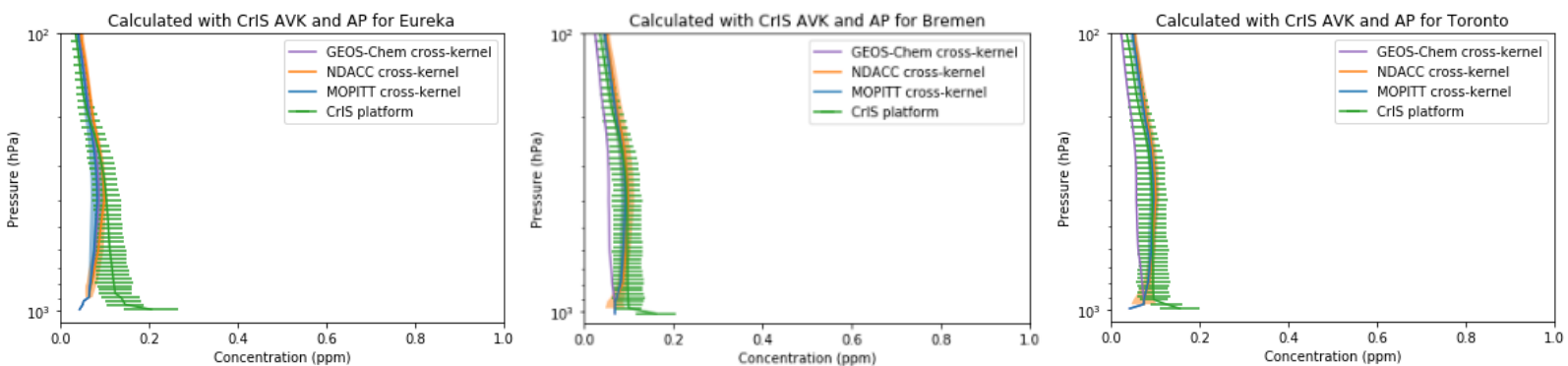

Figure 4.1.4.1: CO profile CrIS cross-kernel comparisons for Eureka on May 14 $4^{\text {th }}$, Bremen on June 23rd, and Toronto on July $20^{\text {th }}$. Note the profile deviation of every observing platform's CO concentration within the boundary layer.

The lowest CrIS cross-kernel RMSE values are within the free troposphere, this is reasonable as this is within CrIS' range of greatest sensitivity. The largest RMSE values are over the full profile. This larger RMSE indicates a larger difference in profile shape above $100 \mathrm{hPa}$. This difference is strongest for the GEOS-Chem and NDACC comparisons over Lauder, Ny 
Alesund, and Wollongong. In these instances, the CrIS cross-kernel calculation drops the other observing platforms' $\mathrm{CO}$ concentrations at high altitudes to zero (Figure 4.1.4.2). This occurs after the application of the CrIS averaging kernel, indicating that the 2019 CrIS retrieval is observing a significantly different profile above $100 \mathrm{hPa}$ than the other 2016 platforms.
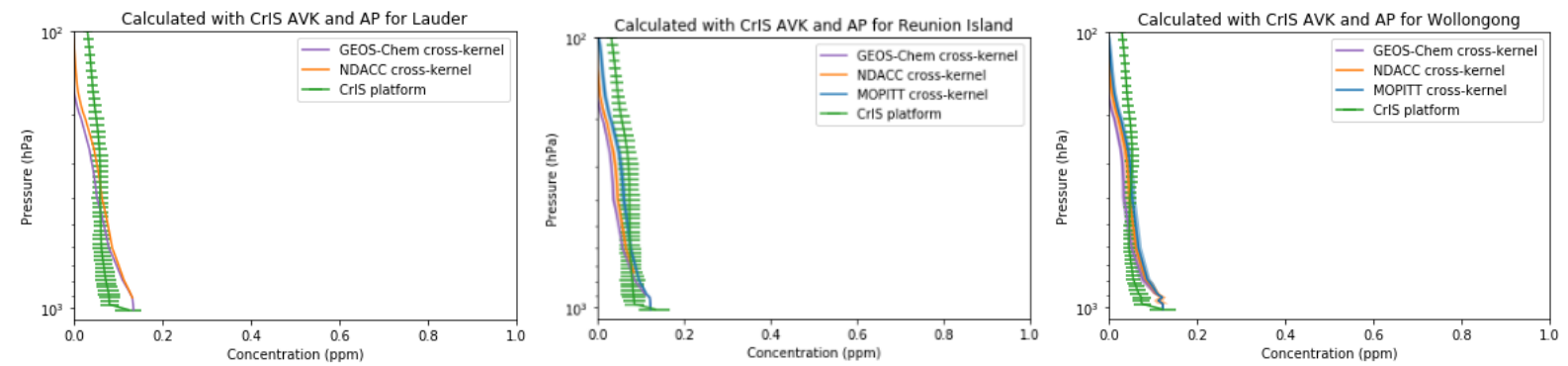

Figure 4.1.4.2: CO profile CrIS cross-kernel comparisons for Lauder on May $11^{\text {th }}$, Reunion Island on May $30^{\text {th }}$, and Wollongong on May $30^{\text {th }}$. The CrIS cross-kernel calculation weights the other observing platforms' upper atmosphere concentrations to zero.

There are multiple instances throughout Section 4.1 where CO comparisons between observing platforms show Altzomoni to be an outlier. This is likely due to the limited amount of data points for comparison, and therefore does not provide representative mean values. There are only 7 NDACC points over the four months, with only 2 points being comparable with MOPITT and 6 points being comparable with CrIS. The 2 MOPITT coinciding points may explain the abnormally high MOPITT cross-kernel total column percent differences for Altzomoni against NDACC, CrIS and GEOS-Chem (Figures 4.1.1.1 and 4.1.1.3).

Ny Alesund also has a limited amount of data points for comparison, with only 7 points between all instruments over the four months. Of which only one point is within August. This single point is why an increase in RMSE is observed in August between NDACC and CrIS, and NDACC and GEOS-Chem (Figures 4.1.2.4, B.2.1, and B.2.2). Closer inspection of the NDACC 
cross-kernel profile for this day also reveals a shape difference between these platforms above the boundary layer (Figure 4.1.4.3). Both NDACC and MOPITT observe a higher concentration within the free troposphere than the BL, whereas CrIS and GEOS-Chem do not. This is likely the result of using a 2019 observation for CrIS, which does not reflect the 2016 NDACC and MOPITT observations. Interestingly, the GEOS-Chem profile shape is similar to the CrIS observation. In this case, it is possible that the GEOS-Chem and CrIS represent a more typical observation for this time of year over Ny Alesund, and NDACC and MOPITT are observing an anomaly in 2016. For example, the anomaly in the mid troposphere could be the result of fire transport from another region. A similar anomaly is found to a lesser extent for Jungfraujoch and Zugspitze for the MOPITT cross-kernel comparison with GEOS-Chem. Where higher observed CO in the upper atmosphere is not represented in the GEOS-Chem model, causing a greater percent difference in August (Figure 4.1.1.3).
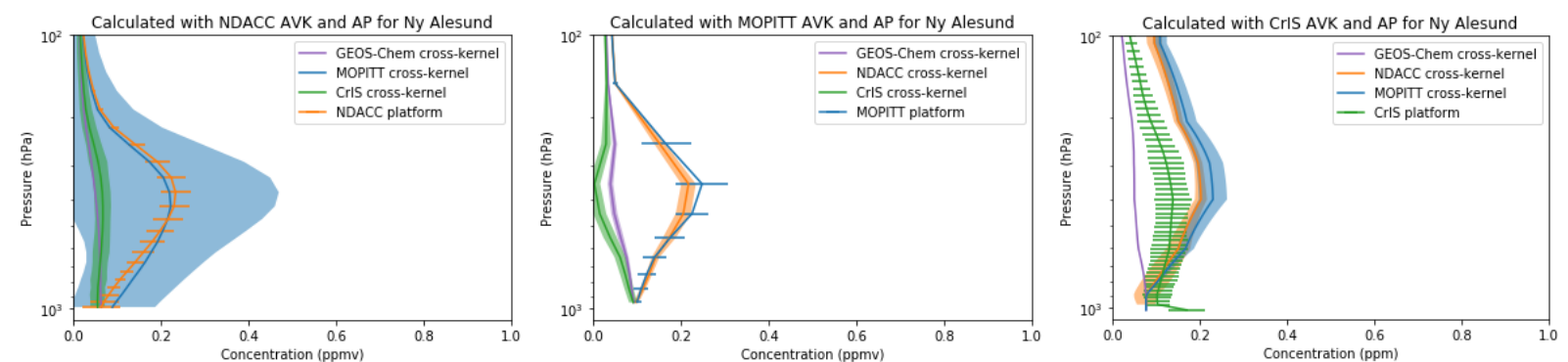

Figure 4.1.4.3: Profile cross-kernel comparisons between each observing platform over Ny Alesund on 2 August. Note that the CrIS observation is for the year 2019, and all other observations are for 2016. Across all cross-kernel comparisons, a characteristic bulge is seen for the NDACC and MOPITT observations, possibly indicating a 2016 anomaly event.

Like Ny Alesund, the Lauder profile cross-kernel comparisons show a large difference between 2016 observations and the 2019 CrIS observations. Except, the large difference is observed throughout all four months rather than one single date. Figure 4.1.4.4 below exhibits 
the typical characteristic Lauder CrIS profile shape which varies in magnitude, but the difference is always large. The shape tends to show maximum concentrations near the surface and $100 \mathrm{hPa}$, and a minimum concentration mid-troposphere. Breaking down the profile crosskernel calculation, this swerving shape only appears after the NDACC averaging kernel is applied. Looking at individual profiles without the cross-kernel calculations applied (Figure 4.1.4.5), the CrIS 2019 data points are observing a consistently different profile concentrations within the boundary layer than NDACC in 2016. This high surface concentration is then accentuated throughout the full profile once the NDACC averaging kernels are applied, leading to the high CrIS RMSE values in every profile section.
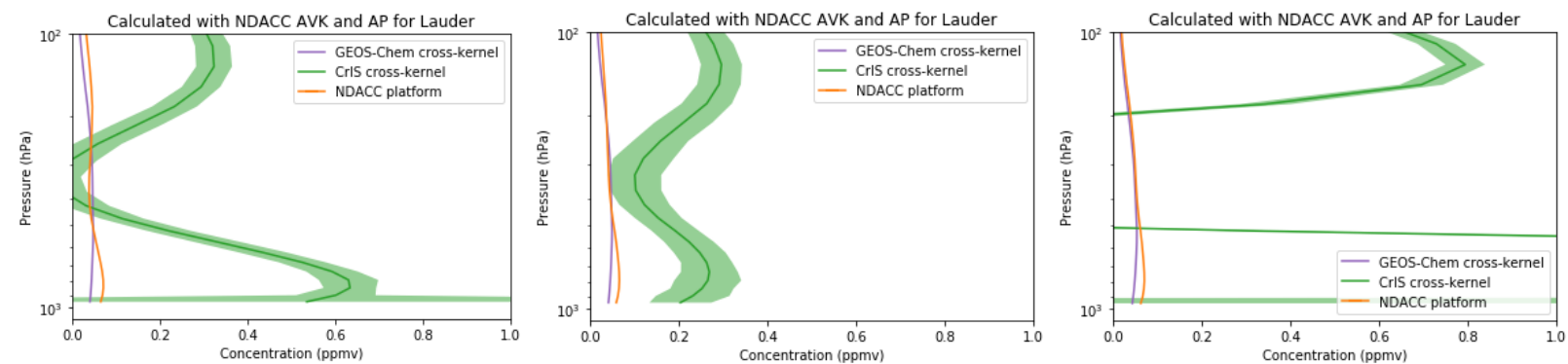

Figure 4.1.4.4: CO profile comparison with the NDACC cross-kernel calculation for Lauder on June $2^{\text {nd }}$, July $1^{\text {st }}$, and Aug $10^{\text {th }}$ (left to right).

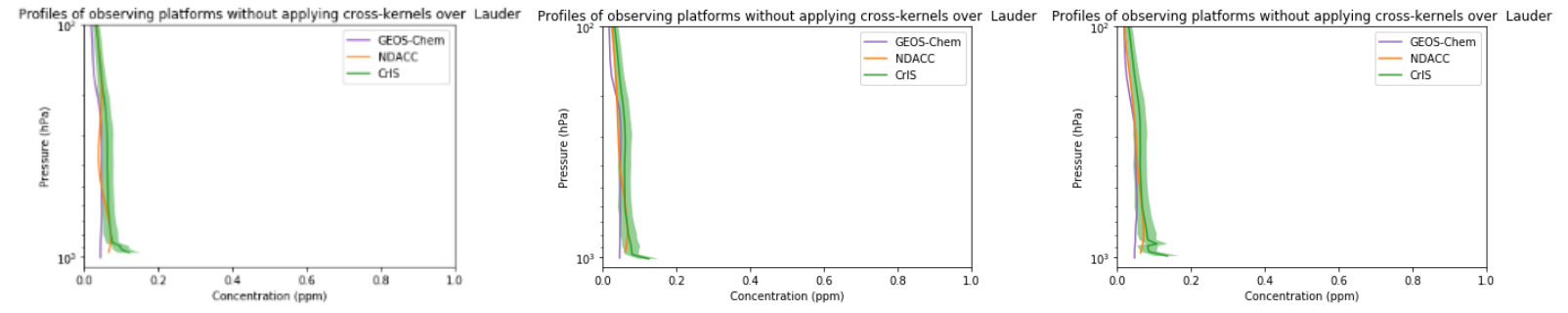

Figure 4.1.4.5: CO profile comparison with no cross-kernel calculations applied for Lauder on June $2^{\text {nd }}$, July $1^{\text {st }}$, and Aug $10^{\text {th }}$ (left to right). 
Aside from outlying comparisons and unexplained observations discussed above and in Section 4.1.5, MOPITT currently best represents CO profiles and total columns in this intercomparison study against the NDACC network. Analysis shows MOPITT has the strongest agreement over all analysed troposphere sections both in MOPITT cross-kernel and NDACC cross-kernel calculation analyses. MOPITT CO is a mature data product and has matured over the years through many validation studies, leading to its high agreement. Therefore, it is a suitable candidate to assimilation into the GEOS-Chem model to infer global $\mathrm{OH}$. Recent studies have already started the investigation into assimilating MOPITT CO observations into global models as an alternative tracer to determine $\mathrm{OH}$ temporal and spatial trends (Gaubert et al., 2017; Jones, 2019; X. Zhang et al., 2019).

CrIS also provides a strong CO profile agreement with NDACC, but only above the boundary layer due to less sensitivity at altitudes below $700 \mathrm{hPa}$ (Smith et al., 2020b). Therefore, the CrIS total columns best match NDACC stations located at high elevations. As a newer product with less validation studies, CLIMCAPS appears to have stronger weighting to the a priori, especially within the boundary layer. Monthly trends show the expected TIR instrument characteristic increase in profile DOFS and accuracy by August, due to increasing thermal contrast from insolation (Deeter et al., 2015). However, without using CO profiles from the same year for intercomparison the accuracy of the CLIMCAPS dataset is not confirmed. Assimilation of CrIS observations into the GEOS-Chem model may not be able to adjust surface $\mathrm{CO}$ emission and boundary layer conditions as well as MOPITT. Although, its significantly greater spatial and temporal resolution may be capable of providing superior information about $\mathrm{OH}$ distribution above the boundary layer. 
TROPOMI also provides a promising CO total column agreement with MOPITT. However, without proper cross-kernel analysis its evaluation against MOPITT and NDACC hold limited value. Without more information on the TROPOMI CO profiles or averaging kernels, the particularly high DOFS cannot be explained either. Assuming that the strong agreement with MOPITT and high DOFS are valid, assimilation into GEOS-Chem could provide a better constraint on $\mathrm{CO}$ emissions and boundary layer conditions. Its greater spatial and temporal resolution may therefore be capable of providing superior information on $\mathrm{OH}$ distribution within the boundary layer. This being said, the future Joint CrIS-TROPOMI data product has the potential to provide superior information on $\mathrm{OH}$ distribution than MOPITT throughout all layers of the troposphere.

\subsubsection{Sources of Error and Bias}

Limitations, sources of error, and bias must be taken into consideration when reviewing observations in the sections above. The largest limitations of this study are the temporal and spatial ranges used. The temporal range from May to August 2016 was chosen because all NDACC stations had complete 2016 CO datasets ready for download at the beginning of this study in Fall 2018. MOPITT and GOES-Chem 2016 data is used for comparison, but TROPOMI and CrIS 2019 datasets are used instead of 2016. TROPOMI 2019 data was chosen as the earliest TROPOMI data products available start midsummer in July 2018 (Copernicus Sentinel5P (processed by ESA), 2018). Although CrIS's CLIMCAPS data product is available for all months in 2016, investigation revealed that observations within these datasets only contained a priori information (N. Smith, personal communication, September 15, 2020). Therefore, 2019 CLIMCAPS data was instead to match TROPOMI. Using observations from difference years 
impact each daily comparison between CrIS and TROPOMI against the other observing profiles. Since they are not observations from the same year, profile RMSE and total column percent difference values are expected to be larger than if the observations were made within the same year. Since these observations are still over the same months, averaged observations hold more value than specific individual profile and column observations.

The four-month time span of this study limits the number of coinciding CO data to analyse between observing platforms. The number of daily NDACC observations available between May and August 2016 varies by station, with generally fewer observations in the Southern Hemisphere due to less daylight. Lack of daylight during these months also eliminates any comparisons with the Arrival Heights NDACC station in Antarctica. As MOPITT does not have daily global coverage, the May to August timeframe also limits the number of coinciding MOPITT observations over NDACC stations. Additionally, not all coinciding times contained valid data. No comparisons were made over Paramaribo station due to limited NDACC observations and no coinciding MOPITT overpass times. Due to these limitations, compensations were made. Satellite observations were accepted within a $200 \mathrm{~km}$ radius of each NDACC station within \pm 4.0 hours of the NDACC observations. This allowed for more satellite observations to be cross compared with the NDACC stations.

Averaging observations over larger areas introduces additional bias. An averaged observation over a larger surrounding area may not be representative of the selective NDACC observation. For example, an NDACC station may be in an urban centre with higher CO concentrations but the surrounding area is rural with low CO concentrations, which would decrease the averaged satellite observation. Alternatively, there may not be a satellite 
observation directly over the NDACC station. For example, if satellite quality indicators eliminated observations over the NDACC station, averaged observations may be of an area 100 to $200 \mathrm{~km}$ away from the station.

Figures 4.1.5.1 through 4.1.5.7 show timeseries and boxplots of each instrument's averaged daily total error. As mentioned in Section 3.2, profile and total column total error is calculated as the variability between the averaged points plus the averaged instrument error. Figure 4.1.5.1 is a timeseries plot of the daily average total column error for all instruments, and Figure 4.1.5.2 shows the error distribution between stations in a boxplot. NDACC has the largest average variability over time and between stations as it has a selective single column view. Note that Lauder error is not shown in Figure 4.1.5.2 as error values were not provided in the NDACC Lauder dataset. CrIS and MOPITT have comparable variability, and TROPOMI has the smallest variability over time and between stations. The significantly smaller TROPOMI error and variability may be influenced by its significantly larger DOFS, limiting the window of error.

This Figure 4.1.5.1 also reveals negative slope over time for the CrIS and MOPITT instruments. This is likely reflective of increasing insolation and thermal contrast by August, which in turn increases the DOFS of TIR observing instruments (Deeter et al., 2015) and therefore minimises error. TROPOMI has a slight positive slope over time. This is likely related to the decrease in CO loading in the Northern Hemisphere by August, which in turn decreases the DOFS of the NIR observing instrument (Deeter et al., 2015) and therefore increases error.

NDACC total column error also has a positive slope in Figure 4.1.5.1. Since NDACC station data are single columns rather than averaged over an area, its error is sensitive to each 
individual NDACC station location. The positive slope may be indicative of a trend, but this observation is limited without analysing averaged error over multiple seasons or years. In Figure 4.1.5.2, large differences in error ranges can be seen between NDACC stations. The majority of NDACC station error ranges are below $5 \times 10^{17}$ molecules $/ \mathrm{cm}^{2}$, and six stations have significantly larger error: Altzomoni, Izana, Jungfraujoch, Kiruna, Reunion Island, and Zugspitze.

These are the same six high elevation stations (except for Kiruna) mentioned in Section 4.1.1, that have a small total column percent difference with CrIS observations. Varying weather conditions and minimal CO loading at their higher elevations may be impacting CO total column error. It may also be possible that these larger total column errors more reflective of upper atmosphere errors, which would explain why these stations' errors stand out in Figure 4.1.5.2 but not in Figure 4.1.5.5 profile errors below $100 \mathrm{hPa}$. However, it is unknown why Kiruna is again grouped with these five high elevation stations, like the small total column percent differences with CrIS found in Section 4.1.1. It is also unknown why the high elevation station Mauna Loa is not included in this grouping. This would require further investigation into NDACC station individual retrieval methods.

Figures 4.1.5.3 and 4.1.5.4 are timeseries plots of the profile error for the $100 \mathrm{hPa}$ to $\mathrm{BL}$, and BL sections respectively. The full profile and below $100 \mathrm{hPa}$ profile sections are provided in Appendix B.2, as they do not reveal much additional information for this analysis. All three instruments in Figures 4.1.5.3 and 4.1.5.4, show negative slopes indicating less error by August. Again, this matches the increasing DOFS due to insolation and thermal contrast for the TIR observing instruments MOPITT and CrIS, decreasing error. NDACC's decreasing profile error 
over these sections may also be attributed to insolation, allowing for more daylight measurements with clear skies.

Figures 4.1.5.5 through 4.1.5.7 are boxplots showing the distribution of daily average profile errors for different profile sections. CrIS consistently has the largest average profile error for each section, which is likely attributed to CLIMCAPS being the newest and therefore less mature product compared to MOPITT. CrIS's largest errors occur within the boundary layer, again reflective of CrIS's low sensitivity to altitudes below $700 \mathrm{hPa}$ (Smith et al., 2020b). NDACC consistently has the smallest average profile error for each section, except within the boundary layer (Figure 4.1.5.7) where error average closer matches to MOPITT. However, NDACC does not have observations within the boundary layer for the high elevation stations, whereas MOPITT does due to the $200 \mathrm{~km}$ radius. MOPITT error ranges are similar between each profile section except for Rikubetsu station, where the error range is significantly larger (Figure 4.1.5.7). Throughout this study, Rikubetsu observations and cross-comparisons do not stand out from usual trends. The larger error range may be attributed to local weather, as Rikubetsu 


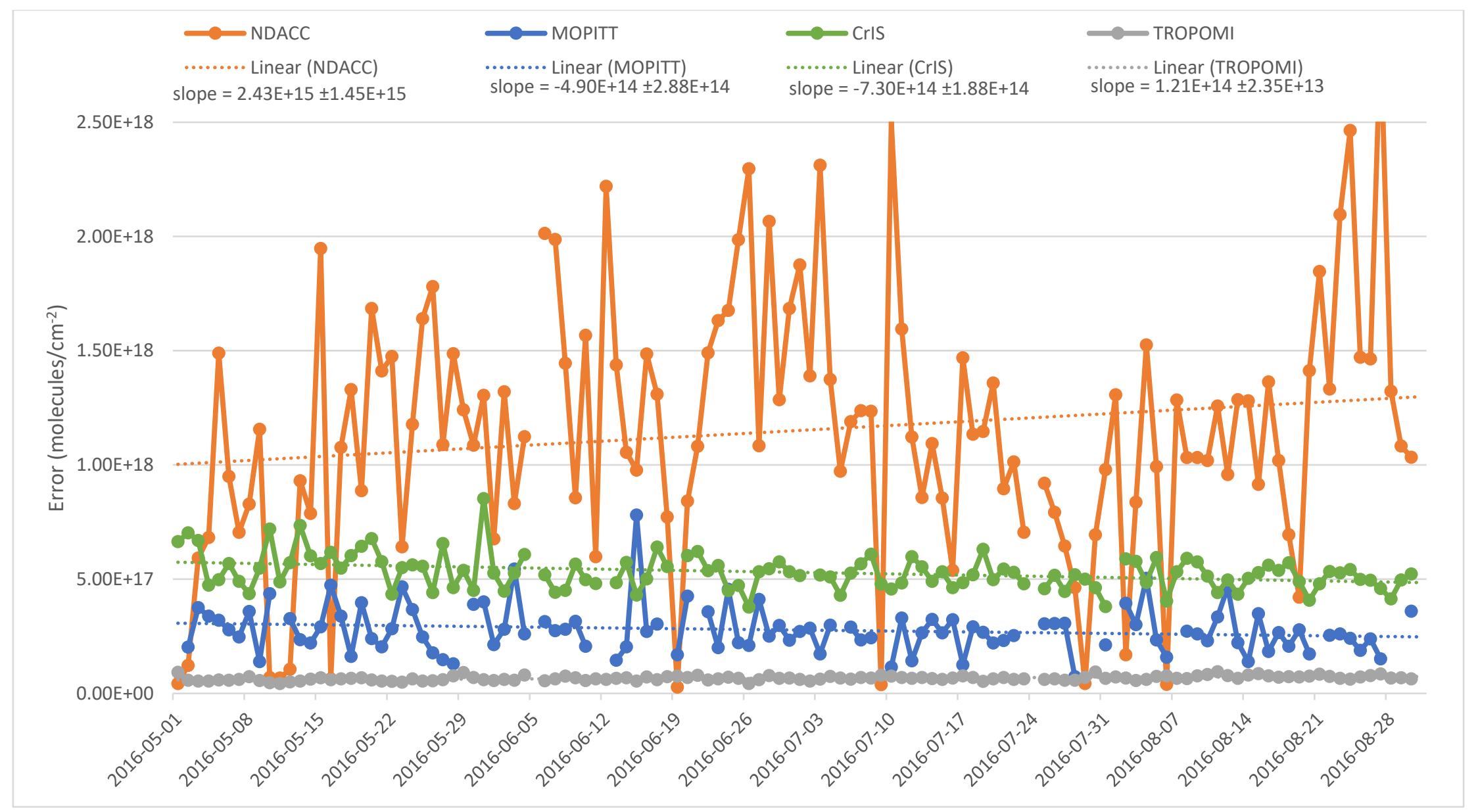

Figure 4.1.5.1: Daily average CO total column error average for each observing platform for May through August 2016. The total column error is the provided dataset error values plus the variability of the averaged points. 


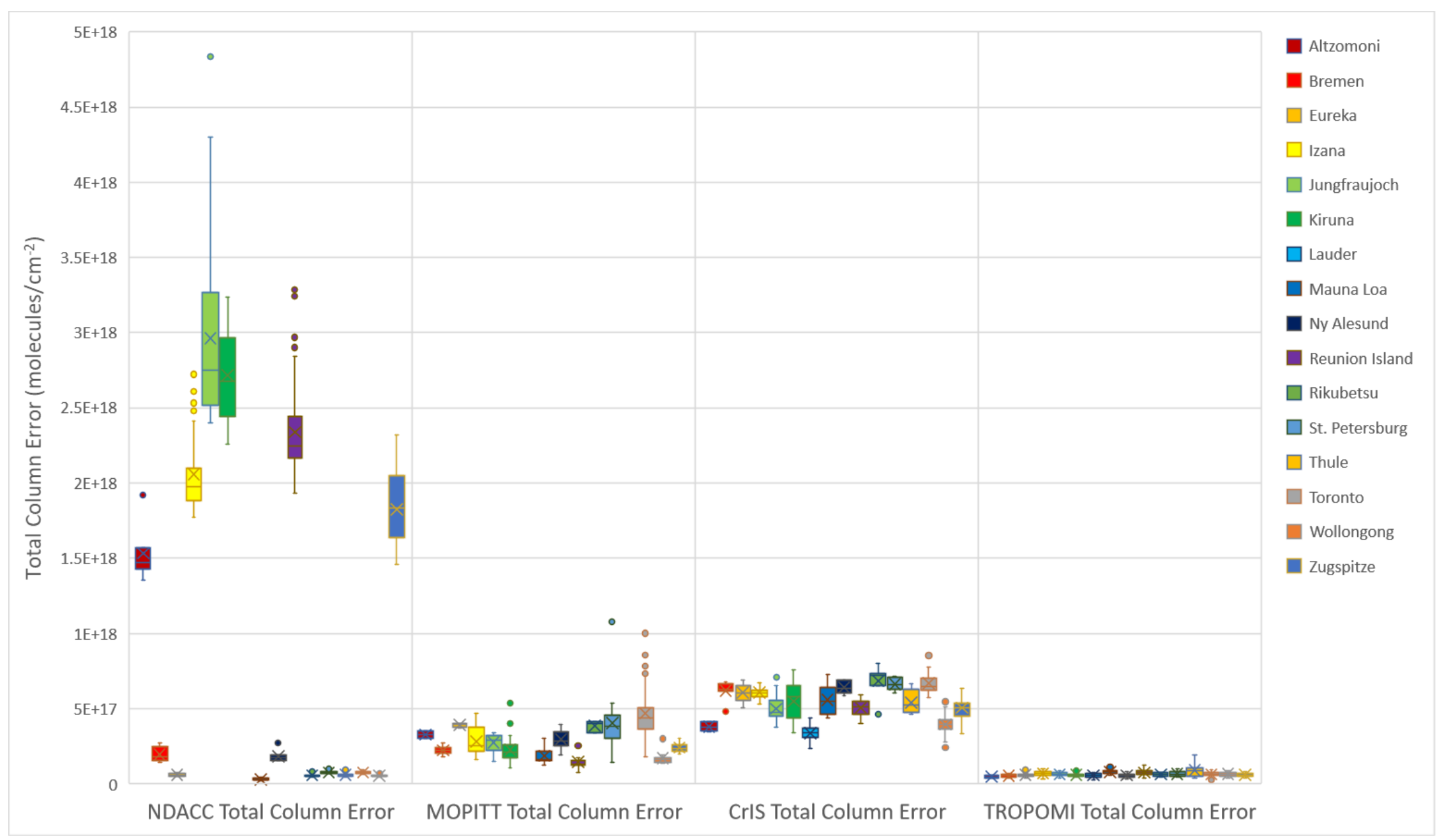

Figure 4.1.5.2: Distribution of daily average CO total column error per station for May through August 2016. The total column error is the provided dataset error values plus the variability of the averaged points. 


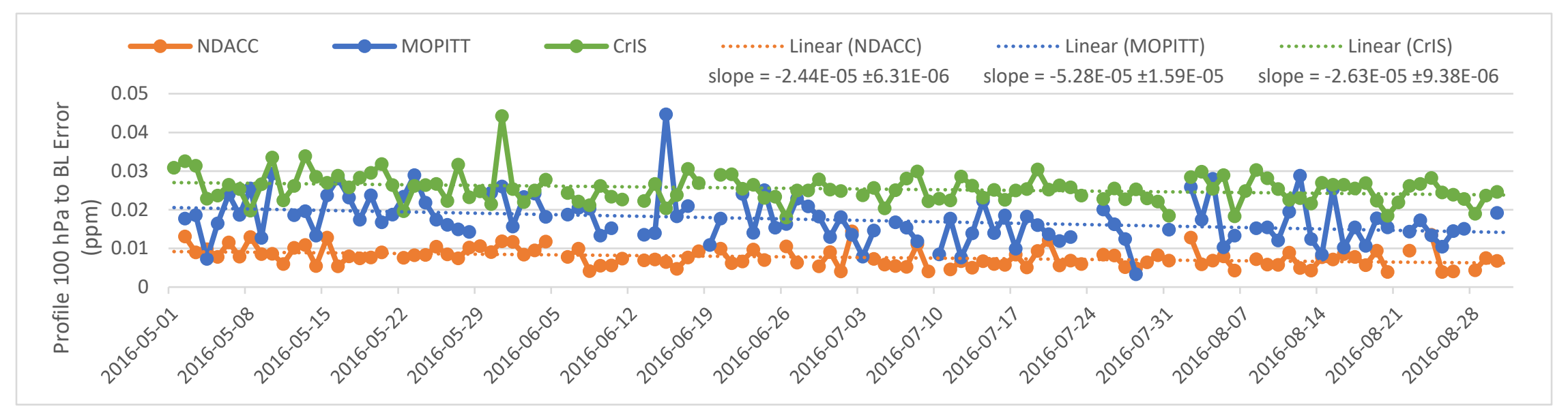

Figure 4.1.5.3: Daily average CO profile error from $100 \mathrm{hPa}$ to BL for each observing platform for May through August 2016. The profile errors are the provided dataset error values plus the variability of the averaged points.

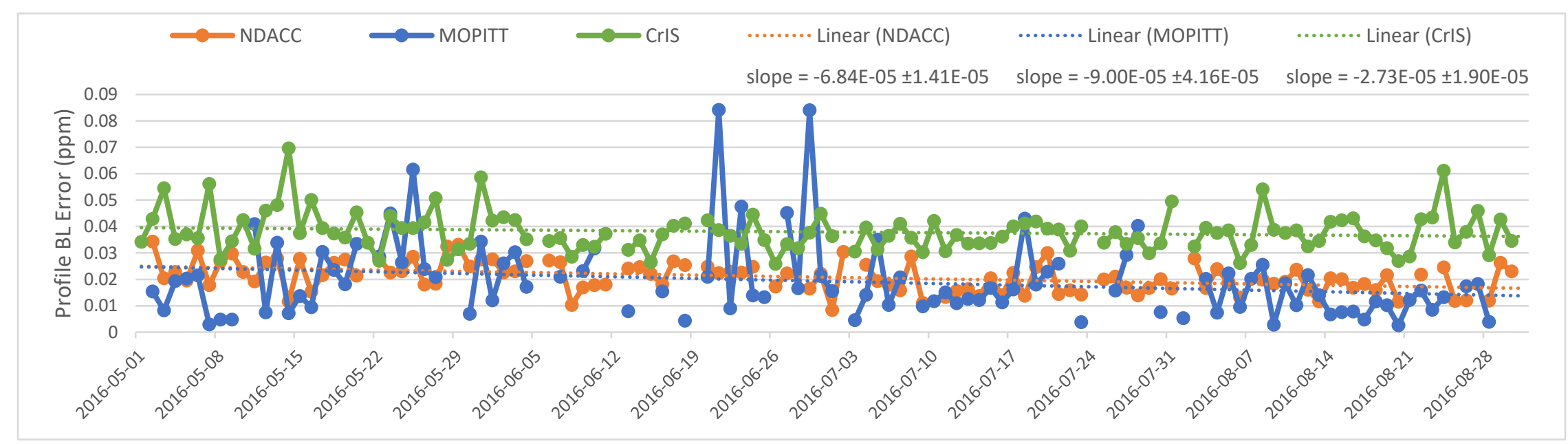

Figure 4.1.5.4: Daily average CO profile error within the BL for each observing platform for May through August 2016. The profile errors are the provided dataset error values plus the variability of the averaged points. 


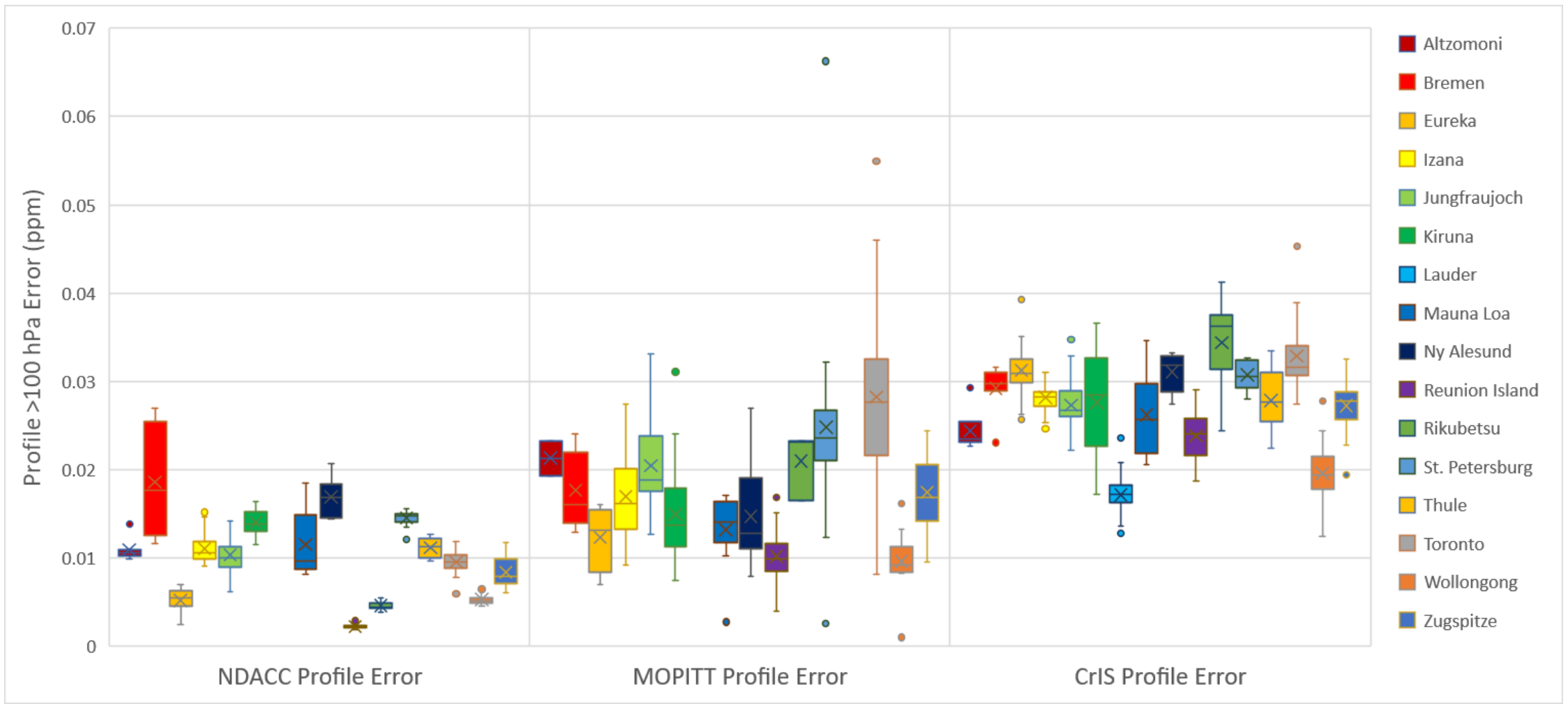

Figure 4.1.5.5: Distribution of daily average CO profile error per station from the surface to 100 hPa for May through August 2016. The profile errors are the provided dataset error values plus the variability of the averaged points. 


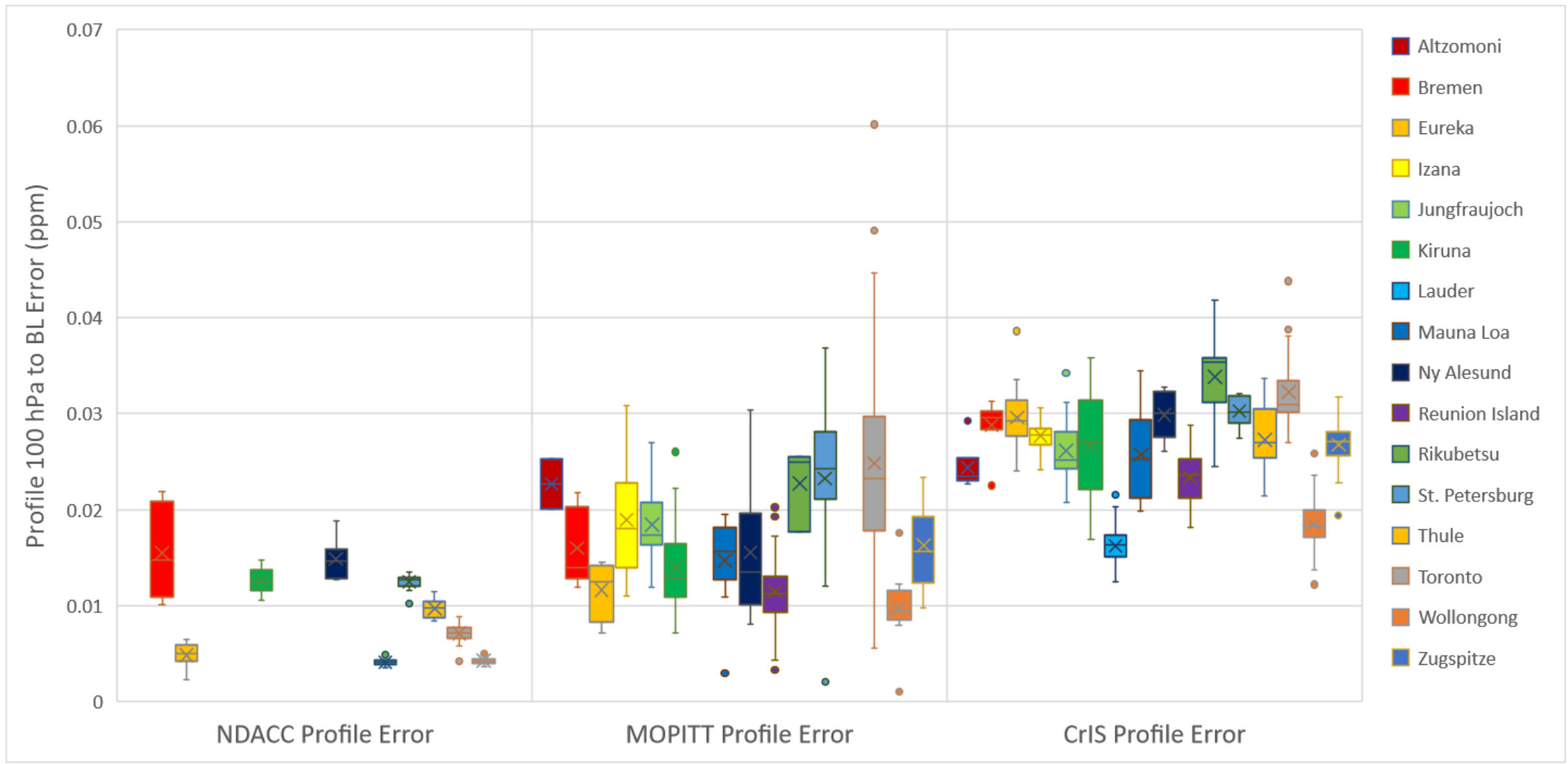

Figure 4.1.5.6: Distribution of daily average CO profile error per station from $100 \mathrm{hPa}$ to BL for May through August 2016. The profile errors are the provided dataset error values plus the variability of the averaged points. 


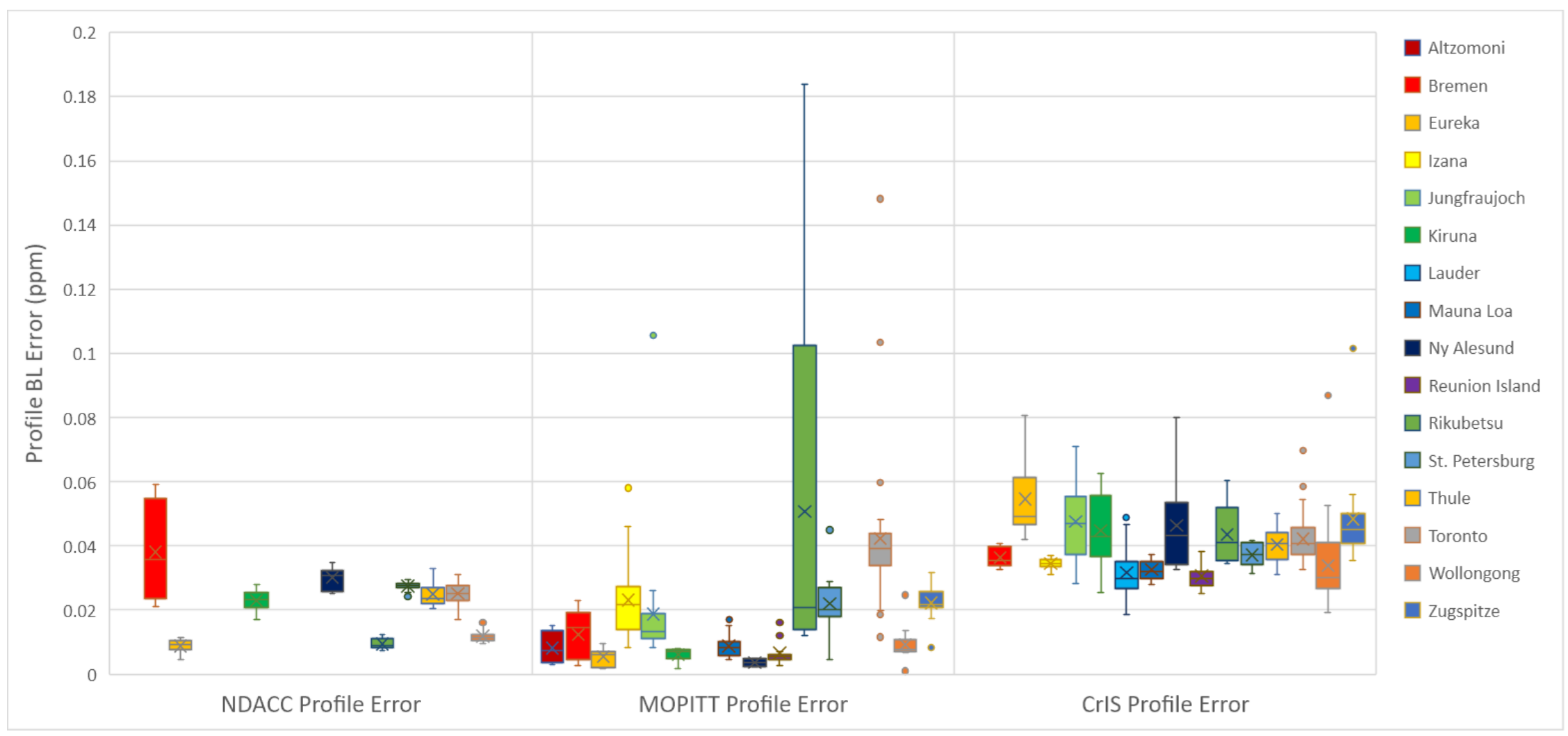

Figure 4.1.5.7: Distribution of daily average CO profile error per station within the BL for May through August 2016. The profile errors are the provided dataset error values plus the variability of the averaged points. 
is in between mountain ranges and may be subject to inversions or trapped particulates (low level haze or clouds).

In addition to temporal and spatial limitations introducing error and bias, there are also the dataset limitations. As mentioned in Section 3.2, not all observing platform datasets contain the same units or parameters required for direct comparisons. As seen in Table 3.2.1.1, CrIS total column cross-kernel comparisons and TROPOMI total column and profile cross-kernel comparisons cannot be made. Their datasets to not contain products required for the crosskernel calculations. This limits the study's analysis as true cross-kernel comparisons cannot be made.

Not all observing platform data sets have the same units either. Since CrIS's profile is provided in partial column units of molecules $/ \mathrm{m}^{2}$, conversion calculations need to be made before applying profile cross-kernel calculations. For these conversions, air density and layer altitude boundaries are required. However, not all observing profile data products have these parameters (Table 3.2.2.1). Instead, air density is calculated using temperature, altitude, and pressures. For MOPITT, these parameters are taken from the CAM-Chem data product. These calculated conversions assume uniform air density between levels, introducing numerical calculation error and bias from the use of different datasets to calculate air density.

Since each observing platform provides profile CO concentrations at different pressure levels and layers, interpolations are applied to each profile to match the comparing platform's for the cross-kernel calculation (see Section 3.2). Interpolation of air density profiles are also calculated for conversions. The selected Python package scipy.interpolate.UniverariateSpline 
appears to interpolate between profiles well, maintaining the profile shape including when there are sharp CO transitions between altitudes. However, this package appears to struggle with specific extrapolations for certain profiles where the surface value is severely overestimated, or the TOA value is underestimated. These instances have been identified and minimised by manually adjusting the smoothing factor of the spline function (see Section 3.2.2).

With most NDACC 2019 data available today, soon a 2019 direct temporal comparison will be possible. This would allow for true representative cross-kernel comparisons between all observing platforms. For a continuation of this study with 2019 data, it is recommended that an entire year be analysed to allow for more potential MOPITT and NDACC coinciding times for comparison. More comparison incidences should allow for minimising the acceptable satellite radius to $100 \mathrm{~km}$ from $200 \mathrm{~km}$, reducing spatial errors. Should future TROPOMI and CLIMCAPS product versions provide the required information, TROPOMI and CrIS total column crosskernel comparisons should also be conducted. In addition, it would be beneficial to also crosscompare the Joint CrIS-TROPOMI data product once available. This would provide insight into how each instrument's retrieval performs individually compared to when joined together. 


\subsection{Part 2: Sensitivities}

Part 2 contains observations and evaluations of observing platform sensitivities within the GEOS-Chem adjoint model after one iteration. Section 4.2.1 presents modeled global CO distribution, and global CO emission sensitivities. Differences in global CO distribution were minimal but reveal some zonal differences, and potential error caused by the modeled variable tropopause. CO emission sensitivity analysis demonstrates MOPITT's significantly greater assimilation affect due to global coverage on global $\mathrm{CO}$ (and therefore $\mathrm{OH}$ ) than the NDACC network. Section 4.2.2 presents modeled global $\left[\mathrm{CH}_{4}+\mathrm{OH}\right]$ reaction rate sensitivities. MOPITT again demonstrates that its greater global coverage heavily influences model $\mathrm{OH}$ chemistry. The observing platforms evaluated are limited to the NDACC and MOPITT instruments. Evaluations of CrIS and TROPOMI model assimilations are not included due to time constraints but are recommended for future studies.

\subsubsection{CO Sensitivities}

Before comparing sensitivities to CO emissions, the 2016 GEOS-Chem base model CO concentrations are compared against the single iteration MOPITT assimilation. The global CO distribution between them is indistinguishable from each other with minor differences. The GEOS-Chem base model shows more CO in the Northern Hemisphere in May, with surface concentrations highest between $40^{\circ}$ and $60^{\circ}$ latitude and a relatively high $\mathrm{CO}$ amounts at the North Pole just above the boundary layer (Figure 4.2.1.1). Global CO appears to be lower in June and July, then high in August between $0^{\circ}$ and $-10^{\circ}$ latitude due to events over Africa and South America. Modeled CO distribution between $-40^{\circ}$ and $-90^{\circ}$ appears stable throughout all four months, with minimal CO intrusion in the mid-troposphere from higher latitudes in July 
and August. The MOPITT assimilation model results present these same characteristics and are shown in Appendix B.3.

Although the $\mathrm{CO}$ distributions are nearly identical after one iteration, there are minor spatial differences between them. Figure 4.2.1.2 shows the MOPITT integrated CO distribution minus the GEOS-Chem base distribution. Blue represents where the GEOS-Chem base and MOPITT integrated models output the same CO concentrations. Purple represents where the GEOS-Chem base model outputs higher concentrations, and yellow represents where the MOPITT integrated model outputs higher concentrations. In May, most of the plot area is blue showing agreement between the two model runs. However, there are scattered minor positive and negative differences $\left( \pm 2.0 \times 10^{-9} \mathrm{ppm}\right)$ spaced out across the vertical and horizontal planes within the Southern Hemisphere. The random appearance in differences likely reflect numerical noise between the model runs, which is expected. Interestingly, this pattern only appears within the Southern Hemisphere possibly indicating a model bias between the Northern and Southern Hemisphere. Perhaps the Northern Hemisphere model portion is more mature and backed up by validation studies, whereas the Southern Hemisphere may be as a less mature product with more numerical estimations. In May, there is also a slight purple band spanning the Pacific just south of the Equator, indicating greater GEOS-Chem concentrations. This is possibly a slight model overestimation of natural emissions (Dalsøren et al., 2016). 
i) May 2016 GEOS-Chem CO concentrations

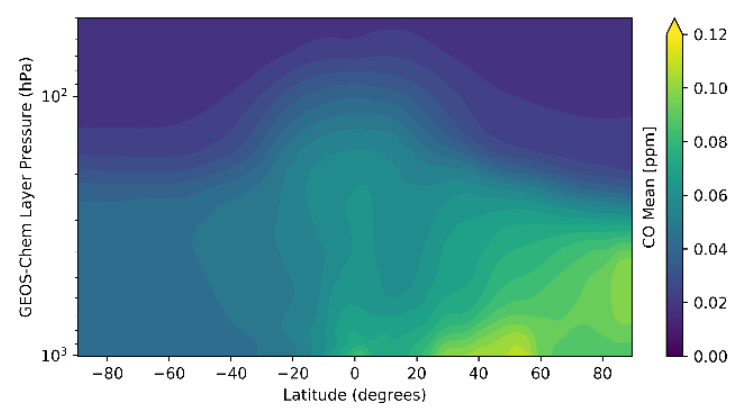

ii) June 2016 GEOS-Chem CO concentrations

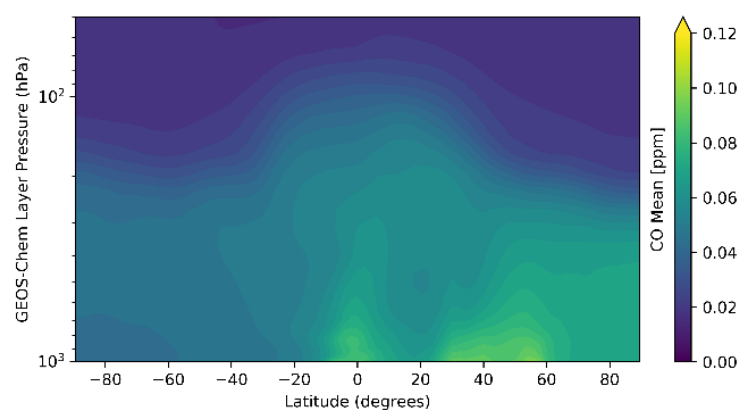

iii) July 2016 GEOS-Chem CO concentrations

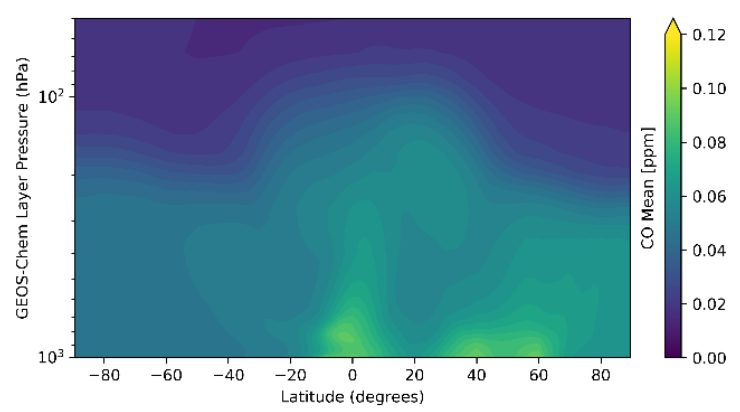

iv) August 2016 GEOS-Chem CO concentrations

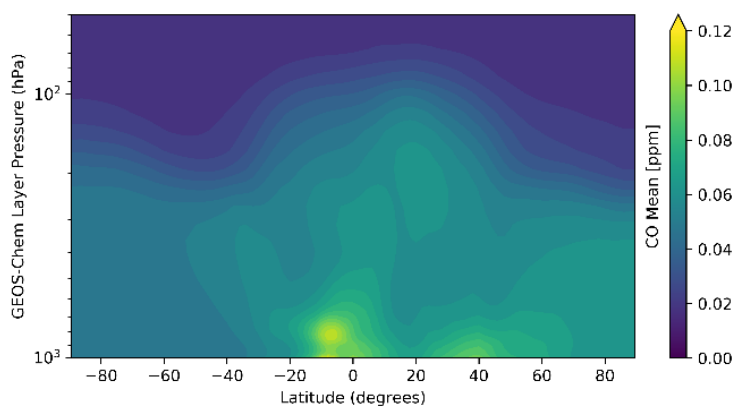

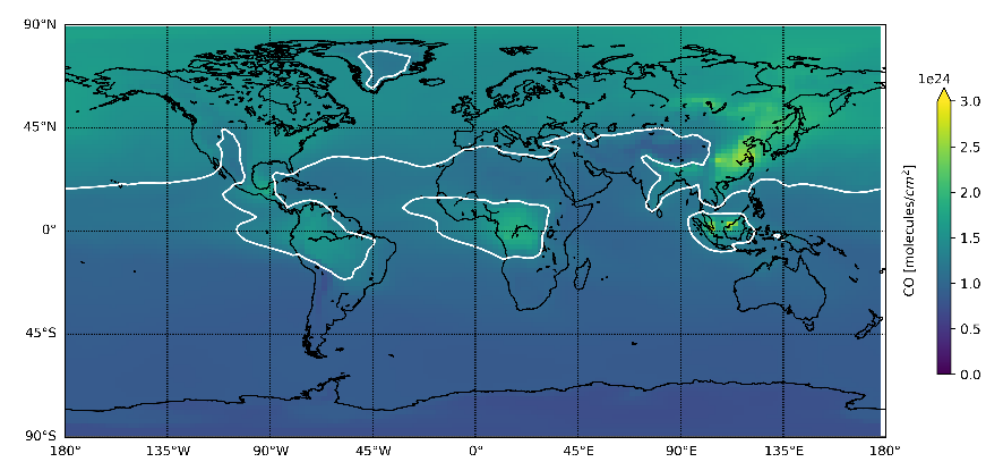
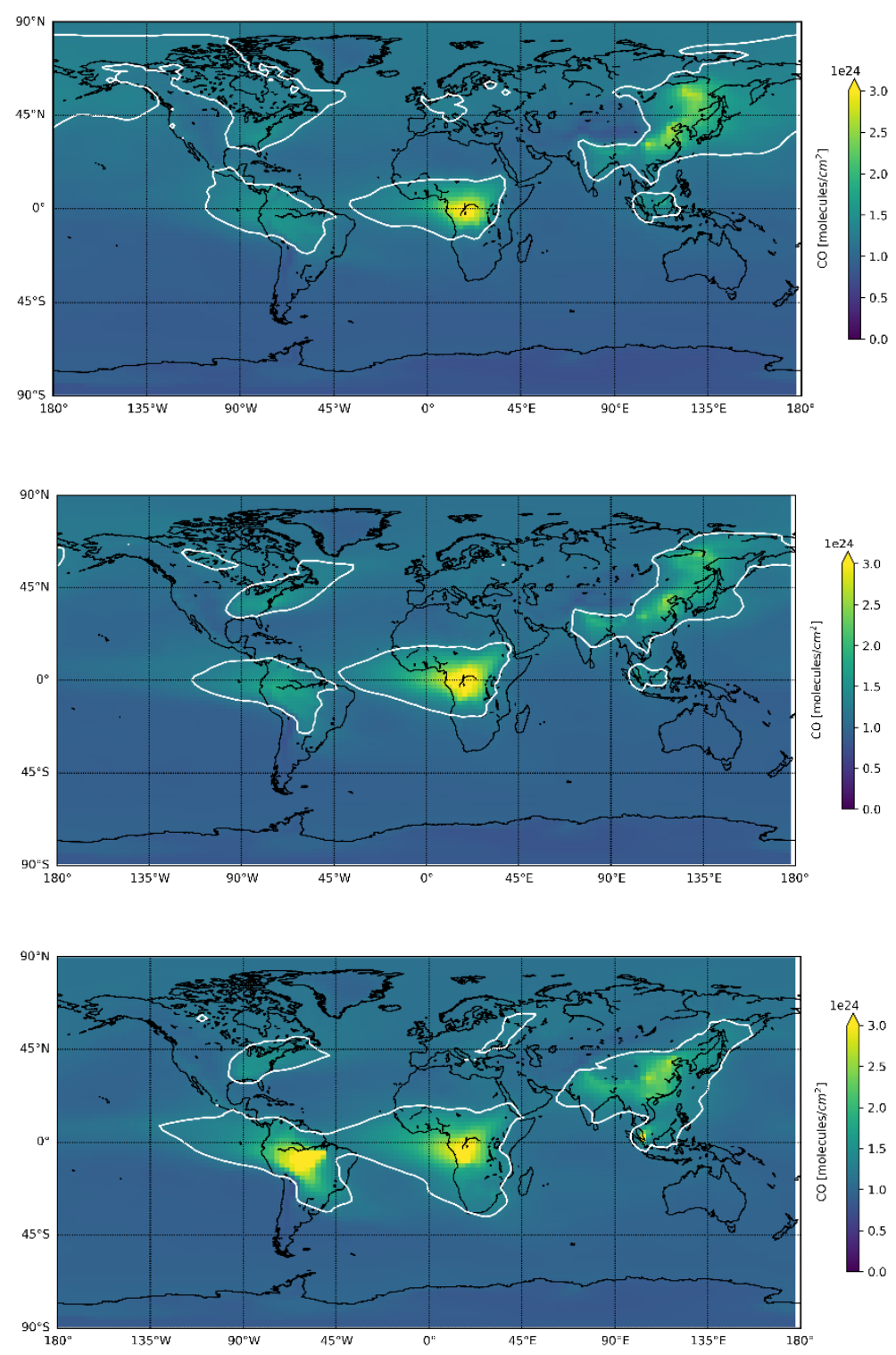

Figure 4.2.1.1: GEOS-Chem base modeled mean global CO distribution for each month, May through August 2016. Figures on the left represent the $\mathrm{CO}$ zonal mean ppm across the globe. Figures on the right represent to total column CO concentration in molecules $/ \mathrm{cm}^{2}$. The white contour line represents the MOPITT integrated model global CO concentration's $80^{\text {th }}$ percentile. 
In June, again, most of the plot area is blue showing agreement. The minor positive and negative differences in June lies within the upper troposphere over Africa and Asia between $10^{\circ}$ and $50^{\circ}$ latitude. Differences between the modeled CO distributions become larger in July and August, separating into three different bands. The first upper troposphere band of positive and negative differences observed in June, appears larger in July and August. It widens vertically reaching into the stratosphere and penetrates deeper into the troposphere, while spreading horizontally across the globe between $-40^{\circ}$ and $60^{\circ}$ latitude. The second band appears in July and August as a strong positive difference within the upper troposphere between $60^{\circ}$ and $90^{\circ}$ latitude. The third band is a positive difference between the surface and mid-troposphere, mostly within the Southern Hemisphere. In July, there is only a slight positive difference, but in August it grows stronger between $-50^{\circ}$ and $10^{\circ}$ latitude. Although these CO differences are small and indistinguishable between the GEOS-Chem base and MOPITT assimilation, it does reveal spatial and temporal disagreements. 
i) May 2016 MOPITT - GEOS-Chem CO difference

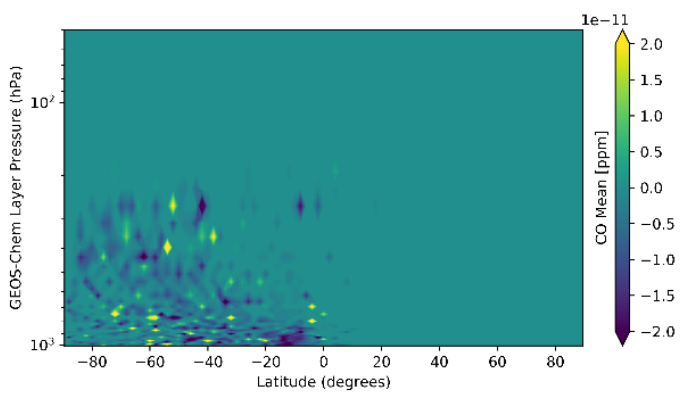

ii) June 2016 MOPITT - GEOS-Chem CO difference

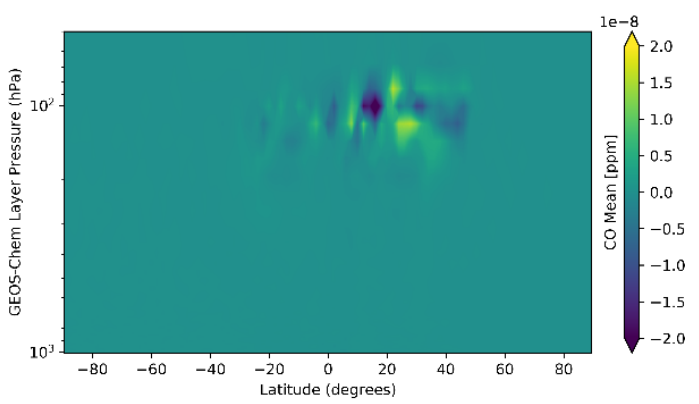

iii) July 2016 MOPITT - GEOS-Chem CO difference

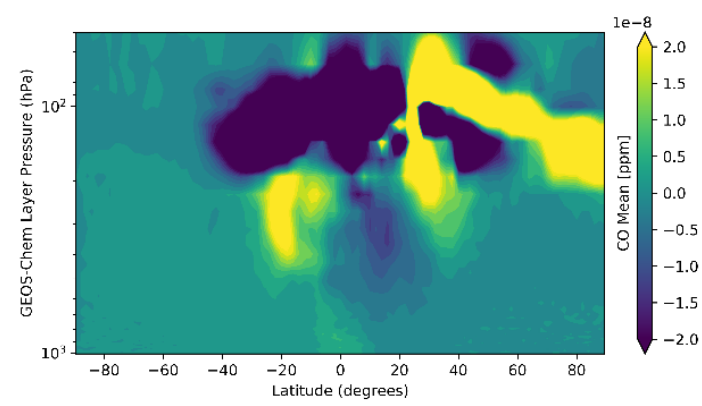

iv) August 2016 MOPITT - GEOS-Chem CO difference

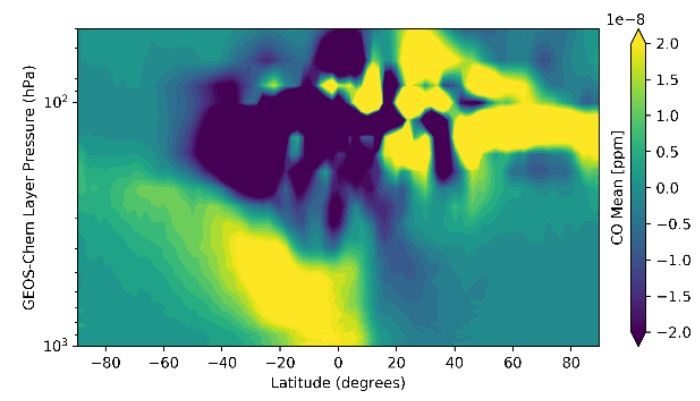

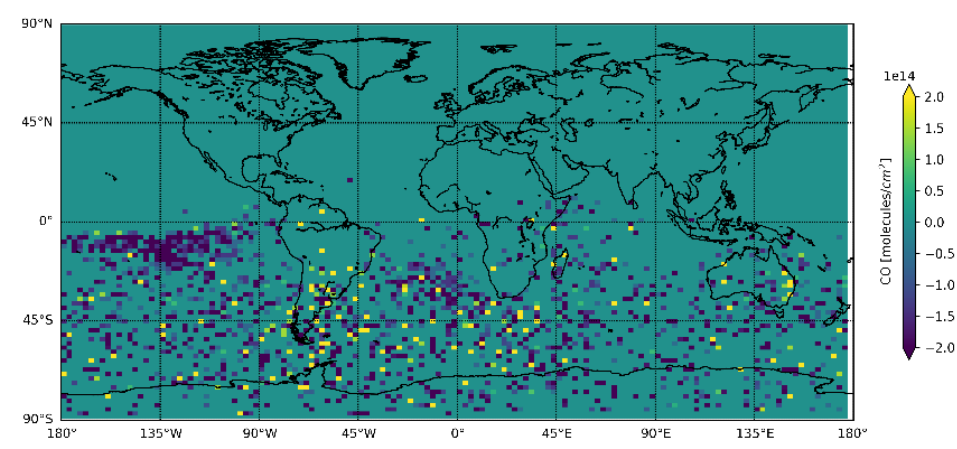
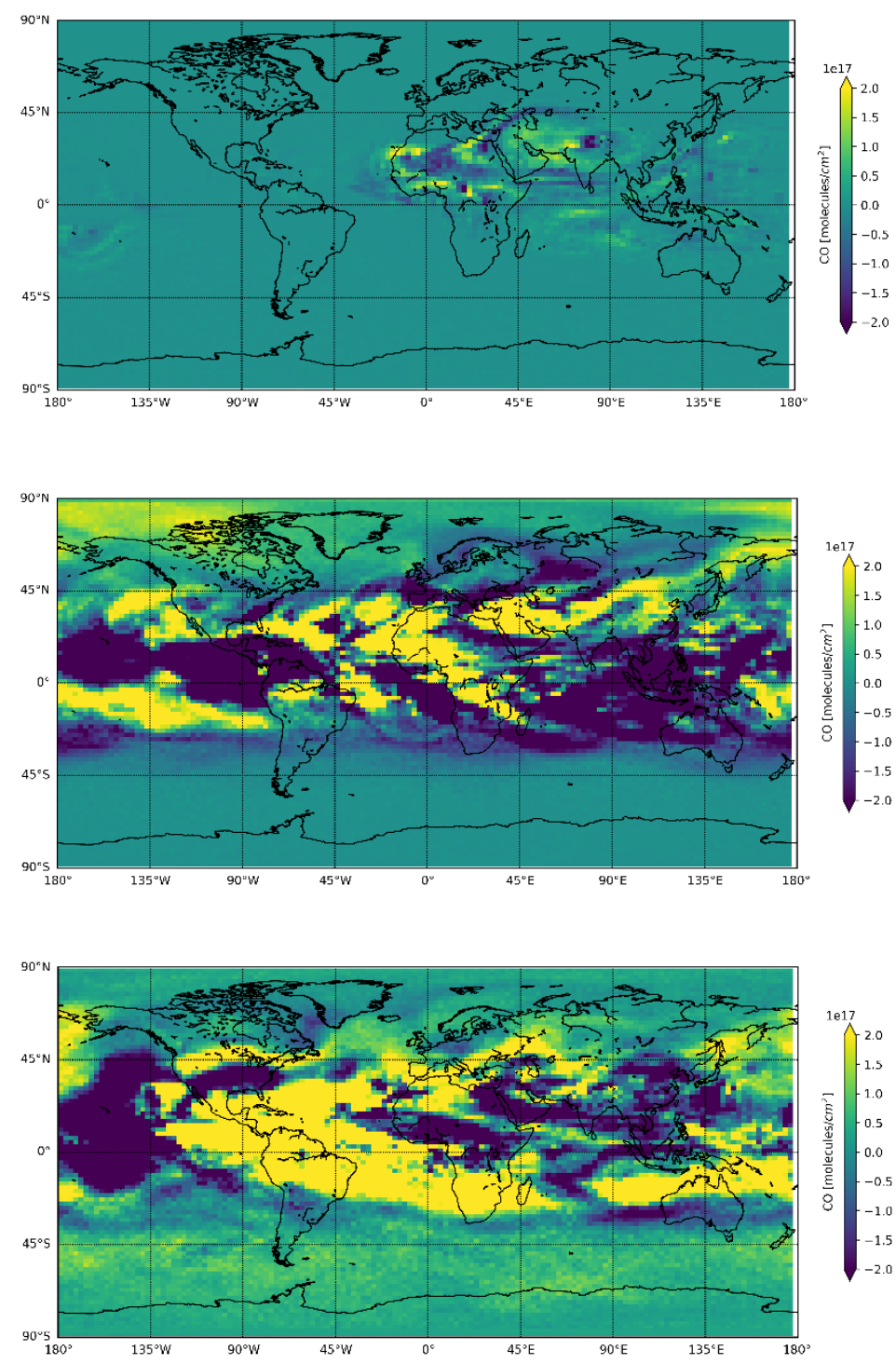

Figure 4.2.1.2: MOPITT minus the GEOS-Chem base mean global CO distribution and the for each month, May through August 2016. Figures on the left represent the CO zonal mean ppm difference across the globe. Figures on the right represent to total column CO concentration difference in molecules $/ \mathrm{cm}^{2}$. Note that the MOPITT CO distribution used is the model output after one adjoint iteration. 
Figures 4.2.1.3 to 4.2.1.5 and Figures 4.2.1.6 to 4.2.1.8 show the NDACC and MOPITT sensitivity to anthropogenic, biofuel, and biomass burning CO emissions within the GEOS-Chem model, respectively. Although their unitless sensitivity values are small (compared to the $\left[\mathrm{CH}_{4}+\right.$ $\mathrm{OH}$ ] reaction in Section 4.2.2), their spatial distributions are vastly different. Within these figures, red represents positive sensitivity and blue represents negative sensitivity. As stated in Section 3.3, positive sensitivity represents a model response where the cost function is increasing due to increasing changes in $\mathrm{CO}$ emissions. Negative sensitivity represents a model response where cost function is decreasing due to increasing changes in CO emissions.

The NDACC adjoint CO emission sensitivity is positive across all figures (Figures 4.2.1.3 to 4.2.1.5). They are also largely restricted by the locations of the individual NDACC stations. Sensitivity tends to be strongest within the Northern Hemisphere, specifically within Europe as this is where most NDACC stations are located. It is also observed that polar stations (Arrival Heights, Eureka, Thule and Ny Alesund) do not show sensitivity to any CO emissions, likely due to a lack of the specified emissions within these regions. Of all the NDACC Figures, the largest CO emission sensitivity is anthropogenic (Figure 4.2.1.3). May through August, the largest sensitivities are over eastern North America, Europe, Eastern Asia, and Eastern Australia. The sensitivity distribution does not vary much month to month, apart from sensitivity lost over Altzomoni in July. 
i) May 2016 NDACC Anthropogenic CO sensitivity

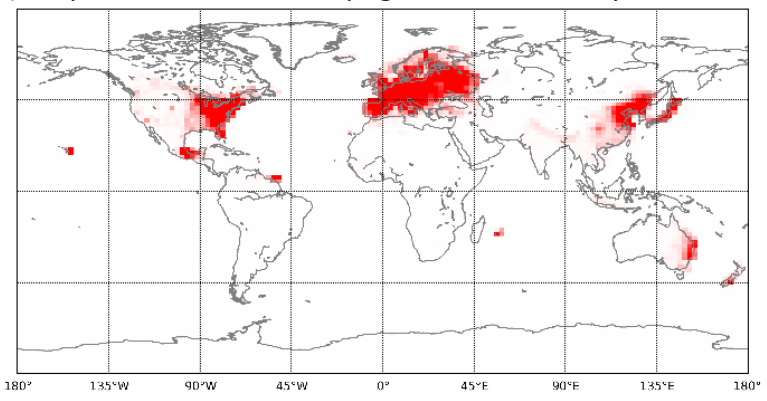

iii) July 2016 NDACC Anthropogenic CO sensitivity

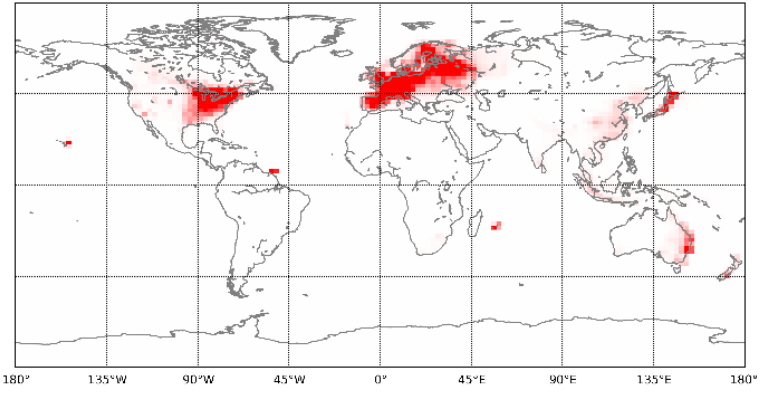

Figure 4.2.1.3: GEOS-Chem mean base surface anthropogenic CO sensitivity [unitless] for each month, May through August 2016. ii) June 2016 NDACC Anthropogenic CO sensitivity

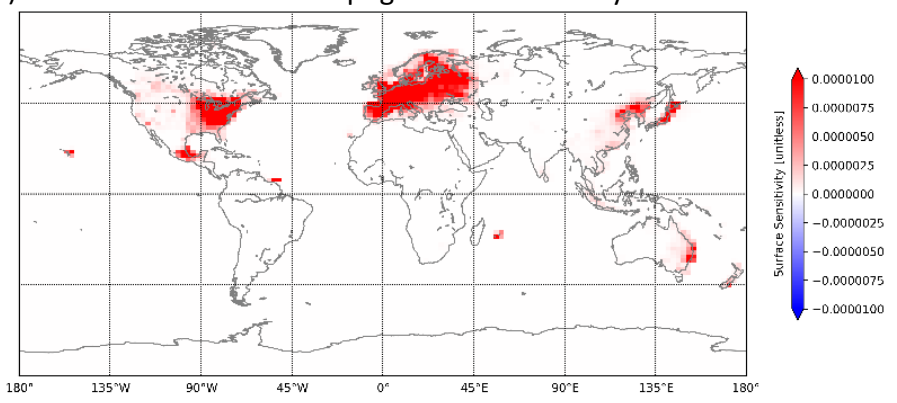

iv) August 2016 NDACC Anthropogenic CO sensitivity

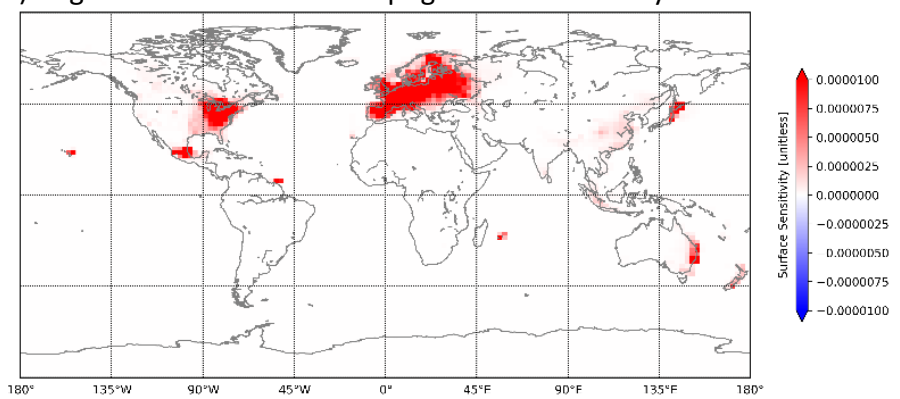

i) May 2016 NDACC Biomass Burning CO sensitivity

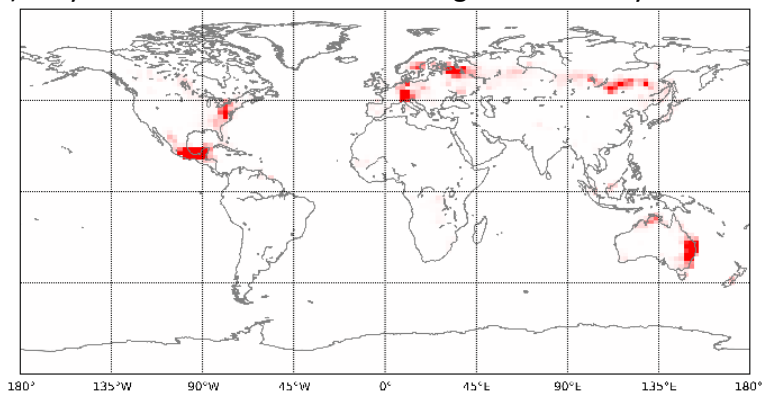

iii) July 2016 NDACC Biomass Burning CO sensitivity

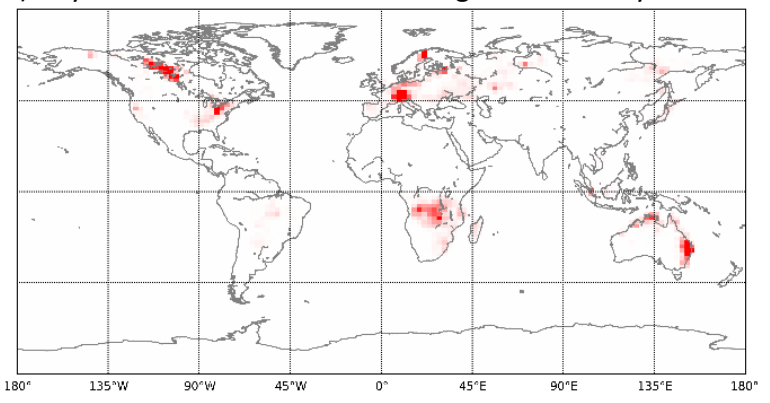

ii) June 2016 NDACC Biomass Burning CO sensitivity

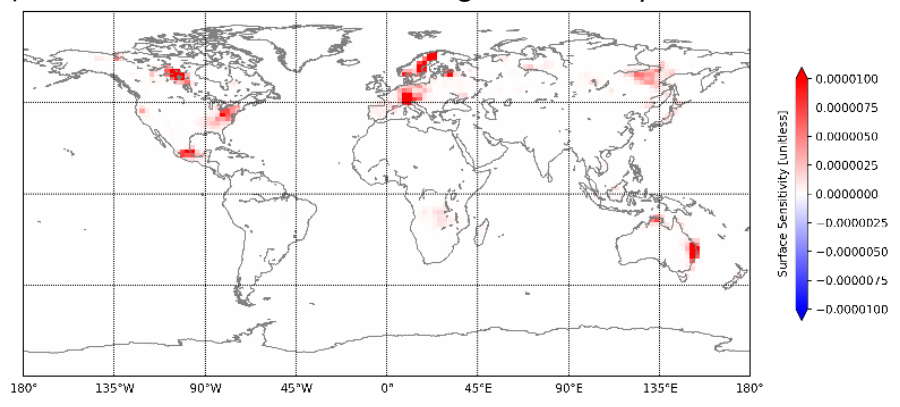

iv) August 2016 NDACC Biomass Burning CO sensitivity

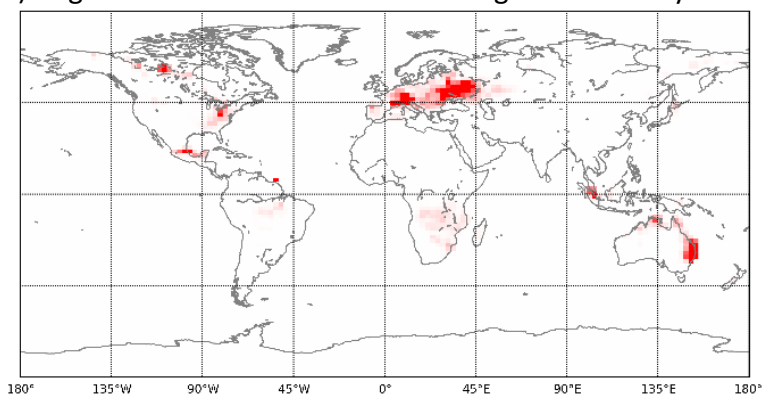

Figure 4.2.1.4: GEOS-Chem mean base surface biomass burning CO sensitivity [unitless] for each month, May through August 2016. 
i) May 2016 NDACC Biofuel CO sensitivity

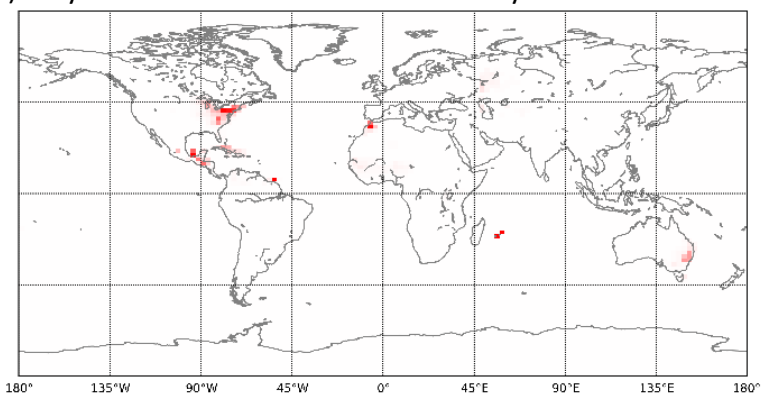

iii) July 2016 NDACC Biofuel CO sensitivity

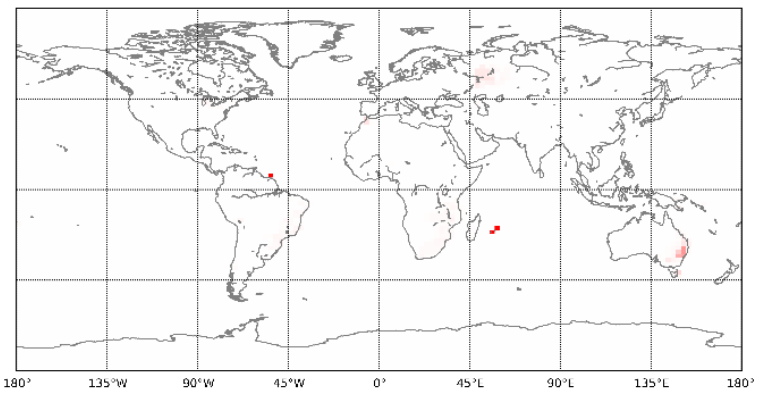

ii) June 2016 NDACC Biofuel CO sensitivity

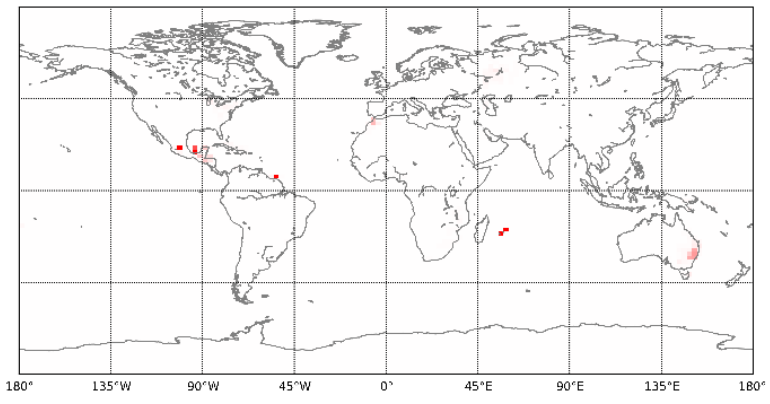

iv) August 2016 NDACC Biofuel CO sensitivity

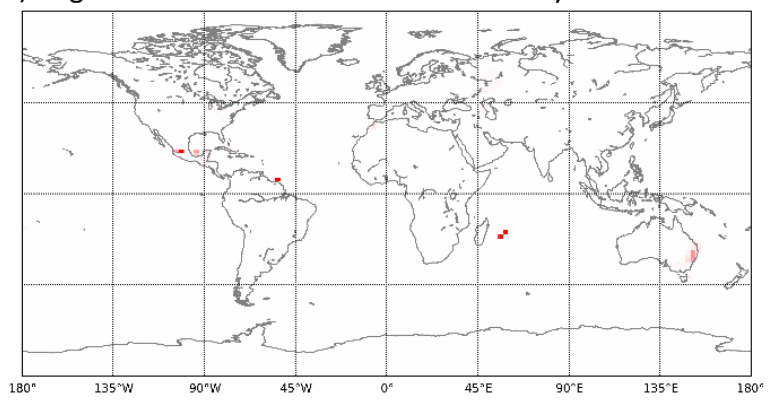

NDACC's biomass burning CO sensitivity (Figure 4.2.1.4) follows similar trends where sensitivities are strongest in proximity to NDACC station locations, and no sensitivity is found over polar stations. The difference being that biomass burning $\mathrm{CO}$ sensitivity is not restricted to NDACC station surroundings. Positive sensitivities appear in northern Canada, southern Africa, and eastern Russia in June, July, and August, which is linked to seasonal biomass burning emissions in the model. The NDACC adjoint is least sensitive to biofuel CO emissions, with emissions barely registering on the figure maps on the same scale and only appearing over select NDACC stations. NDACC global sensitivity across all CO emissions also do not appear to have much variability between months. 
i) May 2016 MOPITT Anthropogenic CO sensitivity

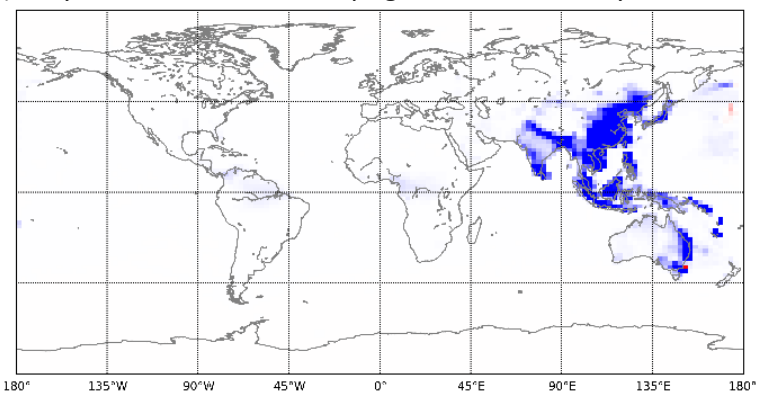

iii) July 2016 MOPITT Anthropogenic CO sensitivity

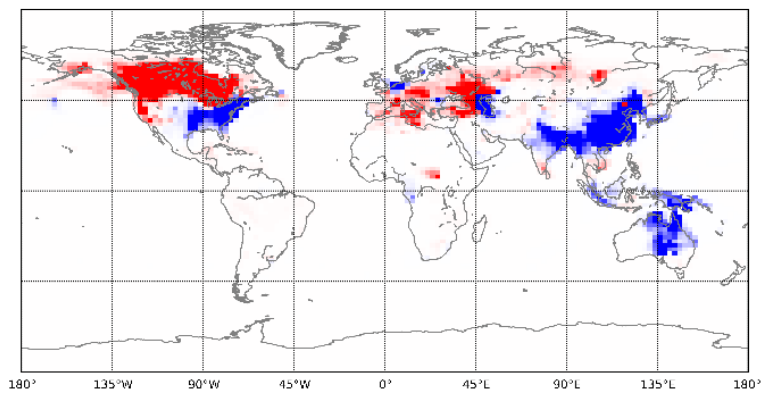

Figure 4.2.1.6: MOPITT model mean surface anthropogenic CO ii) June 2016 MOPITT Anthropogenic CO sensitivity

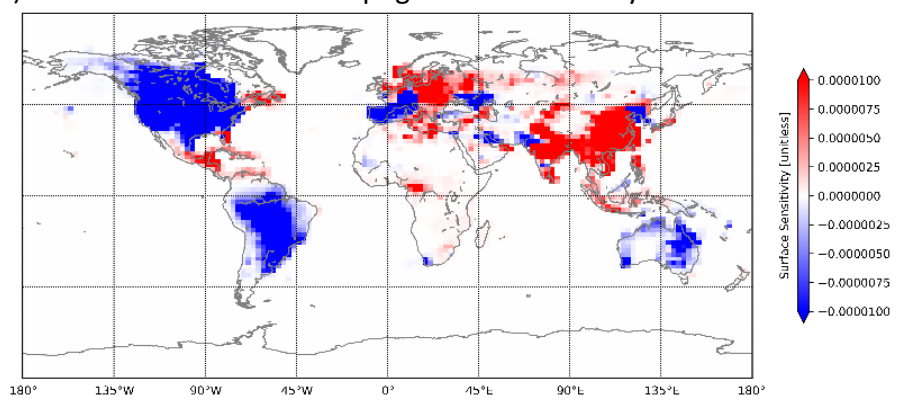

iv) August 2016 MOPITT Anthropogenic CO sensitivity

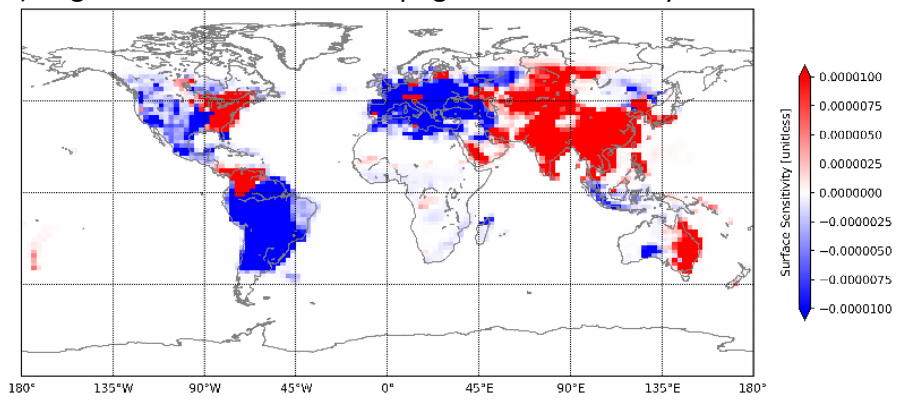

i) May 2016 MOPITT Biomass Burning CO sensitivity

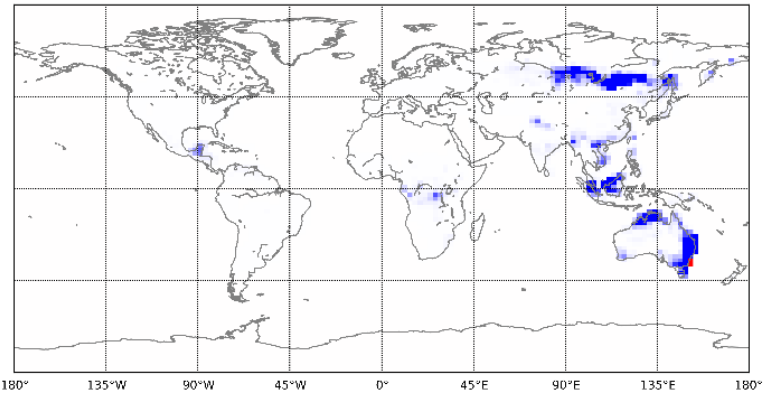

iii) July 2016 MOPITT Biomass Burning CO sensitivity

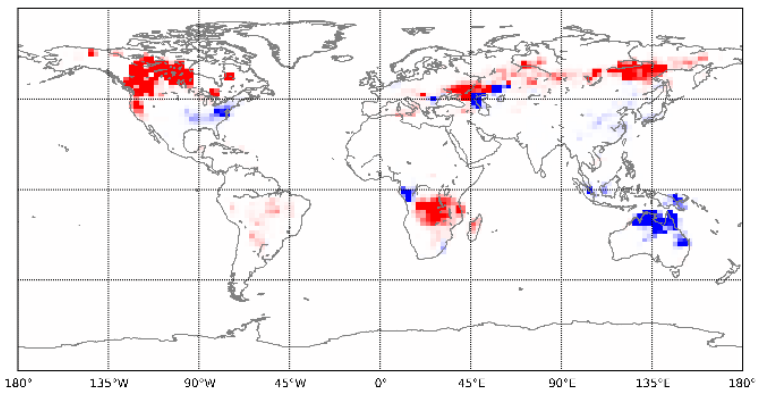

Figure 4.2.1.7: MOPITT model mean surface biomass burning CO sensitivity [unitless] for each month, May through August ii) June 2016 MOPITT Biomass Burning CO sensitivity

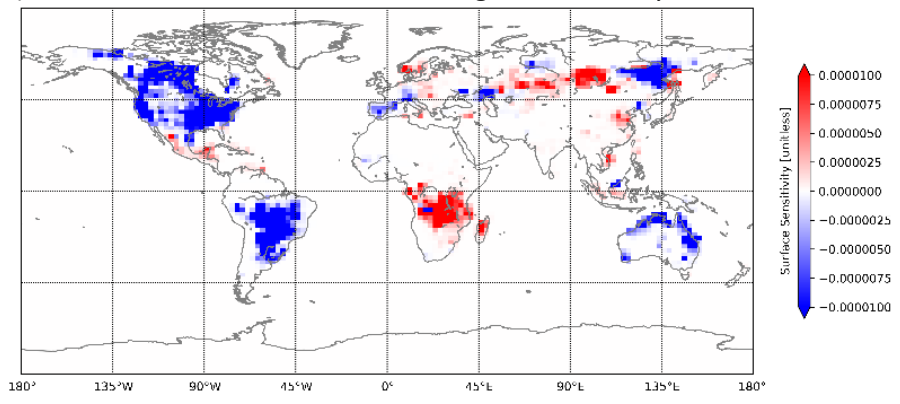

iv) August 2016 MOPITT Biomass Burning CO sensitivity

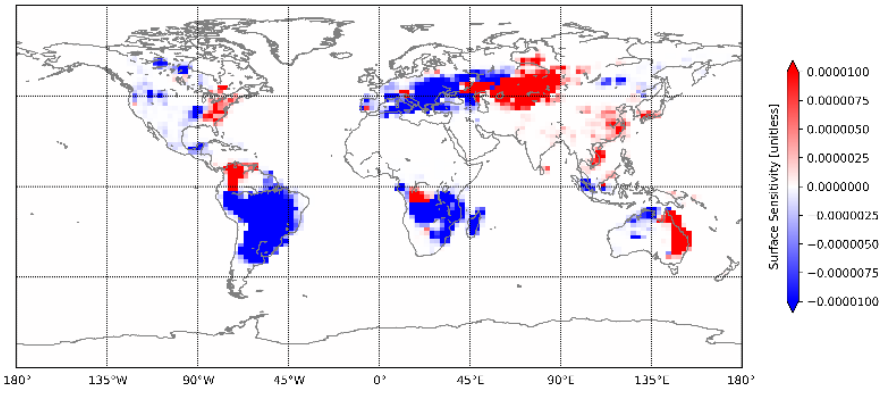
2016. 
i) May 2016 MOPITT Biofuel CO sensitivity

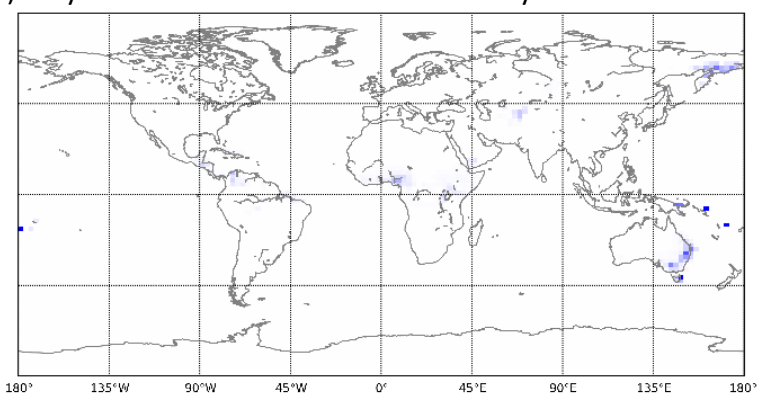

iii) July 2016 MOPITT Biofuel CO sensitivity

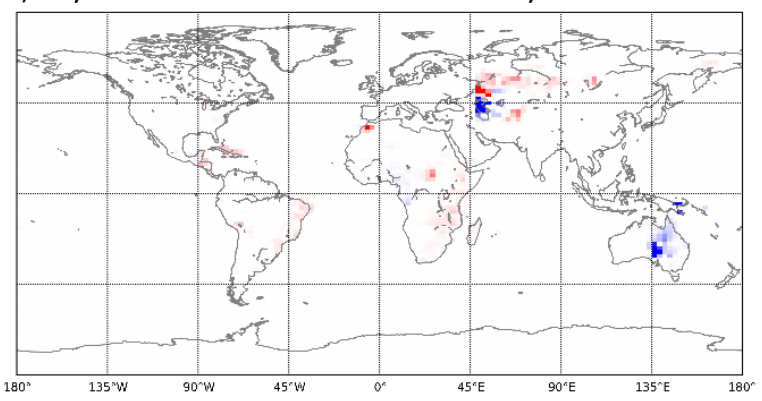

ii) June 2016 MOPITT Biofuel CO sensitivity

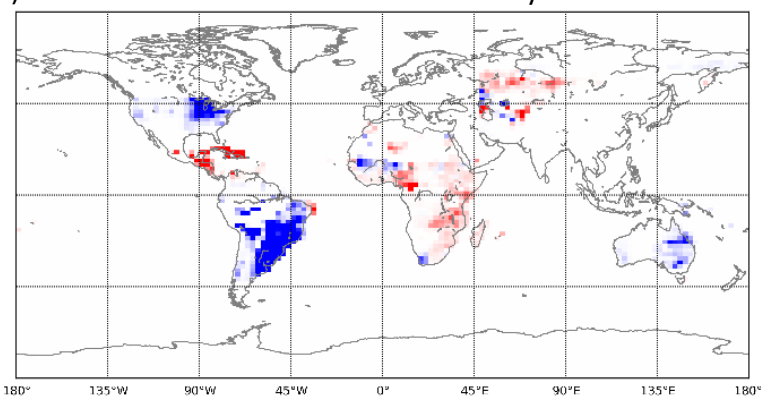

iv) August 2016 MOPITT Biofuel CO sensitivity

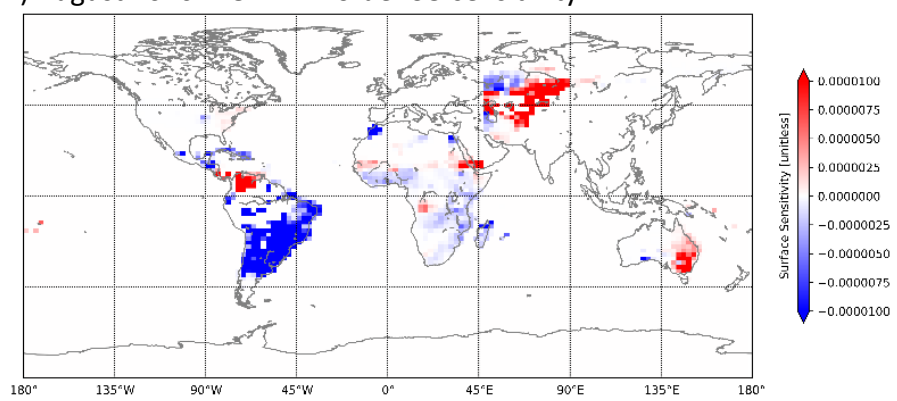

Figure 4.2.1.8: MOPITT model mean surface biofuel CO sensitivity [unitless] for each month, May through August 2016.

Like the NDACC adjoint CO emission sensitivities the MOPITT sensitivities are largest for anthropogenic $\mathrm{CO}$ emissions and smallest for biofuel emissions, and there is no sensitivity over the polar regions (Figures 4.2.1.6 to 4.2.1.8). Although, the spatial distribution of the MOPITT sensitivities appear proportional between anthropogenic, biomass burning, and biofuel CO emissions. Unlike the NDACC sensitivities, MOPITT has both positive and negative sensitivities, and is not restricted to NDACC station locations. For instance in May, the strongest negative sensitivity is found over east, south, and southeast Asia and Australia across all MOPITT CO sensitivity figures at varying magnitudes. In August, positive sensitivity is strongest over eastern North America, norther South America, eastern Australia, and most of Asia; and negative sensitivity is found over the remainder of North and South America, southern Africa, south-east Asia, Europe, and western Asia. Interestingly, sensitivities across all emission types appear strongest in June and August, rather than consecutive months. As these sensitivities provide 
direction to correct biases within the model, it makes sense that these observations have regional associations.

Although CO emission sensitivities for both NDACC and MOPITT in GEOS-Chem are small, they do have differences. These differences are shown in Appendix B.4 and indicate where MOPITT provides additional or contradictory information against NDACC. As mentioned in Section 3.3, the cost function for the NDACC station sensitivities is equivalent to CO concentration, where positive sensitivity represents an increase in model CO concentration due to increasing changes in emissions. Whereas the MOPITT CO emission sensitivities represent the model assimilation response, in which a positive sensitivity represents the model reducing CO concentrations to minimise the cost function for assimilation. Therefore, the figures in Appendix B.4 only show spatial differences in CO emission sensitivities, as the differences hold no numerical value. These spatial differences in sensitivities appear largest in June and August over land, like the MOPITT CO sensitivity figures.

\subsection{2 $\left[\mathrm{CH}_{4}+\mathrm{OH}\right]$ Reaction Sensitivity}

This section presents the NDACC and MOPITT sensitivity to the $\left[\mathrm{CH}_{4}+\mathrm{OH}\right]$ reaction rate, and their differences within the GEOS-Chem model. The reaction rate of $\mathrm{OH}$ with $\mathrm{CH}_{4}$ is used over $\mathrm{CO}$ because $\mathrm{CH}_{4}$ more stable within the monthly time frame. Therefore, methane's stable concentration yields more information on $\mathrm{OH}$ variability through time through the reaction rate. The $\left[\mathrm{CH}_{4}+\mathrm{OH}\right]$ reaction rate sensitivity represents the direction and magnitude of how the model would correct the reaction rate values to fit the observations. Here, a positive sensitivity represents a model response where the cost function is increasing due to increasing 
changes in $\left[\mathrm{CH}_{4}+\mathrm{OH}\right]$ reaction rate. Negative sensitivity represents a model response where the cost function is decreasing due to increasing changes in $\left[\mathrm{CH}_{4}+\mathrm{OH}\right]$ reaction rate.

Like the CO emission sensitivities, the NDACC and MOPITT reaction rate sensitivities do not have the same representation. A positive NDACC sensitivity represents an increase in model CO concentrations due to increasing changes in the $[\mathrm{CH} 4+\mathrm{OH}]$ reaction rate. For the MOPIT assimilation, a positive sensitivity tells the model to reduce the reaction rate to closer match $\mathrm{CO}$ observations. Again, a negative sensitivity indicates the opposite, where the model response will be to increase the model reaction rate. 
i) May $2016 \mathrm{NDACC}\left[\mathrm{CH}_{4}+\mathrm{OH}\right]$ sensitivity
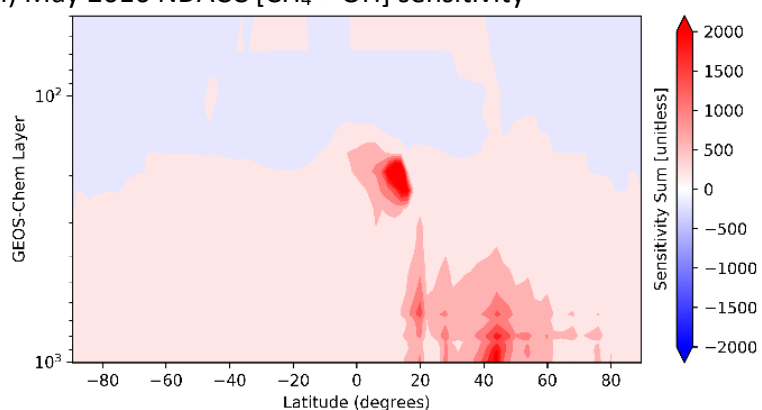

ii) June $2016 \mathrm{NDACC}\left[\mathrm{CH}_{4}+\mathrm{OH}\right]$ sensitivity

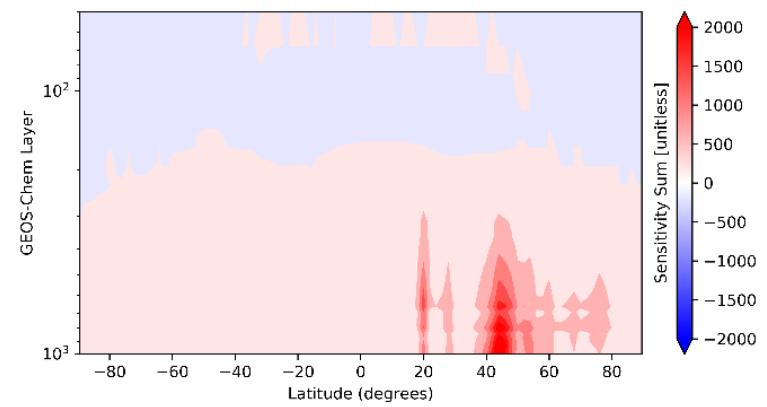

iii) July 2016 NDACC $\left[\mathrm{CH}_{4}+\mathrm{OH}\right]$ sensitivity

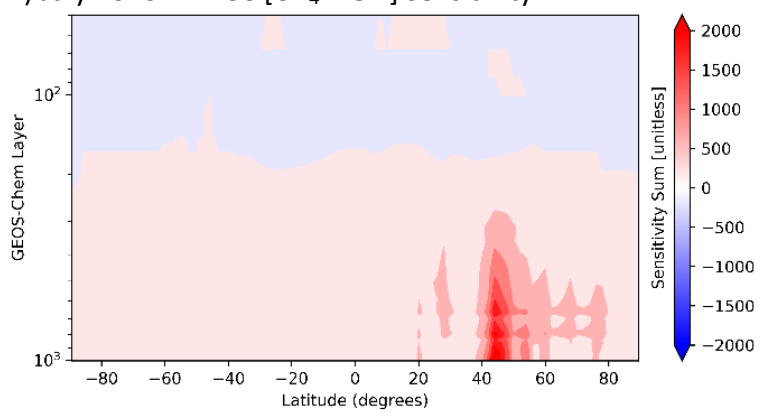

iv) August $2016 \mathrm{NDACC}\left[\mathrm{CH}_{4}+\mathrm{OH}\right]$ sensitivity

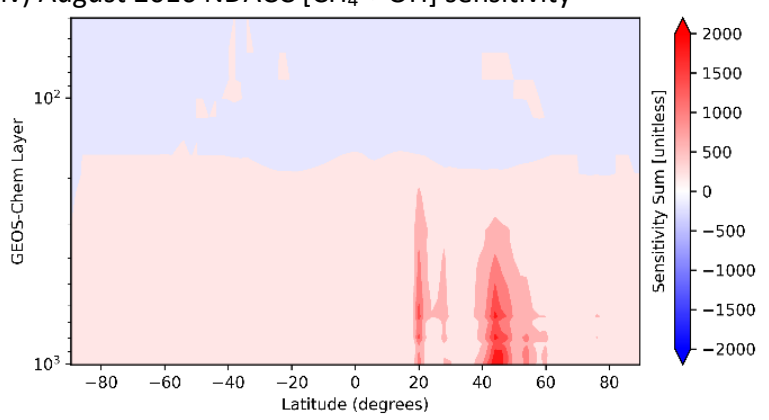

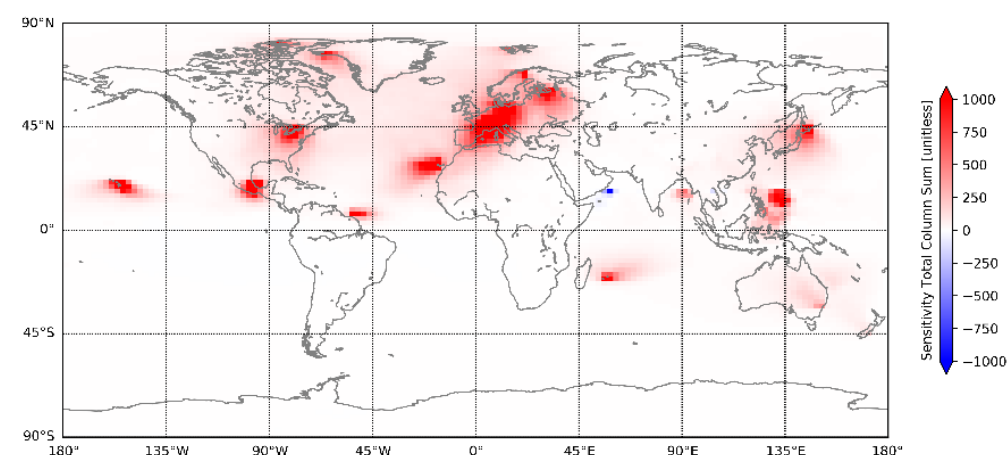
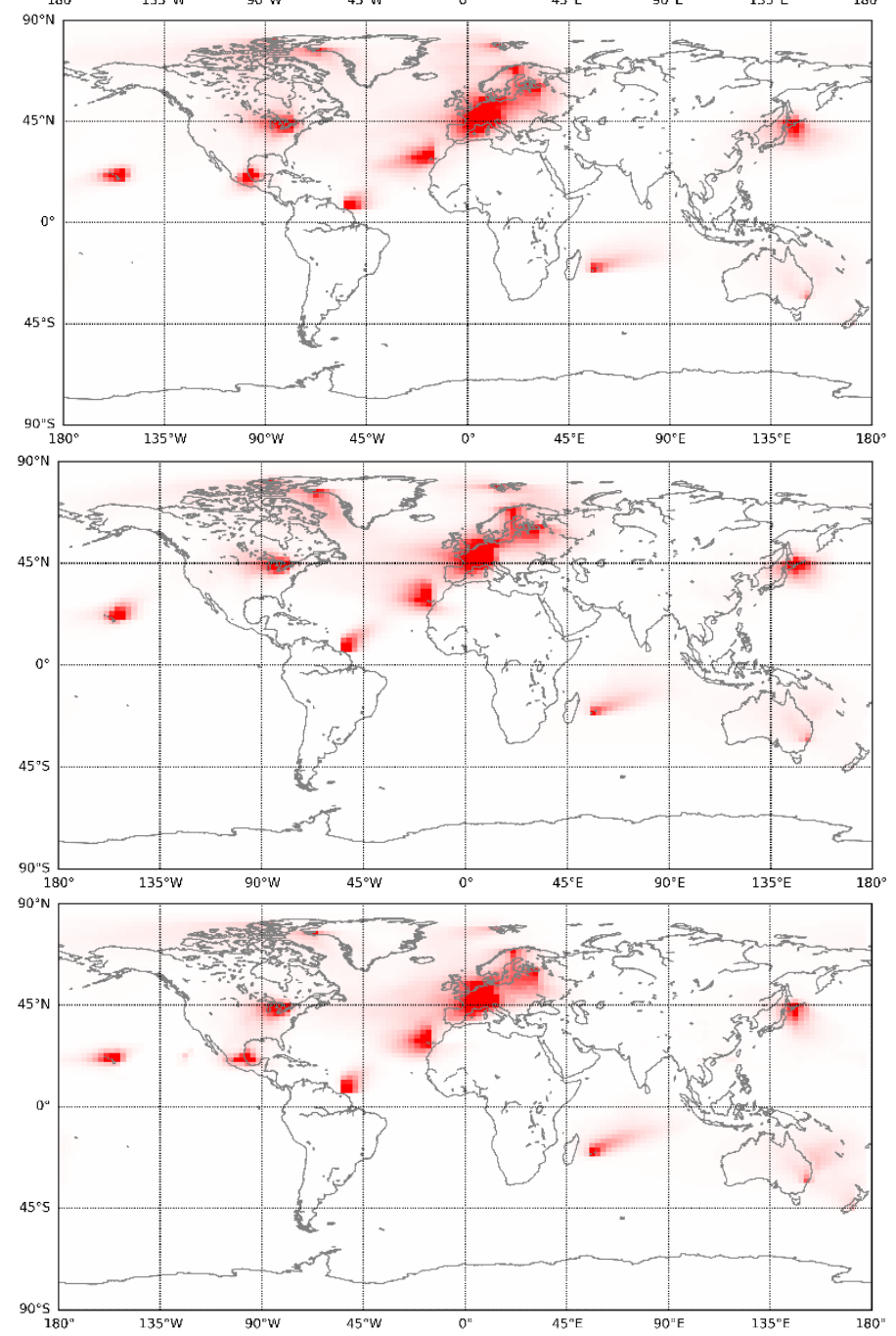

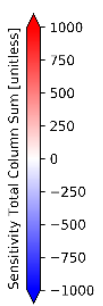

Figure 4.2.2.1: NDACC mean global $\left[\mathrm{CH}_{4}+\mathrm{OH}\right]$ reaction sensitivity [unitless] for each month, May through August 2016.

The NDACC $\left[\mathrm{CH}_{4}+\mathrm{OH}\right]$ reaction sensitivity is largely positive throughout the troposphere between the surface and $110 \mathrm{hPa}$, with a slight negative sensitivity within the 
upper troposphere above $110 \mathrm{hPa}$ (Figure 4.2.2.1). In all the left-hand figures, a distinguishable second cut-off between positive and negative sensitivity can be seen around $50 \mathrm{hPa}$. This is the $38^{\text {th }}$ layer within GEOS-Chem, where GEOS-Chem does not model OH. This line of separation may be generated from the fixed $\mathrm{OH}$ level and variable tropopause height generated within the model. Like the CO emission sensitivity figures in Section 4.2.1, NDACC sensitivity to the $\left[\mathrm{CH}_{4}+\right.$ $\mathrm{OH}$ ] reaction is limited to the locations of the NDACC stations. Positive NDACC reaction sensitivity is strong in the Northern Hemisphere directly over each station in the lower to midtroposphere, mostly between $20^{\circ}$ and $60^{\circ}$ latitude. The strongest total sensitivity occurs in May, where additional positive mid-troposphere sensitivity is found between $0^{\circ}$ and $20^{\circ}$ latitude.

The MOPITT $\left[\mathrm{CH}_{4}+\mathrm{OH}\right]$ reaction sensitivity is largely negative throughout the background troposphere between the surface and $110 \mathrm{hPa}$, with a slight positive sensitivity within the upper troposphere above $110 \mathrm{hPa}$ in May (Figure 4.2.2.1). Almost an inverse of the NDACC sensitivity, including the strong sensitivity pocket in the mid-troposphere between $0^{\circ}$ and $10^{\circ}$ latitude. In all the left-hand figures, general lines of separation between sensitivities can bee seen at $110 \mathrm{hPa}$ and $50 \mathrm{hPa}$, although less distinguishable than the NDACC sensitivities. Between 110 and $50 \mathrm{hPa}$, the summed sensitivity generally appears to separate from the sensitivity directly beneath $110 \mathrm{hPa}$. 
i) May 2016 MOPITT $\left[\mathrm{CH}_{4}+\mathrm{OH}\right]$ sensitivity

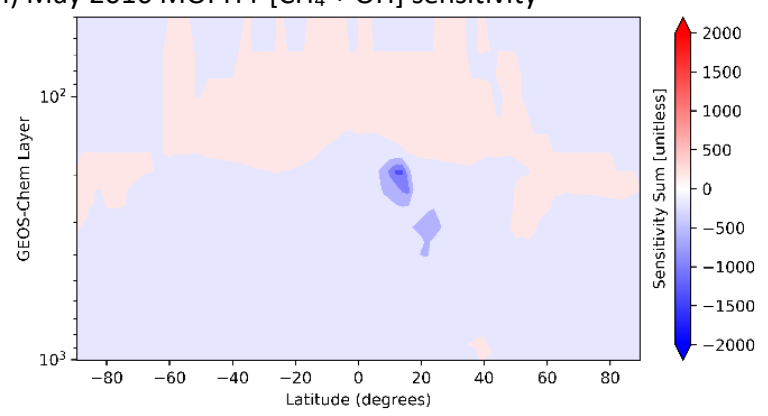

ii) June 2016 MOPITT $\left[\mathrm{CH}_{4}+\mathrm{OH}\right]$ sensitivity

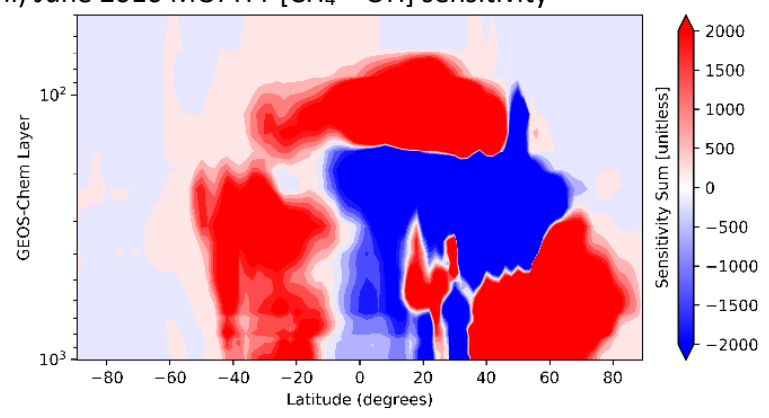

iii) July 2016 MOPITT $\left[\mathrm{CH}_{4}+\mathrm{OH}\right]$ sensitivity

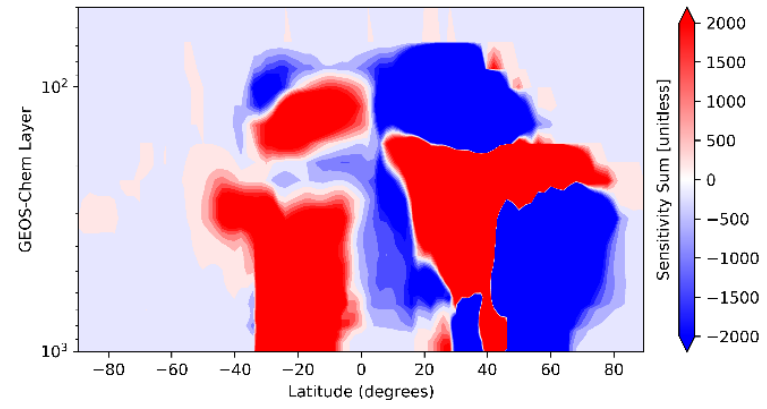

iv) August $2016 \mathrm{MOPITT}\left[\mathrm{CH}_{4}+\mathrm{OH}\right]$ sensitivity

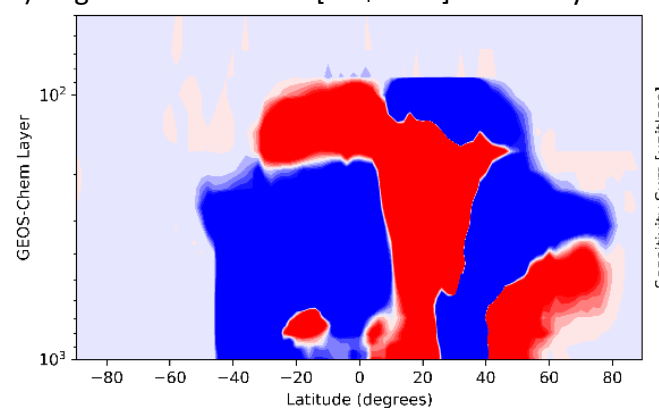

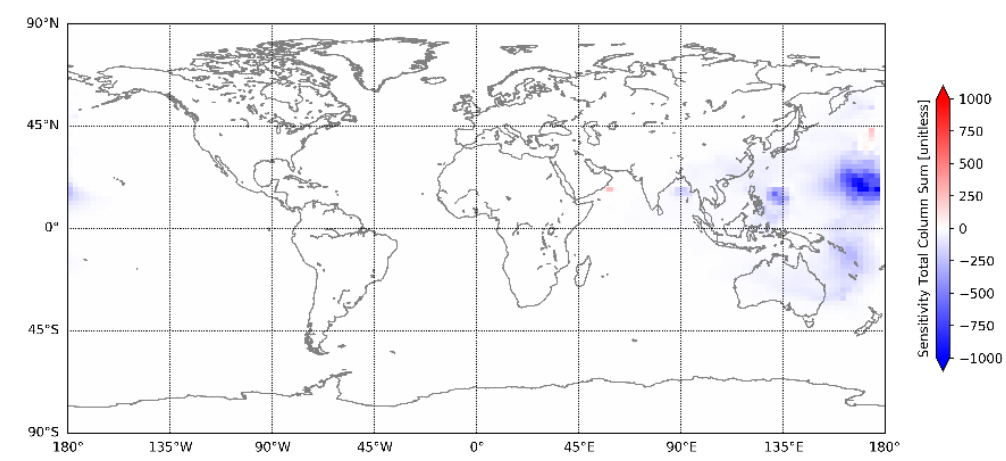
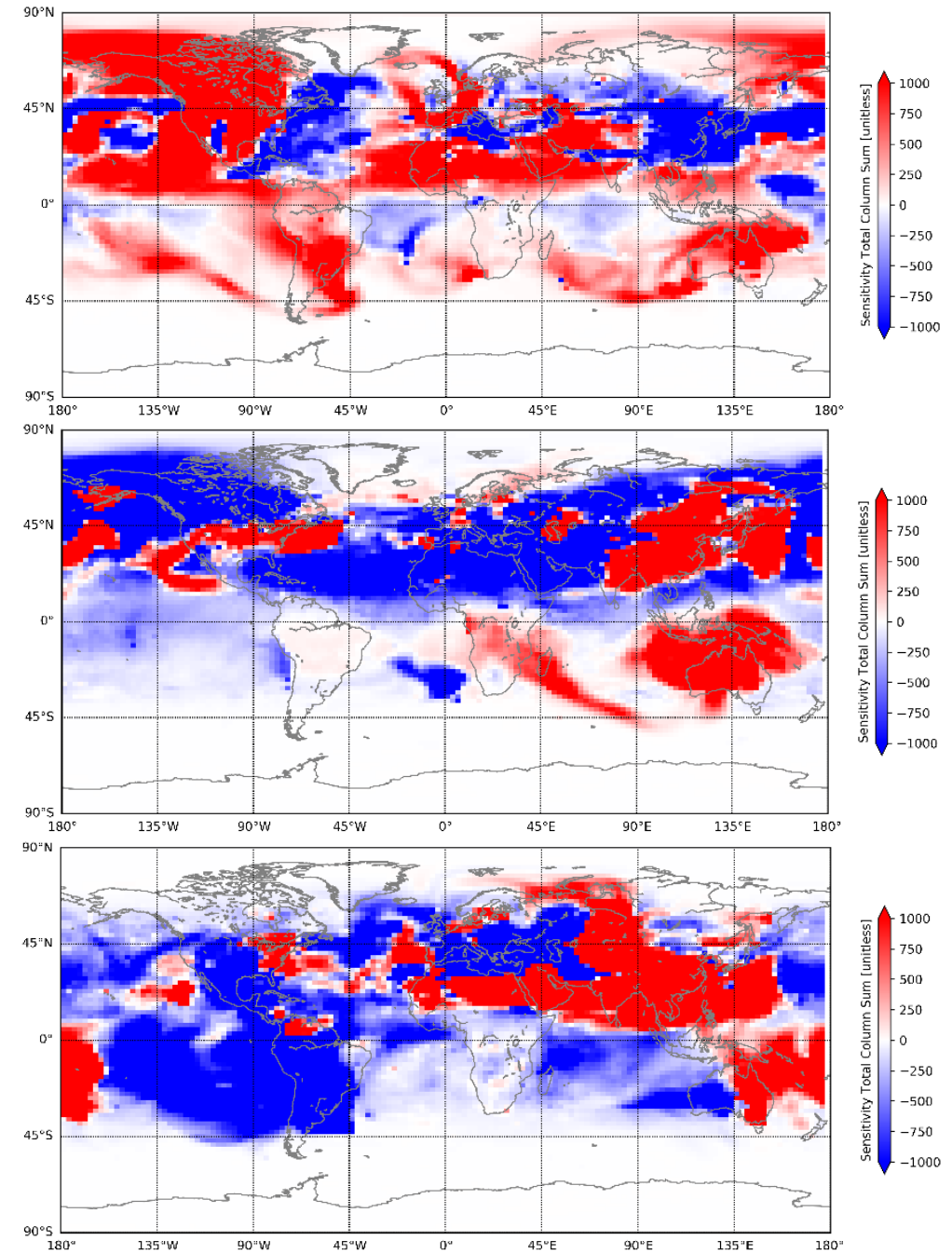

1000
-750
-500
-250
0
-250
-500
-750
-1000

Figure 4.2.2.2: MOPITT mean global $\left[\mathrm{CH}_{4}+\mathrm{OH}\right.$ ] reaction sensitivity [unitless] for each month, May through August 2016. 
June through August, the MOPITT reaction sensitivities appear to have a general sensitivity division along the equator. In June, positive sensitivity dominates the Southern Hemisphere between approximately $-45^{\circ}$ and $-10^{\circ}$ latitude. In July, this dominating positive sensitivity shifts slightly north between $-40^{\circ}$ and $0^{\circ}$ latitude, and between Africa and Australasia. In August, negative sensitivity dominates the Southern Hemisphere beneath 110 $\mathrm{hPa}$, with the positive sensitivity diminishing over eastern Australasia and part of the Pacific. In the Northern Hemisphere, the positive and negative sensitivities are more mixed than within the Southern Hemisphere from June through August. These strong positive and negative sensitivities appear to have the largest horizontal extent in June from approximately $-10^{\circ}$ to $80^{\circ}$ latitude, and smallest extent in August between $0^{\circ}$ and $60^{\circ}$ in the lower troposphere.

Figure B.5.1 in Appendix B5 shows the MOPITT $\left[\mathrm{CH}_{4}+\mathrm{OH}\right]$ reaction sensitivities minus the NDACC reaction sensitivities. Like the figures in Appendix B.4, positive and negative values only reveal spatial differences rather than numerical differences. Here, vertical differences in sensitivity appear to be governed by NDACC reaction sensitivities, with greatest differences occurring over NDACC stations within the Northern Hemisphere between $20^{\circ}$ and $60^{\circ}$ latitude. Sensitivity differences are largest in the Northern Hemisphere, with an approximate separation at the equator appearing similar to the MOPITT sensitivity horizontal distribution (Figure 4.2.2.2). 


\subsubsection{Discussion}

CO abundance and distribution between the GEOS-Chem base and MOPITT integrated model outputs are similar (Figure 4.2.1.1). After one adjoint iteration, the MOPITT integrated model shows a strong $\mathrm{CO}$ agreement throughout all four months with strongest agreement in May and June (Figure 4.2.1.2). Minor differences appear to be a result of random calculation/observation differences within the Southern Hemisphere for May, and propagated error in the upper troposphere and lower stratosphere stemming from the modeled moving tropopause in June. In July and August, the moving tropopause appears to increase the difference between the modeled $\mathrm{CO}$ outputs. The moving tropopause may be introducing propagating model error. It also appears that the MOPITT integrated output has slightly greater CO concentrations within the upper troposphere between $60^{\circ}$ and $90^{\circ}$ latitude, and the lower to mid-troposphere in the Southern Hemisphere. These growing differences may be indicative of an underestimation of $\mathrm{CO}$ within the GOES-Chem base model for these sections towards August. It may also be attributed to bias in modeled transportation, as horizontal differences appear in three bands in August around $45^{\circ}$ latitude North and South.

Comparing CO emission sensitivities (Appendix B.4), NDACC's sensitivities are all positive and reasonably consistent. The positive sensitivity represents an increase in model CO concentration due to increasing changes in emissions. These sensitivities reveal that an assimilation of NDACC station CO observations would be generally restricted to their geographical locations, and as a result introduce a Northern Hemisphere bias where most stations are located. Although, there appears to be greater sensitivity to biomass burning emission transportation, marginally widening $\mathrm{CO}$ emission sensitivity beyond immediate 
geographical station locations. They also reveal that the integration of polar stations would not impact the modeled $\mathrm{CO}$ output from $\mathrm{CO}$ emissions, as no $\mathrm{CO}$ emission sensitivity is shown in these regions over these four months.

Like NDACC, the MOPITT assimilation has not sensitivity to polar regions and is not impacted by anthropogenic, biomass burning, or biofuel CO emissions. Unlike NDACC, the MOPITT assimilated $\mathrm{CO}$ emission sensitivities are both positive and negative, with greater variability than the NDACC sensitivities. A positive sensitivity represents the model reducing CO concentrations in response to increasing changes in $\mathrm{CO}$ emissions, to minimise the cost function for assimilation. A negative sensitivity represents the model increasing CO concentrations in response to increasing changes in $\mathrm{CO}$ emissions, to minimise the cost function for assimilation. These positive and negative sensitivity pockets change sign in each month, although they appear over the same geographical regions with different magnitudes. This may be indicative of regional dependencies and/or differences between the model and MOPITT. Sensitivities also appear strongest in non-consecutive months (June and August). The change in sign and higher sensitivities in June and August may be due to the monthly time scale used, as the same region may be biased high one month compared to the next. More assimilation iterations may be able to confirm regional and temporal relationships, as a single iteration only provides a first look into model assimilation responses before the cost function in minimised.

This global sensitivity coverage demonstrates MOPITT's ability observe and correct global CO emissions within the model, rather than being constrained to geographical locations like NDACC. This is also reflected in the differences between the CO emission sensitivity outputs where the differences appear similar to MOPITT sensitivity outputs. The greatest differences 
are between the anthropogenic $\mathrm{CO}$ emission sensitivities where differences lie over land and oceans (Appendix B.4, Figure B.4.1). For example, MOPITT could encompass anthropogenic transportation and oil \& gas operation emissions over sea. MOPITT's global coverage therefore has greater potential to constrain global $\mathrm{CO}$ emissions than the NDACC network, accentuating the importance of satellite assimilation. This has a larger impact on calculating global CO and therefore $\mathrm{OH}$.

Looking at the $\left[\mathrm{CH}_{4}+\mathrm{OH}\right]$ reaction rate sensitivities (Figures 4.2.2.1 to 4.2.2.2), NDACC's sensitivities are again mostly positive and consistent within the troposphere with strongest sensitivities directly over NDACC stations. Positive sensitivities indicate an increase in model CO concentrations due to increasing changes in $[\mathrm{CH} 4+\mathrm{OH}]$ reaction rate. Unlike the $\mathrm{CO}$ emission sensitivities, reaction rate sensitivities are observed over stations towards the North Pole. This implies that northern polar $\mathrm{CO}$ concentrations are influenced by $\left[\mathrm{CH}_{4}+\mathrm{OH}\right]$ reactions in this region over these four months. It is logical that the Arrival Heights would not show reaction sensitivity during this time of year due to lack of sunlight, and therefore $\mathrm{OH}$.

Since $\mathrm{OH}$ is only modeled within the first 38 layers in GEOS-Chem, it is also sensible that the positive reaction sensitivity is limited to below approximately $50 \mathrm{hPa}$. However, there are positive sensitivities above $50 \mathrm{hPa}$. This implies $\mathrm{CO}$ transport from the stratosphere influencing $\mathrm{OH}$, but it is odd that the dividing line is so clear and straight. Again, this may be a result of the modeled variable tropopause introducing error. Interestingly, the upper troposphere between $110 \mathrm{hPa}$ and $50 \mathrm{hPa}$ contains a minor negative sensitivity, including in the tropical region where the tropopause is higher. Negative sensitivities indicate a decrease in CO concentrations due to increasing changes in $[\mathrm{CH} 4+\mathrm{OH}]$ reaction rate. 
The MOPITT assimilation $\left[\mathrm{CH}_{4}+\mathrm{OH}\right]$ sensitivity output shows greater spatial sensitivity than NDACC, and greater sensitivity in all four months except for May. May exhibits the lowest MOPITT reaction sensitivity with most of the troposphere having a slight negative sensitivity, strongest over the West Pacific. Both the NDACC and MOPITT sensitivities show a strong midtroposphere grouping in May at this location. It is uncertain why this appears in both sensitivity plots but is likely attributed to a common element in the model. Further MOPITT assimilation through additional adjoint iterations may correct and remove this spot, should it be a product of modeled assumptions or error.

In June, July, and August, the MOPITT $\left[\mathrm{CH}_{4}+\mathrm{OH}\right]$ reaction rate sensitivity magnitude and distribution greatly differ from NDACC's. Its sensitivity ranges between $-50^{\circ}$ and $90^{\circ}$ latitude over these months, with greatest sensitivity breadth within the free troposphere. Like the CO emission sensitivities, the reaction rate sensitivities appear to have shifting regional positive and negative groupings. There also appears to be a sensitivity separation line around $110 \mathrm{hPa}$, dividing sensitivities within the upper troposphere where the tropopause is highest. Another sensitivity separation is visible in the low to mid-troposphere between the Northern and Southern Hemisphere. Together, these separation lines may show that $\left[\mathrm{CH}_{4}+\mathrm{OH}\right]$ chemistry is largely divided between the Northern and Southern Hemisphere but do mix within the upper troposphere where the tropopause exceeds $110 \mathrm{hPa}$. However, these divisions may only exist for the first MOPITT assimilation iteration. Further iterations may further adjust tropospheric chemistry and transport, removing these observed divisions. Without further iterations it is difficult to determine significance of the reaction rate sensitivities, although it does show the 
MOPITT CO assimilation's initial impact on the modeled $\left[\mathrm{CH}_{4}+\mathrm{OH}\right]$ chemistry, and therefore global $\mathrm{OH}$.

The differences in reaction rate sensitivity coverage again demonstrates MOPITT's ability observe and reflect global chemistry within model, rather than being constrained to geographical locations like NDACC. In Figure B.5.1, the greatest vertical differences between the two $\left[\mathrm{CH}_{4}+\mathrm{OH}\right]$ sensitivity outputs span over the NDACC latitudes. This may be reflective of potential constant temporal NDACC coverage at these latitudes, compared to MOPITT observations every few days over these stations. Although an assimilation of the NDACC network has potential to provide a better vertical $\mathrm{OH}$ resolution from greater vertical reaction rate sensitivity, this is a selective area of global atmosphere and would highly depend on individual station observation frequency. Horizontally the greatest differences are representative of the MOPITT sensitivities, apart for May reflecting NDACC sensitivities. Again, this demonstrates MOPITT's greater global coverage. Therefore, the MOPITT CO assimilation would provide better global $\mathrm{OH}$ outputs with a less selective sensitivity to $\left[\mathrm{CH}_{4}+\mathrm{OH}\right]$ chemistry than NDACC.

Together, the $\mathrm{CO}$ emission and $\left[\mathrm{CH}_{4}+\mathrm{OH}\right]$ reaction rate sensitivities reveal observing platform measurement characteristics in calculating global $\mathrm{OH}$. The assimilation of MOPITT CO observations into the GEOS-Chem model yield vastly different sensitivities than NDACC observations would, accentuating the necessity for satellite integrated models to determine $\mathrm{OH}$. With only one MOPITT adjoint iteration, the $\mathrm{CO}$ abundance and emission sensitivities may not be fully established or fully reflective of MOPITT observations. This is likely why only minimal differences are found between the GEOS-Chem base and MOPITT integrated CO 
outputs. Although, the single iteration does reveal beginnings of spatial differences through their sensitivities.

For future studies, multiple adjoint iterations of MOPITT CO observations may reveal larger differences against the CO base GEOS-Chem outputs. Observed increasing differences in the upper troposphere are likely propagating error caused by the modeled variable tropopause. This may also be a cause to why May sensitivity magnitudes and CO differences are significantly smaller than in August. Therefore, it is recommended that the multiple iterations should be run with a fixed tropopause. The assimilation of satellite $\mathrm{CO}$ observations would provide significantly better spatial and temporal coverage to constrain $\mathrm{OH}$ than ground monitoring station networks such as NDACC.

Further studies with CrIS and TROPOMI integrated CO observations would be expected to have similar observations to the MOPITT integrated model. Even though the CrIS CLIMCAPS observations are less sensitive to the boundary layer, its higher profile resolution may reveal greater $\left[\mathrm{CH}_{4}+\mathrm{OH}\right]$ reaction rate sensitivity in the upper and mid-troposphere - where MOPITT sensitivities are greatest. CrIS would therefore be able to better constrain $\mathrm{CO}$ loss and $\mathrm{OH}$ concentrations but not $\mathrm{CO}$ emissions. TROPOMI's higher total column resolution and greater $\mathrm{CO}$ emission sensitivities at the surface would be able to better constrain CO emissions, but not atmospheric CO loss. Using both satellites together in the Joint CrIS-TROPOMI product, these greater sensitivity resolutions would have a greater impact on determining a better constrained global OH distribution compared to MOPITT and may also reveal regionally defined trends. 


\subsection{Part 3: $\mathrm{OH}$ evaluation}

Part 3 contains observations and evaluations between the global $\mathrm{OH}$ distribution from the GEOS-Chem base model, MOPITT assimilated model after one iteration, and past literature. Section 4.3.1 presents modeled global $\mathrm{OH}$ spatial distribution, and compares weighted $[\mathrm{OH}]_{\mathrm{GM}}$ and $\mathrm{NH} / \mathrm{SH}$ interhemispheric ratios. These observations are then discussed in Section 4.3.2. The $[\mathrm{OH}]_{\mathrm{GM}}$ and $\mathrm{NH} / \mathrm{SH}$ ratio outputs produced by both the GEOS-Chem base and MOPITT show little difference and are greater than referenced values in Table 2.3.1.1. Although they do reveal questionable differences along the tropopause, which increase May through August suggesting tropopause model error. Evaluations of CrIS and TROPOMI model assimilations are not included due to time constraints but are recommended for future studies.

\subsubsection{OH distribution}

The global OH distribution between the GEOS-Chem base model and the MOPITT assimilation model are nearly indistinguishable from each other like the CO model outputs in Section 4.2.1. Calculated weighted $[\mathrm{OH}]_{\mathrm{GM}}$ and $\mathrm{NH} / \mathrm{SH}$ interhemispheric ratios below $100 \mathrm{hPa}$ for each month are summarised in Table 4.3.1.1 below. Figures 4.3.1.1 and 4.3.1.2 show these values over time, along with the differences between the GEOS-Chem base model and the MOPITT assimilation model outputs.

Airmass weighted $[\mathrm{OH}]_{\mathrm{GM}}$ by both the GEOS-Chem base model and MOPITT assimilation model range from approximately $13.02 \times 10^{5}$ to $14.38 \times 10^{5}$ molecules $/ \mathrm{cm}^{3}$ (Table 4.3.1.1 and Figure 4.3.1.1). The $[\mathrm{OH}]_{\mathrm{GM}}$ and $\mathrm{OH}$ spatial differences between the base GEOS-Chem and MOPITT outputs are minimal but are greatest in July (Figure 4.3.1.1). As discussed in Section 
3.1.6, this study's $[\mathrm{OH}]_{\mathrm{GM}}$ range is greater than the average v8-02-01 GEOS-Chem model and most literature in Table 2.3.1.1. These averages are annual averages over different years and are not necessarily representative for May through August. Greater Northern Hemisphere summer $[\mathrm{OH}]_{\mathrm{GM}}$ is expected and is reflected in past literature presented in Table 2.3.1.1. For example, X. Zhang et. al. (2019) report a CO mass weighted [OH] $]_{\mathrm{GM}}$ of $13.6 \times 10^{5}$ in July to be approximately $15.25 \%$ greater than in November 2010. Their MSA mass weighted July $[\mathrm{OH}]_{\mathrm{GM}}$ of $12.8 \times 10^{5}$ is $21.9 \%$ greater than in November. Airmass weighted [OH] $]_{G M}$ by both the GEOSChem base model and MOPITT assimilation model range are still larger than these July 2010 values, even when incorporating the decadal average IAV by Turner et. al. (2018).

The mean airmass weighted NH/SH ratio ranges from 1.77 to 2.18 (Table 4.3.1.1 and Figure 4.3.1.2). This is high compared to ratios presented in literature, although most journal studies are an annual average rather than monthly averages within the summer. Patra et al. (2014) finds the annual average should be close to one, but notes that NH winters experience lower $\mathrm{OH}$ and $\mathrm{NH}$ summers experience higher $\mathrm{OH}$. With an expected $\mathrm{OH}$ shift north within the summer (see Section 2.2.1), it is logical that the $\mathrm{NH} / \mathrm{SH}$ ratio would be higher in the Northern Hemisphere summer months. The ratio difference between the base GEOS-Chem and MOPITT outputs are minimal but are again greatest in July (Figure 4.3.1.2). 
Table 4.3.1.1: Global mean $\mathrm{OH}$ (molecules/ $\mathrm{cm}^{3}$ ) and mean $\mathrm{NH} / \mathrm{SH}$ ratio for the GEOS-Chem adjoint base model output for each month, May through August 2016, below 100 hPa. Each global mean OH and NH/SH ratio are provided in airmass, CO, and volume weighted values.

\begin{tabular}{|c|c|c|c|}
\cline { 2 - 4 } \multicolumn{1}{c|}{} & \multicolumn{3}{c|}{ Global mean OH $\left(\right.$ molecules $/ \mathrm{cm}^{3}$ ) } \\
\hline Month & Airmass weighted & CO weighted & volume weighted \\
\hline May & 1302567 & 1434651 & 1244772 \\
\hline June & 1385787 & 1534840 & 1320330 \\
\hline July & 1438415 & 1569526 & 1369853 \\
\hline August & 1430792 & 1544331 & 1372157 \\
\hline & \multicolumn{3}{|c|}{ Mean NH/SH Ratio } \\
\hline May & 2.31 & 2.16 & 2.09 \\
\hline June & 2.99 & 2.78 & 2.70 \\
\hline July & 3.06 & 2.83 & 2.84 \\
\hline August & 2.34 & 2.17 & 2.23 \\
\hline
\end{tabular}

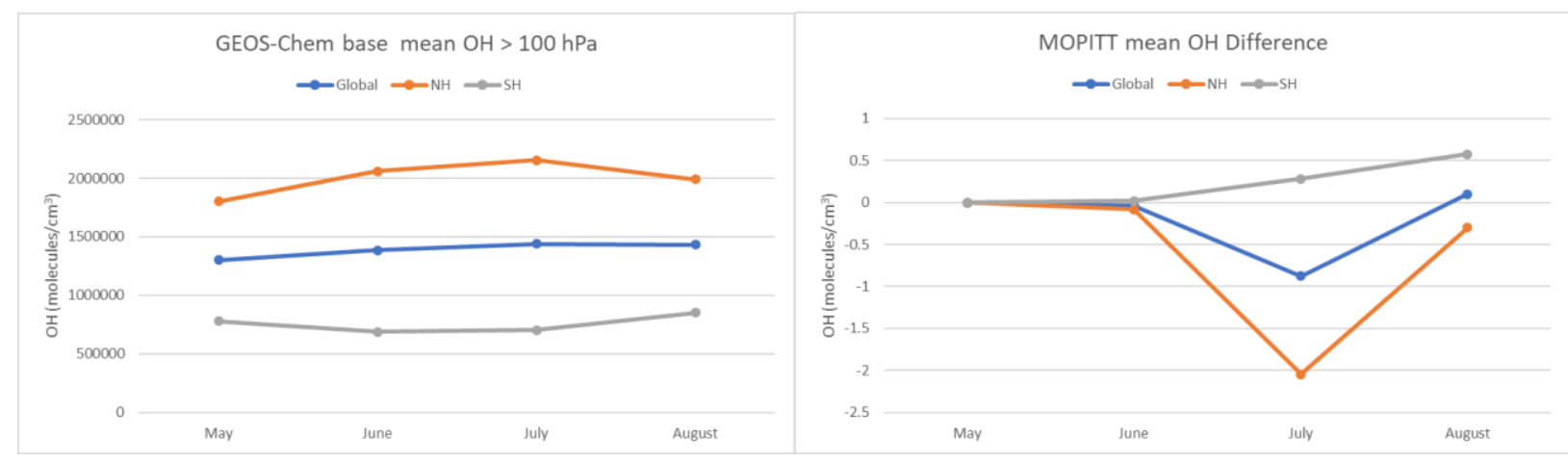

Figure 4.3.1.1: GEOS-Chem base modeled mean global OH for each month, May through August 2016. The left-hand plot shows the GEOS-Chem base model's global, $\mathrm{NH}$ and $\mathrm{SH}$ mean $\mathrm{OH}$ in molecules $/ \mathrm{cm}^{3}$. The right-hand plot shows the difference between the GEOS-Chem base and MOPITT assimilation mean $\mathrm{OH}$ difference in molecules $/ \mathrm{cm}^{3}$.

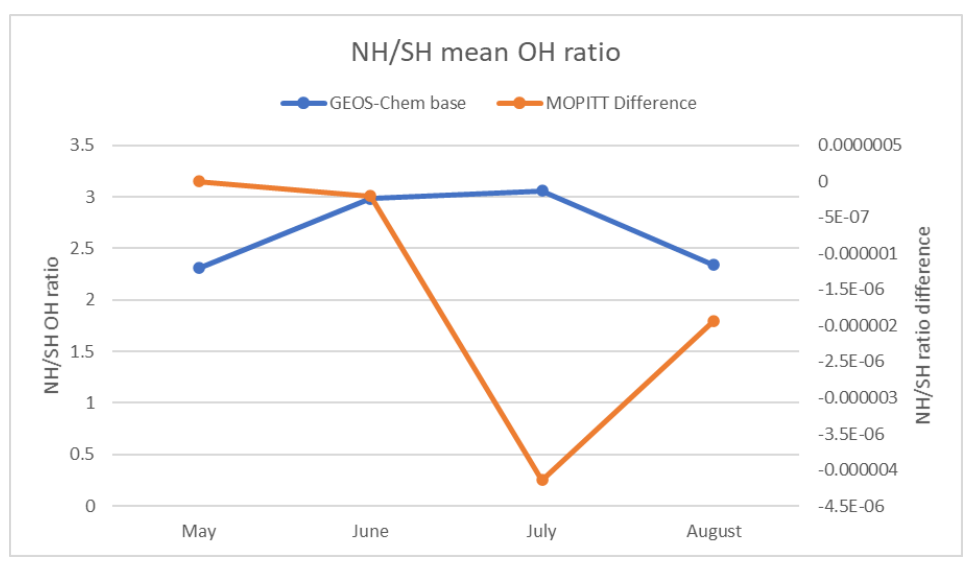

Figure 4.3.1.2: Mean NH/SH OH ratio each month, May through August 2016. Blue representing the GEOS-Chem base model ratio, and orange representing the ratio difference between the GEOS-Chem and MOPITT assimilation model. 
Figure 4.3.1.3 shows $\mathrm{OH}$ concentrations are consistently high for all four months over three specific areas: the Americas encompassing southern North America and Central America; Africa, specifically around the Sahel region; and Asia encompassing South Asia, East Asia, and part of Southeast Asia. The $\mathrm{OH}$ abundance appears to be smallest in May, limited to a thinner vertical distribution between $10^{\circ}$ and $30^{\circ}$ latitude. The $\mathrm{OH}$ abundance appears largest in July, where the vertical distribution widens to between $10^{\circ}$ and $50^{\circ}$ latitude with greater concentrations penetrating higher into the troposphere. The stronger concentrations also appear to have a smaller latitudinal spread within the boundary layer, then bulge towards the Southern Hemisphere just above the boundary layer. In June and July, slightly higher concentrations spread north in the upper troposphere. The MOPITT integrated model presents these same characteristics and are shown in Appendix B.6. 
i) May 2016 GEOS-Chem global OH

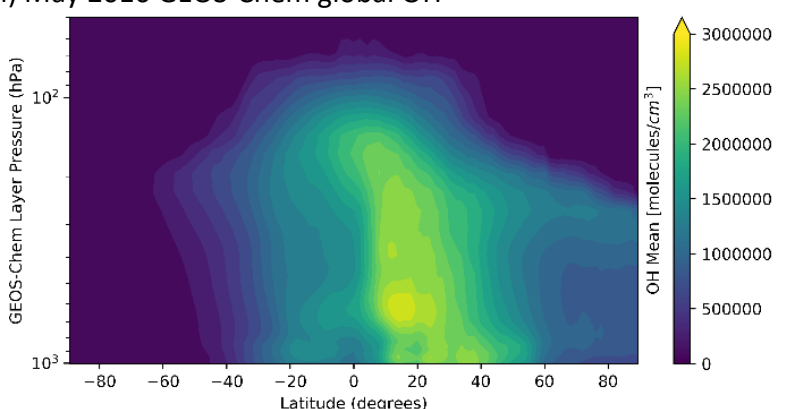

ii) June 2016 GEOS-Chem global OH

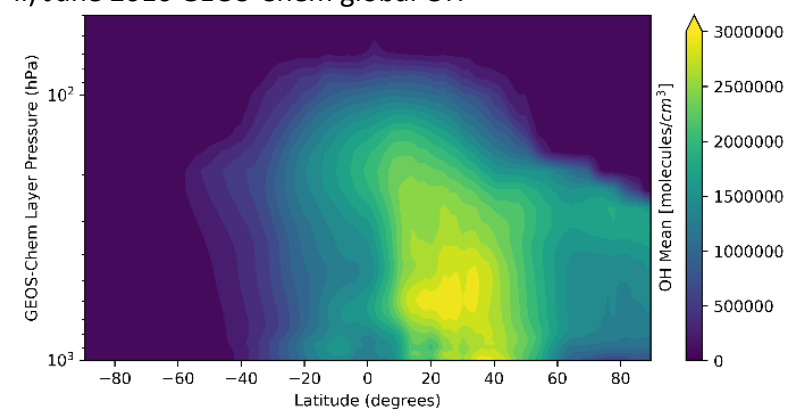

iii) July 2016 GEOS-Chem global OH



iv) August 2016 GEOS-Chem global OH

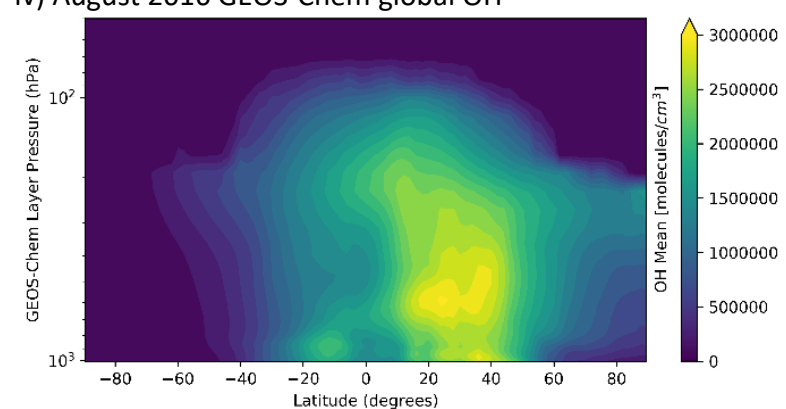

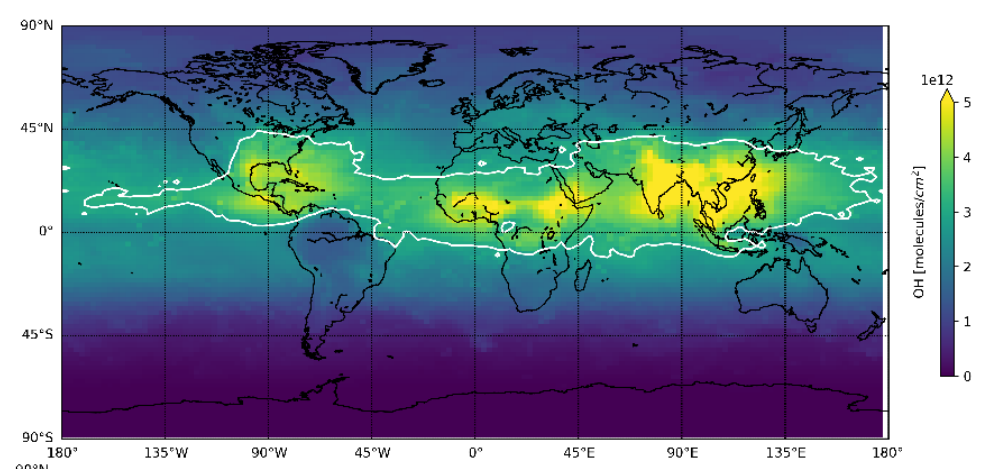
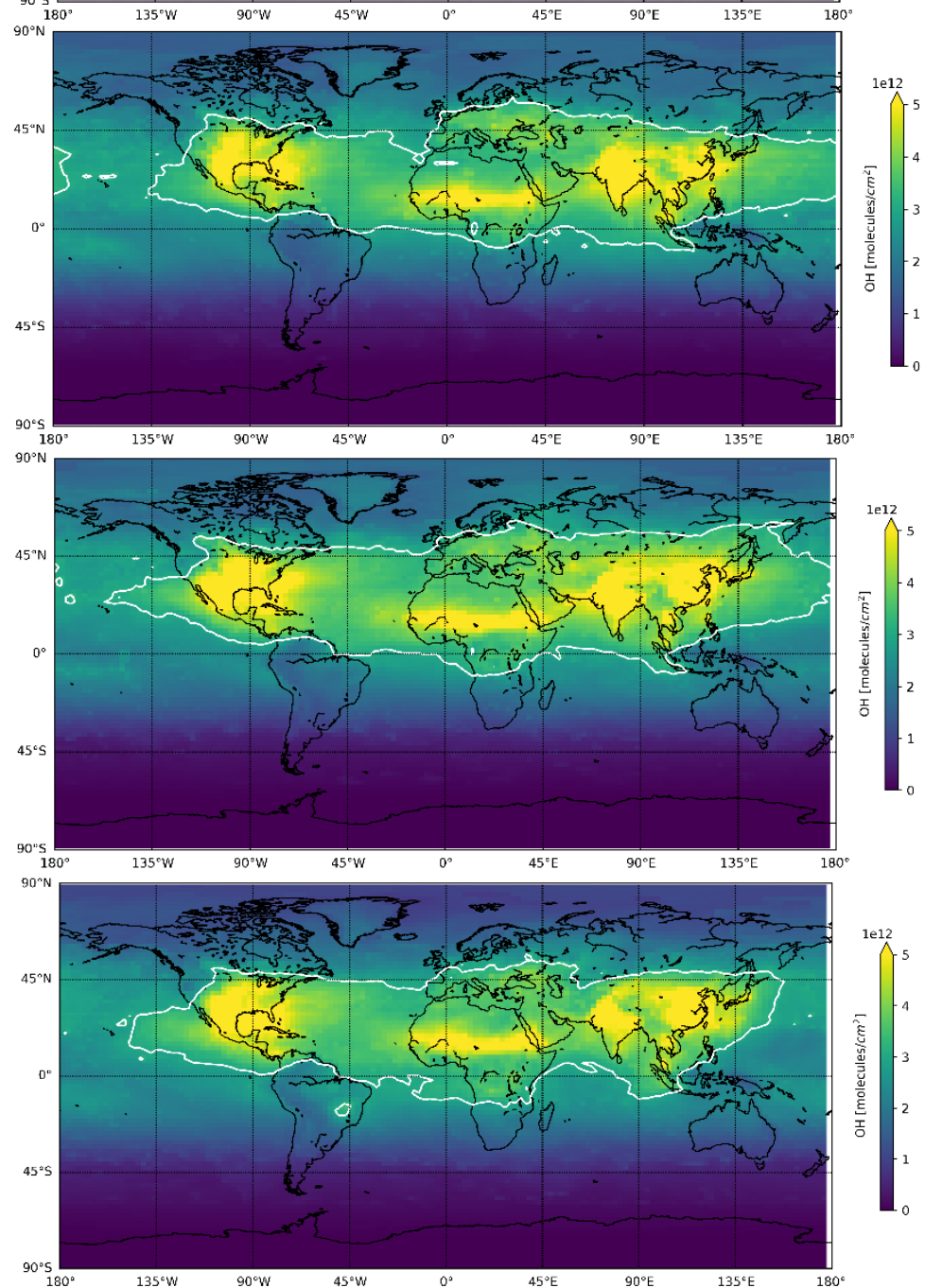

Figure 4.3.1.3: GEOS-Chem base modeled mean global OH distribution for each month, May through August 2016. Figures on the left represent the $\mathrm{OH}$ longitudinal molecules $/ \mathrm{cm}^{3}$ summation around the globe. Figures on the right represent to total column CO concentration in molecules $/ \mathrm{cm}^{2}$. The white contour line represents the MOPITT integrated model global $\mathrm{OH}$ concentration's $80^{\text {th }}$ percentile. 
Figure 4.3.1.4 shows the single iteration MOPITT assimilation $\mathrm{OH}$ distribution minus the GEOS-Chem base distribution. These $\mathrm{OH}$ distributions are observed to be similar to $\mathrm{CO}$ distributions in Section 4.2.1. Blue represents where the GEOS-Chem base and MOPITT model output the same $\mathrm{OH}$ concentrations. Purple represents where the GEOS-Chem base model outputs higher concentrations, and yellow represents where the MOPITT model outputs higher concentrations.

May shows scattered minor positive and negative differences, with a yellow band stretching across the mid-Pacific within the boundary layer. June shows similar upper troposphere differences spanning from Africa across over to Australasia. These differences grow in July and August both vertically and horizontally, although not to the same extent as the CO differences. Unlike the August CO distribution differences in Figure 4.2.1.2, the models appear to agree in Southern Hemisphere's the lower troposphere, and within the upper troposphere between $60^{\circ}$ and $90^{\circ}$ latitude. There also appears to be a band of scattered differences in the upper troposphere just north of Antarctica. 

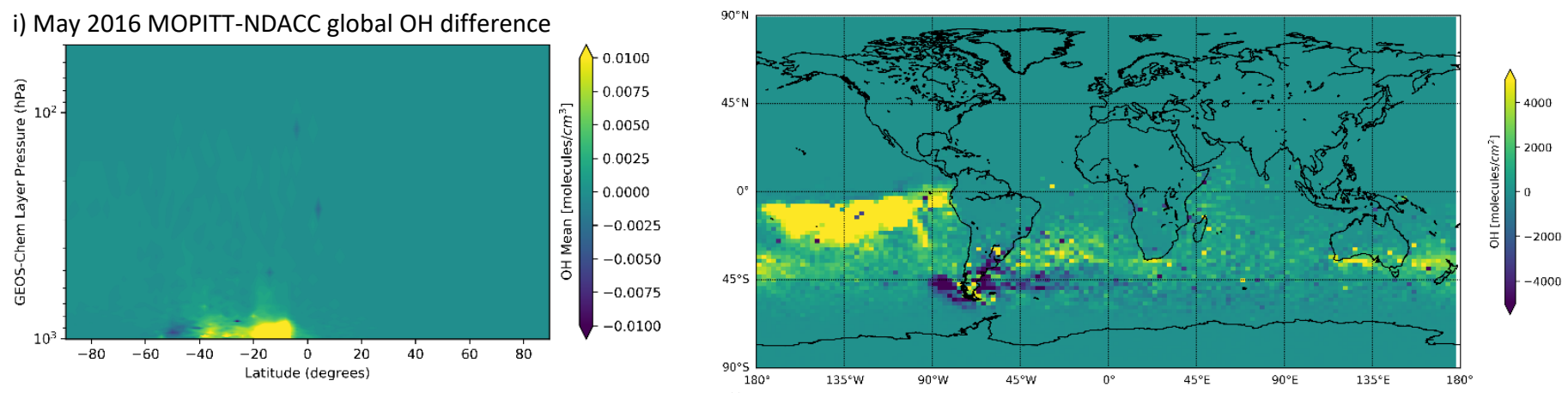

ii) June 2016 MOPITT-NDACC global OH difference
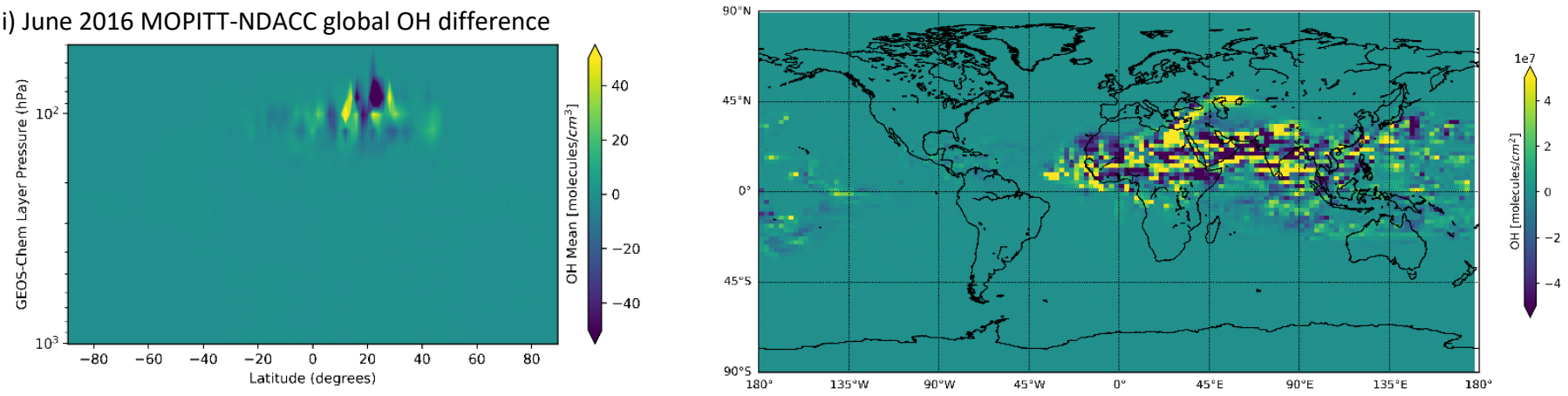

iii) July 2016 MOPITT-NDACC global OH difference
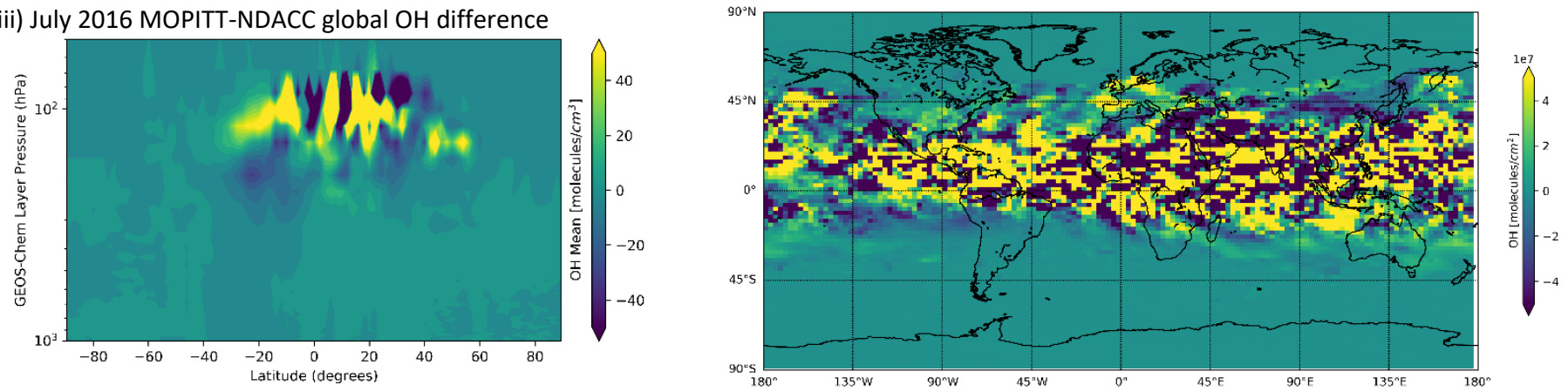

iv) August 2016 MOPITT-NDACC global OH difference
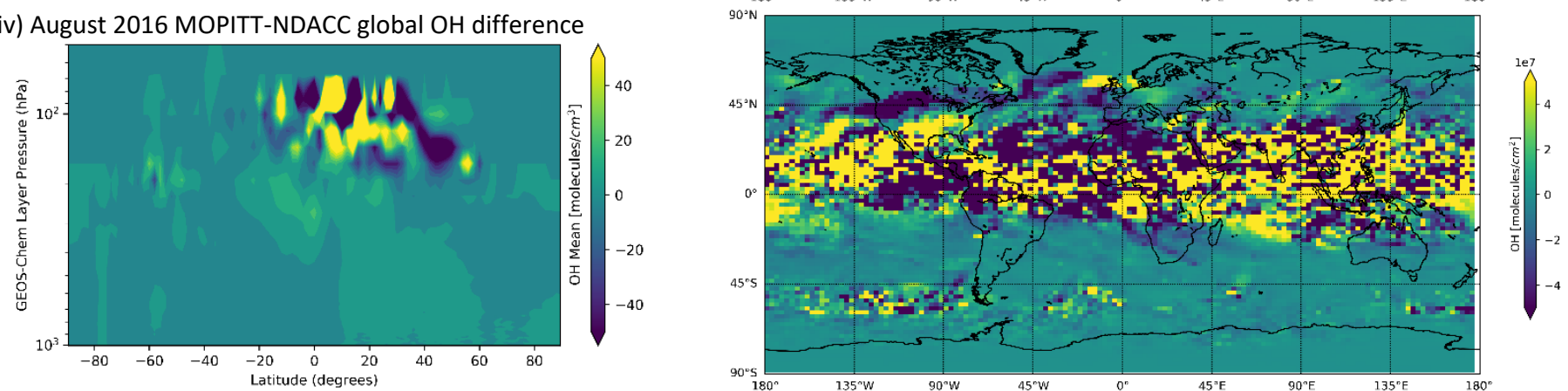

Figure 4.3.1.4: MOPITT minus the GEOS-Chem base global OH distribution and for each month, May through August 2016. Figures on the left represent the $\mathrm{OH}$ longitudinal molecules $/ \mathrm{cm}^{3}$ summation difference around the globe. Figures on the right represent to total column $\mathrm{OH}$ concentration difference in molecules $/ \mathrm{cm}^{2}$. Note that the MOPITT OH distribution used is the model output after one adjoint iteration. 


\subsubsection{Discussion}

The $[\mathrm{OH}]_{G M}$ and $\mathrm{OH}$ distributions between the GOES-Chem base and MOPITT integrated outputs are nearly indistinguishable from each other. Over the four months $[\mathrm{OH}]_{\mathrm{GM}}$ ranges between $13.02 \times 10^{5}$ to $14.38 \times 10^{5}$ molecules $/ \mathrm{cm}^{3}$ (Table 4.3.1.1). This is greater than all referenced literature in Table 2.3.1.1, including when the decadal average IAV is included from Turner et. al. (2018). This being noted, the $[\mathrm{OH}]_{\mathrm{GM}}$ of these references and the IAV from Turner are reflective of annual $[\mathrm{OH}]_{\mathrm{GM}}$ rather than Northern Hemisphere summer months. This study's 2016 time window also does not overlap with Table 2.3.1.1 literature except for Wolfe et.al. (2019). However, their domain is for $70 \%$ of tropospheric mass rather than below $100 \mathrm{hPa}$ reported in this study. This study's larger $[\mathrm{OH}]_{\mathrm{GM}}$ is not unforeseen as global $\mathrm{OH}$ is expected to be greater during the Northern Hemisphere's summer months due to insolation and declining northern CO loading.

May though August, strong $\mathrm{OH}$ concentrations are shown over three distinct regions in the Northern Hemisphere: southern North and Central America, the Sahel, and South to Eastern Asia (Figure 4.3.1.3). Looking at the $\mathrm{CO}$ and sensitivity distributions in Section 4.2, there are no obvious relationships found for these three regions, especially with minimal differences between the $\mathrm{OH}$ outputs. Further adjoint iterations in future studies may reveal noticeable relationships between regions as discussed in Section 4.2.

In May, Figure 4.3.1.4 shows scattered minor differences are found in the Southern Hemisphere like in Figure 4.2.1.2. Again, likely due to randomly generated numerical noise. Slightly larger $\mathrm{OH}$ concentrations are observed in the MOPITT assimilation output over the Pacific within the Southern Hemisphere's boundary layer. This is likely related to the same 
lower CO concentrations output by the MOPITT assimilation in Figure 4.2.1.2 over the same region. It is also noted that May has the greatest overall CO concentrations, and therefore show the lowest $[\mathrm{OH}]_{\mathrm{GM}}$ in May. These observations demonstrate the $\mathrm{CO}$ and $\mathrm{OH}$ relationship operating within the model.

From June to August, most $\mathrm{OH}$ differences lie in the upper troposphere like in the $\mathrm{CO}$ differences seen in Figure 4.2.1.2. Again, this is likely a result of propagated error generated from the model's variable tropopause. Interestingly, the $\mathrm{OH}$ distribution differences do not reflect the same spatial differences as the CO distribution comparison in July and August. The MOPITT $\mathrm{OH}$ does not show noticeable differences within the upper troposphere between $60^{\circ}$ and $90^{\circ}$ latitude, nor within the low to mid-troposphere in the Southern Hemisphere like the CO comparison (Figure 4.2.1.2). Here, the $\mathrm{CO}$ and $\mathrm{OH}$ relationship is not demonstrated. It is possible that the $\mathrm{CO}$ differences are not large enough in these regions to reflect differences in $\mathrm{OH}$. Alternatively, it could indicate a stronger relationship to an unknown factor influencing $\mathrm{OH}$ within these regions. Further MOPITT assimilation iterations may reveal more about these possible connections.

It should be noted that using satellite CO retrievals rather than in-situ retrievals to determine $\mathrm{OH}$ does have its limitations despite being able to provide greater vertical resolutions at an instance in time. The satellite retrievals used in this study are not geostationary in order to provide global coverage. However, this limits observations of a given area to the satellites overpass time. For example, with CrIS and TROPOMI having equatorial overpass times in the early afternoon, these instrument CO retrievals would be representative of the generally higher $\mathrm{CO}$ concentrations given in a day from anthropogenic activity. It is not 
representative of hourly trends throughout the day. Satellite retrievals are also more complex with varying sensitivity, a priori, and model assumptions embedded within the data product. All of which can effect the GEOS-Chem model assimilation sensitivities and $\mathrm{OH}$ outputs.

In conclusion, the single MOPITT CO assimilation iteration provides an initial look into its influence on the GEOS-Chem $\mathrm{OH}$ outputs. A seemingly propagating upper troposphere error due to a variable tropopause is observed in both Section 4.2 and 4.3. It is therefore recommended for future analysis to assimilate MOPITT (and other instruments) using a fixed tropopause. Minor differences in CO distribution between the MOPITT and GEOS-Chem base models in May are observed to impact $\mathrm{OH}$ distribution, but this relationship appears to be missing in later months. MOPITT's greater global sensitivities to $\mathrm{CO}$ emissions and the $\left[\mathrm{CH}_{4}+\mathrm{OH}\right]$ reaction rate has a significantly larger global coverage and has a clear initial impact on the model chemistry. However, no noticeable relationships are yet observed between sensitivities and their impact on $\mathrm{OH}$.

Further iterations may reveal more definite relationships between observations discussed Sections 4.2 and 4.3. It may also be beneficial to conduct experimental model runs varying the regularisation parameter to further minimise (therefore optimise) the cost function. Relationships to look out for include ties to the three regions of high $\mathrm{OH}$; missing links between $\mathrm{CO}$ and $\mathrm{OH}$ concentrations within the Southern Hemisphere lower troposphere and for the $60^{\circ}$ and $90^{\circ}$ latitude upper troposphere; and the apparent $\left[\mathrm{CH}_{4}+\mathrm{OH}\right]$ reaction rate sensitivity separations along the $110 \mathrm{hPa}$ level. Multiple iteration runs for the MOPITT assimilation was attempted for this study, but the model would produce negative cost functions which 
eventually led to crashes and illogical outputs. Investigation into the cause is required, but it is believed to be related to the variable tropopause and a MOPITT assimilation input error.

Once a multiple iteration MOPITT assimilation and analysis is successful, it is recommended that an assimilation with CrIS and TROPOMI CO observations be conducted. $\mathrm{A}$ CrIS and TROPOMI assimilation were not included in this study as additional model development is required. These instruments have the potential to define finer spatial and temporal $\mathrm{OH}$ resolution within the atmosphere. $\mathrm{CrIS}^{\prime}$ s greater $\mathrm{CO}$ sensitivity in the free troposphere may reveal more information on $\mathrm{OH}$ distribution and chemistry. TROPOMI's greater total column resolution may better constrain $\mathrm{CO}$ emissions, and therefore has potential to better constrain $\mathrm{OH}$ distributions towards the surface. Once the Joint CrIS-TROPOMI product is available, together they have the potential to provide superior global $\mathrm{OH}$ distribution information within the GEOS-Chem model than MOPITT. 


\subsection{Conclusion}

In past decades, global $\mathrm{OH}$ has been inversely calculated from the trace gas MCF. Since the implementation of the Montreal Protocol, global MCF concentrations have greatly declined and is therefore less reliable to infer $\mathrm{OH}$ for the future (Montzka et al., 2011). In recent years, research into alternative trace gases to infer global $\mathrm{OH}$ has expanded. This study investigates the use of $\mathrm{CO}$ as a trace gas by assessing new and existing $\mathrm{CO}$ observation platforms, their sensitivities, and their impact on global OH in the GEOS-Chem adjoint model.

\subsection{Summary of results}

Of the instruments assessed, this investigation found that MOPITT currently offers the most accurate representation of the $\mathrm{CO}$ total columns and profiles in comparison to NDACC. The mature MOPITT NIR-TIR product offers fair sensitivity throughout its tropospheric vertical profile. CrIS profiles have greater resolution than MOPITT, but currently only offer greater accuracy against NDACC at elevations above $700 \mathrm{hPa}$ where sensitivity is best (Smith et al., 2020b). Below $700 \mathrm{hPa}$, the CrIS CLIMCAPS algorithm tends to overestimate concentration and more heavily rely on the a priori. Initial comparisons of TROPOMI CO total columns show close agreement with MOPITT and offer a promising quality resolution. Although without a proper cross-kernel comparison and more information on the TROPOMI averaging kernels, this analysis holds limited value.

The GEOS-Chem adjoint sensitivity analysis of the NDACC network locations and the MOPITT CO assimilation accentuate the importance of utilising global coverage satellite datasets to infer $\mathrm{OH}$. The MOPITT sensitivity analysis demonstrates the greater horizontal 
coverage over the spatially constrained NDACC network, having a more significant impact on $\mathrm{OH}$ within the GEOS-Chem model. In addition, MOPITT assimilation figures show arbitrary sensitivity division lines vertically at the equator and horizontally at approximately $110 \mathrm{hPa}$. Without further adjoint iterations it is undetermined if this is the product of model bias, incomplete assimilation, or a characteristic of $\mathrm{OH}$ chemistry.

The $\mathrm{CO}$ and therefore $\mathrm{OH}$ abundance and distribution did not change much between a single iteration assimilation of MOPITT observations and the GEOS-Chem base. Modeled $[\mathrm{OH}]_{\mathrm{GM}}$ and $\mathrm{NH} / \mathrm{SH}$ interhemispheric ratios were high compared to past literature but are within reason considering seasonal and interannual variability trends. Not many immediately obvious unique spatial or temporal relationships were found between $\mathrm{CO}, \mathrm{OH}$, and sensitivities other than lower $\mathrm{CO}$ inferring greater $\mathrm{OH}$. However, initial analysis did reveal elements to pay attention to in future analysis such as the mentioned sensitivity division lines and areas where the expected $\mathrm{CO}$ to $\mathrm{OH}$ relationship does not appear to exist.

In conclusion, $\mathrm{CO}$ is still a tracer candidate to replace MCF in order to determine global $\mathrm{OH}$ so that we may continue to assess the oxidative capacity of the atmosphere. In addition, CO's shorter lifetime in combination with greater satellite resolution has potential to reveal regional $\mathrm{OH}$ trends. The high resolution $\mathrm{CrIS}$ and TROPOMI products, and eventually the Joint CrIS-TROPOMI product, offer the opportunity to analyse these trends and therefore improve our understanding of regional air quality chemistry. 


\section{$5.2 \quad$ Future recommendations}

Now that most 2019 NDACC station data is publicly available, it is recommended that this CO intercomparison study be repeated with 2019 data across all observing platforms. This would allow for true temporal evaluations with available CrIS and TROPOMI data. It is also recommended to evaluate $\mathrm{CO}$ over an entire year rather than four months. A year would allow for observations of possible seasonal trends and should also allow for more coinciding data between instruments for comparison. In addition, it is advised that further GEOS-Chem adjoint iterations be conducted to minimise the cost function and optimise MOPITT CO assimilation to reveal true differences within the model. This should be modeled with a fixed tropopause as analysis revealed likely propagating error from employing a variable tropopause.

Although CrIS and TROPOMI's respective CO profiles and total columns do not currently offer a well-rounded tropospheric data product for inferring OH like MOPITT, it is still proposed to assimilate both data sets into GEOS-Chem for analysis in future research. The significantly greater spatial and temporal resolution of the CLIMCAPS dataset may offer greater insight into spatial $\mathrm{CO}$ and therefore $\mathrm{OH}$ trends within the troposphere at elevations greater than $700 \mathrm{hPa}$. The better total column resolution and implied better sensitivity to the surface of TROPOMI may also offer greater insight into surface and boundary $\mathrm{OH}$. These individual instrument comparisons may then be used for comparison against a future assimilation of the Joint CrISTROPOMI product. The joint product is expected to offer superior CO accuracy, precision, and resolution and therefore $\mathrm{OH}$ within the GEOS-Chem model than MOPITT. This improved observational bound on $\mathrm{OH}$ abundance would enhance understanding of chemical reaction rates in the atmosphere and improve global atmospheric model calculations. 


\subsection{References}

Adjoint Model and Data Assimilation Working Group. (2020, March 18). Geos-chem Adjoint Wiki. Geos-Chem Wiki. http://wiki.seas.harvard.edu/geos-chem/index.php/GEOSChem_Adjoint

Amedro, D., Miyazaki, K., Parker, A., Schoemaecker, C., \& Fittschen, C. (2012). Atmospheric and kinetic studies of $\mathrm{OH}$ and $\mathrm{HO} 2$ by the FAGE technique. Journal of Environmental Sciences, 24(1), 78-86. https://doi.org/10.1016/S1001-0742(11)60723-7

Babić, L., Kissi-Ameyaw, J., Loots, E., Smeets, J., Braak, R., Kleipool, Q., Ludewig, A., Vacanti, G., Dierssen, W., Leloux, J., \& Rozemeijer, N. (2017). Algorithm theoretical basis document for the TROPOMI L01b data processor (ATBD No. S5P-KNMI-L01B-0009-SD). Royal Netherlands Meteorological Institute KNMI. https://sentinels.copernicus.eu/documents/247904/2476257/Sentinel-5P-TROPOMILevel-1B-ATBD

Barnet, C. (2019). Sounder SIPS: JPSS-1 CrIS Level 2 CLIMCAPS: Atmosphere cloud and surface geophysical state V2 (Version 2) [Computer software]. Goddard Earth Sciences Data and Information Services Center (GES DISC). https://doi.org/10.5067/LESQUBLWS18H

Batchelor, R. L., Strong, K., Lindenmaier, R., Mittermeier, R. L., Fast, H., Drummond, J. R., \& Fogal, P. F. (2009). A New Bruker IFS 125HR FTIR Spectrometer for the Polar Environment Atmospheric Research Laboratory at Eureka, Nunavut, Canada: Measurements and Comparison with the Existing Bomem DA8 Spectrometer. Journal of Atmospheric and Oceanic Technology, 26(7), 1328-1340.

https://doi.org/10.1175/2009JTECHA1215.1 
Berrick, S. (2020). GES DISC Dataset: Sounder SIPS: JPSS-1 CrIS Level 2 CLIMCAPS: Atmosphere cloud and surface geophysical state V2 (SNDRJ1IML2CCPRET 2). EARTHDATA. https://disc.gsfc.nasa.gov/api/jobs/results/5ecfd3591fb4c4829b4b0d67

Bey, I., Jacob, D. J., Yantosca, R. M., Logan, J. A., Field, B. D., Fiore, A. M., Li, Q., Liu, H. Y., Mickley, L. J., \& Schultz, M. G. (2001). Global modeling of tropospheric chemistry with assimilated meteorology: Model description and evaluation. Journal of Geophysical Research: Atmospheres, 106(D19), 23073-23095.

https://doi.org/10.1029/2001JD000807

Birner, T. (2010). Residual Circulation and Tropopause Structure. Journal of the Atmospheric Sciences, 67(8), 2582-2600. https://doi.org/10.1175/2010JAS3287.1

Bohren, C. F., \& Albrecht, B. A. (1998). Atmospheric Thermodynamics. Oxford University Press. Borsdorff, T., Aan de Brugh, J., Hu, H., Aben, I., Hasekamp, O., \& Landgraf, J. (2018). Measuring Carbon Monoxide With TROPOMI: First Results and a Comparison With ECMWF-IFS Analysis Data. Geophysical Research Letters, 45(6), 2826-2832. https://doi.org/10.1002/2018GL077045

Borsdorff, T., Hasekamp, O. P., Wassmann, A., \& Landgraf, J. (2014). Insights into Tikhonov regularization: Application to trace gas column retrieval and the efficient calculation of total column averaging kernels. Atmospheric Measurement Techniques, 7(2), 523-535. https://doi.org/10.5194/amt-7-523-2014

Bousquet, P., Hauglustaine, D. A., Peylin, P., Carouge, C., \& Ciais, P. (2005). Two decades of OH variability as inferred by an inversion of atmospheric transport and chemistry of methyl 
chloroform. Atmospheric Chemistry and Physics, 5(10), 2635-2656.

https://doi.org/10.5194/acp-5-2635-2005

Brenninkmeijer, C. A. M., Manning, M. R., Lowe, D. C., Wallace, G., Sparks, R. J., \& Volz-Thomas, A. (1992). Interhemispheric asymmetry in $\mathrm{OH}$ abundance inferred from measurements of atmospheric 14CO. Nature, 356(6364), 50-52. https://doi.org/10.1038/356050a0

Buchholz, R. R., Deeter, M. N., Worden, H. M., Gille, J., Edwards, D. P., Hannigan, J. W., Jones, N. B., Paton-Walsh, C., Griffith, D. W. T., Smale, D., Robinson, J., Strong, K., Conway, S., Sussmann, R., Hase, F., Blumenstock, T., Mahieu, E., \& Langerock, B. (2017). Validation of MOPITT carbon monoxide using ground-based Fourier transform infrared spectrometer data from NDACC. Atmospheric Measurement Techniques, 10(5), 19271956. https://doi.org/10.5194/amt-10-1927-2017

Butchart, N. (2014). The Brewer-Dobson circulation. Reviews of Geophysics, 52(2), 157-184. https://doi.org/10.1002/2013RG000448

Cao, C., Chen, Y., Jin, X., Tremblay, D., Wald, A., Kireev, S., Yu, H., \& Wang, L. (2018). Cross Track Infrared Sounder (CrIS) Sensor Data Record (SDR) User's Guide Version 1.1. U.S. DEPARTMENT OF COMMERCE; National Oceanic and Atmospheric Administration; National Environmental Satellite, Data, and Information Service. https://www.star.nesdis.noaa.gov/jpss/documents/UserGuides/CrIS_SDR_Users_Guide 1p1_20180405.pdf

Center for International Earth Science Information \& Network(CIESIN)/Columbia University. (2021). National Aggregates of Geospatial Data Collection (NAGDC) / SEDAC. NASA 
Socioeconomic Data and Applications Center (SEDAC).

https://sedac.ciesin.columbia.edu/data/collection/nagdc

Copernicus Sentinel-5P (processed by ESA). (2018). TROPOMI Level 2 Carbon Monoxide total column products (Version 1) [Data set]. European Space Agency. https://doi.org/10.5270/s5p-1hkp7rp

Dalsøren, S. B., Myhre, C. L., Myhre, G., Gomez-Pelaez, A. J., Søvde, O. A., Isaksen, I. S. A., Weiss, R. F., \& Harth, C. M. (2016). Atmospheric methane evolution the last 40 years. Atmospheric Chemistry and Physics, 16(5), 3099-3126. https://doi.org/10.5194/acp-163099-2016

De Mazière, M., Thompson, A. M., Kurylo, M. J., Wild, J. D., Bernhard, G., Blumenstock, T., Braathen, G. O., Hannigan, J. W., Lambert, J.-C., Leblanc, T., McGee, T. J., Nedoluha, G., Petropavlovskikh, I., Seckmeyer, G., Simon, P. C., Steinbrecht, W., \& Strahan, S. E. (2018). The Network for the Detection of Atmospheric Composition Change (NDACC): History, status and perspectives. Atmospheric Chemistry and Physics, 18(7), 4935-4964. https://doi.org/10.5194/acp-18-4935-2018

Deeter, M. N., Edwards, D. P., Francis, G. L., Gille, J. C., Martínez-Alonso, S., Worden, H. M., \& Sweeney, C. (2017). A climate-scale satellite record for carbon monoxide: The MOPITT Version 7 product. Atmospheric Measurement Techniques, 10(7), 2533-2555. https://doi.org/10.5194/amt-10-2533-2017

Deeter, M. N., Edwards, D. P., Gille, J. C., \& Worden, H. M. (2015). Information content of MOPITT CO profile retrievals: Temporal and geographical variability: MOPITT 
INFORMATION CONTENT VARIABILITY. Journal of Geophysical Research: Atmospheres, 120(24), 12723-12738. https://doi.org/10.1002/2015JD024024

Deeter, M. N., Martínez-Alonso, S., Edwards, D. P., Emmons, L. K., Gille, J. C., Worden, H. M., Pittman, J. V., Daube, B. C., \& Wofsy, S. C. (2013). Validation of MOPITT Version 5 thermal-infrared, near-infrared, and multispectral carbon monoxide profile retrievals for 2000-2011: VALIDATION OF MOPITT VERSION 5 RETRIEVALS. Journal of Geophysical Research: Atmospheres, 118(12), 6710-6725. https://doi.org/10.1002/jgrd.50272

Deeter, M. N., MOPITT Algorithm Development Team, Atmospheric Chemistry Observations and Modeling Laboratory, \& National Center for Atmospheric Research. (2017). MOPITT (Measurements of Pollution in the Troposphere) Version 7 Product User's Guide. https://www2.acom.ucar.edu/sites/default/files/mopitt/v7_users_guide_201707.pdf

Dentener, F., Peters, W., Krol, M. C., van Weele, M., Bergamaschi, P., \& Lelieveld, J. (2003). Interannual variability and trend of $\mathrm{CH} 4$ lifetime as a measure for $\mathrm{OH}$ changes in the 1979-1993 time period. Journal of Geophysical Research, 108(D15), 4442. https://doi.org/10.1029/2002JD002916

Drummond, J. R., Zou, J., Nichitiu, F., Kar, J., Deschambaut, R., \& Hackett, J. (2010). A review of 9-year performance and operation of the MOPITT instrument. Advances in Space Research, 45(6), 760-774. https://doi.org/10.1016/j.asr.2009.11.019

Encyclopedia Britannica. (2010). Atmospheric circulation / meteorology. Encyclopedia Britannica. https://www.britannica.com/science/atmospheric-circulation 
Errico, R. M. (1997). What Is an Adjoint Model? Bulletin of the American Meteorological Society, 78(11), 2577-2592. https://doi.org/10.1175/15200477(1997)078<2577:WIAAM>2.0.CO;2

Fittschen, C., Al Ajami, M., Batut, S., Ferracci, V., Archer-Nicholls, S., Archibald, A. T., \& Schoemaecker, C. (2019). ROOOH: A missing piece of the puzzle for $\mathrm{OH}$ measurements in low-NO environments? Atmospheric Chemistry and Physics, 19(1), 349-362. https://doi.org/10.5194/acp-19-349-2019

Francis, G. L., Deeter, M. N., Martinez Alonso, S., Gille, J. C., Edwards, D. P., Mao, D., Worden, H. M., \& Ziskin, D. (2017). MOPITT Measurement of Pollution in the Troposphere Algorithm Theoretical Basis Document: Retrieval of Carbon Monoxide Profiles and Column Amounts from MOPITT Observed Radiances (Level 1 to Level 2). Atmospheric Chemistry Observations and Modeling Laboratory National Center for Atmospheric Research.

Fu, D., Bowman, K. W., Worden, H. M., Natraj, V., Worden, J. R., Yu, S., Veefkind, P., Aben, I., Landgraf, J., Strow, L., \& Han, Y. (2016). High-resolution tropospheric carbon monoxide profiles retrieved from CrIS and TROPOMI. Atmospheric Measurement Techniques, 9(6), 2567-2579. https://doi.org/10.5194/amt-9-2567-2016

Fu, Q., Johanson, C. M., Wallace, J. M., \& Reichler, T. (2006). Enhanced Mid-Latitude Tropospheric Warming in Satellite Measurements. Science, 312(5777), 1179-1179. https://doi.org/10.1126/science.1125566

Gambacorta, A. (2013). The NOAA Unique CrIS/ATMS Processing System (NUCAPS): Algorithm Theoretical Basis Documentation (1.0) [ATBD]. NOAA Center for Weather and Climate Prediction. 
https://www.ospo.noaa.gov/Products/atmosphere/soundings/nucaps/docs/NUCAPS_A TBD_20130821.pdf

Gaubert, B., Arellano, A. F., Barré, J., Worden, H. M., Emmons, L. K., Tilmes, S., Buchholz, R. R., Vitt, F., Raeder, K., Collins, N., Anderson, J. L., Wiedinmyer, C., Martinez Alonso, S., Edwards, D. P., Andreae, M. O., Hannigan, J. W., Petri, C., Strong, K., \& Jones, N. (2016). Toward a chemical reanalysis in a coupled chemistry-climate model: An evaluation of MOPITT CO assimilation and its impact on tropospheric composition: CHEMICAL RESPONSE FROM CO ASSIMILATION. Journal of Geophysical Research: Atmospheres, 121(12), 7310-7343. https://doi.org/10.1002/2016JD024863

Gaubert, B., Worden, H. M., Arellano, A. F. J., Emmons, L. K., Tilmes, S., Barré, J., Martinez Alonso, S., Vitt, F., Anderson, J. L., Alkemade, F., Houweling, S., \& Edwards, D. P. (2017). Chemical Feedback From Decreasing Carbon Monoxide Emissions. Geophysical Research Letters, 44(19), 9985-9995. https://doi.org/10.1002/2017GL074987

GEOS-Chem Support Team. (2021). GEOS-Chem. GEOS-Chem. http://www.geos-chem.org GEOS-Chem Support Team, GEOS-Chem Working Groups, GEOS-Chem Steering Committee, \& GEOS-Chem user community. (2021). Geos-chem Wiki. Geos-Chem Wiki. http://wiki.seas.harvard.edu/geos-chem/index.php/Main_Page Gille, J., \& NCAR MOPITT Team. (1996). MOPITT Measurement of Pollution in the Troposphere Algorithm Theoretical Basis Document: Retrieval of Carbon Monoxide Profiles and Column Amounts of Cabon Monoxide and Methane from Observed Radiances (Level 1 to Level 2) Version 3 DRAFT. https://eospso.nasa.gov/sites/default/files/atbd/atbd-mop02.pdf 
Hakami, A., Henze, D. K., Seinfeld, J. H., Chai, T., Tang, Y., Carmichael, G. R., \& Sandu, A. (2005). Adjoint inverse modeling of black carbon during the Asian Pacific Regional Aerosol Characterization Experiment: ADJOINT INVERSE MODELING OF BLACK CARBON. Journal of Geophysical Research: Atmospheres, 110(D14), n/a-n/a. https://doi.org/10.1029/2004JD005671

Haqq-Misra, J., Lee, S., \& Frierson, D. M. W. (2011). Tropopause Structure and the Role of Eddies. Journal of the Atmospheric Sciences, 68(12), 2930-2944. https://doi.org/10.1175/JAS-D-11-087.1

Hard, T. M., O’Brien, R. J., Chan, C. Y., \& Mehrabzadeh, A. A. (1984). Tropospheric free radical determination by fluorescence assay with gas expansion. Environmental Science \& Technology, 18(10), 768-777. https://doi.org/10.1021/es00128a009

Hase, F., Hannigan, J. W., Coffey, M. T., Goldman, A., Höpfner, M., Jones, N. B., Rinsland, C. P., \& Wood, S. W. (2004). Intercomparison of retrieval codes used for the analysis of highresolution, ground-based FTIR measurements. Journal of Quantitative Spectroscopy and Radiative Transfer, 87(1), 25-52. https://doi.org/10.1016/j.jqsrt.2003.12.008

Heard, D. E., \& Pilling, M. J. (2003). Measurement of $\mathrm{OH}$ and $\mathrm{HO} 2$ in the Troposphere. Chemical Reviews, 103(12), 5163-5198. https://doi.org/10.1021/cr020522s

Henze, D. K., Hakami, A., \& Seinfeld, J. H. (2007). Development of the adjoint of GEOS-Chem. Atmospheric Chemistry and Physics, 7(9), 2413-2433. https://doi.org/10.5194/acp-72413-2007

Holmes, C. D., Prather, M. J., Søvde, O. A., \& Myhre, G. (2013). Future methane, hydroxyl, and their uncertainties: Key climate and emission parameters for future predictions. 
Atmospheric Chemistry and Physics, 13(1), 285-302. https://doi.org/10.5194/acp-13$285-2013$

Hu, S., \& Vallis, G. K. (2019). Meridional structure and future changes of tropopause height and temperature. Quarterly Journal of the Royal Meteorological Society, 145(723), 26982717. https://doi.org/10.1002/qj.3587

Hu, Y., \& Fu, Q. (2007). Observed poleward expansion of the Hadley circulation since 1979. Atmospheric Chemistry and Physics, 7(19), 5229-5236. https://doi.org/10.5194/acp-7$5229-2007$

Hudson, R. D., Andrade, M. F., Follette, M. B., \& Frolov, A. D. (2006). The total ozone field separated into meteorological regimes - Part II: Northern Hemisphere mid-latitude total ozone trends. Atmospheric Chemistry and Physics, 6(12), 5183-5191. https://doi.org/10.5194/acp-6-5183-2006

Jacob, D. J. (2000). Introduction to Atmospheric Chemistry. Princeton University Press. https://press.princeton.edu/books/hardcover/9780691001852/introduction-toatmospheric-chemistry

Jöckel, P. (2001). Cosmogenic 14 CO as tracer for atmospheric chemistry and transport [Ph.D. Thesis, Heidelberg University]. http://doi.org/10.11588/heidok.00001426

Jones, D. B. A. (2019, December 9). Constraints on the oxidative capacity of the atmosphere: Implications of top-down CO emission estimates (abstract \#GC11K-1113) [Conference Poster]. American Geophysical Union Fall Meeting 2019, San Francisco, CA, United States. https://agu.confex.com/agu/fm19/meetingapp.cgi/Paper/626783 
JPSS. (n.d.). Mission and Instruments. Joint Polar Satellite System. Retrieved February 11, 2020, from https://www.jpss.noaa.gov/mission_and_instruments.html

Kerzenmacher, T., Dils, B., Kumps, N., Blumenstock, T., Clerbaux, C., Coheur, P.-F., Demoulin, P., García, O., George, M., Griffith, D. W. T., Hase, F., Hadji-Lazaro, J., Hurtmans, D., Jones, N., Mahieu, E., Notholt, J., Paton-Walsh, C., Raffalski, U., Ridder, T., ... De Mazière, M. (2012). Validation of IASI FORLI carbon monoxide retrievals using FTIR data from NDACC. Atmospheric Measurement Techniques, 5(11), 2751-2761. https://doi.org/10.5194/amt$5-2751-2012$

KNMI R\&D Satellite Observations. (n.d.). Mission Status. Tropomi. Retrieved February 12, 2020, from http://www.tropomi.eu/mission-status

Krol, M. C., \& Lelieveld, J. (2003). Can the variability in tropospheric $\mathrm{OH}$ be deduced from measurements of 1,1,1-trichloroethane (methyl chloroform)?: OH FROM METHYL CHLOROFORM MEASUREMENTS. Journal of Geophysical Research: Atmospheres, 108(D3), n/a-n/a. https://doi.org/10.1029/2002JD002423

Krol, M. C., Meirink, J. F., Bergamaschi, P., Mak, J. E., Lowe, D., Jöckel, P., Houweling, S., \& Röckmann, T. (2008). What can $14 \mathrm{CO}$ measurements tell us about OH? Atmospheric Chemistry and Physics, 8(16), 5033-5044. https://doi.org/10.5194/acp-8-5033-2008 Landgraf, J., ann de Brugh, J., Scheepmaker, R. A., Borsdorff, T., Houweling, S., \& Hasekamp, O. P. (2018). Algorithm Theoretical Baseline Document for Sentinel-5 Precursor: Carbon Monoxide Total Column Retrieval (ATBD SRON-S5P-LEV2-RP-002; Issue 1.10). SRON Netherlands Institute for Space Research. 
http://www.tropomi.eu/sites/default/files/files/SRON_S5P_LEV2_RP_002_issue1.10_C

O_signed.pdf

Langerock, B., Sha, M. K., Lambert, J.-C., Landgraf, J., \& Borsdorff, T. (2018). S5P MPC VDAF

Validation Web Article: Total Carbon Monoxide (No. S5P-MPC-VDAF-WVA-

L2_CO_20180716). European Space Agency.

Langford, A. O., Brioude, J., Cooper, O. R., Senff, C. J., Alvarez, R. J., Hardesty, R. M., Johnson, B.

J., \& Oltmans, S. J. (2012). Stratospheric influence on surface ozone in the Los Angeles area during late spring and early summer of 2010: STRATOSPHERIC INFLUENCE ON

SURFACE OZONE. Journal of Geophysical Research: Atmospheres, 117(D21), n/a-n/a. https://doi.org/10.1029/2011JD016766

Langford, A. O., Pierce, R. B., \& Schultz, P. J. (2015). Stratospheric intrusions, the Santa Ana winds, and wildland fires in Southern California: Stratospheric Intrusions and Wildfires. Geophysical Research Letters, 42(14), 6091-6097. https://doi.org/10.1002/2015GL064964

Langford, A. O., Senff, C. J., Alvarez, R. J., Brioude, J., Cooper, O. R., Holloway, J. S., Lin, M. Y., Marchbanks, R. D., Pierce, R. B., Sandberg, S. P., Weickmann, A. M., \& Williams, E. J. (2015). An overview of the 2013 Las Vegas Ozone Study (LVOS): Impact of stratospheric intrusions and long-range transport on surface air quality. Atmospheric Environment, 109, 305-322. https://doi.org/10.1016/j.atmosenv.2014.08.040

Lawrence, M. G., Jöckel, P., \& von Kuhlmann, R. (2001). What does the global mean OH concentration tell us? Atmospheric Chemistry and Physics, 1(1), 37-49. https://doi.org/10.5194/acp-1-37-2001 
Lelieveld, J., Brenninkmeijer, C., Joeckel, P., Isaksen, I., Krol, M. C., Mak, J., Dlugokencky, E., Montzka, S., Novelli, P., \& Peters, W. (2006). New Directions: Watching over tropospheric hydroxyl (OH). Atmospheric Environment, 40(29), 5741-5743. https://doi.org/10.1016/j.atmosenv.2006.04.008

Lelieveld, J., Dentener, F. J., Peters, W., \& Krol, M. C. (2004). On the role of hydroxyl radicals in the self-cleansing capacity of the troposphere. Atmospheric Chemistry and Physics, 4(9/10), 2337-2344. https://doi.org/10.5194/acp-4-2337-2004

Lelieveld, J., Gromov, S., Pozzer, A., \& Taraborrelli, D. (2016). Global tropospheric hydroxyl distribution, budget and reactivity. Atmospheric Chemistry and Physics, 16(19), 1247712493. https://doi.org/10.5194/acp-16-12477-2016

Lelieveld, J., Peters, W., Dentener, F. J., \& Krol, M. C. (2002). Stability of tropospheric hydroxyl chemistry. Journal of Geophysical Research: Atmospheres, 107(D23), ACH 17-1-ACH 1711. https://doi.org/10.1029/2002JD002272

Li, F., Waugh, D. W., Douglass, A. R., Newman, P. A., Strahan, S. E., Ma, J., Nielsen, J. E., \& Liang, Q. (2012). Long-term changes in stratospheric age spectra in the 21st century in the Goddard Earth Observing System Chemistry-Climate Model (GEOSCCM): LONG-TERM CHANGES IN AGE SPECTRA. Journal of Geophysical Research: Atmospheres, 117(D20). https://doi.org/10.1029/2012JD017905

Liang, Q., Chipperfield, M. P., Fleming, E. L., Abraham, N. L., Braesicke, P., Burkholder, J. B., Daniel, J. S., Dhomse, S., Fraser, P. J., Hardiman, S. C., Jackman, C. H., Kinnison, D. E., Krummel, P. B., Montzka, S. A., Morgenstern, O., McCulloch, A., Mühle, J., Newman, P. A., Orkin, V. L., ... Weiss, R. F. (2017). Deriving Global OH Abundance and Atmospheric 
Lifetimes for Long-Lived Gases: A Search for $\mathrm{CH}_{3} \mathrm{CCl}_{3}$ Alternatives: $\mathrm{CH}_{3} \mathrm{CCl}_{3}$

Alternatives for $\mathrm{OH}$ Estimate. Journal of Geophysical Research: Atmospheres, 122(21),

11,914-11,933. https://doi.org/10.1002/2017JD026926

Linstrom, P. (1997). NIST Chemistry WebBook, NIST Standard Reference Database 69 [Data set]. National Institute of Standards and Technology. https://doi.org/10.18434/T4D303

Liou, K.-N. (2002). An introduction to atmospheric radiation (2nd ed). Academic Press.

Lutgens, F. K., \& Tarbuck, E. J. (2001). The Atmosphere: An Introduction to Meteorology (8th ed). Prentice Hall.

Maasakkers, J. D., Jacob, D. J., Sulprizio, M. P., Scarpelli, T. R., Nesser, H., Sheng, J.-X., Zhang, Y., Hersher, M., Bloom, A. A., Bowman, K. W., Worden, J. R., Janssens-Maenhout, G., \& Parker, R. J. (2019). Global distribution of methane emissions, emission trends, and $\mathrm{OH}$ concentrations and trends inferred from an inversion of GOSAT satellite data for 20102015. Atmospheric Chemistry and Physics, 19(11), 7859-7881. https://doi.org/10.5194/acp-19-7859-2019

Mackay, C., Pandow, M., \& Wolfgang, R. (1963). On the chemistry of natural radiocarbon. Journal of Geophysical Research, 68(13), 3929-3931. https://doi.org/10.1029/JZ2068i013p03929

Montzka, S. A., Krol, M. C., Dlugokencky, E., Hall, B., Jockel, P., \& Lelieveld, J. (2011). Small Interannual Variability of Global Atmospheric Hydroxyl. Science, 331(6013), 67-69. https://doi.org/10.1126/science.1197640 
Montzka, S. A., Spivakovsky, C. M., Butler, J. H., Elkins, J. W., Lock, L. T., \& Mondeel, D. J. (2000). New Observational Constraints for Atmospheric Hydroxyl on Global and Hemispheric Scales. Science, 288(5465), 500-503. https://doi.org/10.1126/science.288.5465.500

Murray, L. T., Mickley, L. J., Kaplan, J. O., Sofen, E. D., Pfeiffer, M., \& Alexander, B. (2014). Factors controlling variability in the oxidative capacity of the troposphere since the Last Glacial Maximum. Atmospheric Chemistry and Physics, 14(7), 3589-3622. https://doi.org/10.5194/acp-14-3589-2014

Naik, V., Voulgarakis, A., Fiore, A. M., Horowitz, L. W., Lamarque, J.-F., Lin, M., Prather, M. J., Young, P. J., Bergmann, D., Cameron-Smith, P. J., Cionni, I., Collins, W. J., Dalsøren, S. B., Doherty, R., Eyring, V., Faluvegi, G., Folberth, G. A., Josse, B., Lee, Y. H., ... Zeng, G. (2013). Preindustrial to present-day changes in tropospheric hydroxyl radical and methane lifetime from the Atmospheric Chemistry and Climate Model Intercomparison Project (ACCMIP). Atmospheric Chemistry and Physics, 13(10), 5277-5298. https://doi.org/10.5194/acp-13-5277-2013

NCEI. (2018, November 5). NUCAPS: NOAA Unique Combined Atmospheric Processing System Environmental Data Record (EDR) Products. NOAA National Centers for Environmental Information. https://data.nodc.noaa.gov/cgi-bin/iso?id=gov.noaa.ncdc:C00868 Network for the Detection of Atmospheric Composition Change. (2021). http://www.ndaccdemo.org/

Pandow, M., Mackay, C., \& Wolfgang, R. (1960). The reaction of atomic carbon with oxygen: Significance for the natural radio-carbon cycle. Journal of Inorganic and Nuclear Chemistry, 14(3-4), 153-158. https://doi.org/10.1016/0022-1902(60)80251-5 
Patra, P. K., Krol, M. C., Montzka, S. A., Arnold, T., Atlas, E. L., Lintner, B. R., Stephens, B. B., Xiang, B., Elkins, J. W., Fraser, P. J., Ghosh, A., Hintsa, E. J., Hurst, D. F., Ishijima, K., Krummel, P. B., Miller, B. R., Miyazaki, K., Moore, F. L., Mühle, J., ... Young, D. (2014). Observational evidence for interhemispheric hydroxyl-radical parity. Nature, 513(7517), 219-223. https://doi.org/10.1038/nature13721

Prather, M. J., Holmes, C. D., \& Hsu, J. (2012). Reactive greenhouse gas scenarios: Systematic exploration of uncertainties and the role of atmospheric chemistry: ATMOSPHERIC CHEMISTRY AND GREENHOUSE GASES. Geophysical Research Letters, 39(9), n/a-n/a. https://doi.org/10.1029/2012GL051440

Prinn, R. G., Huang, J., Weiss, R. F., Cunnold, D. M., Fraser, P. J., Simmonds, P. G., McCulloch, A., Harth, C., Salameh, P., O’Doherty, S., Wang, R. H. J., Porter, L., \& Miller, B. R. (2001). Evidence for Substantial Variations of Atmospheric Hydroxyl Radicals in the Past Two Decades. Science, 292(5523), 1882-1888. https://doi.org/10.1126/science.1058673

Rodgers, C. D. (2000). Inverse Methods for Atmospheric Sounding: Theory and Practice (Vol. 2). WORLD SCIENTIFIC. https://doi.org/10.1142/3171

Rodgers, C. D., \& Connor, B. J. (2003). Intercomparison of remote sounding instruments: INTERCOMPARISON OF REMOTE SOUNDERS. Journal of Geophysical Research: Atmospheres, 108(D3), 4116. https://doi.org/10.1029/2002JD002299

Seidel, D. J., Qiang, F., Randel, W. J., \& Reichler, T. J. (2008). Widening of the tropical belt in a changing climate. Nature Geoscience, 1, 21-24. https://doi.org/10.1038/ngeo.2007.38 
Seidel, D. J., \& Randel, W. J. (2007). Recent widening of the tropical belt: Evidence from tropopause observations. Journal of Geophysical Research, 112(D20), D20113. https://doi.org/10.1029/2007JD008861

Smith, N. (2020, September 15). CO Research data [Personal communication].

Smith, N., \& Barnet, C. D. (2019). Uncertainty Characterization and Propagation in the Community Long-Term Infrared Microwave Combined Atmospheric Product System (CLIMCAPS). Remote Sensing, 11(10), 1227. https://doi.org/10.3390/rs11101227

Smith, N., \& Barnet, C. D. (2020). CLIMCAPS observing capability for temperature, moisture, and trace gases from AIRS/AMSU and CrIS/ATMS. Atmospheric Measurement Techniques, 13(8), 4437-4459. https://doi.org/10.5194/amt-13-4437-2020

Smith, N., Barnet, C. D., \& Esmaili, R. (2020a). CLIMCAPS Uncertainty Application Guide: Working with CLIMCAPS Averaging Kernels. Internal STC, NASA, JPL report. Unpublished.

Smith, N., Barnet, C. D., \& Esmaili, R. (2020b). CLIMCAPS CO application users guide: CLIMCAPS Carbon Monoxide (CO) Retrievals. Internal STC, NASA, JPL report. Unpublished.

SPARC. (2013). SPARC Report on the Lifetimes of Stratospheric Ozone-Deleting Substances, Their Replacements, and Related Species (SPARC Report No. 6; WCRP-15/2013). https://www.sparc-climate.org/publications/sparc-reports/sparc-report-no-6/

Spivakovsky, C. M., Logan, J. A., Montzka, S. A., Balkanski, Y. J., Foreman-Fowler, M., Jones, D. B. A., Horowitz, L. W., Fusco, A. C., Brenninkmeijer, C. A. M., Prather, M. J., Wofsy, S. C., \& McElroy, M. B. (2000). Three-dimensional climatological distribution of tropospheric $\mathrm{OH}$ : Update and evaluation. Journal of Geophysical Research: Atmospheres, 105(D7), 89318980. https://doi.org/10.1029/1999JD901006 
Stone, D., Whalley, L. K., \& Heard, D. E. (2012). Tropospheric OH and HO2 radicals: Field measurements and model comparisons. Chemical Society Reviews, 41(19), 6348. https://doi.org/10.1039/c2cs35140d

Thome, K. (2020, May 19). Terra's Data Record. TERRA: The EOS Flagship; Susannah Pearce and Nate Perrin. https://terra.nasa.gov/terras-data-record

Thompson, A. M., Chappellaz, J. A., Fung, I. Y., \& Kucsera, T. L. (1993). The atmospheric CH4 increase since the Last Glacial Maximum. (2). Interactions with oxidants. Tellus $B, 45(3)$, 242-257. https://doi.org/10.1034/j.1600-0889.1993.t01-2-00003.x

Turner, A. J., Fung, I., Naik, V., Horowitz, L. W., \& Cohen, R. C. (2018). Modulation of hydroxyl variability by ENSO in the absence of external forcing. Proceedings of the National Academy of Sciences, 115(36), 8931-8936. https://doi.org/10.1073/pnas.1807532115

Valin, L. C., Fiore, A. M., Chance, K., \& González Abad, G. (2016). The role of OH production in interpreting the variability of $\mathrm{CH}_{2} \mathrm{O}$ columns in the southeast U.S.: $\mathrm{OH}, \mathrm{VOC}$ and $\mathrm{CH}_{2} \mathrm{O}$ Columns in the SOUTHEAST U.S.A. Journal of Geophysical Research: Atmospheres, 121(1), 478-493. https://doi.org/10.1002/2015JD024012

van Hees, R. M., Tol, P. J. J., Cadot, S., Krijger, M., Persijn, S. T., van Kempen, T. A., Snel, R., Aben, I., \& Hoogeveen, R. W. M. (2018). Determination of the TROPOMI-SWIR instrument spectral response function. Atmospheric Measurement Techniques, 11(7), 3917-3933. https://doi.org/10.5194/amt-11-3917-2018

Voulgarakis, A., Naik, V., Lamarque, J.-F., Shindell, D. T., Young, P. J., Prather, M. J., Wild, O., Field, R. D., Bergmann, D., Cameron-Smith, P., Cionni, I., Collins, W. J., Dalsøren, S. B., Doherty, R. M., Eyring, V., Faluvegi, G., Folberth, G. A., Horowitz, L. W., Josse, B., ... Zeng, 
G. (2013). Analysis of present day and future $\mathrm{OH}$ and methane lifetime in the ACCMIP simulations. Atmospheric Chemistry and Physics, 13(5), 2563-2587.

https://doi.org/10.5194/acp-13-2563-2013

Wang, K.-Y., Shallcross, D. E., \& Pyle, J. A. (2002). Letter to the Editor: Seasonal variations and vertical movement of the tropopause in the UTLS region. Annales Geophysicae, 20(6), 871-874. https://doi.org/10.5194/angeo-20-871-2002

Wang, Y., \& Jacob, D. J. (1998). Anthropogenic forcing on tropospheric ozone and OH since preindustrial times. Journal of Geophysical Research: Atmospheres, 103(D23), 3112331135. https://doi.org/10.1029/1998JD100004

Warner, J. (2020, February 27). NUCAPS Trace Gas Recent Updates. JPSS/GOES-R Proving Ground / Risk Reduction Summit, NOAA Center for Weather and Climate Prediciton Conference Center. https://www.star.nesdis.noaa.gov/star/documents/meetings/2020JPSSGOES/Thursday/ S17_D2_Warner_JPSS_GOESR_PGRR_Summit_202002.pdf

Wolfe, G. M., Nicely, J. M., St. Clair, J. M., Hanisco, T. F., Liao, J., Oman, L. D., Brune, W. B., Miller, D., Thames, A., González Abad, G., Ryerson, T. B., Thompson, C. R., Peischl, J., McKain, K., Sweeney, C., Wennberg, P. O., Kim, M., Crounse, J. D., Hall, S. R., ... DeanDay, J. (2019). Mapping hydroxyl variability throughout the global remote troposphere via synthesis of airborne and satellite formaldehyde observations. Proceedings of the National Academy of Sciences, 116(23), 11171-11180.

https://doi.org/10.1073/pnas.1821661116 
Zhang, L., Jacob, D. J., Liu, X., Logan, J. A., Chance, K., Eldering, A., \& Bojkov, B. R. (2010). Intercomparison methods for satellite measurements of atmospheric composition: Application to tropospheric ozone from TES and OMI. Atmospheric Chemistry and Physics, 10(10), 4725-4739. https://doi.org/10.5194/acp-10-4725-2010

Zhang, X., Jones, D. B. A., Keller, M., Walker, T. W., Jiang, Z., Henze, D. K., Worden, H. M., Bourassa, A. E., Degenstein, D. A., \& Rochon, Y. J. (2019). Quantifying Emissions of CO and NOx Using Observations From MOPITT, OMI, TES, and OSIRIS. Journal of Geophysical Research: Atmospheres, 124(2), 1170-1193.

https://doi.org/10.1029/2018JD028670

Zhang, Y., Jacob, D. J., Maasakkers, J. D., Sulprizio, M. P., Sheng, J.-X., Gautam, R., \& Worden, J. (2018). Monitoring global tropospheric OH concentrations using satellite observations of atmospheric methane. Atmospheric Chemistry and Physics, 18(21), 15959-15973. https://doi.org/10.5194/acp-18-15959-2018

Zhou, M., Langerock, B., Vigouroux, C., Sha, M. K., Hermans, C., Metzger, J.-M., Chen, H., Ramonet, M., Kivi, R., Heikkinen, P., Smale, D., Pollard, D. F., Jones, N., Velazco, V. A., García, O. E., Schneider, M., Palm, M., Warneke, T., \& De Mazière, M. (2019). TCCON and NDACC Xco measurements: Difference, discussion and application. Atmospheric Measurement Techniques, 12(11), 5979-5995. https://doi.org/10.5194/amt-12-59792019

Zhou, M., Langerock, B., Vigouroux, C., Sha, M. K., Ramonet, M., Delmotte, M., Mahieu, E., Bader, W., Hermans, C., Kumps, N., Metzger, J.-M., Duflot, V., Wang, Z., Palm, M., \& De Mazière, M. (2018). Atmospheric $\mathrm{CO}$ and $\mathrm{CH} 4$ time series and seasonal variations on 
Reunion Island from ground-based in situ and FTIR (NDACC and TCCON) measurements. Atmospheric Chemistry and Physics, 18(19), 13881-13901. https://doi.org/10.5194/acp$18-13881-2018$

Ziskin, D. (2019). Measurements Of Pollution In The Troposphere (MOPITT) Level 2 Derived CO (Near and Thermal Infrared Radiances) (MOP02J) V008 Beta [Data set]. NASA Langley Atmospheric Science Data Center DAAC. https://doi.org/10.5067/TERRA/MOPITT/MOP02J_L2.008 


\section{Appendix A}

Appendix A contains additional information referenced in the Literature review. This includes supplemental information on the NDACC network, and the CO retrieval algorithms for MOPITT, CrIS, and TROPOMI.

\section{A.1 Table of NDACC station locations and information}

The table below contains additional information on the NDACC network stations used in this study's analysis. Note that Arrival Heights and Paramaribo stations are not listed here as their datasets did not contain any coinciding CO total column or profile information with this study's satellites. The second Reunion Island station at St. Denis is not included to reduce redundancy. The Harestua station in Norway was not included as the dataset was presented in a seemingly custom format unknown to the authors.

Table 5.2.1: Table of NDACC station locations and information used in this study.

\begin{tabular}{|l|l|l|}
\hline Location & $\begin{array}{l}\text { Station } \\
\text { Representative(s) }\end{array}$ & Station Page \\
\hline $\begin{array}{l}\text { Altzomoni, } \\
\text { Mexico }\end{array}$ & $\begin{array}{l}\text { Dr. Michel Grutter de } \\
\text { la Mora } \\
\text { Centro de Ciencias de } \\
\text { la Atmosfera } \\
\text { Universidad Nacional } \\
\text { Autonoma de Mexico } \\
\text { Mexico }\end{array}$ & $\begin{array}{l}\text { Red Universitaria de Observatorios Atmosfericos (RUOA) } \\
\text { https://www.ruoa.unam.mx }\end{array}$ \\
\hline $\begin{array}{l}\text { Arrival } \\
\text { Heights, } \\
\text { Antarctica }\end{array}$ & $\begin{array}{l}\text { Dan Smale } \\
\text { National Institute of } \\
\text { Water and } \\
\text { Atmospheric Research } \\
\text { (NIWA), Lauder }\end{array}$ & $\begin{array}{l}\text { National Institute of Water and Atmospheric Research } \\
\text { (NIWA) }\end{array}$ \\
$\begin{array}{l}\text { Omakau, Central Otago } \\
\text { New Zealand }\end{array}$ & \\
\hline $\begin{array}{l}\text { Bremen, } \\
\text { Germany }\end{array}$ & $\begin{array}{l}\text { Prof. Dr. Justus Notholt } \\
\text { Institute of } \\
\text { Environmental Physics } \\
\text { University of Bremen }\end{array}$ & $\begin{array}{l}\text { Institute of Environmental Physics } \\
\text { University of Bremen } \\
\text { http://www.iup.uni-bremen.de/ftir/cms/ }\end{array}$ \\
\hline
\end{tabular}




\begin{tabular}{|c|c|c|}
\hline & Bremen, Germany & \\
\hline $\begin{array}{l}\text { Eureka, } \\
\text { Canada }\end{array}$ & $\begin{array}{l}\text { Professor James R. } \\
\text { Drummond } \\
\text { Department of Physics } \\
\text { and Atmospheric } \\
\text { Science } \\
\text { Dalhousie University } \\
\text { Halifax, NS, Canada }\end{array}$ & $\begin{array}{l}\text { Canadian Network for the Detection of Atmospheric } \\
\text { Change (CANDAC) } \\
\text { http://www.candac.ca/candacweb/index.php }\end{array}$ \\
\hline $\begin{array}{l}\text { Izaña, } \\
\text { Tenerife, } \\
\text { Spain }\end{array}$ & $\begin{array}{l}\text { Dr. Emilio Cuevas } \\
\text { Agulló } \\
\text { Izaña Atmospheric } \\
\text { Research Center } \\
\text { AEMET } \\
\text { Spain }\end{array}$ & $\begin{array}{l}\text { Meteorological State Agency of Spain (AEMET) } \\
\text { http://izana.aemet.es/ }\end{array}$ \\
\hline $\begin{array}{l}\text { Jungfraujoch, } \\
\text { Switzerland }\end{array}$ & $\begin{array}{l}\text { Dr. Emmanuel Mahieu } \\
\text { University of Liège } \\
\text { Liège, Belgium }\end{array}$ & $\begin{array}{l}\text { High Altitude Research Stations Jungfraujoch \& Gornergrat } \\
\text { (HFSJG) } \\
\text { http://www.hfsjg.ch/en/jungfraujoch/ }\end{array}$ \\
\hline $\begin{array}{l}\text { Kiruna, } \\
\text { Sweden }\end{array}$ & $\begin{array}{l}\text { Dr. Uwe Raffalski } \\
\text { Institute of Space } \\
\text { Physics } \\
\text { Kiruna, Sweden }\end{array}$ & $\begin{array}{l}\text { Swedish Institute of Space Physics (IRF) } \\
\text { http://www2.irf.se//program/paf/?dbfile=FTIR }\end{array}$ \\
\hline $\begin{array}{l}\text { Lauder, New } \\
\text { Zealand }\end{array}$ & $\begin{array}{l}\text { Richard Querel } \\
\text { National Institute of } \\
\text { Water and } \\
\text { Atmospheric Research } \\
\text { (NIWA), Lauder } \\
\text { Omakau, Central Otago } \\
\text { New Zealand }\end{array}$ & $\begin{array}{l}\text { National Institute of Water and Atmospheric Research } \\
\text { (NIWA) } \\
\text { https://niwa.co.nz/atmosphere/facilities/lauder- } \\
\text { atmospheric-research-station }\end{array}$ \\
\hline $\begin{array}{l}\text { Mauna Loa, } \\
\text { Hawaii, } \\
\text { United States }\end{array}$ & $\begin{array}{l}\text { Dr. Russell C. Schnell } \\
\text { Global Monitoring } \\
\text { Division } \\
\text { NOAA Earth System } \\
\text { Research Laboratory } \\
\text { Colorado, USA }\end{array}$ & $\begin{array}{l}\text { National Oceanic and Atmospheric Administration (NOAA), } \\
\text { Earth System Research Laboratory (ESRL) } \\
\text { https://www.esrl.noaa.gov/gmd/obop/mlo/ }\end{array}$ \\
\hline $\begin{array}{l}\text { Ny Ålesund, } \\
\text { Norway }\end{array}$ & $\begin{array}{l}\text { Prof. Dr. Justus Notholt } \\
\text { Institute of } \\
\text { Environmental Physics } \\
\text { University of Bremen } \\
\text { Bremen, Germany }\end{array}$ & $\begin{array}{l}\text { Natural Environment Research Council, Arctic Office, UK } \\
\text { Arctic Research Station } \\
\text { https://www.arctic.ac.uk/uk-arctic-research-station/ }\end{array}$ \\
\hline $\begin{array}{l}\text { Reunion } \\
\text { Island, } \\
\text { Maido, } \\
\text { France }\end{array}$ & $\begin{array}{l}\text { Dr. V. Duflot } \\
\text { LACy - OSU-R } \\
\text { Reunion Island } \\
\text { University } \\
\text { France }\end{array}$ & $\begin{array}{l}\text { Observatoire de Physique de l'Atmosphère de la Réunion } \\
\text { (OPAR) } \\
\text { https://opar.univ-reunion.fr/ }\end{array}$ \\
\hline $\begin{array}{l}\text { Rikubetsu, } \\
\text { Japan }\end{array}$ & Dr. T. Nagahama & $\begin{array}{l}\text { Geospace Research Center, Solar-Terrestrial Environment } \\
\text { Laboratory, Nagoya University }\end{array}$ \\
\hline
\end{tabular}




\begin{tabular}{|c|c|c|}
\hline & $\begin{array}{l}\text { Solar-Solar Terrestrial } \\
\text { Environment } \\
\text { Laboratory } \\
\text { Nagoya University } \\
\text { Nagoya, Japan }\end{array}$ & $\begin{array}{l}\text { http://center.stelab.nagoya- } \\
\text { u.ac.jp/site1/info e/rikubetsu.html }\end{array}$ \\
\hline $\begin{array}{l}\text { St. } \\
\text { Petersburg, } \\
\text { Russian } \\
\text { Federation }\end{array}$ & $\begin{array}{l}\text { Dr. Maria Makarova } \\
\text { Faculty of Physics } \\
\text { St. Petersburg State } \\
\text { University } \\
\text { St. Petersburg, Russia }\end{array}$ & $\begin{array}{l}\text { Dept. of Atmospheric Physics, Saint-Petersburg State } \\
\text { University } \\
\text { http://troll.phys.spbu.ru/Peterhof FTIR site/welcome.html }\end{array}$ \\
\hline $\begin{array}{l}\text { Thule, } \\
\text { Greenland }\end{array}$ & $\begin{array}{l}\text { Dr. Niels Larsen } \\
\text { Danish Climate Center } \\
\text { Danish Meteorological } \\
\text { Institute } \\
\text { Copenhagen, Denmark }\end{array}$ & $\begin{array}{l}\text { Danish Ministry of the Environment (DMI) } \\
\text { http://ndacc.dmi.dk/ndacc-dmi/ }\end{array}$ \\
\hline $\begin{array}{l}\text { Toronto, } \\
\text { Canada }\end{array}$ & $\begin{array}{l}\text { Dr. Kimberly Strong, } \\
\text { Professor } \\
\text { Department of Physics } \\
\text { University of Toronto } \\
\text { Toronto, Ontario } \\
\text { Canada }\end{array}$ & $\begin{array}{l}\text { University of Toronto Atmospheric Observatory } \\
\text { http://www.atmosp.physics.utoronto.ca/TAO/ }\end{array}$ \\
\hline $\begin{array}{l}\text { Wollongong, } \\
\text { Australia }\end{array}$ & $\begin{array}{l}\text { Dr. Nicholas Jones } \\
\text { Dr. Clare Paton-Walsh } \\
\text { (Murphy) } \\
\text { Prof. David Griffith } \\
\text { Department of } \\
\text { Chemistry } \\
\text { Center for Atmospheric } \\
\text { Chemistry } \\
\text { University of } \\
\text { Wollongong } \\
\text { Australia }\end{array}$ & $\begin{array}{l}\text { University of Wollongong Australia, Centre for Atmospheric } \\
\text { Chemistry } \\
\text { https://www.uow.edu.au/science-medicine- } \\
\text { health/research/centre-for-atmospheric-chemistry/ }\end{array}$ \\
\hline $\begin{array}{l}\text { Zugspitze, } \\
\text { Germany }\end{array}$ & $\begin{array}{l}\text { Dr. Ralf Sussmann } \\
\text { Karlsruhe Institute of } \\
\text { Technology } \\
\text { Institute for } \\
\text { Meteorology and } \\
\text { Climate Research } \\
\text { (IMK-IFU) } \\
\text { Garmisch- } \\
\text { Partenkirchen, } \\
\text { Germany }\end{array}$ & $\begin{array}{l}\text { Karlsruhe Institute of Technology (KIT), Institute of } \\
\text { Meteorology and Climate Research - Atmospheric } \\
\text { Environmental Research } \\
\text { https://www.imk-ifu.kit.edu/311.php }\end{array}$ \\
\hline
\end{tabular}




\section{A.2 MOPITT CO retrieval algorithm flow chart of processing}

The image below summarises the MOPITT CO retrieval algorithm for Level 0 to Level 3 data. In this study, MOPITT Level 2 data is used. Further description can be found in Section 3.1.1.

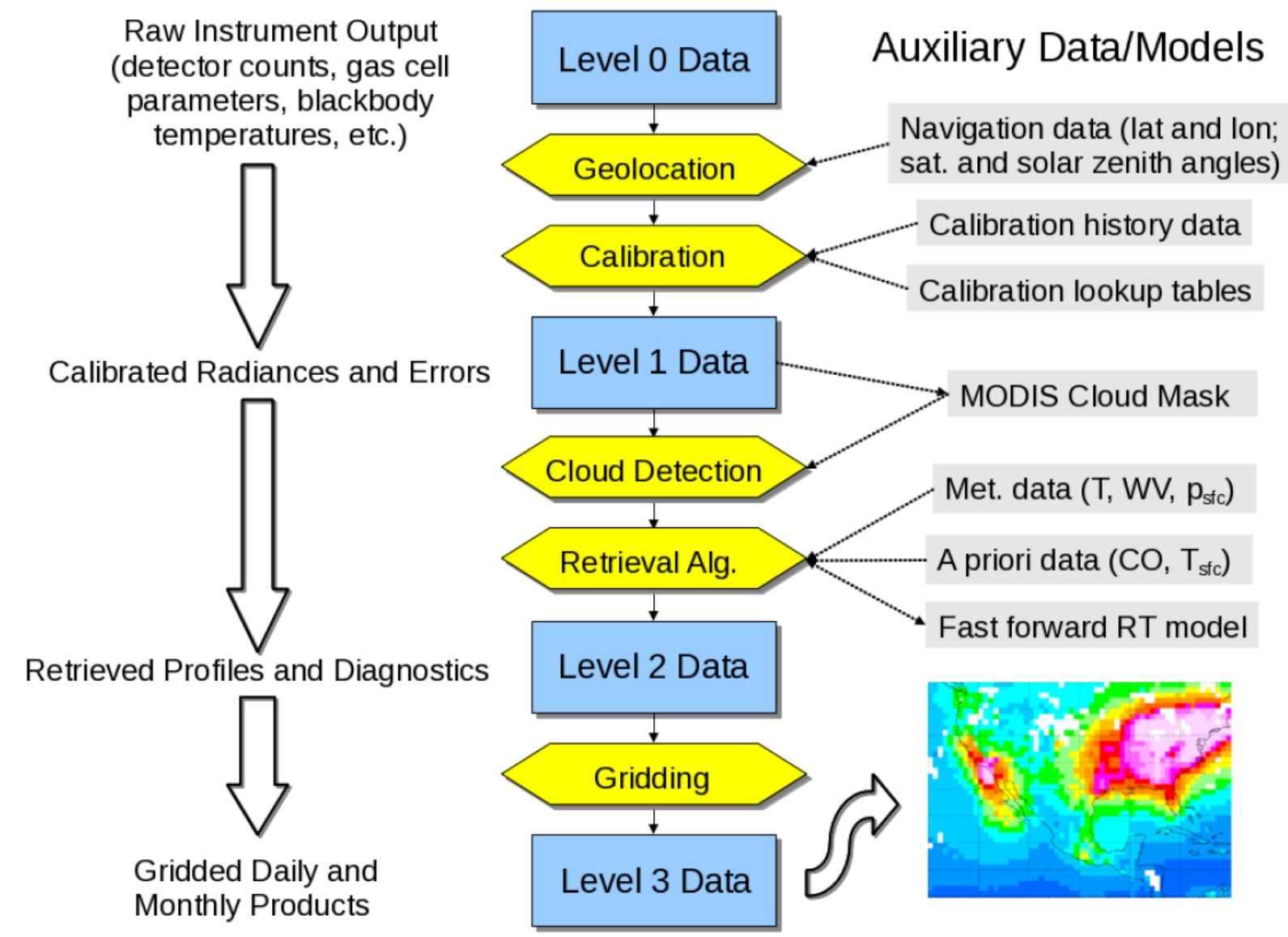

(Francis et al., 2017) 


\section{A.3 CrIS NUCAPS CO retrieval algorithm flow chart}

The image below summarises the CrIS NUCAPS CO retrieval algorithm for Level 1 to

Level 2 data. NUCAPS data is not used in this study but is presented here as it is related to the CLIMCAPS dataset. Further description can be found in Section 3.1.2.

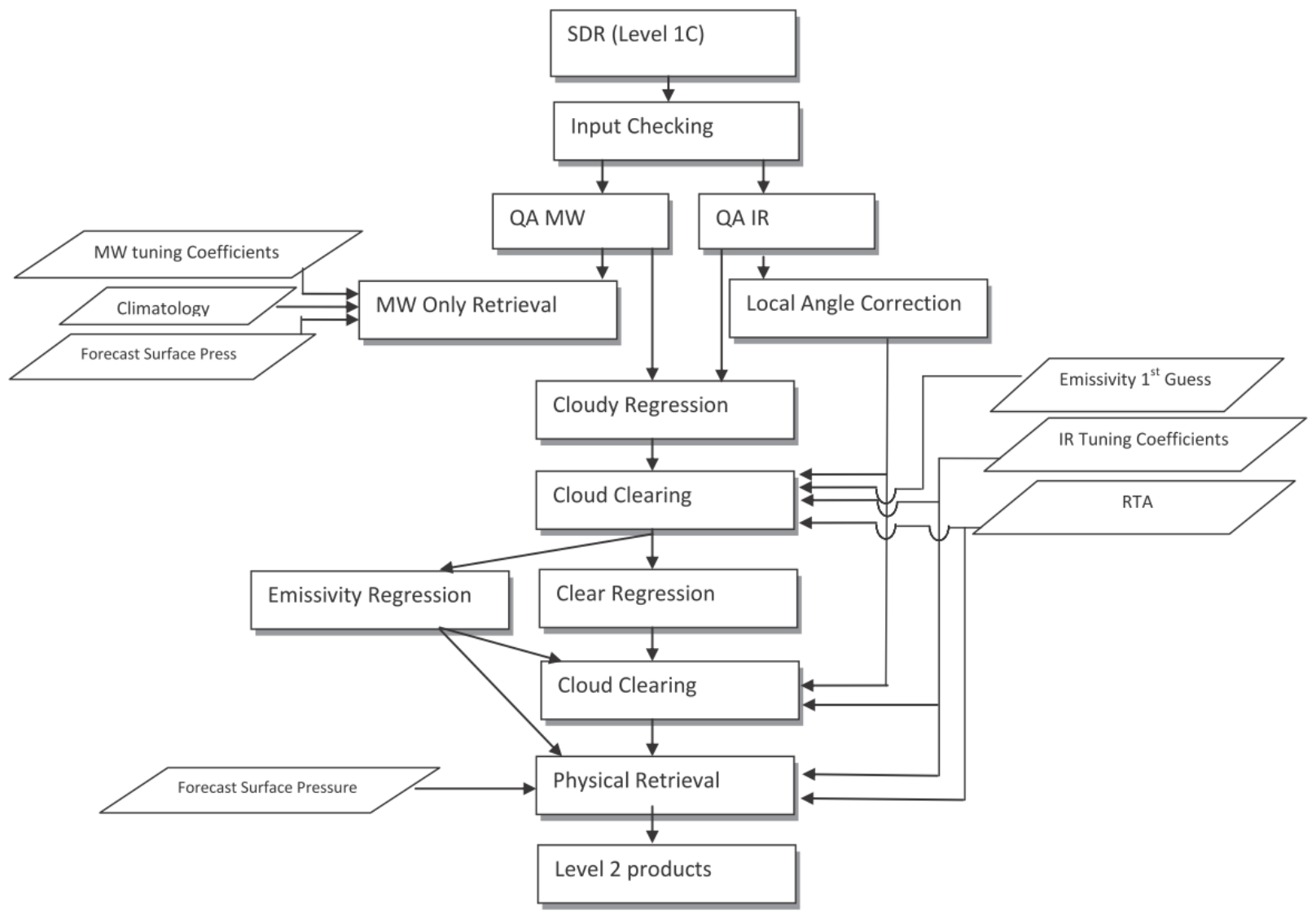

(Gambacorta, 2013)

Table A.3.1: CrIS NUCAPS CO retrieval algorithm for Level 1 to Level 2 data (Gambacorta, 2013) 


\section{Appendix B}

Appendix B contains additional information referenced in the methodology, observation, and discussion sections for this study. This includes supplemental information on missing profile cross-kernel comparisons, additional CO profile error timeseries plots, and additional $\mathrm{CO}$ and $\mathrm{OH}$ distribution plots.

\section{B.1 Cross-kernel RMSE comparisons for high altitude NDACC stations}

Not all data points used in this study have the same surface elevation, including when comparing profile values around each NDACC station. Satellite column and profile data can be taken from a location within range of stations and have a different surface elevation value.

Figure B.1.1 below shows the profile values of the various instruments used over Altzomoni for a specific date when no cross-kernel calculations are applied. Notice that the surface elevation is different for each observing platform is different, with NDACC values ending at $3985 \mathrm{~km}$ (the physical station's location elevation) to the GEOS-Chem values ending at sea level (the elevation from the standard vertical grid).

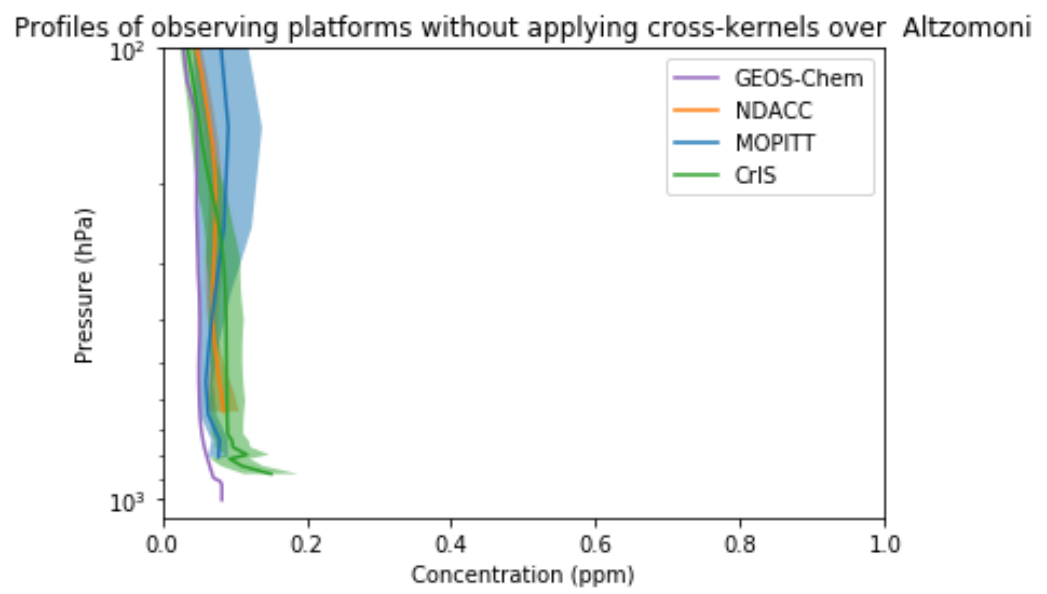

Figure B.1.1: Profile CO concentrations of observing platforms over the NDACC station Altzomoni for July $25^{\text {th }}$ 
Due to these differences in surface elevation values, cross-kernel RMSE calculations are limited to each instrument's respective "cut-off" elevation. For example, the Altzomoni NDACC station is located at an altitude of $3985 \mathrm{~km}$. Therefore, the full profile NDACC cross-kernel RMSE is calculated for all instruments at $3985 \mathrm{~km}$ and above (Figure B.1.2). The MOPITT full profile cross-kernel RMSE calculation incorporates all instruments' values at MOPITT's average surface elevation and above, except NDACC's profile RMSE value is calculated at $3985 \mathrm{~km}$ and above (Figure B.1.3). Finally, The CrIS full profile cross-kernel RMSE is calculated at CrIS's average surface elevation and above only with GEOS-Chem. Where NDACC and MOPITT's full profile RMSE values are calculated at their respective surface elevations and above (Figure B.1.4).

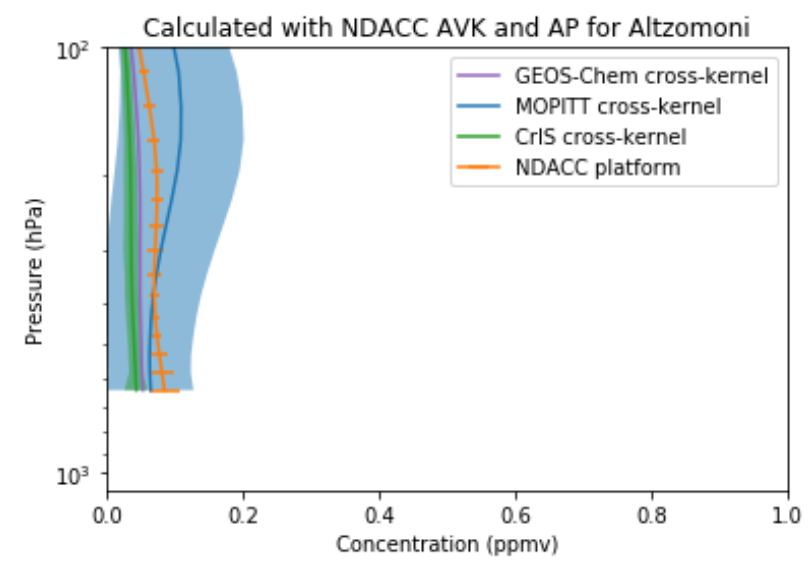

Figure B.1.2: Profile NDACC cross-kernel CO concentrations of observing platforms over the NDACC station Altzomoni for July $25^{\text {th }}$ 


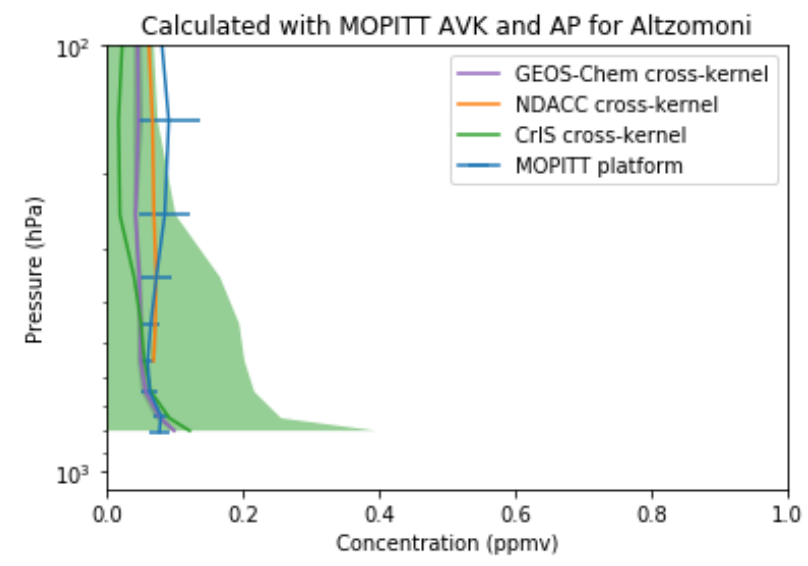

Figure B.1.3: Profile MOPITT cross-kernel CO concentrations of observing platforms over the NDACC station Altzomoni for July $25^{\text {th }}$

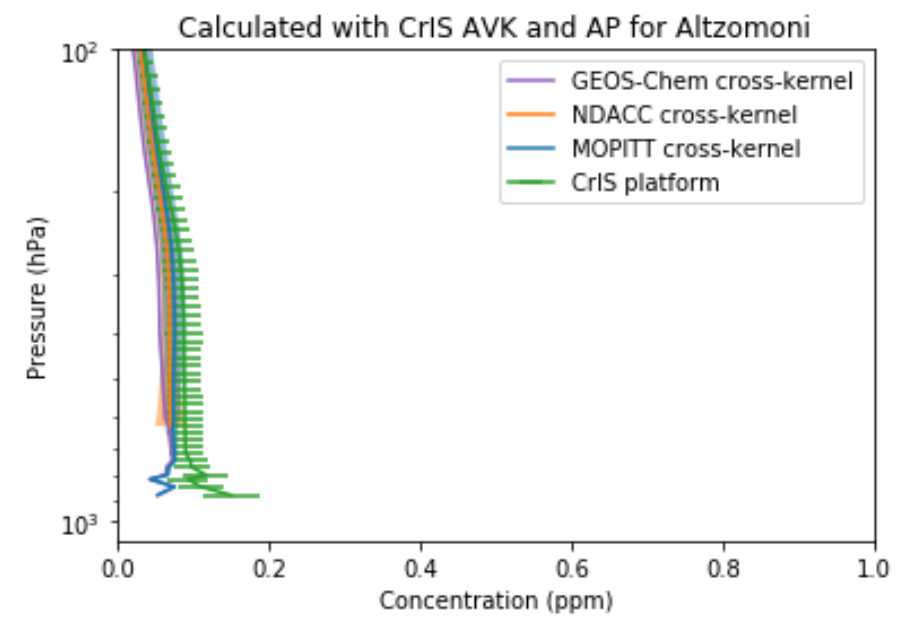

Figure B.1.4: Profile CrIS cross-kernel CO concentrations of observing platforms over the NDACC station Altzomoni for July 25th

Since the Altzomoni NDACC station location located above the boundary layer, not all profile section RMSE values are calculated in Section 4.1.2 as there are no boundary layer observations. Note that there is no BL section RMSE calculated for Altzomoni's MOPITT crosskernel comparison and CrIS cross-kernel comparison with NDACC. The same goes for other comparisons where the NDACC station is located at an elevation above the boundary layer. 


\section{B.2 Additional cross-kernel monthly mean RMSE profile sections}
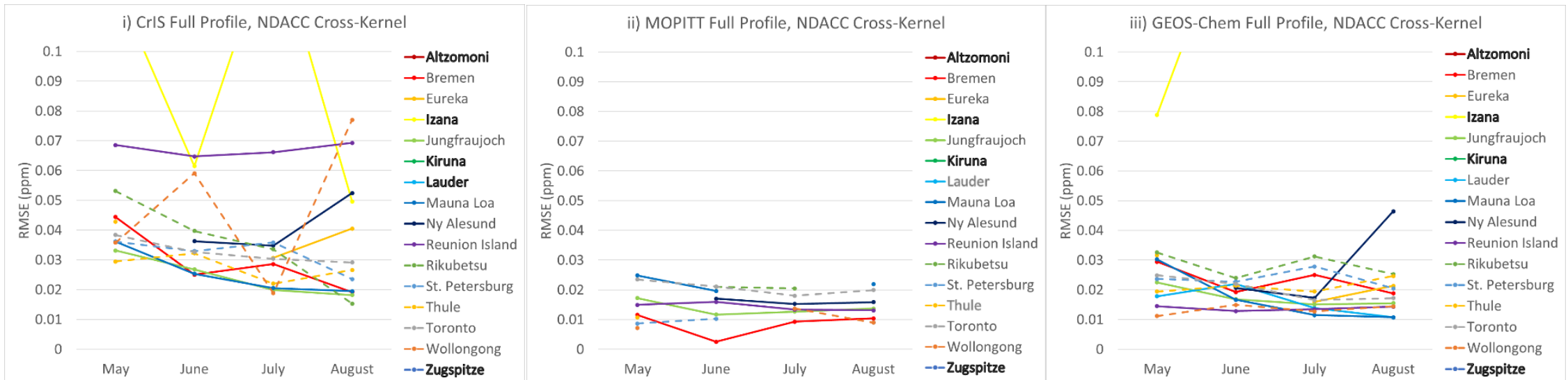

Figure B.2.1: NDACC cross-kernel monthly mean full profile RMSE (ppm) trends against each observing platform. i) CrIS full profile RMSE for NDACC cross-kernel. ii) MOPITT full profile RMSE for NDACC cross-kernel. iii) GEOS-Chem full profile RMSE for NDACC cross-kernel. Stations bolded black in the legend have values off the chart. Stations bolded grey in the legend have no values over the four months.
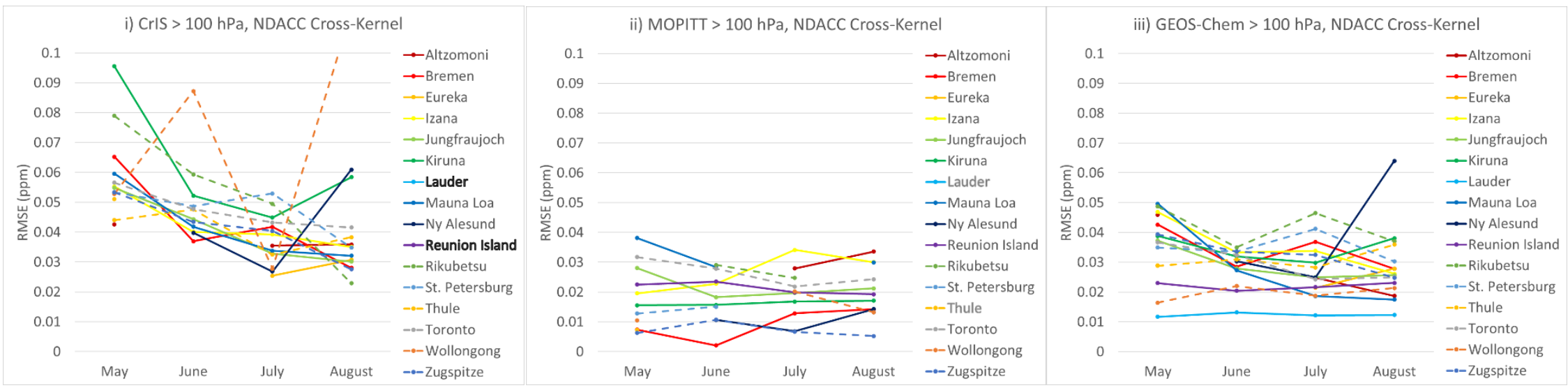

Figure B.2.2: NDACC cross-kernel monthly mean profile RMSE (ppm) trends against each observing platform from 100 hPa to the surface. i) CrIS profile RMSE for NDACC crosskernel from $100 \mathrm{hPa}$ to the surface. ii) MOPITT profile RMSE for NDACC cross-kernel from $100 \mathrm{hPa}$ to the surface. iii) GEOS-Chem profile RMSE for NDACC cross-kernel from 100 hPa to the surface. Stations bolded black in the legend have values off the chart. Stations bolded grey in the legend have no values over the four months. 


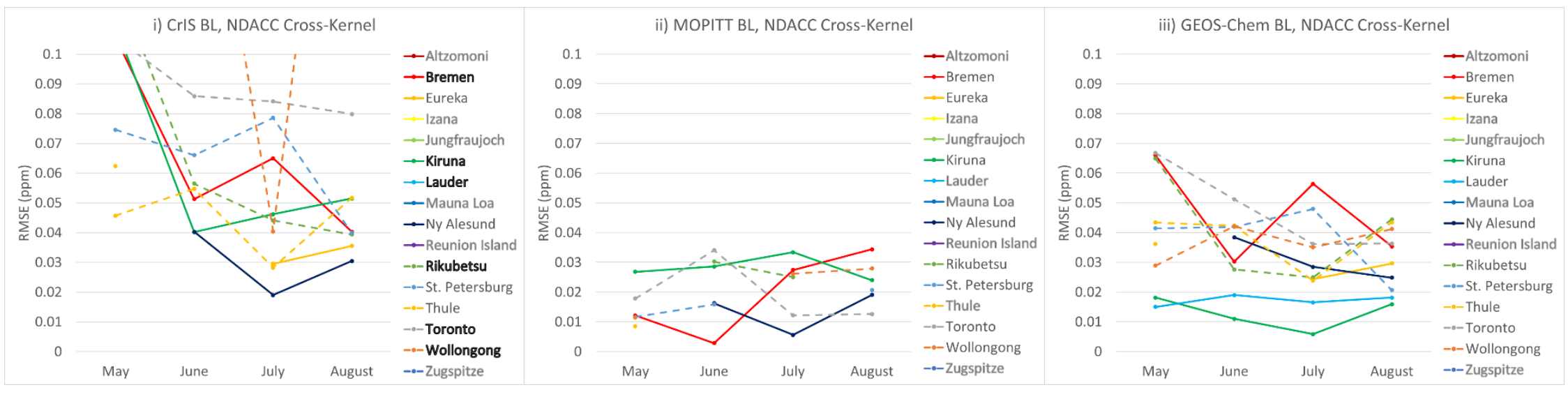

Figure B.2.3: NDACC cross-kernel monthly mean profile RMSE (ppm) trends against each observing platform within the approximate boundary layer (BL) to the surface. i) CrIS profile RMSE for NDACC cross-kernel within the BL to the surface. ii) MOPITT profile RMSE for NDACC cross-kernel within the BL to the surface. iii) GEOS-Chem profile RMSE for NDACC cross-kernel within the BL to the surface. Stations bolded black in the legend have values off the chart. Stations bolded grey in the legend have no values over the four months.

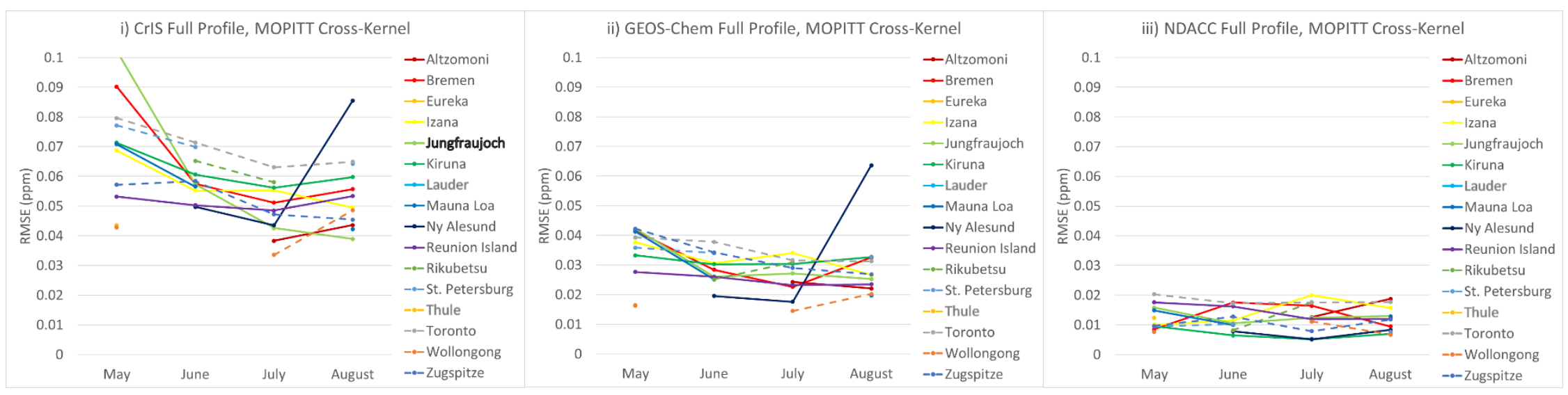

Figure B.2.4: MOPITT cross-kernel monthly mean full profile RMSE (ppm) trends against each observing platform. i) CrIS full profile RMSE for MOPITT cross-kernel. ii) GEOS-Chem full profile RMSE for MOPITT cross-kernel. iii) NDACC full profile RMSE for MOPITT cross-kernel. Note that for MOPITT profiles, the full profile is 100 hPa to the surface. Stations bolded black in the legend have values off the chart. Stations bolded grey in the legend have no values over the four months. 


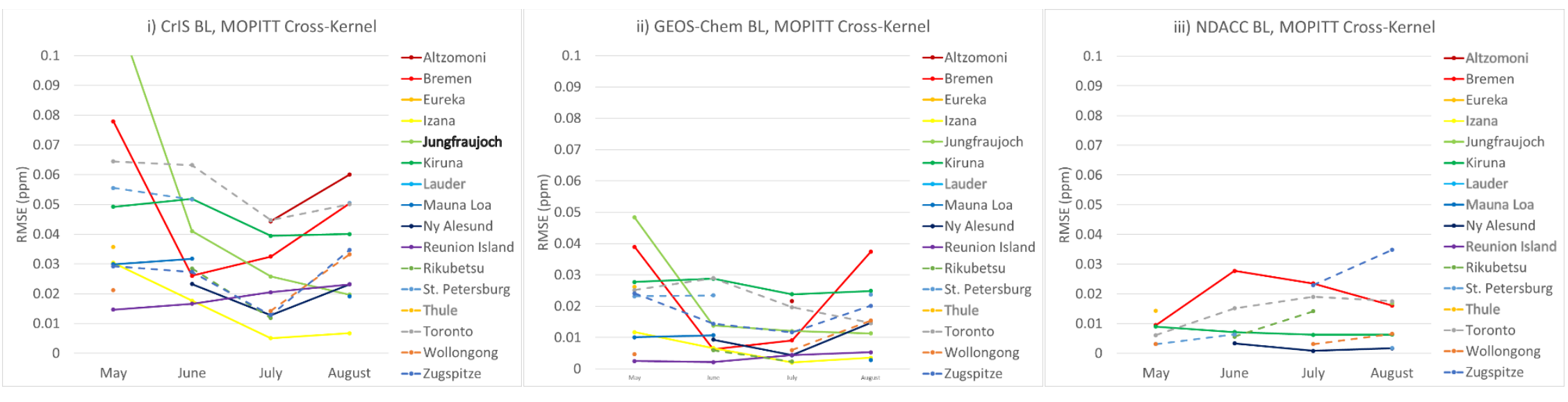

Figure B.2.5: MOPITT cross-kernel monthly mean profile RMSE (ppm) trends against each observing platform within the approximate boundary layer (BL) to the surface. i) CrIS profile RMSE for MOPITT cross-kernel within the BL to the surface. ii) GEOS-Chem profile RMSE for MOPITT cross-kernel within the BL to the surface. iii) NDACC profile RMSE for MOPITT cross-kernel within the BL to the surface. Stations bolded black in the legend have values off the chart. Stations bolded grey in the legend have no values over the four months.

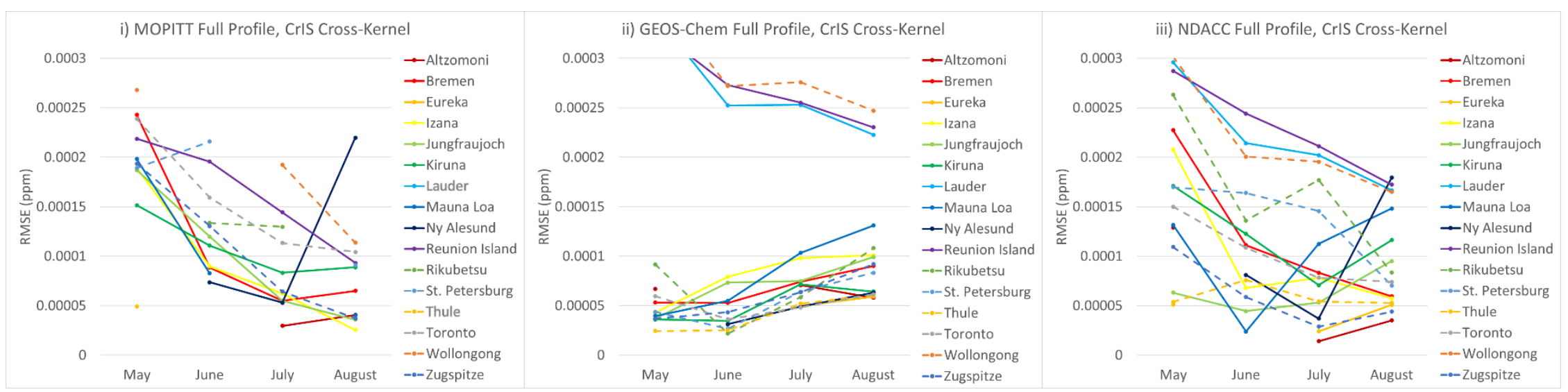

Figure B.2.6: CrIS cross-kernel monthly mean full profile RMSE (ppm) trends against each observing platform. i) MOPITT full profile RMSE for CrIS cross-kernel. ii) GEOS-Chem full profile RMSE for CrIS cross-kernel. iii) NDACC full profile RMSE for CrIS cross-kernel. Stations bolded black in the legend have values off the chart. Stations bolded grey in the legend have no values over the four months. 


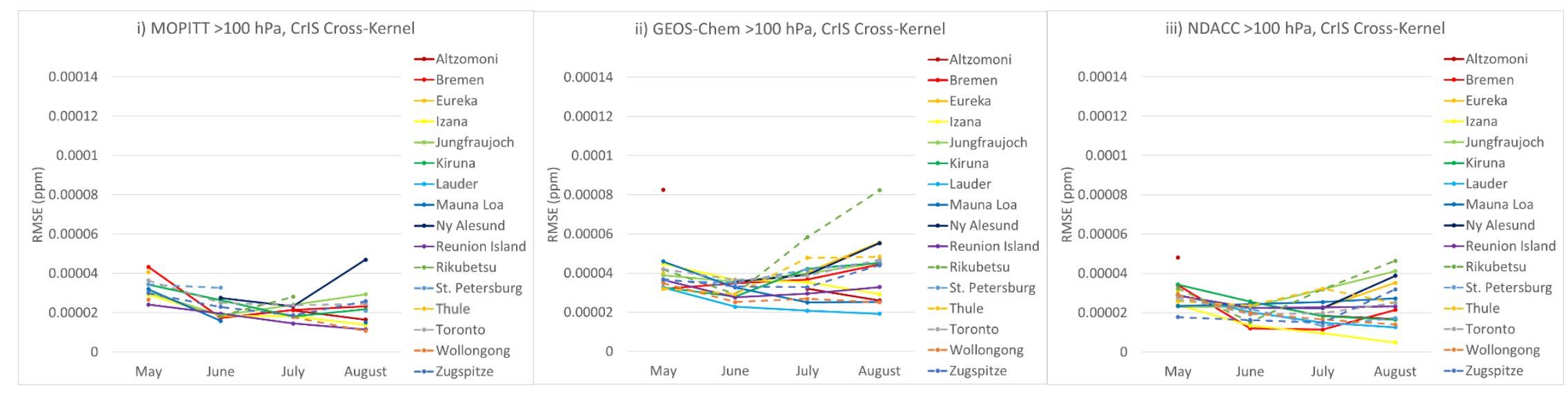

Figure B.2.7: CrIS cross-kernel monthly mean profile RMSE (ppm) trends against each observing platform from 100 hPa to the surface. i) MOPITT profile RMSE for CrIS cross-kernel from $100 \mathrm{hPa}$ to the surface. ii) GEOS-Chem profile RMSE for CrIS cross-kernel from $100 \mathrm{hPa}$ to the surface. iii) NDACC profile RMSE for CrIS cross-kernel from 100 hPa to the surface. Stations bolded black in the legend have values off the chart. Stations bolded grey in the legend have no values over the four months.

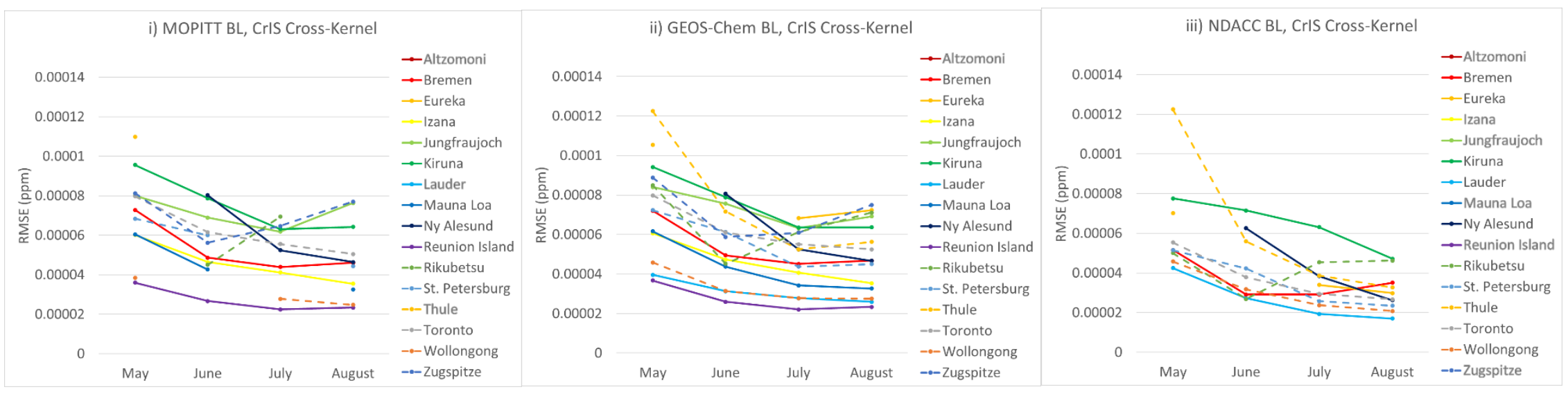

Figure B.2.8: CrIS cross-kernel monthly mean profile RMSE (ppm) trends against each observing platform within the approximate boundary layer (BL) to the surface. i) MOPITT profile RMSE for CrIS cross-kernel within the BL to the surface. ii) GEOS-Chem profile RMSE for CrIS cross-kernel within the BL to the surface. iii) NDACC profile RMSE for CrIS crosskernel within the BL to the surface. Stations bolded black in the legend have values off the chart. Stations bolded grey in the legend have no values over the four months. 


\section{B.3 Additional profile error section graphs}

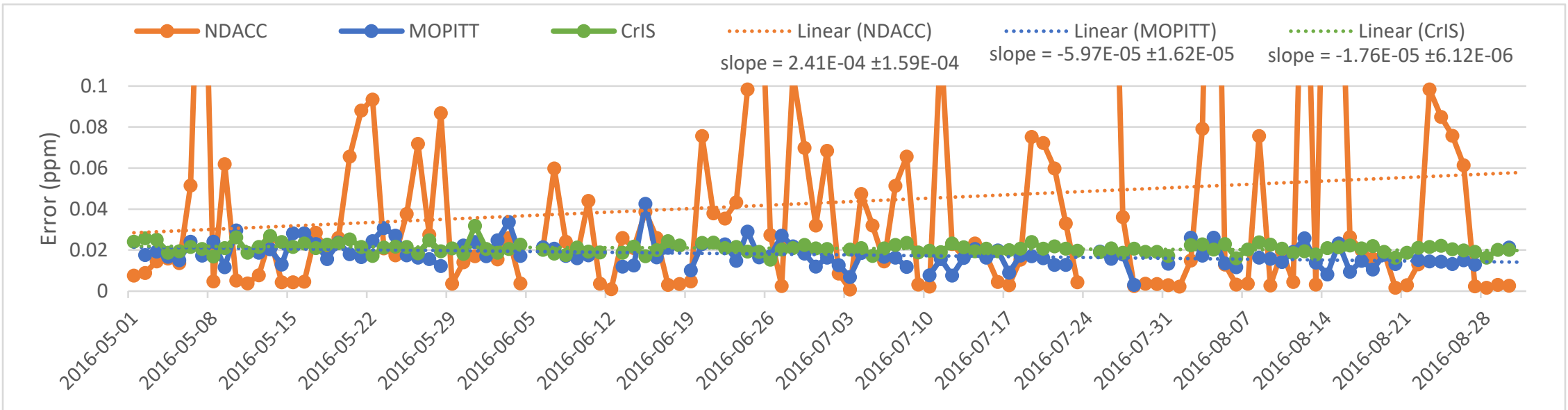

Figure B.3.1: Daily average CO full profile error average for each observing platform for May through August 2016. The profile errors are the provided dataset error values plus the variability of the averaged points.

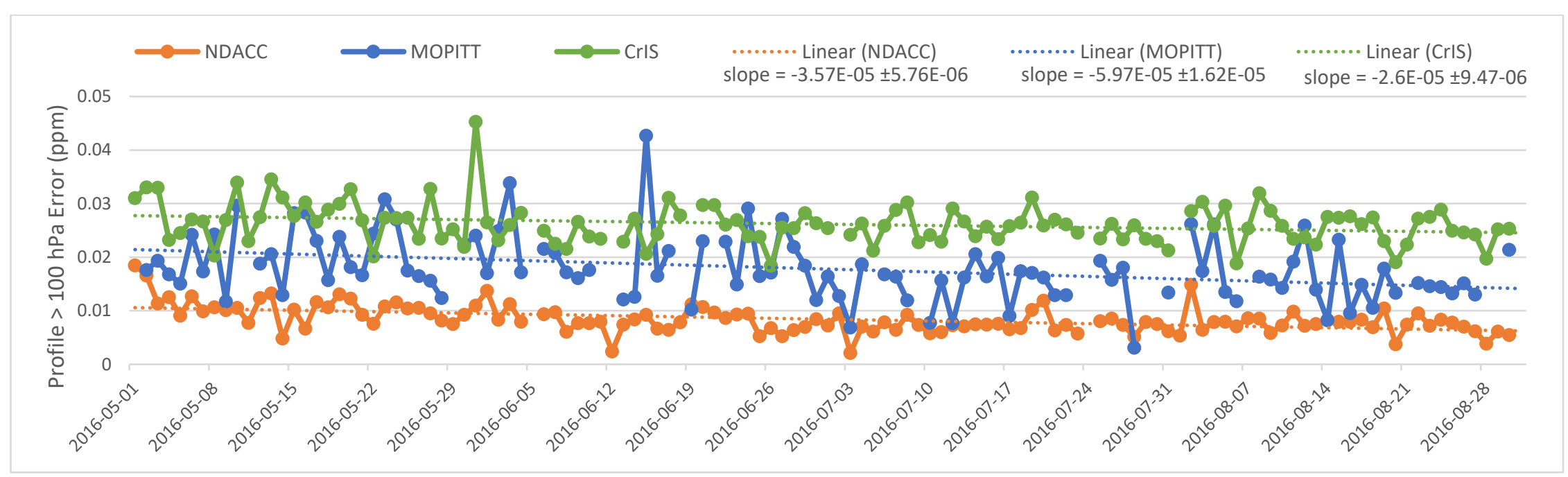

Figure B.3.2: Daily average CO profile error average from the surface to $100 \mathrm{hPa}$ for each observing platform for May through August 2016. The profile errors are the provided dataset error values plus the variability of the averaged points. 


\section{B.4 MOPITT CO distribution}

i) May 2016 MOPITT CO concentrations

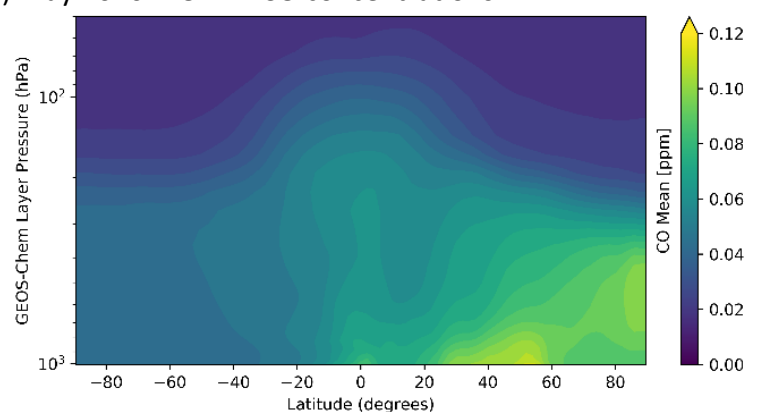

ii) June 2016 MOPITT CO concentrations

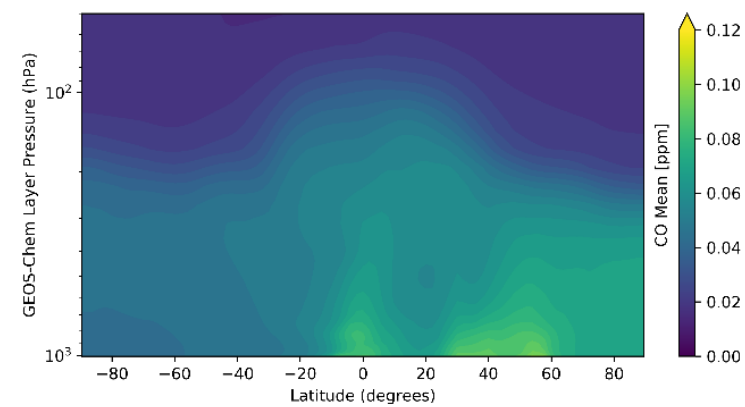

iii) July 2016 MOPITT CO concentrations

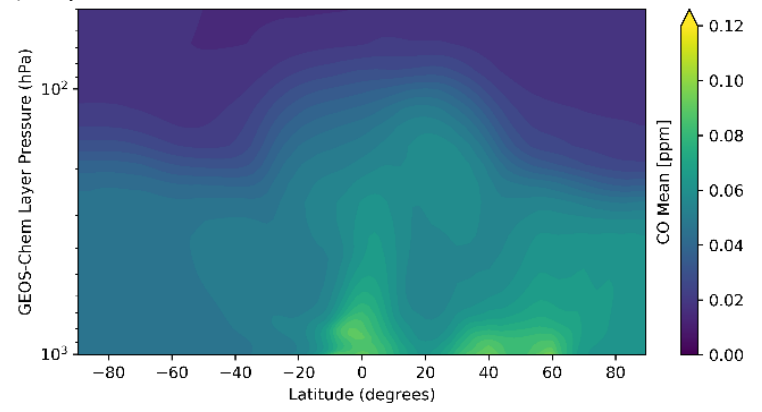

iv) August 2016 MOPITT CO concentrations

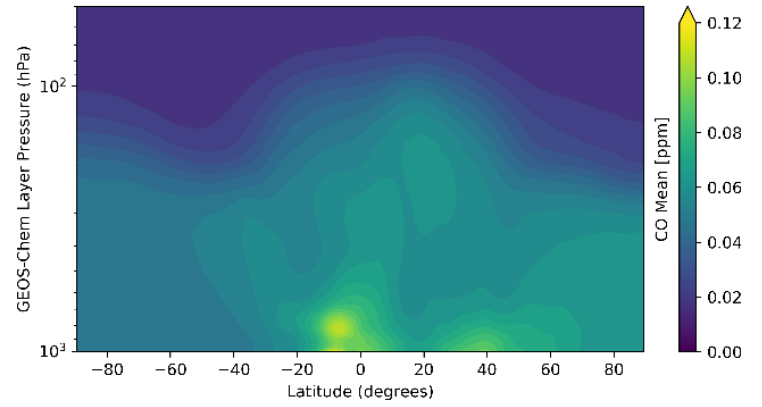

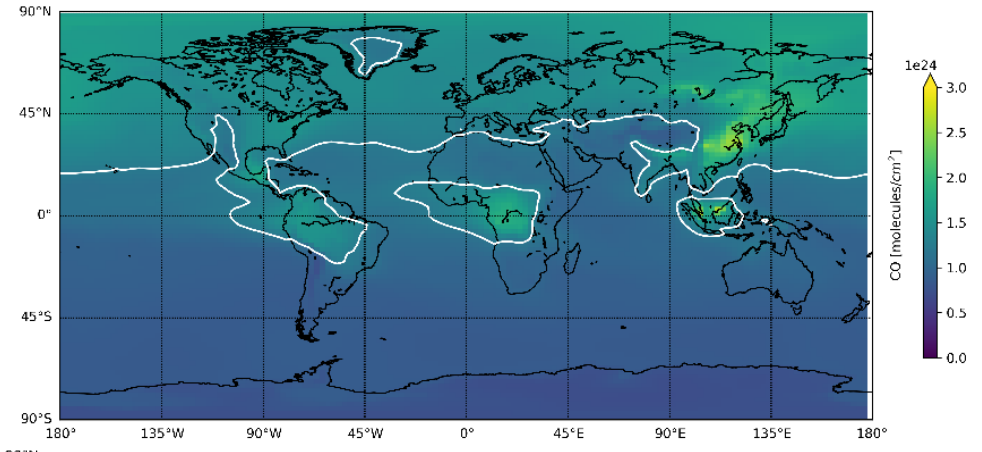
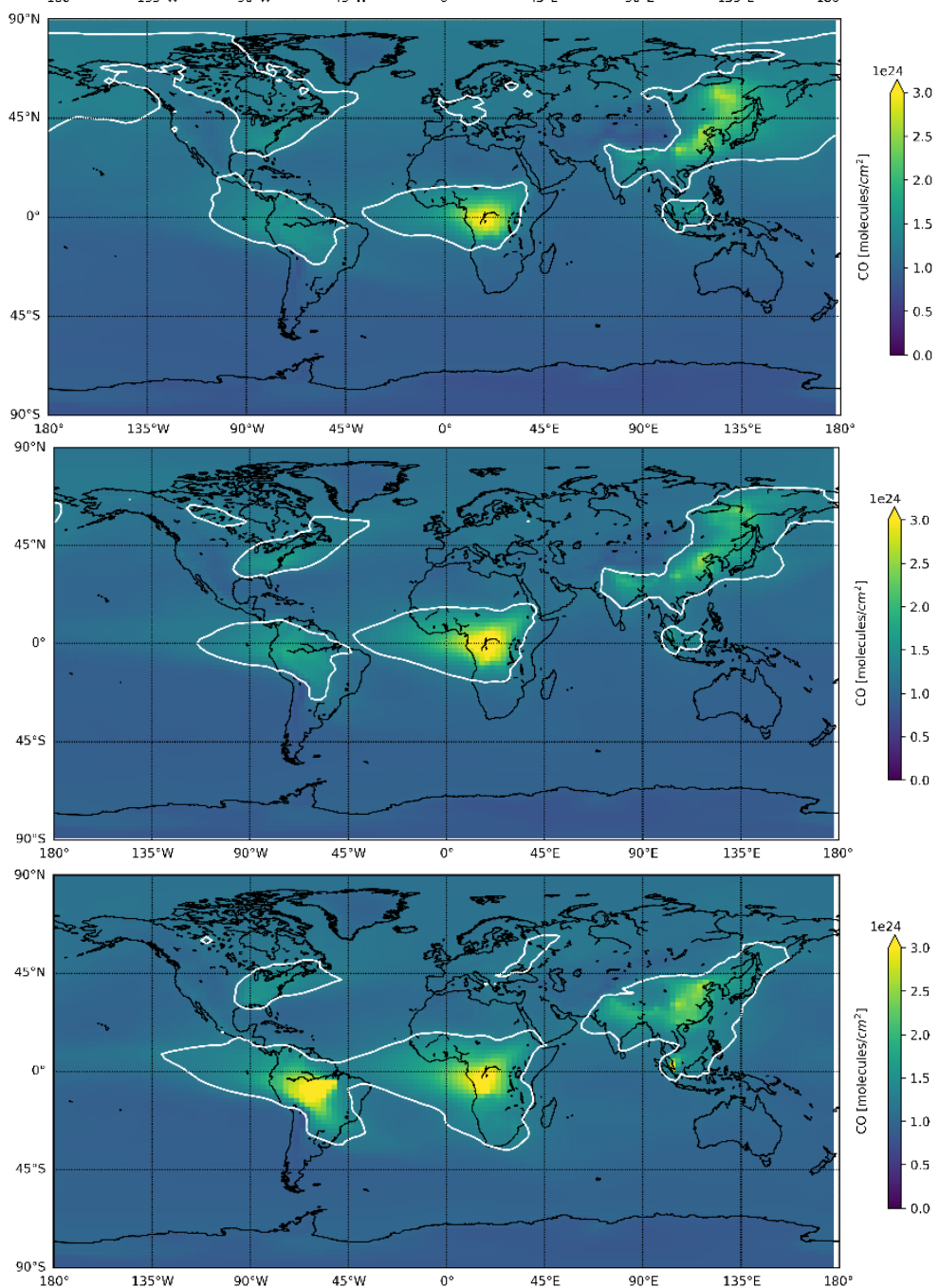

Figure B.4.1: MOPITT modeled mean global CO distribution for each month, May through August 2016. Figures on the left represent the $\mathrm{CO}$ zonal mean ppm across the globe. Figures on the right represent to total column $\mathrm{CO}$ concentration in molecules $/ \mathrm{cm}^{2}$. Note that the MOPITT CO distribution used is the model output after one adjoint iteration. The white contour line represents the MOPITT integrated model global CO concentration's $80^{\text {th }}$ percentile. 


\section{B.5 CO emission sensitivity differences}

Figures B.4.1 to B.4.3 show the MOPITT CO emission sensitivities minus the NDACC sensitivities. Whitespace indicates that sensitivities between the NDACC and MOPITT adjoints are similar. The numerical positive (red) and negative (blue) differences do not particularly reveal much information on sensitivity differences, as their respective sensitivities represent different factors. As mentioned in Section 4.2.1, these figures indicate where MOPITT provides additional or contradictory information against NDACC. Therefore, they only show spatial differences and have no numerical value. These spatial differences in sensitivities appear largest in June and August over land, like the MOPITT CO sensitivity figures.

i) May 2016 MOPITT-NDACC Anthropogenic CO difference

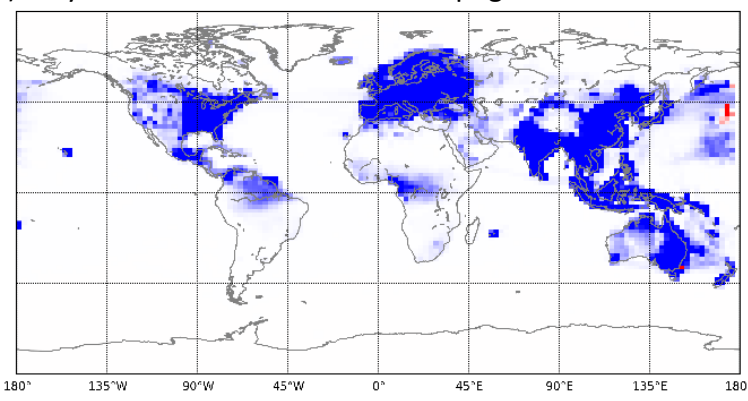

iii) July 2016 MOPITT-NDACC Anthropogenic CO difference

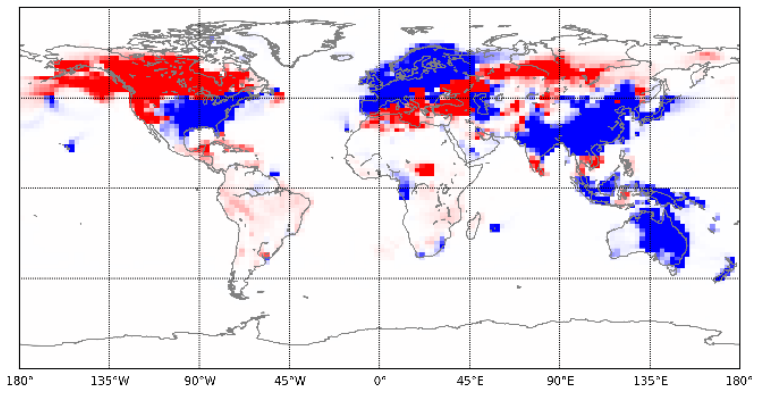

ii) June 2016 MOPITT-NDACC Anthropogenic CO difference

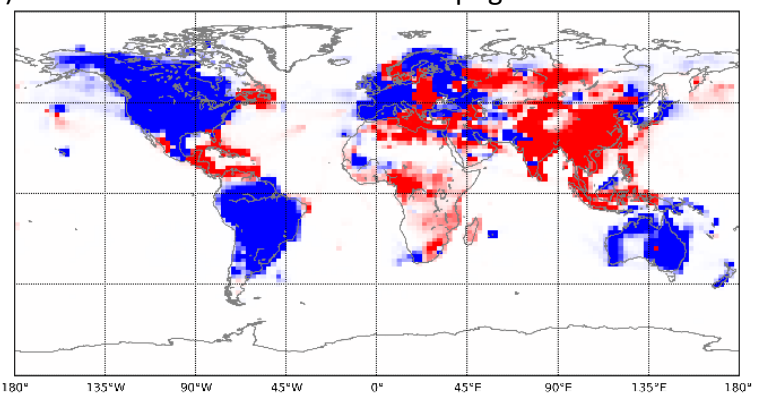

iv) August 2016 MOPITT-NDACC Anthropogenic CO difference

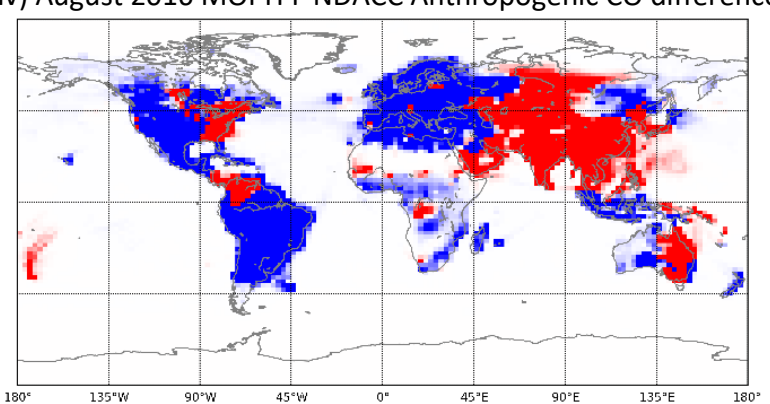

Figure B.5.1: MOPITT model minus GEOS-Chem base surface anthropogenic CO sensitivity [unitless] for each month, May

through August 2016.
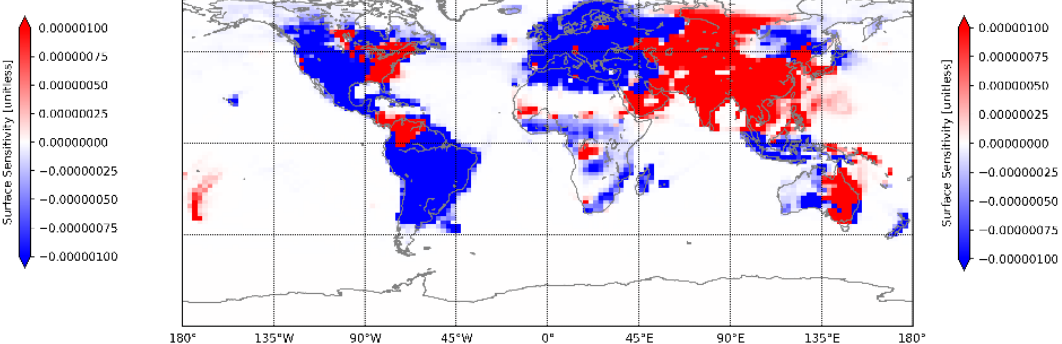
i) May 2016 MOPITT-NDACC Biomass Burning CO difference

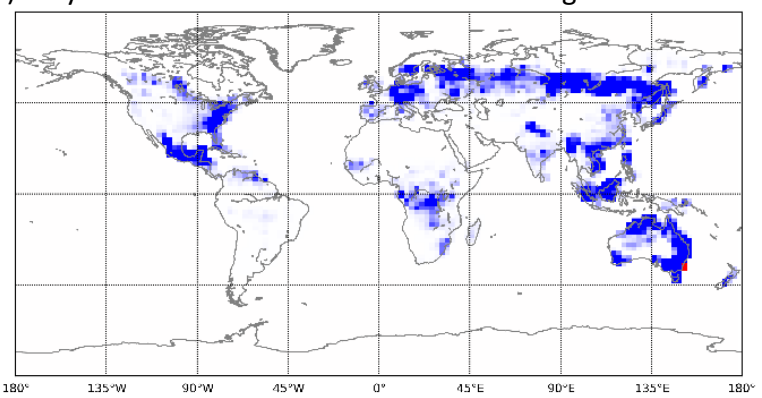

iii) July 2016 MOPITT-NDACC Biomass Burning CO difference

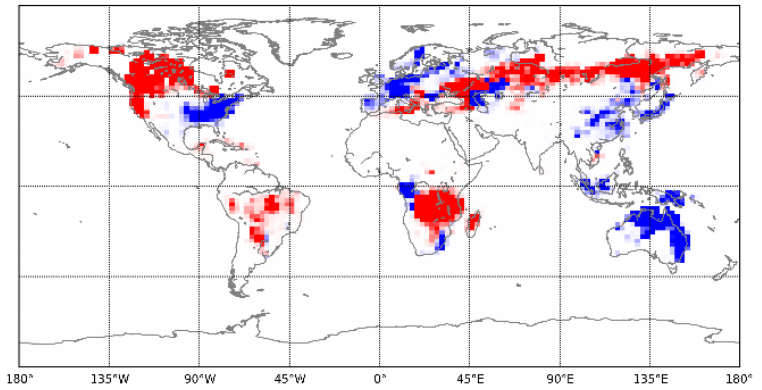

Figure B.5.2: MOPITT model minus GEOS-Chem base surface biomass burning CO sensitivity [unitless] for each month, May through August 2016. ii) June 2016 MOPITT-NDACC Biomass Burning CO difference
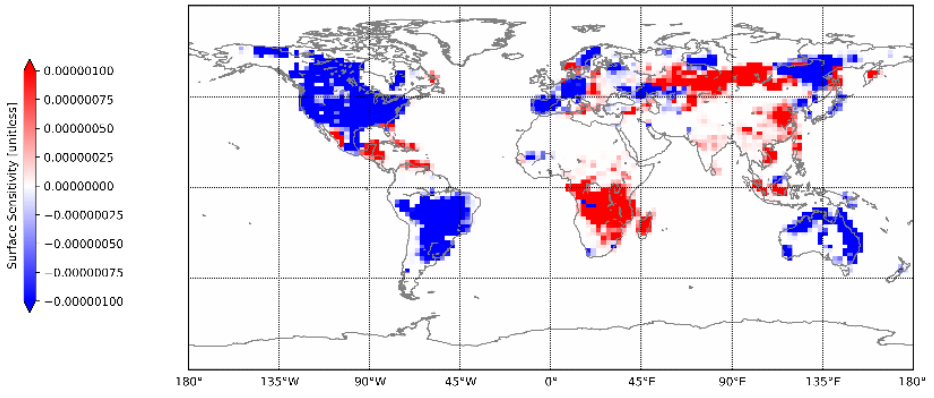

0.000000100
-0.00000075
-0.00000050
-0.000000025
-0.00000000
-0.00000025
0.000000055
-0.00000075
-0.000000100

iv) August 2016 MOPITT-NDACC Biomass Burning CO difference

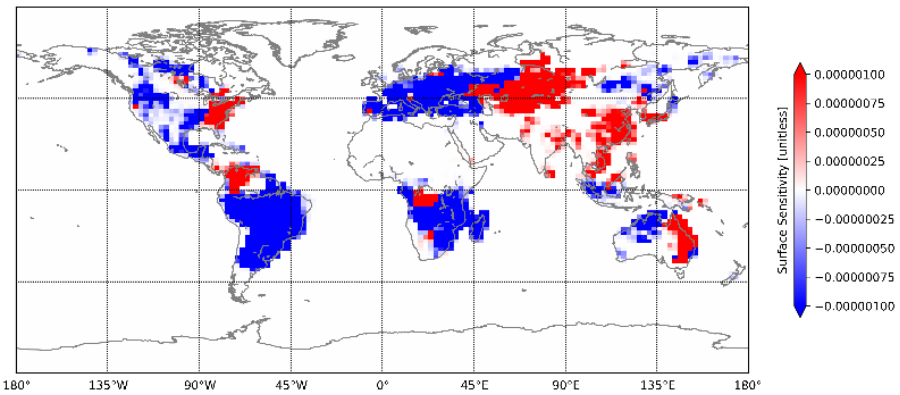

i) May 2016 MOPITT-NDACC Biofuel CO difference

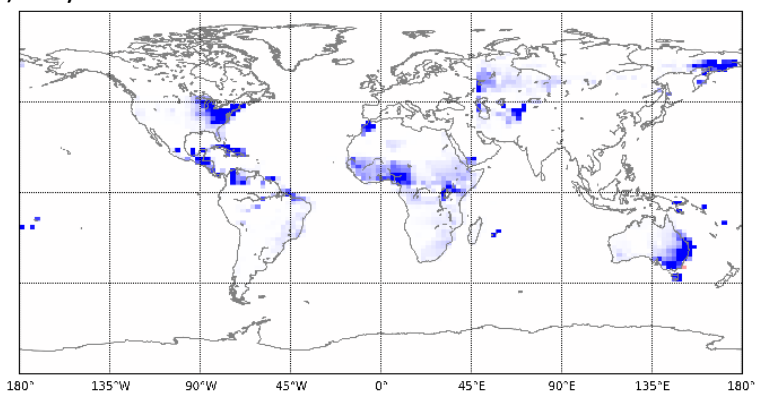

iii) July 2016 MOPITT-NDACC Biofuel CO difference

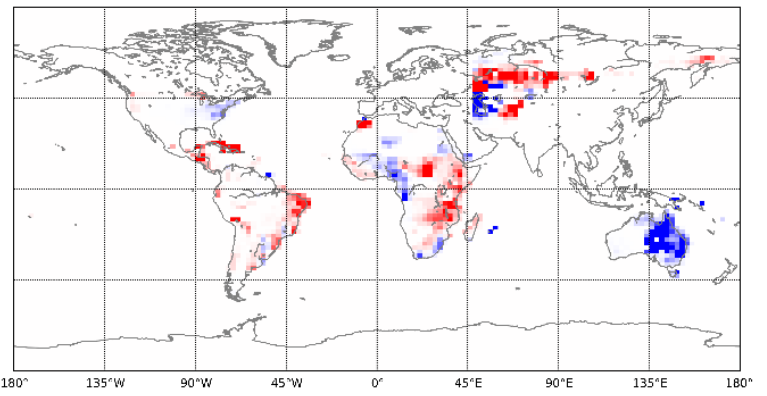

ii) June 2016 MOPITT-NDACC Biofuel CO difference
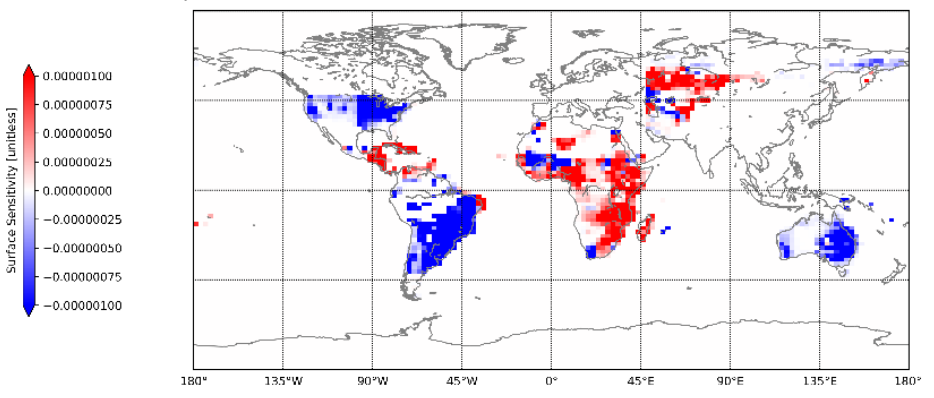

iv) August 2016 MOPITT-NDACC Biofuel CO difference

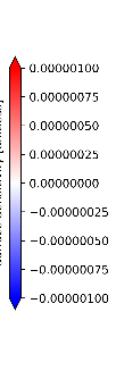

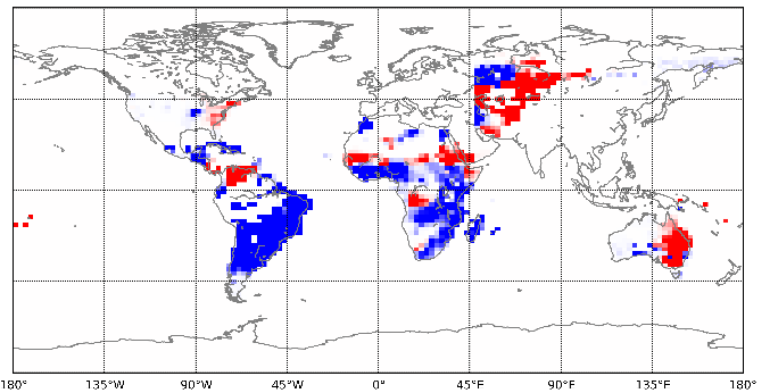

0.00000100 0.00000050 $-0.00000025$ $-0.00000000$ $-0.00000025$ $-0.000000 / 5$ $-0.00000100$

Figure B.5.3: MOPITT model minus GEOS-Chem base surface biofuel CO sensitivity [unitless] for each month, May through August 2016. 


\section{B.6 $\left[\mathrm{CH}_{4}+\mathrm{OH}\right]$ reaction rate sensitivity differences}

Figure B.5.1 shows the MOPITT $\left[\mathrm{CH}_{4}+\mathrm{OH}\right]$ reaction rate sensitivities minus the NDACC sensitivities. Like the $\mathrm{CO}$ emission sensitivity comparisons (Appendix B.4), whitespace indicates that sensitivities between the NDACC and MOPITT adjoints are similar. As mentioned in Section 4.2.2, vertical differences in sensitivity appear to be governed by NDACC reaction sensitivities, with greatest differences occurring over NDACC stations within the Northern Hemisphere between $20^{\circ}$ and $60^{\circ}$ latitude. Sensitivity differences are largest in the Northern Hemisphere, with an approximate separation at the equator appearing similar to the MOPITT sensitivity horizontal distribution (Figure 4.2.2.2). 
i) May 2016 MOPITT-NDACC $\left[\mathrm{CH}_{4}+\mathrm{OH}\right]$ difference

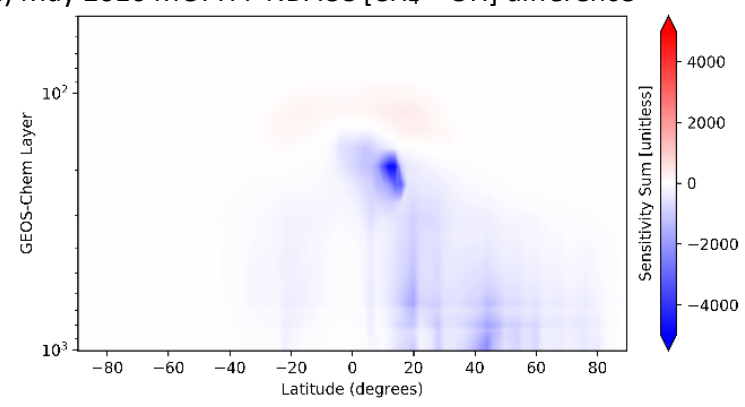

ii) June $2016 \mathrm{MOPITT}-\mathrm{NDACC}\left[\mathrm{CH}_{4}+\mathrm{OH}\right]$ difference

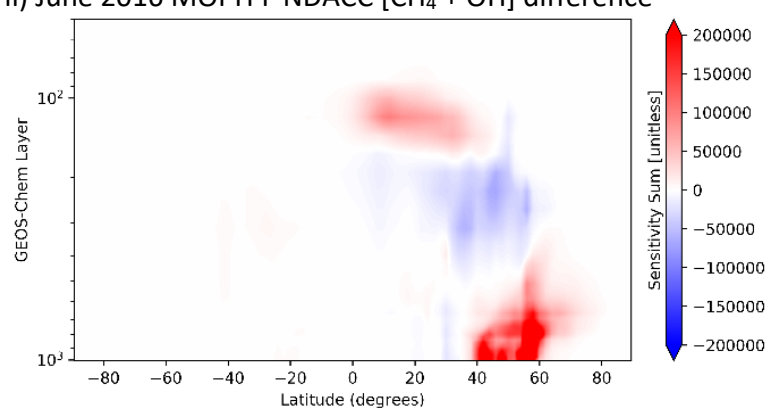

iii) July 2016 MOPITT-NDACC $\left[\mathrm{CH}_{4}+\mathrm{OH}\right]$ difference

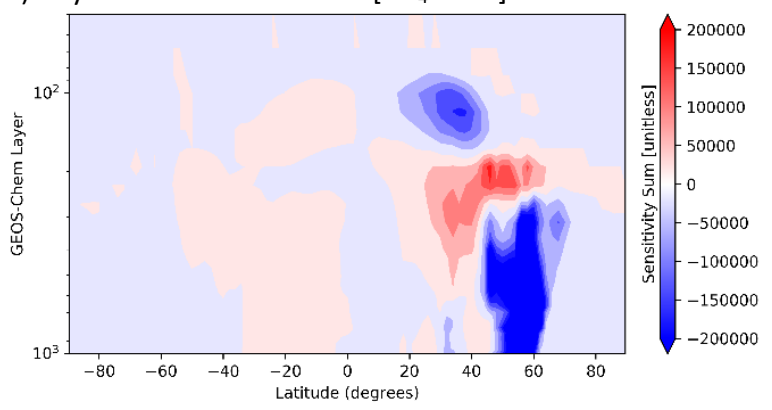

iv) August $2016 \mathrm{MOPITT}-\mathrm{NDACC}\left[\mathrm{CH}_{4}+\mathrm{OH}\right]$ difference

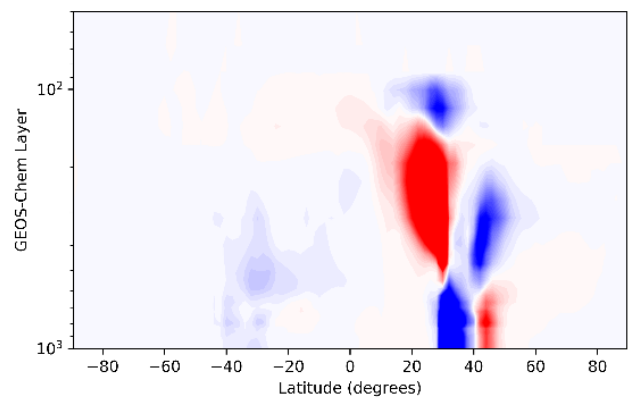

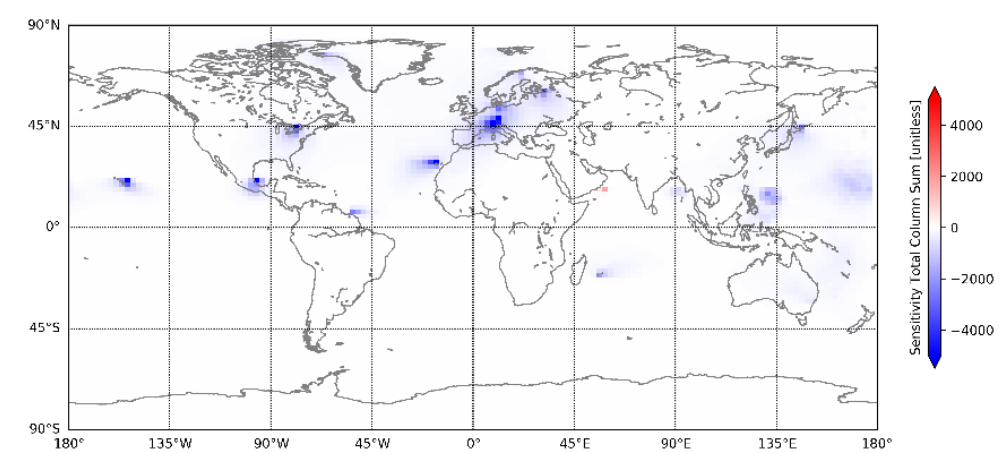
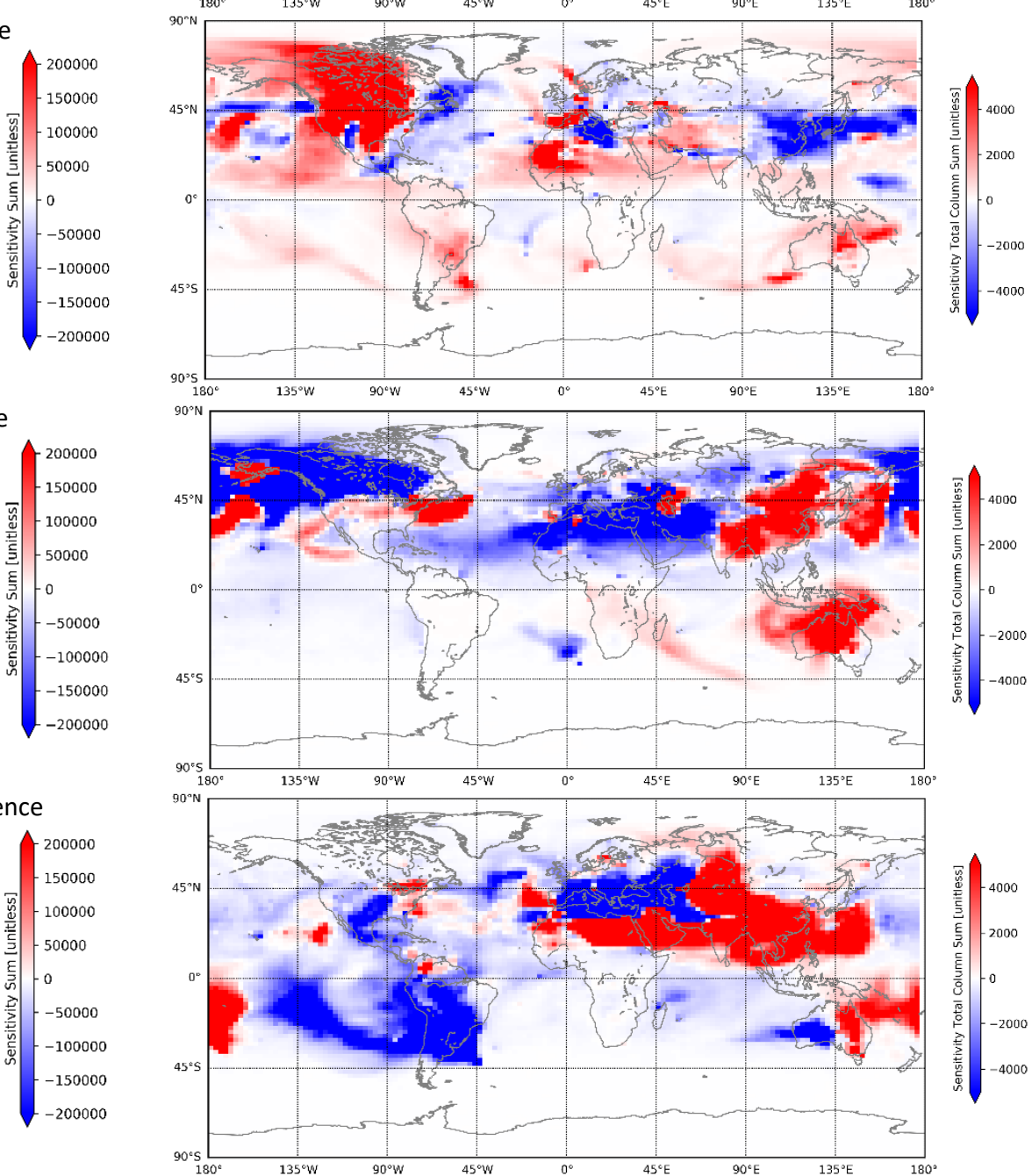

Figure B.6.1: MOPITT minus NDACC global $\left[\mathrm{CH}_{4}+\mathrm{OH}\right]$ reaction sensitivity [unitless] for each month, May through August 2016. Note that the figure scales on the left is different in May than June through August. 


\section{B.7 MOPITT OH Distribution}

i) May $2016 \mathrm{MOPITT}$ global OH

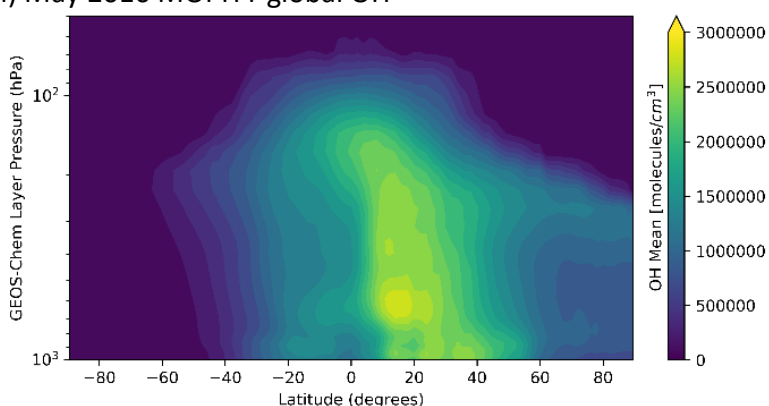

ii) June 2016 MOPITT global OH

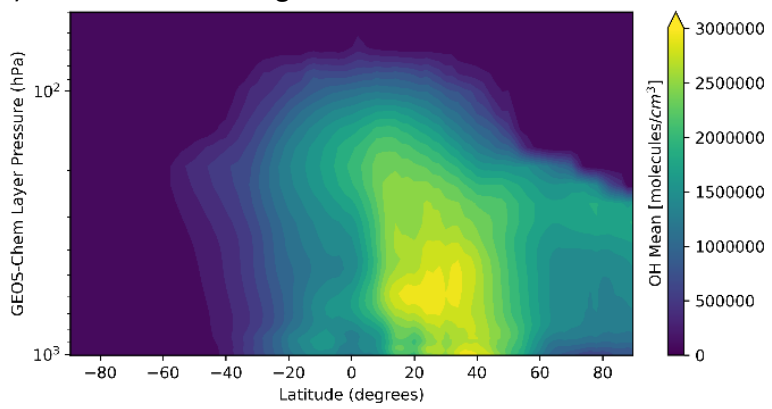

iii) July 2016 MOPITT global OH

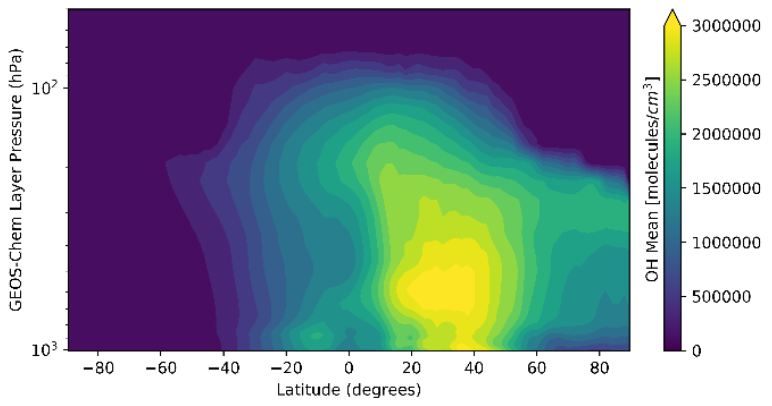

iv) August 2016 MOPITT global OH

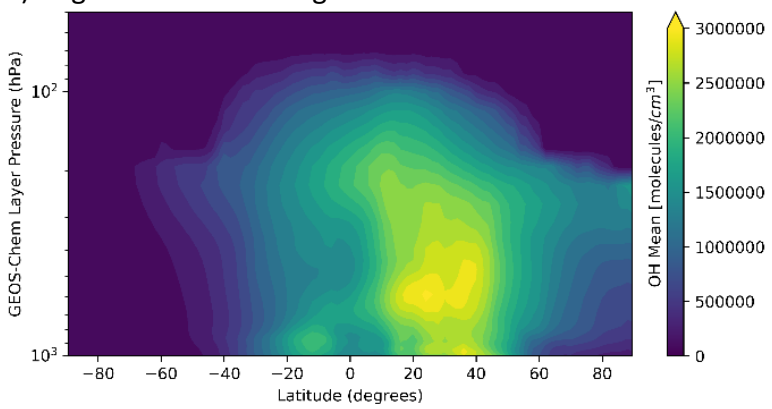

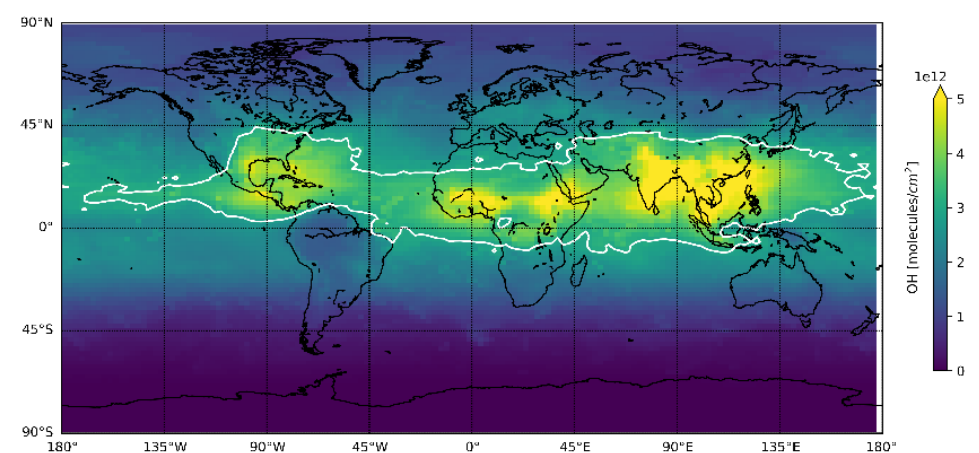
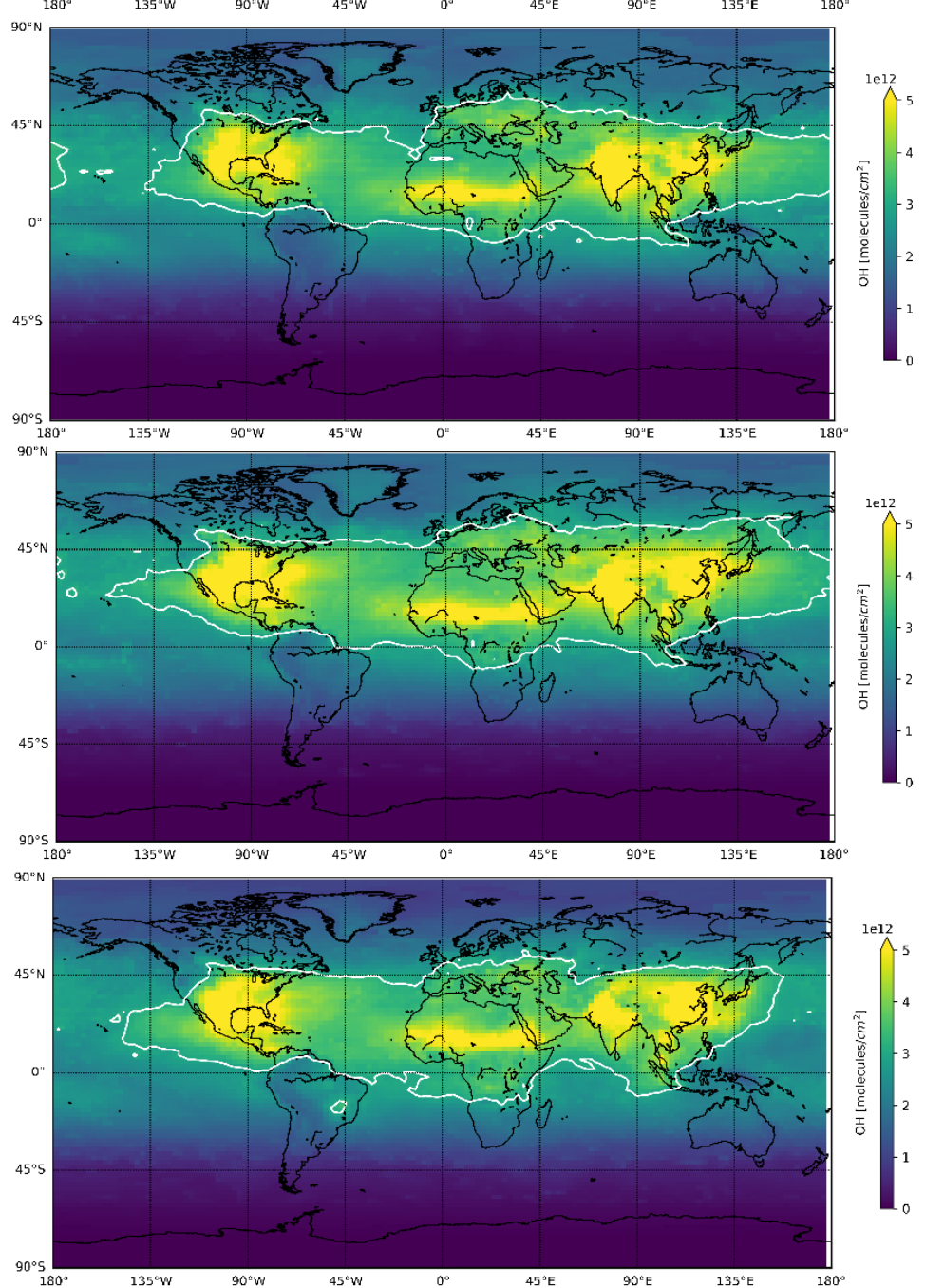

Figure B.7.1: MOPITT base modeled mean global OH distribution for each month, May through August 2016. Figures on the left represent the $\mathrm{OH}$ zonal mean molecules $/ \mathrm{cm}^{3}$ across the globe. Figures on the right represent to total column CO concentration in molecules $/ \mathrm{cm}^{2}$. The white contour line represents the MOPITT integrated model global OH concentration's $80^{\text {th }}$ percentile. 